\title{
Zinc-catalyzed Phosphonylation of Alcohols with Alkyl Phosphites
}

Yuki Saito, Soo Min Cho, Luca Alessandro Danieli, and Shū Kobayashi*

shu_kobayashi@chem.s.u-tokyo.ac.jp

Department of Chemistry, Graduate School of Science, The University of Tokyo, 7-3-1 Hongo, Bunkyo-ku, Tokyo 113-0033, Japan

\section{Supporting Information}

\section{Contents}

1. General

2. A typical procedure of optimization of reaction conditions using cyclohexanol 3a

3. A general procedure of substrate scope with $\mathrm{Zn}(\mathrm{acac})_{2}$

4. A large scale procedure of phosphonylation of alcohol 2a

5. A general procedure of substrate scope with $\mathrm{Zn}(\mathrm{TMHD})_{2}$

6. A procedure of oxidation of $3 \mathrm{~g}$ to $4 \mathrm{~g}$

7. Compound data

8. References 


\section{General}

- JEOL JNM-ECA 500 or ECX 600 spectrometers were used for NMR measurement using deuterated chloroform as solvent unless otherwise noted. Tetramethyl silane was used as an internal standard for 1H-NMR ( $\delta=0 \mathrm{ppm}$ ), and deuterated chloroform was used for 13C$\operatorname{NMR}(\delta=77.36 \mathrm{ppm})$.

- Electrospray ionization (ESI) mass spectra and Direct Analysis in Real Time (DART) mass spectra were recorded on JEOL JMS-T100TD mass spectrometer.

- IR spectra were recorded on Shimadzu IRSpirit-T with QART-S.

- Melting point was recorded with Yazawa Micro Melting Point BY-1.

- Solvents were purchased in anhydrous grade from FUJIFILM Wako Pure Chemical Company and Tokyo Chemical Industry Co., Ltd.. and used as received.

- Metal salts employed for the screening were purchased from Wako Pure Chemical Company and Tokyo Chemical Industry Co., Ltd.. and used as received.

- Alcohols 1a-1p and 1x-1y were purchased from FUJIFILM Wako Pure Chemical Company and Tokyo Chemical Industry Co., Ltd.. and purified by recrystallization or distillation before use.

- Alcohols 1q-1w derived from carbohydrates were synthesized by reported procedure. ${ }^{1-4}$

- Alcohol $1 \mathrm{z}$ derived from serine was synthesized by reported procedure. ${ }^{5}$

- Dimethyl phosphite was purchased from Tokyo Chemical Industry Co., Ltd. and distilled before use.

- Powdered molecular sieves were purchased from Sigma-Aldrich Co. LLC., and dried $200{ }^{\circ} \mathrm{C}$ under vacuo before use and kept in a glove box.

- All commercially-available reagents, unless otherwise stated, were used without further purification.

- All reactions, unless otherwise stated, were carried out under argon atmosphere. 
2. A typical procedure of optimization of reaction conditions using cyclohexanol 1a (Table 1 , entry 6)

To an oven dried test tube equipped with a magnetic stirring bar was added $\mathrm{Zn}$ (acac) $)_{2}$ (7.9 mg, $0.03 \mathrm{mmol})$ and powdered MS 4A (100 mg). The tube was capped with a septa, connected to a vacuum line, then an Ar balloon was equipped. Toluene $(1.0 \mathrm{~mL})$ was added to the tube, followed by the addition of dimethyl phosphite $2(99.0 \mathrm{mg}, 0.9 \mathrm{mmol})$ and cyclohexanol $1 \mathrm{a}(30.0 \mathrm{mg}, 0.3 \mathrm{mmol})$ by syringe. The tube was put in an oil bath heated at $60{ }^{\circ} \mathrm{C}$, and stirred for $18 \mathrm{~h}$. Then, the reaction mixture was cooled to room temperature and diluted with ethyl acetate $(5 \mathrm{~mL})$. The solid material was removed by filtration and washed with ethyl acetate $\left(5 \mathrm{~mL}^{*} 2\right)$. The combined organic phase was evaporated and connected to a vacuum line. The ${ }^{1} \mathrm{H}$ NMR was recorded with 1,2,4,5tetramethyl benzene as internal standard. The crude mixture was purified by column chromatography using hexane/ethyl acetate $=1 / 2$ as eluent $(\mathrm{Rf}=0.3)$. The product $3 \mathrm{a}$ was isolated as colorless oil ( $41.2 \mathrm{mg}, 77 \%$ yield).

3. A general procedure of substrate scope with $\mathrm{Zn}(\text { acac })_{2}$ (Scheme 1 and 2)

[For liquid alcohols]

To an oven dried test tube equipped with a magnetic stirring bar was added $\mathrm{Zn}(\text { acac })_{2}$ ( $1.9 \mathrm{mg}, 0.0075 \mathrm{mmol})$ and powdered MS 5A (200 mg). The tube was capped with a septa, connected to a vacuum line, then an Ar balloon was equipped. BTF $(1.0 \mathrm{~mL})$ was added to the tube, followed by the addition of dimethyl phosphite $2(66.0 \mathrm{mg}, 0.6 \mathrm{mmol})$ and alcohol $1(0.3 \mathrm{mmol})$ by syringe. The reaction mixture was stirred for $18 \mathrm{~h}$ at room temperature. Then, the reaction mixture was diluted with ethyl acetate $(5 \mathrm{~mL})$. The solid material was removed by filtration and washed with ethyl acetate $(5 \mathrm{~mL} * 2)$. The combined organic phase was evaporated and connected to a vacuum line. The ${ }^{1} \mathrm{H}$ NMR was recorded with 1,2,4,5-tetramethyl benzene as internal standard. The crude mixture was purified by column chromatography using hexane/ethyl acetate $=1 / 2$. The product 3 was isolated as colorless oil.

[For solid alcohols]

To an oven dried test tube equipped with a magnetic stirring bar was added $\mathrm{Zn}$ (acac) ${ }_{2}$ (1.9 mg, $0.0075 \mathrm{mmol})$, alcohol 1a $(0.3 \mathrm{mmol})$, and powdered MS 5A (200 mg). The tube was capped with a septa, connected to a vacuum line, then an Ar balloon was equipped. BTF $(1.0 \mathrm{~mL})$ was added to the tube, followed by the addition of dimethyl phosphite 2 ( $66.0 \mathrm{mg}, 0.6 \mathrm{mmol}$ ) by syringe. The reaction mixture was stirred for $18 \mathrm{~h}$ at room 
temperature. Then, the reaction mixture was diluted with ethyl acetate $(5 \mathrm{~mL})$. The solid material was removed by filtration and washed with ethyl acetate $\left(5 \mathrm{~mL}^{*} 2\right)$. The combined organic phase was evaporated and connected to a vacuum line. The ${ }^{1} \mathrm{H}$ NMR was recorded with 1,2,4,5-tetramethyl benzene as internal standard. The crude mixture was purified by column chromatography using hexane/ethyl acetate $=1 / 2$. The product 3 was isolated as colorless oil.

4. A large scale procedure of phosphonylation of alcohol 2a

To an oven dried round bottom flask $(20 \mathrm{~mL})$ equipped with a magnetic stirring bar was added $\mathrm{Zn}(\mathrm{acac})_{2}(7.7 \mathrm{mg}, 0.03 \mathrm{mmol})$ and powdered MS 5A $(800 \mathrm{mg})$. The tube was capped with a septa, connected to a vacuum line, then an Ar balloon was equipped. BTF $(4.0 \mathrm{~mL})$ was added to the tube, followed by the addition of dimethyl phosphite 2 (264.2 $\mathrm{mg}, 2.4 \mathrm{mmol})$ and alcohol $1 \mathrm{a}(120.1 \mathrm{mg}, 1.2 \mathrm{mmol})$ by syringe. The reaction mixture was stirred for $18 \mathrm{~h}$ at room temperature. Then, the reaction mixture was diluted with ethyl acetate $(20 \mathrm{~mL})$. The solid material was removed by filtration and washed with ethyl acetate $\left(10 \mathrm{~mL}^{*} 2\right)$. The combined organic phase was evaporated and connected to a vacuum line. The ${ }^{1} \mathrm{H}$ NMR was recorded with 1,2,4,5-tetramethyl benzene as internal standard. The crude mixture was purified by column chromatography using hexane/ethyl acetate $=1 / 2$. The product $3 \mathrm{a}$ was isolated as colorless oil. (198.8 mg, 93\% yield)

5. A general procedure of substrate scope with $\mathrm{Zn}(\mathrm{TMHD})_{2}$ (Scheme 3 )

[For liquid alcohols]

To an oven dried test tube equipped with a magnetic stirring bar was added $\mathrm{Zn}$ (TMHD) ${ }_{2}$ (1.9 mg, $0.0075 \mathrm{mmol})$ and powdered MS 5A (200 mg). The tube was capped with a septa, connected to a vacuum line, then an Ar balloon was equipped. BTF $(1.0 \mathrm{~mL})$ was added to the tube, followed by the addition of dimethyl phosphite $2(66.0 \mathrm{mg}, 0.6 \mathrm{mmol})$ and alcohol $1(0.3 \mathrm{mmol})$ by syringe. The reaction mixture was stirred for $18 \mathrm{~h}$ at room temperature. Then, the reaction mixture was diluted with ethyl acetate $(5 \mathrm{~mL})$. The solid material was removed by filtration and washed with ethyl acetate ( $5 \mathrm{~mL} * 2)$. The combined organic phase was evaporated and connected to a vacuum line. The ${ }^{1} \mathrm{H}$ NMR was recorded with 1,2,4,5-tetramethyl benzene as internal standard. The crude mixture was purified by column chromatography using hexane/ethyl acetate $=1 / 2$. The product 3 was isolated as colorless oil.

[For solid alcohols]

To an oven dried test tube equipped with a magnetic stirring bar was added $\mathrm{Zn}(\mathrm{TMHD})_{2}$ 
(3.2 mg, $0.0075 \mathrm{mmol})$, alcohol 1a $(0.3 \mathrm{mmol})$, and powdered MS 5A (200 mg). The tube was capped with a septa, connected to a vacuum line, then an Ar balloon was equipped. BTF $(1.0 \mathrm{~mL})$ was added to the tube, followed by the addition of dimethyl phosphite 2 (66.0 $\mathrm{mg}, 0.6 \mathrm{mmol}$ ) by syringe. The reaction mixture was stirred for $18 \mathrm{~h}$ at room temperature. Then, the reaction mixture was diluted with ethyl acetate $(5 \mathrm{~mL})$. The solid material was removed by filtration and washed with ethyl acetate $(5 \mathrm{~mL} * 2)$. The combined organic phase was evaporated and connected to a vacuum line. The ${ }^{1} \mathrm{H}$ NMR was recorded with 1,2,4,5-tetramethyl benzene as internal standard. The crude mixture was purified by column chromatography using hexane/ethyl acetate $=1 / 2$. The product 3 was isolated as colorless oil.

6. A procedure of oxidation of 3 to 4

To an oven dried $10 \mathrm{~mL}$ round bottom flask equipped with magnetic stirring bar was added H-phosphonate 3g (90.1 mg, $0.19 \mathrm{mmol})$. The tube was capped with septa, connected to a vacuum line, the an Ar balloon was equipped. EtOH ( $1 \mathrm{~mL}$ ) was added to make clear solution. Another solution of $\mathrm{I}_{2}(5.1 \mathrm{mg}, 0.02 \mathrm{mmol})$ and TBHP $(30.9 \mathrm{mg}, 0.24$ $\mathrm{mmol})$ in $\mathrm{EtOH}(1 \mathrm{~mL})$ was added to a flask slowly. The reaction solution was stirred for $15 \mathrm{~h}$, at which stage full consumption of $3 \mathrm{~g}$ was confirmed by TLC and ${ }^{31} \mathrm{P}$ NMR. The reaction was quenched by the addition of $\mathrm{NaS}_{2} \mathrm{O}_{3}$ aq. (sat. $1 \mathrm{~mL}$ ), and extracted with DCM ( $5 \mathrm{~mL} * 3$ ). The combined organic phase was dried over $\mathrm{Na}_{2} \mathrm{SO}_{4}$ and solvent was removed in vacuo. to give analytically pure phosphonate $4 \mathrm{~g}$ as a white solid $(91.6 \mathrm{mg})$.

7. Compound data

Cyclohexyl methyl phosphonate 3a

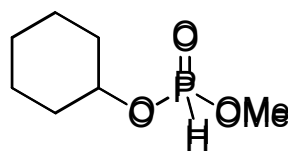

colorless oil, $50.2 \mathrm{mg}$ (94\% yield)

${ }^{1} \mathrm{H}$ NMR $\left(600 \mathrm{MHz}, \mathrm{CDCl}_{3}\right): \delta 6.75\left(1 \mathrm{H}, \mathrm{d}, J_{\mathrm{P}-\mathrm{H}}=693.3 \mathrm{~Hz}\right), 4.43-$

$4.38(1 \mathrm{H}, \mathrm{m}), 3.69\left(3 \mathrm{H}, \mathrm{d}, J_{\mathrm{P}-\mathrm{H}}=11.68 \mathrm{~Hz}\right), 1.94-1.82(2 \mathrm{H}, \mathrm{m}), 1.70-$

$1.65(3 \mathrm{H}, \mathrm{m}), 1.52-1.48(3 \mathrm{H}, \mathrm{m}), 1.32-1.19(2 \mathrm{H}, \mathrm{m}) ;{ }^{13} \mathrm{C} \mathrm{NMR}\left(150 \mathrm{MHz}, \mathrm{CDCl}_{3}\right): \delta$ $76.0\left(\mathrm{~d}, J_{\mathrm{P}-\mathrm{C}}=7.15 \mathrm{~Hz}\right), 51.7\left(\mathrm{~d}, J_{\mathrm{P}-\mathrm{C}}=5.96 \mathrm{~Hz}\right), 33.7\left(\mathrm{~d}, J_{\mathrm{P}-\mathrm{C}}=3.58 \mathrm{~Hz}\right), 33.5\left(\mathrm{~d}, J_{\mathrm{P}-\mathrm{C}}=\right.$ $4.77 \mathrm{~Hz}$ ), 25.0 (s), 23.5 (s) ; ${ }^{31} \mathrm{P}$ NMR $\left(240 \mathrm{MHz}, \mathrm{CDCl}_{3}\right.$ ): $\delta 8.26$ (s) ; IR (neat): 2935, 2859, 1251, 1054, 958, $892 \mathrm{~cm}^{-1}$; HRMS (DART): $\mathrm{m} / \mathrm{z}$ calcd. for $\mathrm{C}_{14} \mathrm{H}_{31} \mathrm{O}_{6} \mathrm{P}_{2}$ $([2 \mathrm{M}+\mathrm{H}]+): 357.1596$; found: 357.1591

Cyclopentyl methyl phosphonate $3 \mathrm{~b}$

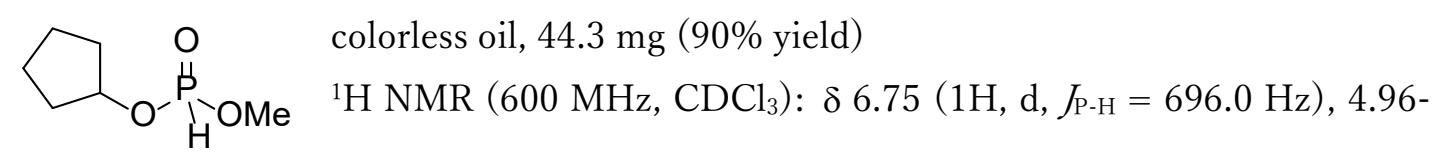


$4.94(1 \mathrm{H}, \mathrm{m}), 3.73\left(3 \mathrm{H}, \mathrm{d}, J_{\mathrm{P}-\mathrm{H}}=11.68 \mathrm{~Hz}\right), 1.80-1.76(6 \mathrm{H}, \mathrm{m}), 1.61-1.58(2 \mathrm{H}, \mathrm{m}) ;{ }^{13} \mathrm{C}$ NMR $\left(150 \mathrm{MHz}, \mathrm{CDCl}_{3}\right): \delta 79.7\left(\mathrm{~d}, J_{\mathrm{P}-\mathrm{C}}=7.22 \mathrm{~Hz}\right), 51.8\left(\mathrm{~d}, J_{\mathrm{P}-\mathrm{C}}=5.78 \mathrm{~Hz}\right), 34.2\left(\mathrm{~d}, J_{\mathrm{P}-}\right.$ $\mathrm{C}=4.33 \mathrm{~Hz}), 34.0\left(\mathrm{~d}, J_{\mathrm{P}-\mathrm{C}}=4.33 \mathrm{~Hz}\right), 22.9(\mathrm{~s}) ;{ }^{31} \mathrm{P} \mathrm{NMR}\left(240 \mathrm{MHz}, \mathrm{CDCl}_{3}\right): \delta 8.61(\mathrm{~s})$; IR (neat): 2964, 2877, 1254, 1053, 966, $800 \mathrm{~cm}^{-1}$; HRMS (DART): $\mathrm{m} / \mathrm{z}$ calcd. for $\mathrm{C}_{12} \mathrm{H}_{27} \mathrm{O}_{6} \mathrm{P}_{2}([2 \mathrm{M}+\mathrm{H}]+): 329.1283$; found: 329.1293

Cycloheptyl methyl phosphonate 3c<smiles>COP(=O)(O)OC1CCCCCC1</smiles>
colorless oil, $56.5 \mathrm{mg}$ ( $98 \%$ yield)

${ }^{1} \mathrm{H}$ NMR $\left(600 \mathrm{MHz}, \mathrm{CDCl}_{3}\right): \delta 6.77\left(1 \mathrm{H}, \mathrm{d}, J_{\mathrm{P}-\mathrm{H}}=690.0 \mathrm{~Hz}\right), 4.64-$ $4.62(1 \mathrm{H}, \mathrm{m}), 3.73\left(3 \mathrm{H}, \mathrm{d}, J_{\mathrm{P}-\mathrm{H}}=11.68 \mathrm{~Hz}\right), 1.98-1.96(2 \mathrm{H}, \mathrm{m}$,$) ,$ 1.80-1.77 (2H, m), 1.67-1.64 (2H, m), 1.55-1.54 (4H, m), 1.43-1.39 (2H, m) ; ${ }^{13} \mathrm{C} \mathrm{NMR}$ $\left(150 \mathrm{MHz}, \mathrm{CDCl}_{3}\right): \delta 78.6\left(\mathrm{~d}, J_{\mathrm{P}-\mathrm{C}}=5.78 \mathrm{~Hz}\right), 51.7\left(\mathrm{~d}, J_{\mathrm{P}-\mathrm{C}}=5.78 \mathrm{~Hz}\right), 35.9\left(\mathrm{~d}, J_{\mathrm{P}-\mathrm{C}}=4.33\right.$ $\mathrm{Hz}), 35.7\left(\mathrm{~d}, J_{\mathrm{P}-\mathrm{C}}=4.33 \mathrm{~Hz}\right), 27.9(\mathrm{~s}), 22.1(\mathrm{~s}) ;{ }^{31} \mathrm{P} \mathrm{NMR}\left(240 \mathrm{MHz}, \mathrm{CDCl}_{3}\right): \delta 8.28(\mathrm{~s})$; IR (neat): 2925, 2859, 1255, 1045, 958, $790 \mathrm{~cm}^{-1}$; HRMS (DART): m/z calcd. for $\mathrm{C}_{16} \mathrm{H}_{35} \mathrm{O}_{6} \mathrm{P}_{2}([2 \mathrm{M}+\mathrm{H}]+)$ : 385.1909; found: 385.1899

(1R,2S,5R)-2-isopropyl-5-methylcyclohexyl methyl phosphonate 3d

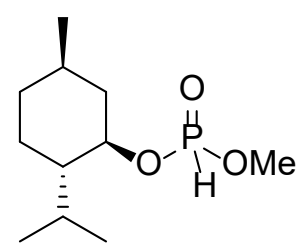
colorless oil, $84.5 \mathrm{mg}$ (98\% yield), $\mathrm{dr}=58 / 42$ ${ }^{1} \mathrm{H}$ NMR $\left(600 \mathrm{MHz}, \mathrm{CDCl}_{3}\right): \delta 6.84\left(1 \mathrm{H}\right.$ for one diastereomer, $\mathrm{d}, J_{\mathrm{P}}$. $\mathrm{H}=692.6 \mathrm{~Hz}), 6.82\left(1 \mathrm{H}\right.$ for the other diastereomer, $\mathrm{d}, J_{\mathrm{P}-\mathrm{H}}=693.6$ $\mathrm{Hz}), 4.30-4.24(1 \mathrm{H}, \mathrm{m}), 3.77(3 \mathrm{H}, \mathrm{d}, \mathrm{J}=12.37 \mathrm{~Hz}), 3.76(3 \mathrm{H}, \mathrm{d}, \mathrm{J}=$ $11.68 \mathrm{~Hz}), 2.18-2.05(2 \mathrm{H}, \mathrm{m}), 1.69-1.67(2 \mathrm{H}, \mathrm{m}), 1.51-1.42(1 \mathrm{H}, \mathrm{m})$, 1.41-1.34 $(1 \mathrm{H}, \mathrm{m}), 1.22(1 \mathrm{H}, \mathrm{q}, \mathrm{J}=11.91 \mathrm{~Hz}), 1.05-0.99(1 \mathrm{H}, \mathrm{m}), 0.93(6 \mathrm{H}, \mathrm{d}, \mathrm{J}=6.87$ $\mathrm{Hz}), 0.90-0.85(1 \mathrm{H}, \mathrm{m}), 0.82(3 \mathrm{H}, \mathrm{d}, \mathrm{J}=6.87 \mathrm{~Hz}) ;{ }^{13} \mathrm{C} \mathrm{NMR}\left(150 \mathrm{MHz}, \mathrm{CDCl}_{3}\right): \delta 78.23$, 78.18, 78.01, 77.96, 51.8, 51.7, 51.6, 51.5, 48.21, 48.18, 43.3, 42.8, 33.85, 33.83, 31.49, 31.47, 25.8, 25.6, 22.8, 21.8, 20.79, 20.76, 15.60, 15.56; ${ }^{31} \mathrm{P}\left(243 \mathrm{MHz}, \mathrm{CDCl}_{3}\right)$ NMR: $\delta$ 8.93, 8.33; IR (neat): 2435, 2118, 1652, 1256, 1181, 1026, 983, 952, 881, 826, 778, $549 \mathrm{~cm}^{-1}$; HRMS (DART): $\mathrm{m} / \mathrm{z}$ calcd. 469.2848 for $\mathrm{C}_{22} \mathrm{H}_{47} \mathrm{O}_{6} \mathrm{P}_{2}([2 \mathrm{M}+\mathrm{H}]+)$ :; found: 469.2774

(1S,2R,4R)-bicyclo[2.2.1] heptan-2-yl methyl phosphonate 3e

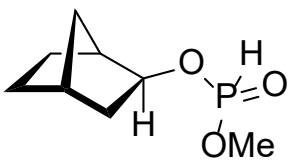
Yellow oil, $55.0 \mathrm{mg}$ (96\% yield), dr 47/53

$1 \mathrm{H}$ NMR (600 MHz, CDCl3): $\delta 6.73$ (1H for one diastereomer, $\mathrm{d}, J_{\mathrm{P}}$ $\mathrm{H}=696.0 \mathrm{~Hz}), 6.73\left(1 \mathrm{H}\right.$ for the other diastereomer, $\mathrm{d}, J_{\mathrm{P}-\mathrm{H}}=696.0$ $\mathrm{Hz}), 4.38(1 \mathrm{H}, \mathrm{q}, J=6.0 \mathrm{~Hz}), 3.71(3 \mathrm{H}$ for one diastereomer, $\mathrm{d}, J=12.4 \mathrm{~Hz}), 3.70(3 \mathrm{H}$ for the other diastereomer, $\mathrm{d}, J=11.7 \mathrm{~Hz}), 2.36(1 \mathrm{H}, \mathrm{d}, J=4.1 \mathrm{~Hz}), 2.26(1 \mathrm{H}, \mathrm{s}), 1.71$ $1.67(1 \mathrm{H}, \mathrm{m}), 1.57-1.54(2 \mathrm{H}, \mathrm{m}), 1.51-1.45(1 \mathrm{H}, \mathrm{m}), 1.42-1.36(1 \mathrm{H}, \mathrm{m}), 1.15(1 \mathrm{H}, \mathrm{d}, \mathrm{JP}-$ 
$\mathrm{H}=8.9 \mathrm{~Hz}), 1.04-0.99(2 \mathrm{H}, \mathrm{m})$; 13C NMR (150 MHz, CDCl3 ): $\delta$ 79.7, 79.62, 79.59, 51.73, 51.69, 43.07, 43.04, 42.83, 42.80, 40.7, 40.64, 40.57, 40.5, 35.27, 35.25, 34.71, 34.68, 27.9, 23.80, 23.78 ; 31P NMR (243 MHz, CDCl3) : $\delta 8.20$; IR (neat): 2967, 2876, 2367, 2331, 1260, 1083, 974, $813 \mathrm{~cm}^{-1}$; HRMS (DART): m/z calcd. 381.1596 for $\mathrm{C}_{16} \mathrm{H}_{31} \mathrm{O}_{6} \mathrm{P}_{2}([2 \mathrm{M}+\mathrm{H}]+): ;$ found: 381.1593

methyl ((1S,2R,4S)-1,7,7-trimethylbicyclo[2.2.1] heptan-2-yl) phosphonate $3 \mathrm{f}$

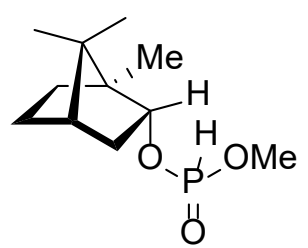
colorless oil, $42.0 \mathrm{mg}$ ( $60 \%$ yield), dr $43 / 57$

1H NMR (600 MHz, CDCl3): $\delta 6.74$ (1H for one diastereomer, $\mathrm{d}$, $\left.J_{\mathrm{P}-\mathrm{H}}=696 \mathrm{~Hz}\right), 6.74\left(1 \mathrm{H}\right.$ for the other diastereomer, $\mathrm{d}, J_{\mathrm{P}-\mathrm{H}}=696$ $\mathrm{Hz}$ ), 4.61-4.55 (1H, m), 3.70 ( $3 \mathrm{H}$ for one diastereomer, d, $J=13.1$ $\mathrm{Hz}$ ), $3.70(3 \mathrm{H}$ for the other diastereomer, $\mathrm{d}, J=13.7 \mathrm{~Hz}$ ), 2.29-2.24 $(1 \mathrm{H}, \mathrm{m}), 1.88-1.86(1 \mathrm{H}, \mathrm{m}), 1.70-1.68(1 \mathrm{H}, \mathrm{m}), 1.63(1 \mathrm{H}, \mathrm{t}, J=4.5 \mathrm{~Hz}), 1.24-1.15(3 \mathrm{H}$, $\mathrm{m}$ ), 0.83 (3H for one diastereomer, s), 0.83 ( $3 \mathrm{H}$ for the other diastereomer, s) ), $0.82(6 \mathrm{H}$ for one diastereomer, s), 0.80 ( $6 \mathrm{H}$ for the other diastereomer, s) ; 13C NMR (150 MHz, CDCl3): $\delta 82.72,82.70,82.67,82.65,51.79,51.75,49.7,49.62,49.6,47.71,47.68,44.85$, 44.83, 37.2, 37.1, 37.03, 27.97, 27.9, 26.39, 26.36, 19.9, 18.7, 13.3, 13.1 ; 31P NMR (243 MHz, CDCl3) : $\delta$ 9.88, 9.72 ; IR (neat): 2958, 2879, 1261, 1058, 967, $793 \mathrm{~cm}^{-1}$; HRMS (DART): $\mathrm{m} / \mathrm{z}$ calcd. 465.2535 for $\mathrm{C}_{22} \mathrm{H}_{43} \mathrm{O}_{6} \mathrm{P}_{2}([2 \mathrm{M}+\mathrm{H}]+)$ :; found: 465.2523

(3S,8S,9S,10R,13R,14S,17R)-10,13-dimethyl-17-((R)-6-methylheptan-2-yl)2,3,4,7,8,9,10,11,12,13,14,15,16,17-tetradecahydro-1H-cyclopenta[a]phenanthren-3-yl methyl phosphonate $3 \mathbf{g}^{6}$

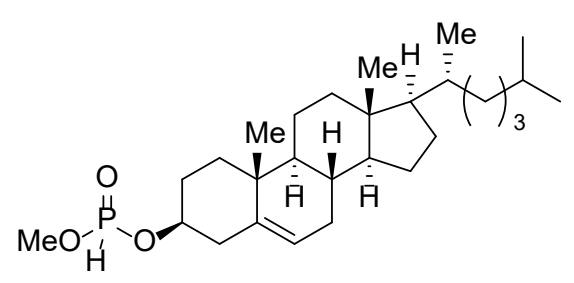

White foam, $138 \mathrm{mg}$ (99\% yield)

1H NMR (600 MHz, CDCl3): $\delta 6.81$ (1H for one diastereomer, $\left.\mathrm{d}, J_{\mathrm{P}-\mathrm{H}}=690 \mathrm{~Hz}\right), 6.81(1 \mathrm{H}$ for the other diastereomer, d, $\left.J_{\mathrm{P}-\mathrm{H}}=690 \mathrm{~Hz}\right), 5.37(1 \mathrm{H}, \mathrm{d}$, $\mathrm{J}=1.4), 4.31-4.24(1 \mathrm{H}, \mathrm{m}), 3.74$ (3H for one diastereomer, $\mathrm{d}, \mathrm{J}=11.7$ ), 3.74 ( $3 \mathrm{H}$ for the other diastereomer, $\mathrm{d}, \mathrm{J}=11.7 \mathrm{~Hz}$ ), $2.45(1 \mathrm{H}$, $\mathrm{t}, \mathrm{J}=12.0 \mathrm{~Hz}), 2.41-2.38(1 \mathrm{H}, \mathrm{m}), 2.00-1.94(3 \mathrm{H}, \mathrm{m}), 1.86-1.78(2 \mathrm{H}, \mathrm{m}), 1.72(1 \mathrm{H}, \mathrm{q}, \mathrm{J}$ $=12.6 \mathrm{~Hz}), 1.58-1.39(7 \mathrm{H}, \mathrm{m}), 1.37-1.29(3 \mathrm{H}, \mathrm{m}), 1.26-1.21(1 \mathrm{H}, \mathrm{m}), 1.16-1.02(7 \mathrm{H}, \mathrm{m})$, 1.00-0.96 (5H, m), $0.89(3 \mathrm{H}, \mathrm{d}, \mathrm{J}=6.2 \mathrm{~Hz}), 0.85-0.81(6 \mathrm{H}, \mathrm{m}), 0.65(3 \mathrm{H}, \mathrm{s}) ; 13 \mathrm{C} \mathrm{NMR}$ (150 MHz, $\mathrm{CDCl} 3): \delta$ 138.9, 123.0, 76.8, 76.7, 56.4, 56.0, 51.8, 51.5, 49.8, 42.12, 42.09, 40.14, 40.12, 39.94, 39.90, 39.5, 39.3, 36.7, 36.2, 36.0, 35.6, 31.7, 31.6, 29.9, 29.8, 29.63, 29.60, 28.0, 27.8, 24.1, 23.7, 22.6, 22.4, 20.8, 19.1, 18.5, 11.7 ; 31P NMR (243 MHz, $\mathrm{CDCl} 3): \delta 8.15$ 
Methyl octan-2-yl phosphonate $3 \mathrm{~h}$<smiles>CCC(C)(C)OP(C)O[PH](C)=O</smiles>
colorless oil, $62.3 \mathrm{mg}$ (>99\% yield), dr 50/50

${ }^{1} \mathrm{H}$ NMR $\left(600 \mathrm{MHz}, \mathrm{CDCl}_{3}\right): \delta 6.73\left(1 \mathrm{H}, \mathrm{d}, J_{\mathrm{P}-\mathrm{H}}=692.6 \mathrm{~Hz}\right), 4.53-$ $4.47(1 \mathrm{H}, \mathrm{m}), 3.70-3.68(3 \mathrm{H}, \mathrm{m}), 1.61-1.58(1 \mathrm{H}, \mathrm{m}), 1.50-1.46(1 \mathrm{H}$, m), 1.33-1.22 (11H, m), 0.82-0.80 (3H, m) ; $\left.{ }^{13} \mathrm{C} \mathrm{NMR} \mathrm{(150} \mathrm{MHz,} \mathrm{CDCl}_{3}\right): \delta 74.64,74.60$, 74.57, $51.5\left(\mathrm{~d}, J_{\mathrm{P}-\mathrm{C}}=5.78 \mathrm{~Hz}\right), 51.4\left(\mathrm{~d}, J_{\mathrm{P}-\mathrm{C}}=5.78 \mathrm{~Hz}\right), 37.34,37.26,37.2,31.43,31.40$, 25.03, 25.01, 24.9, 24.8, 22.31, 22.29, 22.25, 21.92, 21.90, 21.57, 21.55, 13.79, 13.77, 13.7; ${ }^{31} \mathrm{P}$ NMR (240 MHz, $\mathrm{CDCl}_{3}$ ): $\delta 8.64$ (s), 8.31 (s) ; IR (neat): 2924, 2856, 1257, 1058, 962, $801 \mathrm{~cm}^{-1}$; HRMS (DART): $\mathrm{m} / \mathrm{z}$ calcd. for $\mathrm{C}_{9} \mathrm{H}_{22} \mathrm{O}_{3} \mathrm{P}_{1}([\mathrm{M}]+): 209.1307$; found: 209.1303

Methyl (1-phenylethyl) phosphonate 3i<smiles>CC(OP(C)(=O)OCc1ccccc1)c1ccccc1</smiles>
colorless oil, $60.1 \mathrm{mg}$ (>99\% yield) dr 50/50 ${ }^{1} \mathrm{H} \mathrm{NMR}\left(600 \mathrm{MHz}, \mathrm{CDCl}_{3}\right): \delta 7.33-7.23(6 \mathrm{H}, \mathrm{m}), 6.14(1 \mathrm{H}$ for one diasteromer, $\mathrm{d}, J_{\mathrm{P}-\mathrm{H}}$ could not be determined due to overlap of other peak), 6.08 ( $1 \mathrm{H}$ for one diasteromer, $\mathrm{d}, J_{\mathrm{P}-\mathrm{H}}$ could not be determined due to overlap of other peak $), 5.55-5.46(1 \mathrm{H}, \mathrm{m}), 3.65\left(3 \mathrm{H}\right.$ for one diasteromer, $\left.\mathrm{d}, J_{\mathrm{P}-\mathrm{H}}=11.68 \mathrm{~Hz}\right), 3.47$ $\left(3 \mathrm{H}\right.$ for one diastereomer, $\left.\mathrm{d}, J_{\mathrm{P}-\mathrm{H}}=11.68 \mathrm{~Hz}\right), 1.59-1.58(3 \mathrm{H}, \mathrm{m}) ;{ }^{13} \mathrm{C} \mathrm{NMR}(150 \mathrm{MHz}$, $\left.\mathrm{CDCl}_{3}\right): \delta 141.22,141.19,141.0,140.9,128.61,128.59,128.4,128.3,125.84,125.80$, 125.3, $75.5\left(\mathrm{~d}, J_{\mathrm{P}-\mathrm{C}}=5.78 \mathrm{~Hz}\right), 75.2\left(\mathrm{~d}, J_{\mathrm{P}-\mathrm{C}}=5.78 \mathrm{~Hz}\right), 51.63,51.59,24.5,24.18,24.15$; ${ }^{31} \mathrm{P}$ NMR (240 MHz, $\mathrm{CDCl}_{3}$ ): $\delta 8.61$ (s), 8.28 (s) ; IR (neat): 2432, 2353, 1253, 1065, 950, 756, $698 \mathrm{~cm}^{-1}$; HRMS (DART): $\mathrm{m} / \mathrm{z}$ calcd. for $\mathrm{C}_{18} \mathrm{H}_{27} \mathrm{O}_{6} \mathrm{P}_{2}([2 \mathrm{M}+\mathrm{H}]+): 401.1283$; found: 401.1273

Methyl oct-1-en-3-yl phosphonate $\mathbf{3 j}$<smiles>C=CC(C)(C)OP(C)(=O)OC</smiles>
colorless oil, $53.9 \mathrm{mg}$ (87\% yield) dr 50/50

${ }^{1} \mathrm{H}$ NMR $\left(600 \mathrm{MHz}, \mathrm{CDCl}_{3}\right): \delta 6.81$ (1 $\mathrm{H}$ for one diasteromer, d, $J_{\mathrm{P}-\mathrm{H}}$ $=705.0 \mathrm{~Hz}), 6.81\left(1 \mathrm{H}\right.$ for one diasteromer, $\left.\mathrm{d}, J_{\mathrm{P}-\mathrm{H}}=696.1 \mathrm{~Hz}\right), 5.87-$

$5.83(1 \mathrm{H}, \mathrm{m}), 5.34\left(1 \mathrm{H}, \mathrm{d}, J_{\mathrm{P}-\mathrm{H}}=17.18 \mathrm{~Hz}\right), 5.24\left(1 \mathrm{H}, \mathrm{d}, J_{\mathrm{P}-\mathrm{H}}=9.62 \mathrm{~Hz}\right), 4.85-4.83(1 \mathrm{H}$, m), 3.79-3.73 (3H, m), 1.74-1.72 (1H, m), 1.65-1.63 (1H, m), 1.38-1.33 (6H, m), 0.89$0.88(3 \mathrm{H}, \mathrm{m}) ;{ }^{13} \mathrm{C} \mathrm{NMR}\left(150 \mathrm{MHz}, \mathrm{CDCl}_{3}\right): \delta 136.80,136.79,136.5,136.4,117.6,117.3$, 78.64, 78.60, 78.4, 78.3, $51.63\left(\mathrm{~d}, J_{\mathrm{P}-\mathrm{C}}=5.78 \mathrm{~Hz}\right), 51.57\left(\mathrm{~d}, J_{\mathrm{P}-\mathrm{C}}=5.78 \mathrm{~Hz}\right), 35.8,35.8$, 35.73, 35.69, 31.31, 31.27, 31.2, 24.4, 24.3, 22.4, 13.8, 13.82, 13.79; ${ }^{31} \mathrm{P}$ NMR (240 MHz, $\mathrm{CDCl}_{3}$ ): $\delta 8.61$ (s) ; IR (neat): 2930, 2859, 1257, 1082, 955, $792 \mathrm{~cm}^{-1}$; HRMS (DART): $\mathrm{m} / \mathrm{z}$ calcd. for $\mathrm{C}_{18} \mathrm{H}_{39} \mathrm{O}_{6} \mathrm{P}_{2}([2 \mathrm{M}+\mathrm{H}]+)$ : 413.2221; found: 413.2201 
Methyl (3-phenylpropyl) phosphonate 3k

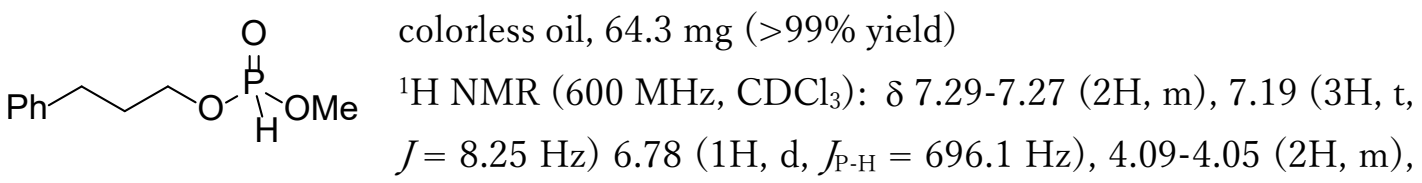
$3.75\left(3 \mathrm{H}, \mathrm{d}, J_{\mathrm{P}-\mathrm{H}}=12.37 \mathrm{~Hz}\right), 2.72(2 \mathrm{H}, \mathrm{t}, J=7.56 \mathrm{~Hz}), 2.03-1.98(2 \mathrm{H}, \mathrm{m}) ;{ }^{13} \mathrm{C} \mathrm{NMR}$ $\left(150 \mathrm{MHz}, \mathrm{CDCl}_{3}\right): \delta 140.7(\mathrm{~s}), 128.5(\mathrm{~s}), 128.4(\mathrm{~s}), 126.1(\mathrm{~s}), 64.9\left(\mathrm{~d}, J_{\mathrm{P}-\mathrm{C}}=5.78 \mathrm{~Hz}\right)$, $51.9\left(\mathrm{~d}, J_{\mathrm{P}-\mathrm{C}}=5.78 \mathrm{~Hz}\right) 31.9\left(\mathrm{~d}, J_{\mathrm{P}-\mathrm{C}}=5.78 \mathrm{~Hz}\right), 31.6(\mathrm{~s}) ;{ }^{31} \mathrm{P}$ NMR $\left(240 \mathrm{MHz}, \mathrm{CDCl}_{3}\right)$ : $\delta 9.83$ (s) ; IR (neat): 3027, 1255, 1051, 963, 803, 745, $699 \mathrm{~cm}^{-1} ;$ HRMS (DART): m/z calcd. for $\mathrm{C}_{10} \mathrm{H}_{16} \mathrm{O}_{3} \mathrm{P}_{1}([\mathrm{M}+\mathrm{H}]+)$ : 215.0837; found: 215.0843

Hexyl methyl phosphonate 31

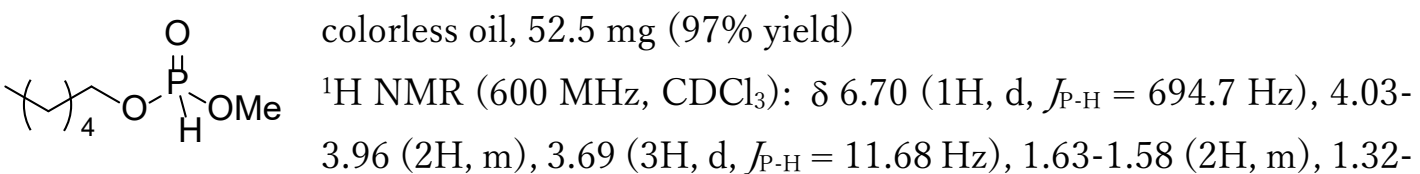
$1.19(6 \mathrm{H}, \mathrm{m}), 0.81(3 \mathrm{H}, \mathrm{t}, J=6.87 \mathrm{~Hz}) ;{ }^{13} \mathrm{C} \mathrm{NMR}\left(150 \mathrm{MHz}, \mathrm{CDCl}_{3}\right): \delta 65.8\left(\mathrm{~d}, J_{\mathrm{P}-\mathrm{C}}=\right.$ $5.78 \mathrm{~Hz}), 51.8\left(\mathrm{~d}, J_{\mathrm{P}-\mathrm{C}}=5.78 \mathrm{~Hz}\right), 31.1(\mathrm{~s}), 30.2\left(\mathrm{~d}, J_{\mathrm{P}-\mathrm{C}}=5.78 \mathrm{~Hz}\right), 25.0(\mathrm{~s}), 22.4(\mathrm{~s})$, 13.8 (s) ; ${ }^{31} \mathrm{P}$ NMR (240 MHz, $\mathrm{CDCl}_{3}$ ): $\delta 9.75$ (s) ; IR (neat): 2958, 2927, 1257, 1051, 950, $781 \mathrm{~cm}^{-1}$; HRMS (DART): $\mathrm{m} / \mathrm{z}$ calcd. for $\mathrm{C}_{7} \mathrm{H}_{18} \mathrm{O}_{3} \mathrm{P}_{1}([\mathrm{M}+\mathrm{H}]+)$ : 181.0994; found: 181.0998

Benzyl methyl phosphonate $3 \mathrm{~m}$

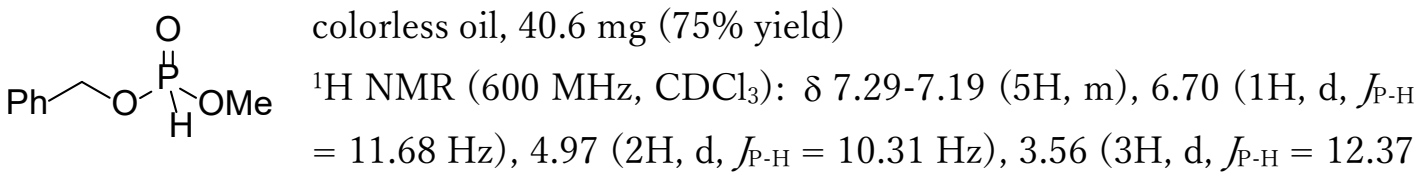
$\mathrm{Hz}) ;{ }^{13} \mathrm{C}$ NMR $\left(150 \mathrm{MHz}, \mathrm{CDCl}_{3}\right): \delta 135.3\left(\mathrm{~d}, \mathrm{~J}_{\mathrm{P}-\mathrm{C}}=5.78 \mathrm{~Hz}\right), 128.4(\mathrm{~s}), 128.4(\mathrm{~s}) 127.7$ $(\mathrm{s}), 67.0\left(\mathrm{~d}, J_{\mathrm{P}-\mathrm{C}}=5.78 \mathrm{~Hz}\right), 51.6\left(\mathrm{~d}, J_{\mathrm{P}-\mathrm{C}}=5.78 \mathrm{~Hz}\right) ;{ }^{31} \mathrm{P}$ NMR $\left(240 \mathrm{MHz}, \mathrm{CDCl}_{3}\right): \delta 9.75$ (s) ; IR (neat): 2350, 1251, 1048, 963, 829, 789, 733, $696 \mathrm{~cm}^{-1}$; HRMS (DART): m/z calcd. for $\mathrm{C}_{16} \mathrm{H}_{23} \mathrm{O}_{6} \mathrm{P}_{2}([2 \mathrm{M}+\mathrm{H}]+)$ : 373.0970 ; found: 373.0980

6-chlorohexyl methyl phosphonate $3 \mathrm{n}$

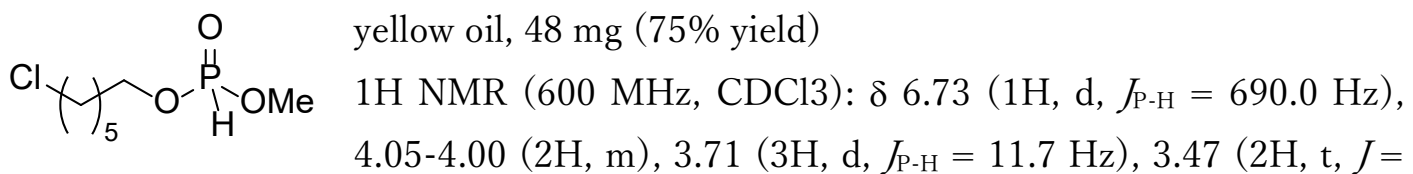
$6.5 \mathrm{~Hz}), 1.74-1.70(2 \mathrm{H}, \mathrm{m}), 1.68-1.63(2 \mathrm{H}, \mathrm{m}), 1.44-1.39(2 \mathrm{H}, \mathrm{m}), 1.38-1.33(2 \mathrm{H}, \mathrm{m})$; 13C NMR (150 MHz, CDCl3): $\delta 65.6\left(\mathrm{~d}, J_{\mathrm{P}-\mathrm{C}}=5.8 \mathrm{~Hz}\right), 51.9,44.8,32.3,30.2\left(\mathrm{~d}, J_{\mathrm{P}-\mathrm{C}}=\right.$ $5.8 \mathrm{~Hz}$ ), 26.3, 24.7 ; 31P NMR (243 MHz, CDCl3) : $\delta 9.80$; IR (neat): 2968, 2894, 2837 , 
1257, 1051, 968, $799 \mathrm{~cm}^{-1}$; HRMS (DART): m/z calcd. 429.1129 for $\mathrm{C}_{14} \mathrm{H}_{33} \mathrm{O}_{6} \mathrm{P}_{2}$ $([2 \mathrm{M}+\mathrm{H}]+): ;$ found: 429.1111

2-(2-(2-ethoxyethoxy) ethoxy)ethyl methyl phosphonate 3o<smiles>CCOCCCOCCOP(C)(=O)OC</smiles>
colorless oil, $40.6 \mathrm{mg}$ (71\% yield) ${ }^{1} \mathrm{H}$ NMR $\left(600 \mathrm{MHz}, \mathrm{CDCl}_{3}\right): \delta 6.81\left(1 \mathrm{H}, \mathrm{d}, J_{\mathrm{P}-\mathrm{H}}=\right.$ $708.64 \mathrm{~Hz}), 4.22-4.18(1 \mathrm{H}, \mathrm{m}), 4.15-4.10(1 \mathrm{H}, \mathrm{m})$, $3.71(3 \mathrm{H}, \mathrm{d}, J=12.37 \mathrm{~Hz}), 3.66(2 \mathrm{H}, \mathrm{t}, \mathrm{J}=4.47 \mathrm{~Hz}), 3.63-3.55(6 \mathrm{H}, \mathrm{m}), 3.54-3.41(2 \mathrm{H}$, m), 3.48-3.44 (2H, m), $1.14(3 \mathrm{H}, \mathrm{t}, \mathrm{J}=6.78 \mathrm{~Hz}) ;{ }^{13} \mathrm{C} \mathrm{NMR}\left(150 \mathrm{MHz}, \mathrm{CDCl}_{3}\right): \delta 70.6$, $70.5\left(\mathrm{~d}, \mathrm{~J}_{\mathrm{c}-\mathrm{p}}=2.89 \mathrm{~Hz}\right), 70.1,69.7,66.6,64.9\left(\mathrm{~d}, \mathrm{~J}_{\mathrm{c}-\mathrm{p}}=5.78 \mathrm{~Hz}\right), 61.7,51.7(\mathrm{~d}, \mathrm{~J}=5.78$ $\mathrm{Hz}), 15.1 ;{ }^{31} \mathrm{P} \mathrm{NMR}\left(240 \mathrm{MHz}, \mathrm{CDCl}_{3}\right.$ ): $\delta 10.31$ (s) ; IR (neat): 2972, 2870, 2359, 1457, 1350, 1257, 974, 810, 548 $\mathrm{cm}^{-1}$; HRMS (DART): $\mathrm{m} / \mathrm{z}$ calcd. for $\mathrm{C}_{16} \mathrm{H}_{23} \mathrm{O}_{6} \mathrm{P}_{2}([\mathrm{M}+\mathrm{H}]+)$ : 257.1154; found: 257.1146

(E)-hex-2-en-1-yl methyl phosphonate 3p

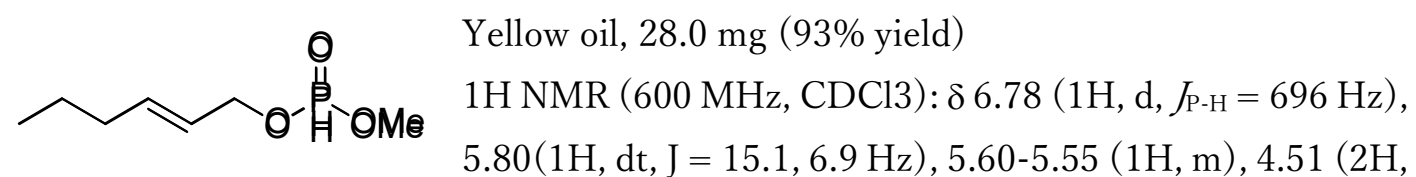
$\mathrm{dd}, \mathrm{J}=10.0,6.5 \mathrm{~Hz}), 3.74(3 \mathrm{H}, \mathrm{d}, \mathrm{J}=12.4 \mathrm{~Hz}), 2.02(2 \mathrm{H}, \mathrm{q}, \mathrm{J}=7.1 \mathrm{~Hz}), 1.39(2 \mathrm{H}, \mathrm{td}, \mathrm{J}$ $=14.8,7.1 \mathrm{~Hz}), 0.88(3 \mathrm{H}, \mathrm{t}, \mathrm{J}=7.6 \mathrm{H} \mathrm{z}) ; 13 \mathrm{C} \mathrm{NMR}(150 \mathrm{MHz}, \mathrm{CDCl} 3): \delta 137.1,124.1$ $\left(\mathrm{d}, J_{\mathrm{P}-\mathrm{C}}=5.75 \mathrm{~Hz}\right), 66.5\left(\mathrm{~d}, J_{\mathrm{P}-\mathrm{C}}=5.75 \mathrm{~Hz}\right), 51.8\left(\mathrm{~d}, J_{\mathrm{P}-\mathrm{C}}=5.75 \mathrm{~Hz}\right), 34.1,21.9,13.5 ; 31 \mathrm{P}$ NMR (243 MHz, CDCl3) : $\delta 9.72$; IR (neat): 2966, 2875, 2367, 1257, 1059, 964, $792 \mathrm{~cm}$ ${ }^{1}$; HRMS (DART): $\mathrm{m} / \mathrm{z}$ calcd. 357.1596 for $\mathrm{C}_{14} \mathrm{H}_{31} \mathrm{O}_{6} \mathrm{P}_{2}([2 \mathrm{M}+1]+)$;; found: 357.1602

( (3a $R, 4 R, 6 S, 6 \mathrm{a} R)$-6-methoxy-2,2-dimethyltetrahydrofuro[3,4- $d][1,3]$ dioxol-4yl) methyl methyl phosphonate $3 q$<smiles>CO[C@H]1O[C@H](COP(O)OC)[C@@H]2OC(C)(C)O[C@H]12</smiles>
colorless oil, $64.3 \mathrm{~g}$ (76\% yield) dr 50/50 ${ }^{1} \mathrm{H}$ NMR $\left(600 \mathrm{MHz}, \mathrm{CDCl}_{3}\right): \delta 6.78 \quad(1 \mathrm{H}$ for one diasteromer, $\left.\mathrm{d}, J_{\mathrm{P}-\mathrm{H}}=705.0 \mathrm{~Hz}\right), 6.77(1 \mathrm{H}$ for one diasteromer, d, $\left.J_{\mathrm{P}-\mathrm{H}}=707.1 \mathrm{~Hz}\right), 4.91(1 \mathrm{H}, \mathrm{s}), 4.65-4.64(1 \mathrm{H}$, m), 4.54-4.53 (1H, m), $4.30(1 \mathrm{H}, \mathrm{t}, J=6.87 \mathrm{~Hz}), 4.06-3.96$ $(2 \mathrm{H}, \mathrm{m}), 3.74\left(3 \mathrm{H}\right.$ for one diasteromer, $\left.\mathrm{d}, \mathrm{J}_{\mathrm{P}-\mathrm{H}}=2.06 \mathrm{~Hz}\right), 3.72(3 \mathrm{H}$ for one diasteromer, $\left.\mathrm{d}, J_{\mathrm{P}-\mathrm{H}}=707.1 \mathrm{~Hz}\right), 3.28-3.24(3 \mathrm{H}, \mathrm{m}), 1.42(3 \mathrm{H}, \mathrm{s}), 1.26(3 \mathrm{H}, \mathrm{s}) ;{ }^{13} \mathrm{C}$ NMR $(150 \mathrm{MHz}$, $\left.\mathrm{CDCl}_{3}\right): \delta 112.6,109.4,109.3,84.98,84.95,84.86,84.8,81.40,81.35,65.52,65.48,65.23$, 65.18, 55.14, 55.12, 52.16, 52.12, 26.3, 24.8; ${ }^{31} \mathrm{P} \mathrm{NMR}\left(240 \mathrm{MHz}, \mathrm{CDCl}_{3}\right): \delta 9.75(\mathrm{~s})$, 9.69 (s) ; IR (neat): 2993, 1258, 1210, 1161, 1090, 1045, 959, 867, 822, $779 \mathrm{~cm}^{-1}$; HRMS 
(DART): $\mathrm{m} / \mathrm{z}$ calcd. for $\mathrm{C}_{10} \mathrm{H}_{20} \mathrm{O}_{7} \mathrm{P}_{1}([\mathrm{M}+\mathrm{H}]+): 283.0947$; found: 283.0935

((2R,3R,4R,5R)-3,4-bis(benzyloxy)-5-methoxytetrahydrofuran-2-yl)methyl methyl phosphonate $3 \mathrm{r}$

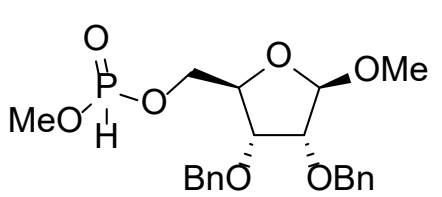

colorless oil, $88.5 \mathrm{mg}$ (68\% yield), dr 50/50

${ }^{1} \mathrm{H}$ NMR (600 MHz, $\left.\mathrm{CDCl}_{3}\right): \delta 7.30-7.19(10 \mathrm{H}, \mathrm{m}), 6.75$ $\left(1 \mathrm{H}\right.$ for one diasteromer, $\left.\mathrm{d}, J_{\mathrm{P}-\mathrm{H}}=707.0 \mathrm{~Hz}\right), 6.74(1 \mathrm{H}$ for one diasteromer, $\left.\mathrm{d}, J_{\mathrm{P}-\mathrm{H}}=707.1 \mathrm{~Hz}\right), 4.82(1 \mathrm{H}, \mathrm{s}), 4.59-4.52$ $(3 \mathrm{H}, \mathrm{m}), 4.37(1 \mathrm{H}, \mathrm{t}, J=11.0 \mathrm{~Hz}), 4.25-4.24(1 \mathrm{H}, \mathrm{m}), 4.21-4.17(1 \mathrm{H}, \mathrm{m}), 4.03-4.02(1 \mathrm{H}$, m), 3.96-3.93 $(1 \mathrm{H}, \mathrm{m}), 3.77(1 \mathrm{H}, \mathrm{t}, J=4.81 \mathrm{~Hz}), 3.69\left(3 \mathrm{H}\right.$ for one diasteromer, $\mathrm{d}, J_{\mathrm{P}-\mathrm{H}}=$ 2.75), 3.66 ( $3 \mathrm{H}$ for one diasteromer, $\mathrm{d}, J_{\mathrm{P}-\mathrm{H}}=2.75$ ), 3.27 ( $3 \mathrm{H}$ for one diasteromer, $\mathrm{s}$ ), 3.26 (3H for one diasteromer, s); ${ }^{13} \mathrm{C}$ NMR $\left(150 \mathrm{MHz}, \mathrm{CDCl}_{3}\right): \delta 137.5,137.39,137.36$, 128.49, 128.46, 128.45, 128.43, 128.39, 128.00, 127.97, 127.92, 127.89, 127.87, 106.4, 106.3, 79.7, 79.61, 79.55, 79.5, 79.2, 79.1, 77.7, 77.5, 72.6, 72.39, 72.37, 66.65, 66.6, 65.95, 65.90, 55.19, 55.18, 51.8, 51.5; $\left.{ }^{31} \mathrm{P} \mathrm{NMR} \mathrm{(240} \mathrm{MHz,} \mathrm{CDCl}_{3}\right): 10.48$ (s), 10.04 (s) ; IR (neat): $2376 \mathrm{~cm}^{-1}, 2333,1106,988,931,869,798,700 \mathrm{~cm}^{-1}$; HRMS (DART): $\mathrm{m} / \mathrm{z}$ calcd. for $\mathrm{C}_{21} \mathrm{H}_{27} \mathrm{O}_{7} \mathrm{P}_{1}([\mathrm{M}]+)$ : 422.1494; found: 422.1493

(2R,3S,5R)-2-((bis (4-methoxyphenyl)(phenyl)methoxy)methyl)-5methoxytetrahydrofuran-3-yl methyl phosphonate 3s

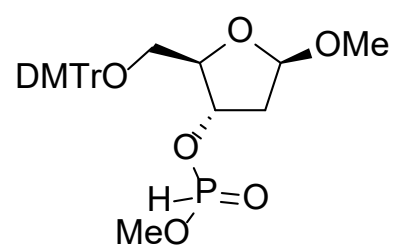

colorless oil, $150.3 \mathrm{mg}$ ( $86 \%$ yield), $\mathrm{dr}=57 / 43$

${ }^{1} \mathrm{H}$ NMR $\left(600 \mathrm{MHz}, \mathrm{CDCl}_{3}\right): \delta 7.34(2 \mathrm{H}, \mathrm{dd}, \mathrm{J}=7.90,1.72$ $\mathrm{Hz}), 7.25-7.18(6 \mathrm{H}, \mathrm{m}), 7.12(1 \mathrm{H}, \mathrm{t}, \mathrm{J}=7.22 \mathrm{~Hz}), 6.75(4 \mathrm{H}$, d, $\mathrm{J}=8.25 \mathrm{~Hz}$ ), 6.69 ( $1 \mathrm{H}$ for one diastereomer, $\mathrm{d}, J_{\mathrm{P}-\mathrm{H}}=705.6$ $\mathrm{Hz}$ ), $6.67\left(1 \mathrm{H}\right.$ for the other diastereomer, $\mathrm{d}, J_{\mathrm{P}-\mathrm{H}}=703.8 \mathrm{~Hz}$ ), $5.09(1 \mathrm{H}, \mathrm{d}, \mathrm{J}=5.50 \mathrm{~Hz}), 4.93-4.86(1 \mathrm{H}, \mathrm{m}), 4.25-4.22(1 \mathrm{H}, \mathrm{m}), 3.70(6 \mathrm{H}, \mathrm{s}), 3.64(3 \mathrm{H}$ for one diastereomer, $\mathrm{d}, \mathrm{J}=12.37 \mathrm{~Hz}$ ), $3.55(3 \mathrm{H}$ for the other diastereomer, $\mathrm{d}, \mathrm{J}=11.00$ $\mathrm{Hz}), 3.33(3 \mathrm{H}, \mathrm{s}), 3.20(1 \mathrm{H}, \mathrm{q}, \mathrm{J}=4.81 \mathrm{~Hz}), 3.12(1 \mathrm{H}, \mathrm{td}, \mathrm{J}=9.97,3.89 \mathrm{~Hz}), 2.41-2.33$ $(1 \mathrm{H}, \mathrm{m}), 2.14-2.10(1 \mathrm{H}, \mathrm{m}) ;{ }^{13} \mathrm{C} \mathrm{NMR}\left(150 \mathrm{MHz}, \mathrm{CDCl}_{3}\right): \delta 158.4,144.60,144.58,135.78$, 125.66, 130.0, 128.1, 127.7, 126.7, 113.1, 105.0, 86.2, 86.1, 83.52, 83.48, 83.3, 83.2, 76.33, 76.29, $76.0,75.9,63.1,63.1,55.11,55.08,51.94,51.90,51.86,40.60,40.58,40.21,40.19 ;{ }^{31} \mathrm{P}(243$ MHz, $\mathrm{CDCl}_{3}$ ) NMR: $\delta$ 9.10; IR (neat): 2439, 2322, 2159, 2026, 1980, 1844, 1701, 1684 , 1669, 1653, 1635, 1576, 1569, 1560, 1558, 1541, 1506, 1436, 1419, 1245, 1212, 1177 , 1033, 974, 830, 729, $700 \mathrm{~cm}^{-1}$; HRMS (DART): m/z calcd. 529.1913 for $\mathrm{C}_{28} \mathrm{H}_{33} \mathrm{O}_{8} \mathrm{P}$ $([\mathrm{M}+\mathrm{H}]+): ;$ found: 529.1910 
(2R,3S,5S)-2-((bis(4-methoxyphenyl) (phenyl)methoxy)methyl)-5-

methoxytetrahydrofuran-3-yl methyl phosphonate $3 \mathrm{t}$

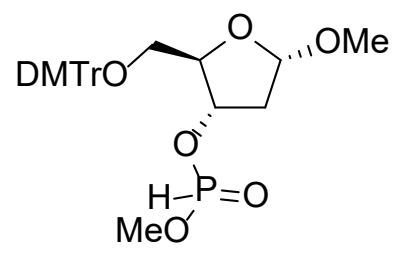

colorless oil, $131.5 \mathrm{mg}$ ( $83 \%$ yield), $\mathrm{dr}=50 / 50$

${ }^{1} \mathrm{H}$ NMR $\left(600 \mathrm{MHz}, \mathrm{CDCl}_{3}\right): \delta 7.46(2 \mathrm{H}, \mathrm{d}, \mathrm{J}=7.37 \mathrm{~Hz}), 7.53$

$(4 \mathrm{H}, \mathrm{d}, \mathrm{J}=8.50 \mathrm{~Hz}), 7.28(2 \mathrm{H}, \mathrm{t}, \mathrm{J}=7.65 \mathrm{~Hz}), 7.22-7.16(1 \mathrm{H}$,

$\mathrm{m}), 6.83(4 \mathrm{H}, \mathrm{d}, \mathrm{J}=8.50 \mathrm{~Hz}), 6.76(1 \mathrm{H}$ for one diastereomer,

$\left.\mathrm{d}, J_{\mathrm{P}-\mathrm{H}}=713.0 \mathrm{~Hz}\right), 6.74\left(1 \mathrm{H}\right.$ for the other diastereomer, d, $J_{\mathrm{P}-}$

$\mathrm{H}=715.0 \mathrm{~Hz}), 5.17-5.12(1 \mathrm{H}, \mathrm{m}), 5.07-5.00(1 \mathrm{H}, \mathrm{m}), 4.22-4.17(1 \mathrm{H}, \mathrm{m}), 3.78(6 \mathrm{H}, \mathrm{s})$, $3.71(3 \mathrm{H}$ for one diastereomer, $\mathrm{d}, \mathrm{J}=11.90 \mathrm{~Hz}$ ), $3.68(3 \mathrm{H}$ for the other diastereomer, $\mathrm{d}$, $\mathrm{J}=11.90 \mathrm{~Hz}), 3.30(3 \mathrm{H}, \mathrm{s}), 3.23(2 \mathrm{H}, \mathrm{d}, \mathrm{J}=3.40 \mathrm{~Hz}), 2.29(2 \mathrm{H}, \mathrm{t}, \mathrm{J}=4.82 \mathrm{~Hz}) ;{ }^{13} \mathrm{C} \mathrm{NMR}$ $\left(150 \mathrm{MHz}, \mathrm{CDCl}_{3}\right): \delta 158.4,144.6,139.5,135.9,135.8,130.0,129.1,128.1,127.75,127.72$, $127.0,126.7,113.0,105.10,105.06,105.0,86.2,86.1,85.9,83.65,83.59,83.5,83.4,81.3,76.9$, $76.7,76.62,76.57,63.7,63.6,63.3,60.3,55.5,55.34,55.32,55.2,55.1,52.1,52.04,51.96,51.9$, 40.17, 40.16, 40.03, 40.00, 21.0, 14.19; ${ }^{31} \mathrm{P}$ (243 MHz, $\mathrm{CDCl}_{3}$ ) NMR: $\delta$ 8.91, 8.61; IR (neat): 2164, 2030, 1943, 1918, 1845, 1734, 1701, 1684, 1636, 1609, 1505, 1452, 1294, 1249 , 1175, 1034, 976, 833, 732, $700 \mathrm{~cm}^{-1}$; HRMS (DART): m/z calcd. 529.1913 for $\mathrm{C}_{28} \mathrm{H}_{33} \mathrm{O}_{8} \mathrm{P}$ $([\mathrm{M}+\mathrm{H}]+):$; found: 529.1911

((2R,3S,5R)-3-(benzyloxy)-5-methoxytetrahydrofuran-2-yl)methyl methyl phosphonate $3 \mathrm{u}$<smiles>CO[C@H]1C[C@@H](Br)[C@H](COP(O)OC)O1</smiles>

colorless oil, $72.1 \mathrm{mg}$ (75\% yield), $\mathrm{dr}=50 / 50$ ${ }^{1} \mathrm{H}$ NMR $\left(600 \mathrm{MHz}, \mathrm{CDCl}_{3}\right): \delta 7.33-7.26(5 \mathrm{H}, \mathrm{m}), 6.83(1 \mathrm{H}$ for one diastereomer, $\left.\mathrm{d}, J_{\mathrm{P}-\mathrm{H}}=705.0 \mathrm{~Hz}\right), 6.82(1 \mathrm{H}$ for the other diastereomer, $\left.\mathrm{d}, \mathrm{J}_{\mathrm{P}-\mathrm{H}}=706.2 \mathrm{~Hz}\right), 5.07(1 \mathrm{H}, \mathrm{d}, \mathrm{J}=2.75 \mathrm{~Hz}), 4.51-4.46(2 \mathrm{H}, \mathrm{m})$, 4.23-4.19 $(1 \mathrm{H}, \mathrm{m}), 4.17-4.04(3 \mathrm{H}, \mathrm{m}), 3.76(3 \mathrm{H}$ for one diastereomer, $\mathrm{d}, \mathrm{J}=2.75 \mathrm{~Hz}$ ), $3.74(3 \mathrm{H}$ for one diastereomer, $\mathrm{d}, \mathrm{J}=3.44 \mathrm{~Hz}$ ), $3.31(3 \mathrm{H}, \mathrm{s}), 2.26-2.22(1 \mathrm{H}, \mathrm{m}), 2.13-$ $2.08(1 \mathrm{H}, \mathrm{m}) ;{ }^{13} \mathrm{C} \mathrm{NMR}\left(150 \mathrm{MHz}, \mathrm{CDCl}_{3}\right): \delta 137.5,128.5,127.9,127.7,105.6,82.4,82.3$, 82.2, 78.92, 78.87, 71.88, 71.85, 66.8, 66.7, 66.32, 66.28, 55.1, 52.00, 51.96, 51.9, 39.30, 39.27; ${ }^{31} \mathrm{P}\left(243 \mathrm{MHz}, \mathrm{CDCl}_{3}\right)$ NMR: $\delta 10.15$, 9.96; IR (neat): 2433, 2152, 2031, 1734, 5171, 1419, 1246, 1043, 974, 830, 736, $699 \mathrm{~cm}^{-1}$; HRMS (DART): $\mathrm{m} / \mathrm{z}$ calcd. 633.2230 for $\mathrm{C}_{28} \mathrm{H}_{43} \mathrm{O}_{12} \mathrm{P}_{2}([2 \mathrm{M}+\mathrm{H}]+)$ :; found: 633.2254

((2R,3S,5S)-3-(benzyloxy)-5-methoxytetrahydrofuran-2-yl)methyl methyl phosphonate $3 \mathrm{v}$ 
<smiles>CO[C@H]1C[C@@H](Br)[C@H](COP(O)OC)O1</smiles>

colorless oil, $75.2 \mathrm{mg}$ (78\% yield), $\mathrm{dr}=50 / 50$

${ }^{1} \mathrm{H}$ NMR $\left(600 \mathrm{MHz}, \mathrm{CDCl}_{3}\right): \delta 7.35-7.28(5 \mathrm{H}, \mathrm{m}), 6.83(1 \mathrm{H}$ for one diastereomer, $\left.\mathrm{d}, J_{\mathrm{P}-\mathrm{H}}=705.0 \mathrm{~Hz}\right), 6.82(1 \mathrm{H}$ for the other diastereomer, d, $\left.J_{\mathrm{P}-\mathrm{H}}=706.2 \mathrm{~Hz}\right), 5.07(1 \mathrm{H}, \mathrm{d}, \mathrm{J}=5.50 \mathrm{~Hz}), 4.60(1 \mathrm{H}, \mathrm{dd}, \mathrm{J}=12.03$, $3.09 \mathrm{~Hz}), 4.49(1 \mathrm{H}, \mathrm{d}, \mathrm{J}=12.37,2.75 \mathrm{~Hz}), 4.25-4.12(2 \mathrm{H}, \mathrm{m}), 4.08-4.02(1 \mathrm{H}, \mathrm{m}), 3.75$ $(3 \mathrm{H}$ for one diastereomer, $\mathrm{d}, \mathrm{J}=11.68 \mathrm{~Hz}$ ), $3.74(3 \mathrm{H}$ for one diastereomer, $\mathrm{d}, \mathrm{J}=12.37$ $\mathrm{Hz}$ ), 3.40 ( $3 \mathrm{H}$ for one diastereomer, s), 3.39 ( $3 \mathrm{H}$ for the other diastereomer, $\mathrm{s}$ ), 2.27-2.20 $(1 \mathrm{H}, \mathrm{m}), 2.05(1 \mathrm{H}, \mathrm{d}, \mathrm{J}=13.75 \mathrm{~Hz}) ;{ }^{13} \mathrm{C} \mathrm{NMR}\left(150 \mathrm{MHz}, \mathrm{CDCl}_{3}\right): \delta 137.7,128.5,128.4$, 128.0, 127.8, 127.7, 105.1, 81.3, 82.22, 82.20, 81.16, 77.86, 71.9, 71.8, 65.41, 65.37, 65.29, 65.25, 55.2, 51.9, 51.83, 51.80, 38.8,; ${ }^{31} \mathrm{P}\left(243 \mathrm{MHz}, \mathrm{CDCl}_{3}\right) \mathrm{NMR}: \delta$ 10.15, 9.96; IR (neat): 2439, 2164, 2028, 1977, 1700, 1257, 1215, 1046, 970, 830, 744, $699 \mathrm{~cm}^{-1}$; HRMS (DART): m/z calcd. 633.2230 for $\mathrm{C}_{28} \mathrm{H}_{43} \mathrm{O}_{12} \mathrm{P}_{2}([2 \mathrm{M}+\mathrm{H}]+)$ :; found: 633.2241

methyl (((2R,3R,4S,5R,6S)-3,4,5-tris(benzyloxy)-6-methoxytetrahydro-2H-pyran-2yl)methyl) phosphonate $3 \mathrm{w}$

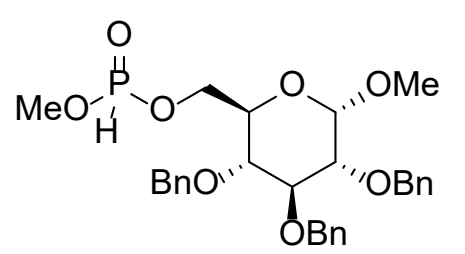

colorless oil, $135.2 \mathrm{mg}$ ( $83 \%$ yield), $\mathrm{dr}=52 / 48$

${ }^{1} \mathrm{H}$ NMR (600 MHz, $\left.\mathrm{CDCl}_{3}\right): \delta$ 7.36-7.27 (15H, m), 6.80 $\left(1 \mathrm{H}\right.$ for one diastereomer, $\left.\mathrm{d}, J_{\mathrm{P}-\mathrm{H}}=708.6 \mathrm{~Hz}\right), 6.75(1 \mathrm{H}$ for the other diastereomer, d, $\left.J_{\mathrm{P}-\mathrm{H}}=708.6 \mathrm{~Hz}\right) 5.00(1 \mathrm{H}, \mathrm{d}, \mathrm{J}=$ $10.31 \mathrm{~Hz}), 4.90(1 \mathrm{H}, \mathrm{dd}, \mathrm{J}=10.31,8,25 \mathrm{~Hz}), 4.82(2 \mathrm{H}, \mathrm{d}$, $\mathrm{J}=11.00 \mathrm{~Hz}), 4.79(2 \mathrm{H}, \mathrm{d}, \mathrm{J}=11.68 \mathrm{~Hz}), 4.67-4.58(3 \mathrm{H}, \mathrm{m}), 4.31-4.20(2 \mathrm{H}, \mathrm{m}), 4.01$ $(1 \mathrm{H}, \mathrm{t}, \mathrm{J}=8.94 \mathrm{~Hz}), 3.73(2 \mathrm{H}, \mathrm{d}, \mathrm{J}=11.68 \mathrm{~Hz}), 3.72(2 \mathrm{H}, \mathrm{d}, \mathrm{J}=12.37 \mathrm{~Hz}), 3.533-3.46$ $(2 \mathrm{H}, \mathrm{m}), 3.38$ (3H for one diastereomer, $\mathrm{s}$ ), 3.36 ( $3 \mathrm{H}$ for the other diastereomer, $\mathrm{s}) ;{ }^{13} \mathrm{C}$ NMR (150 MHz, $\left.\mathrm{CDCl}_{3}\right): \delta 138.5,137.93,137.89,137.8,128.5,128.4,128.1,128.02$, 127.96, 127.91, 127.89, 127.86, 127.6, 98.11, 98.05, 81.8, 79.9, 79.7, 77.1, 77.0, 75.74, 75.72, 75.07, 75.05, 73.42, 73.37, 69.45, 69.41, 69.32, 69.29, 64.73, 64.70, 64.6, 64.5, 55.4, 55.3, 51.78, 51.74; ${ }^{31} \mathrm{P}$ ( $243 \mathrm{MHz}, \mathrm{CDCl}_{3}$ ) NMR: $\delta$ 10.75, 10.59 ; IR (neat): 2159, 2031, 1976, 1943, 1918, 1845, 1825, 1718, 1701, 1696, 1685, 1676, 1635, 1577, 1569, 1550, 1540, 1507, 1459, 1250, 1044, 974, 834, 740, 699 $\mathrm{cm}^{-1} ;$ HRMS (DART): m/z calcd. 543.2148 for $\mathrm{C}_{29} \mathrm{H}_{36} \mathrm{O}_{8} \mathrm{P}([\mathrm{M}+\mathrm{H}]+)$ :; found: 543.2066

1-ethynylcyclohexyl methyl phosphonate $3 \mathrm{x}$<smiles>C#CC1(OP(C)O)CCCCC1</smiles>
colorless oil, $50.2 \mathrm{mg}$ (99\% yield) ${ }^{1} \mathrm{H} \mathrm{NMR}\left(600 \mathrm{MHz}, \mathrm{CDCl}_{3}\right): \delta 7.04\left(1 \mathrm{H}, \mathrm{d}, J_{\mathrm{P}-\mathrm{H}}=702.2 \mathrm{~Hz}\right), 3.79$ $\left(3 \mathrm{H}, \mathrm{d}, J_{\mathrm{P}-\mathrm{H}}=12.4 \mathrm{~Hz}\right), 2.79(1 \mathrm{H}, \mathrm{s}), 2.15-2.04(2 \mathrm{H}, \mathrm{m}), 1.97-1.89$ (2H, m), 1.74-1.70 (2H, m), 1.64-1.58 (2H, m), 1.53-1.51 (1H, m), 
1.36-1.31 (1H, m) ; ${ }^{13} \mathrm{C}$ NMR $\left(150 \mathrm{MHz}, \mathrm{CDCl}_{3}\right): \delta 82.63\left(\mathrm{~d}, J_{\mathrm{P}-\mathrm{C}}=5.78 \mathrm{~Hz}\right), 77.42(\mathrm{~d}$, $\left.J_{\mathrm{P}-\mathrm{C}}=8.67 \mathrm{~Hz}\right), 76.45(\mathrm{~s}), 51.92\left(\mathrm{~d}, J_{\mathrm{P}-\mathrm{C}}=5.78 \mathrm{~Hz}\right), 39.26\left(\mathrm{~d}, J_{\mathrm{P}-\mathrm{C}}=4.33 \mathrm{~Hz}\right), 24.57(\mathrm{~s})$, $22.50\left(\mathrm{~d}, J_{\mathrm{P}-\mathrm{H}}=7.22 \mathrm{~Hz}\right) ;{ }^{31} \mathrm{P}\left(\mathrm{MHz} \mathrm{CDCl}_{3}\right) \mathrm{NMR}: \delta 5.90$; IR (neat): 2938, 2862, 2108 , 1991, 1447, 1262, 1247, 1144, 1078, 1045, 905, 891, 881, 835, 671, 624, $572 \mathrm{~cm}^{-1}$; HRMS (DART): $\mathrm{m} / \mathrm{z}$ calcd. 405.1600 for $\mathrm{C}_{18} \mathrm{H}_{31} \mathrm{O}_{6} \mathrm{P}_{2}([2 \mathrm{M}+\mathrm{H}]+)$ :; found: 405.1561

methyl tert-pentyl phosphonate $3 y$<smiles>CCC(C)(C)O[PH](C)=O</smiles>
colorless oil, $39.9 \mathrm{mg}$ ( $80 \%$ yield) ${ }^{1} \mathrm{H}$ NMR $\left(600 \mathrm{MHz}, \mathrm{CDCl}_{3}\right): \delta 6.77\left(1 \mathrm{H}, \mathrm{d}, J_{\mathrm{P}-\mathrm{H}}=692.0 \mathrm{~Hz}\right), 3.67(3 \mathrm{H}$, $\left.\mathrm{d}, J_{\mathrm{P}-\mathrm{H}}=11.68 \mathrm{~Hz}\right), 1.69(2 \mathrm{H}, \mathrm{q}, J=7.33 \mathrm{~Hz}),, 1.43(6 \mathrm{H}, \mathrm{d}, J=3.44 \mathrm{~Hz})$, $0.90(3 \mathrm{H}, \mathrm{t}, J=7.56 \mathrm{~Hz}) ;{ }^{13} \mathrm{C} \mathrm{NMR}\left(150 \mathrm{MHz}, \mathrm{CDCl}_{3}\right): \delta 86.57(\mathrm{~s}), 51.67\left(\mathrm{~d}, J_{\mathrm{P}-\mathrm{C}}=5.78 \mathrm{~Hz}\right)$, $35.86(\mathrm{~s}), 27.73\left(\mathrm{~d}, J_{\mathrm{P}-\mathrm{C}}=4.33 \mathrm{~Hz}\right), 27.62\left(\mathrm{~d}, J_{\mathrm{P}-\mathrm{C}}=4.33 \mathrm{~Hz}\right), 8.42(\mathrm{~s}) ;{ }^{31} \mathrm{P}$ NMR $(240 \mathrm{MHz}$, $\mathrm{CDCl}_{3}$ ): $\delta 4.60$ (s) ; IR (neat): 2976, 1462, 1373, 1257, 963, $794 \mathrm{~cm}^{-1}$; HRMS (DART): m/z calcd. for $\mathrm{C}_{12} \mathrm{H}_{31} \mathrm{O}_{6} \mathrm{P}_{2}([2 \mathrm{M}+\mathrm{H}]+)$ : 333.1596; found: 333.1592

(8R,9S,13S,14S,17S)-3-hydroxy-13-methyl-7,8,9,11,12,13,14,15,16,17-decahydro-6Hcyclopenta[a] phenanthren-17-yl methyl phosphonate $3 \mathbf{z}$

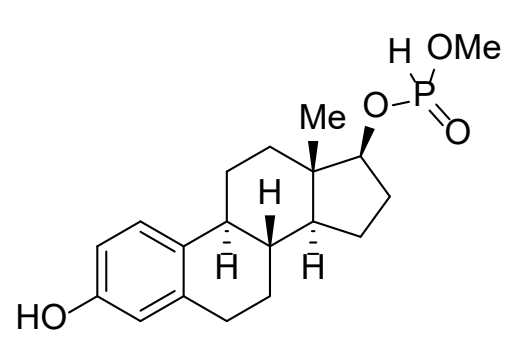
colorless oil, $96.7 \mathrm{mg}$ (93\% yield), $\mathrm{dr}=58 / 42$ ${ }^{1} \mathrm{H}$ NMR $\left(600 \mathrm{MHz}, \mathrm{CDCl}_{3}\right): \delta 7.11(1 \mathrm{H}, \mathrm{d}, \mathrm{J}=2.06 \mathrm{~Hz})$, $6.84\left(1 \mathrm{H}\right.$ for one diastereomer, $\left.\mathrm{d}, \mathrm{J}_{\mathrm{P}-\mathrm{H}}=700.0 \mathrm{~Hz}\right), 6.83(1 \mathrm{H}$ for the other diastereomer, $\left.\mathrm{d}, J_{\mathrm{P}-\mathrm{H}}=699.0 \mathrm{~Hz}\right), 6.68(1 \mathrm{H}, \mathrm{d}$, $\mathrm{J}=2.06 \mathrm{~Hz}), 6.60(1 \mathrm{H}, \mathrm{br}), 4.40-4.35(1 \mathrm{H}, \mathrm{m}), 3.79(3 \mathrm{H}$ for one diastereomer, $\mathrm{d}, \mathrm{J}=11.68 \mathrm{~Hz}), 3.78(3 \mathrm{H}$ for the other diastereomer, $\mathrm{d}, \mathrm{J}=11.68 \mathrm{~Hz}), 2.85-2.76(2 \mathrm{H}, \mathrm{m}), 2.29-2.27(1 \mathrm{H}, \mathrm{m}), 1.96(1 \mathrm{H}, \mathrm{t}, \mathrm{J}=$ $11.68 \mathrm{~Hz}), 1.85-1.82(1 \mathrm{H}, \mathrm{m}), 1.79-1.69(2 \mathrm{H}, \mathrm{m}), 1.49-1.39(3 \mathrm{H}, \mathrm{m}), 1.36-1.27(2 \mathrm{H}, \mathrm{m})$, 1.19-1.14 (1H, m), $0.82(3 \mathrm{H}, \mathrm{s}) ;{ }^{13} \mathrm{C}$ NMR $\left(150 \mathrm{MHz}, \mathrm{CDCl}_{3}\right): \delta 154.1,137.8,131.6,126.3$, 115.3, 112.9, 99.9, 85.4, 85.33, 85.29, 52.01, 51.99, 51.95, 49.03, 49.01, 43.6, 43.4,0, 43.36, 43.3, 38.6, 36.4, 36.3, 29.5, 28.70, 28.69, 28.53, 28.51, 27.1, 23.06, 23.03, 11.62, 11.56 ; ${ }^{31} \mathrm{P}$ ( $243 \mathrm{MHz}, \mathrm{CDCl}_{3}$ ) NMR: $\delta$ 9.20, 9.06; IR (neat): 3225, 2163, 2026, 1972, 1220, 1016, 978, 951, 905, 869, 727, 668, 576, $419 \mathrm{~cm}^{-1}$; HRMS (DART): $\mathrm{m} / \mathrm{z}$ calcd. 701.3372 for $\mathrm{C}_{38} \mathrm{H}_{55} \mathrm{O}_{8} \mathrm{P}_{2}$ $([2 \mathrm{M}+\mathrm{H}]+): ;$ found: 701.3473

Methyl (methyl ((benzyloxy)carbonyl)-L-serinate) phosphonate 3aa 
Cbz-

colorless oil, $78.0 \mathrm{mg}$ (82\% yield), $\mathrm{dr}=50 / 50$

${ }^{1} \mathrm{H}$ NMR $\left(600 \mathrm{MHz}, \mathrm{CDCl}_{3}\right): \delta$ 7.37-7.31 $(5 \mathrm{H}, \mathrm{m})$, one peak $6.18(1 \mathrm{H}$ for one diastereomer, $d, J_{\mathrm{P}-\mathrm{H}}$ could not be determined due to overlap of the other peak), one peak 6.15 ( $1 \mathrm{H}$ for one diastereomer, $\mathrm{d}, J_{\mathrm{P}-\mathrm{H}}$ could not be determined due to overlap of the other peak) $5.84(1 \mathrm{H}, \mathrm{t}, \mathrm{J}=9.97$ $\mathrm{Hz}), 5.16-5.11(2 \mathrm{H}, \mathrm{m}), 4.62-4.58(1 \mathrm{H}, \mathrm{m}), 4.49(1 \mathrm{H}, \mathrm{tt}, \mathrm{J}=13.40,3.67 \mathrm{~Hz}), 4.38(1 \mathrm{H}, \mathrm{dq}, \mathrm{J}$ $=14.43,3.67 \mathrm{~Hz}), 3.80(3 \mathrm{H}, \mathrm{s}), 3.74(3 \mathrm{H}$ for one diastereomer, $\mathrm{d}, \mathrm{J}=10.31 \mathrm{~Hz}), 3.72(3 \mathrm{H}$ for one diastereomer, $\mathrm{d}, \mathrm{J}=11.00 \mathrm{~Hz}) ;{ }^{13} \mathrm{C} \mathrm{NMR}\left(150 \mathrm{MHz}, \mathrm{CDCl}_{3}\right)$ : $\delta 169.3,155.7,136.0$, 135.9, 133.7, 128.5, 128.2, 128.11, 128.09, 67.21, 67.20, 65.4, 65.33, 65.29, 54.37, 56.36, 54.33, 54.29, 53.2, 53.0, 42.9, 52.21, 52.16, 52.14, 52.10; ${ }^{31} \mathrm{P}\left(243 \mathrm{MHz}, \mathrm{CDCl}_{3}\right) \mathrm{NMR}: \delta$ 10.07 ; IR (neat): 2929, 2162, 2034, 1701, 1696, 1685, 1560, 1507, 1497, 1253, 1181, 1029 , 960, 778, 543 $\mathrm{cm}^{-1}$; HRMS (DART): $\mathrm{m} / \mathrm{z}$ calcd. 663.1720 for $\mathrm{C}_{26} \mathrm{H}_{37} \mathrm{~N}_{2} \mathrm{O}_{14} \mathrm{P}_{2}([2 \mathrm{M}+\mathrm{H}]+): ;$ found: 663.1685

(3S,8S,9S,10R,13R,14S,17R)-10,13-dimethyl-17-((R)-4-methylpentan-2-yl)-

2,3,4,7,8,9,10,11,12,13,14,15,16,17-tetradecahydro-1H-cyclopenta[a]phenanthren-3-yl ethyl methyl phosphate $4 \mathrm{~g}$

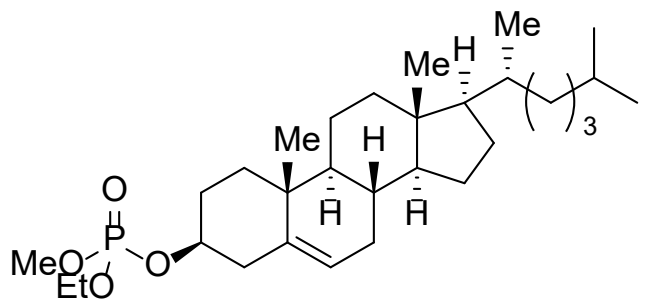
white solid,91.6 $\mathrm{mg}$ (95\% yield) ${ }^{1} \mathrm{H}$ NMR $\left(600 \mathrm{MHz}, \mathrm{CDCl}_{3}\right): \delta 5.39(1 \mathrm{H}, \mathrm{d}, \mathrm{J}=$ $4.12 \mathrm{~Hz}), 4.20(1 \mathrm{H}, \mathrm{dq}, \mathrm{J}=21.82,5.73 \mathrm{~Hz}), 4.13-$ $4.09(2 \mathrm{H}, \mathrm{m}), 3.75(3 \mathrm{H}, \mathrm{dd}, \mathrm{J}=11.34,1.72 \mathrm{~Hz})$, $2.43(2 \mathrm{H}, \mathrm{d}, \mathrm{J}=6.87 \mathrm{~Hz}), 2.02-1.96(3 \mathrm{H}, \mathrm{m}), 1.87-$ $1.81(2 \mathrm{H}, \mathrm{m}), 1.70(1 \mathrm{H}, \mathrm{dt}, \mathrm{J}=29.78,7.39 \mathrm{~Hz}), 1.60-1.33(13 \mathrm{H}, \mathrm{m}), 1.26(2 \mathrm{H}, \mathrm{dd}, \mathrm{J}=19.59$, $12.03 \mathrm{~Hz}), 1.18-1.04(7 \mathrm{H}, \mathrm{m}), 1.03-0.96(5 \mathrm{H}, \mathrm{m}), 0.94-0.91(4 \mathrm{H}, \mathrm{m}), 0.86(6 \mathrm{H}, \mathrm{q}, \mathrm{J}=2.98$ $\mathrm{Hz}), 0.67(3 \mathrm{H}, \mathrm{s}) ;{ }^{13} \mathrm{C} \mathrm{NMR}\left(150 \mathrm{MHz}, \mathrm{CDCl}_{3}\right): \delta 139.3,123.0,78.29,78.25,63.7,63.6,56.6$, 56.1, 54.0, 53.9, 49.9, 42.3, 39.89, 39.86, 39.7, 39.5, 36.8, 36.4, 36.1, 35.7, 31.84, 31.79, 29.55, 29.52, 28.2, 28.0, 25.8, 24.2, 23.8, 22.8, 22.5, 21.0, 19.2, 18.7, 16.12, 16.08, 11.8; ${ }^{31} \mathrm{P}\left(243 \mathrm{MHz}, \mathrm{CDCl}_{3}\right)$ NMR: $\delta$-0.10; IR (neat): 2936, 2159, 2031, 1974, 1465, 1387, 1273, 1052, 971, 905, 827, 760, $732 \mathrm{~cm}^{-1}$; HRMS (DART): m/z calcd. 509.3760 for $\mathrm{C}_{30} \mathrm{H}_{54} \mathrm{O}_{4} \mathrm{P}([\mathrm{M}+\mathrm{H}]+): ;$ found: 509.3703

\section{References}

(1) Gravier-Pelletier, Christine; Milla, Maria; Merrer, Yves Le; Depezay, Jean-Claude, Eur. J. Org. Chem., 2001, 16, $3089-3096$

(2) Le Camus, Corentin; Chassagne, Alexia; Badet-Denisot, Marie-Ange; Badet, Bernard, Tetrahedron Lett, 1998, 39, 287

(3) Giuliano, Robert M.; Villani, Frank J., J. Org. Chem., 1995, 60, 202 
(4) Ding, Yili; Deng, Rilie; Wang, Bingyun, Tetrahedron, 2017, 73, 3848

(5) Kang, Min; Park, Jeonghan; Konradi, Andrei W.; Pedersen, Steven F., J. Org. Chem., 1996, 61, 5528

(6) Y. Kashman, J. Org. Chem. 197237 (6), 912 


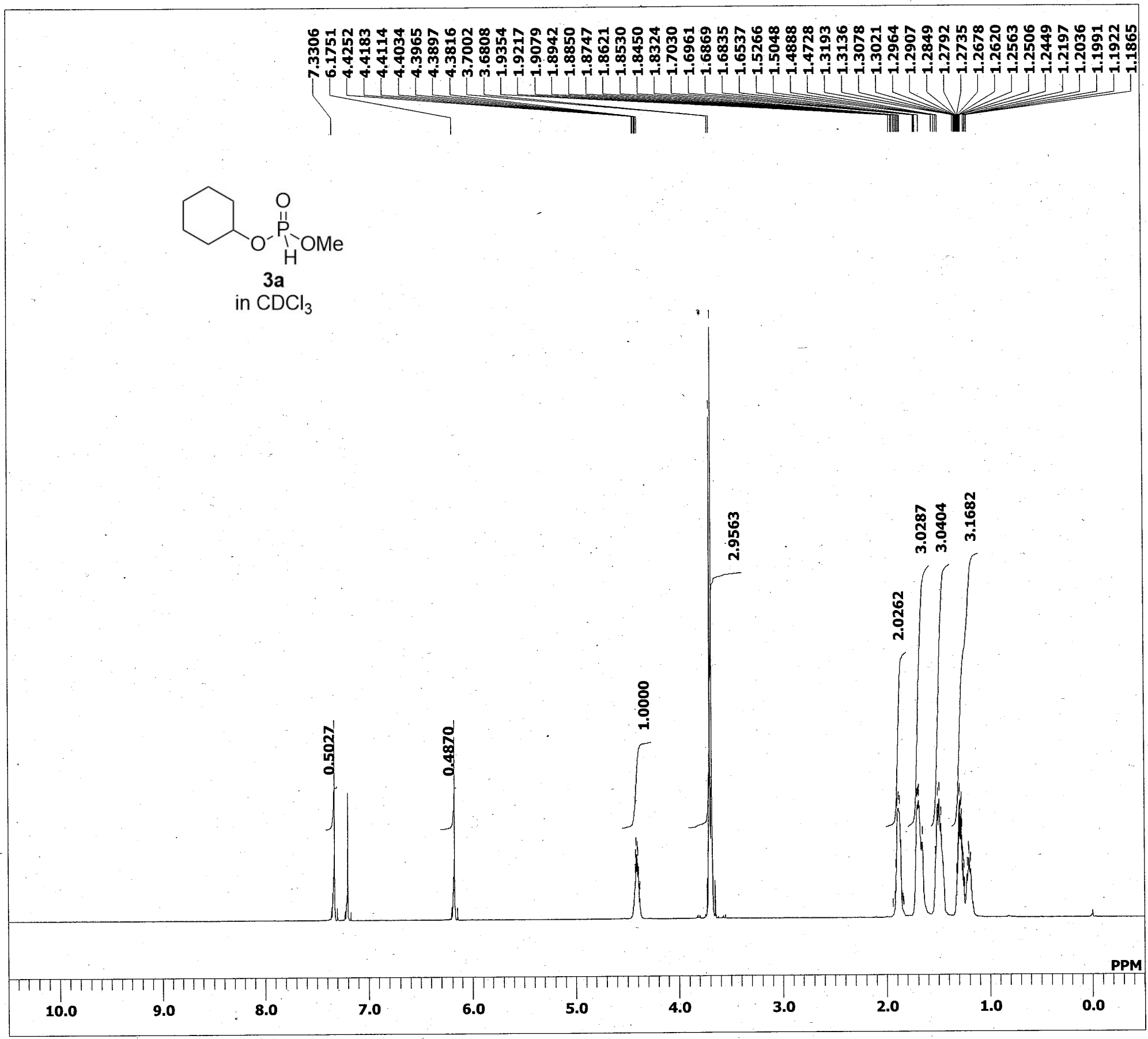

DATTM 05-07-2019 09:33:34

MENUF

1H

OBSET

OBFI

PW1

PREDL

IWT

SPO

DUMMY

FREQU

FLT

DELAY

ACQTM

PD

ADBIT

T1

12

13

EXMOD

EXPCM

IRNUC

IFR

IRSET

IRFIN

IRATN

DFILE

SF

LKSET

LKFIN

LKLEV

LGAIN

LKPHS

CSPED

FILDC

1H -NMR (CDCl3) $\delta:$

$3.69(3 \mathrm{H}, \mathrm{d}, \mathrm{J}=11.68 \mathrm{~Hz})$

1.94-1.83 $(2 \mathrm{H}, \mathrm{m})$,

$1.70-1.65(3 \mathrm{H}, \mathrm{m})$,

1.52-1.48 (3H, m),

$600.17 \mathrm{MHz}$

$5.30 \mathrm{KHz}$

$5.47 \mathrm{~Hz}$
6.39 usec

.

$1.0000 \mathrm{msec}$

13107

13107

$9008.87 \mathrm{~Hz}$

10.88 usec

$1.4549 \mathrm{sec}$

5.0000
16
44

$0.12 \mathrm{~Hz}$

0.00

0.00

100.00

$600.17 \mathrm{MHz}$

$5.30 \mathrm{KHz}$

$72.90 \mathrm{KHz}$

3.8 Hz

0

0

$\mathrm{OHz}$
E:*Personal Folder*Cho*Cho_phosphorylation_st 


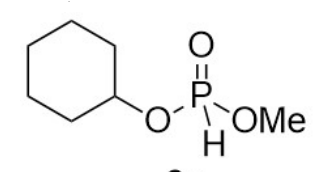

$3 a$

in $\mathrm{CDCl}_{3}$

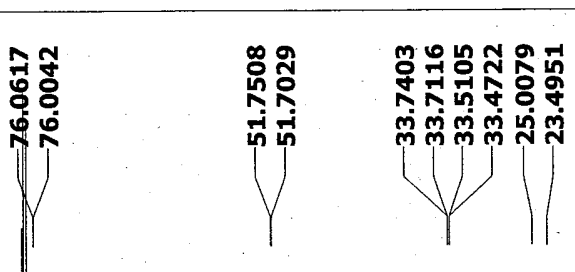

DATIM

MENUF

OBNUC 13

OFR

OBSET

OBFIN

PW1

DEADT

PREDL

IWT

POINT

SPO

TIMES

DUMMY

FREQ

FLT

ACQTM

PD

ADBIT

RGAIN

BF

T2

T3

T4

EXMOD

EXPCM

IRNUC

IFR

IRSET

IRFIN
IRRPW

IRATN

DFILE

SF

LKSET

LKFIN

LKLEV

LGAIN

LKPHS

LKSIG

FILDC

FILDF

13C-NMR (CDCl3) ठ:

$76.03(\mathrm{OH}, \mathrm{d}, \mathrm{J}=7.15 \mathrm{~Hz})$,

$51.73(\mathrm{OH}, d, J=5.96 \mathrm{~Hz})$,

$33.73(\mathrm{OH}, d, J=3.58 \mathrm{~Hz})$,

$33.49(\mathrm{OH}, d, J=4.77 \mathrm{~Hz})$,

$25.01(\mathrm{OH}, \mathrm{s})$,
$23.50(\mathrm{OH}, \mathrm{s})$.
$124.51 \mathrm{MHz}$

$3.45 \mathrm{KHz}$

3.42 usec

0.00 usec

$0.00000 \mathrm{msec}$

26214

26214

302

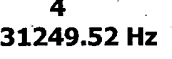

\section{usec}

$2.0000 \mathrm{sec}$

16

50
$0.12 \mathrm{~Hz}$

0.12
0.00
0.00

90.00

ingle_pulse_dec

$495.13 \mathrm{MHz}$

$4.38 \mathrm{KHz}$

$9.64 \mathrm{~Hz}$

$01.50 \mathrm{KHz}$

$-1.8 \mathrm{~Hz}$

0

0

$\mathrm{OHz}$ 


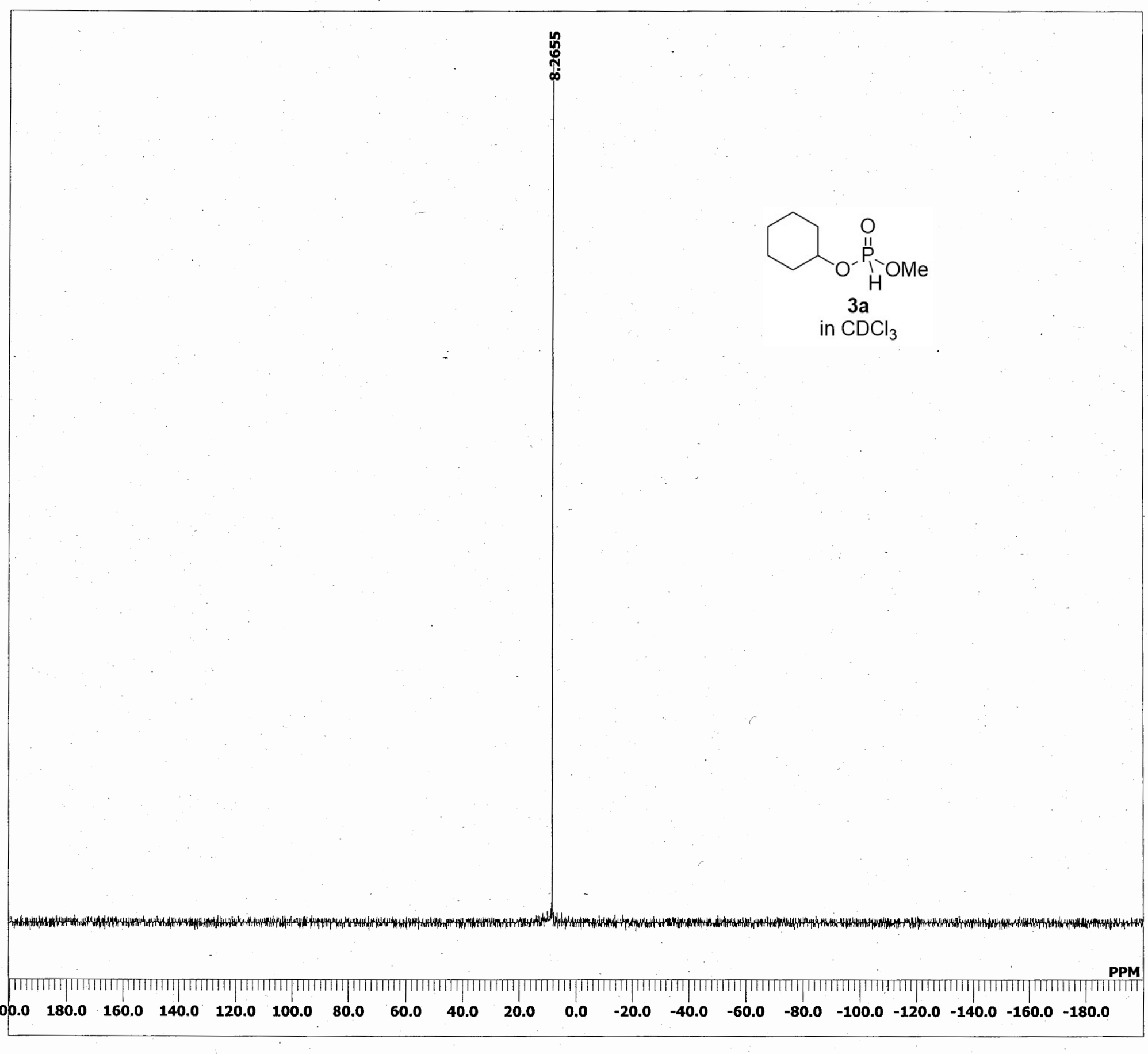

DATIM

MENUF

OBNUC

OFR

OBSET

OBFIN

PWI

DEADT

PREDL

IWT

POINT

SPO

TIMES

DUMMY

FREQ

FLT

ACQTM

PD

ADBIT

RGAIN

BF

T1

13

EXMOD

EXMOD

IRNUC

IFR

IRSET

IRFIN

IRRPW

SF

LKSET

LKFIN

LKLEV

LGAIN

LKPHS

LKSIG

CSPED

FILDC

FILDF
07-2019 10:28:60

$200.43 \mathrm{MHz}$ $2.93 \mathrm{KHz}$

$9.89 \mathrm{~Hz}$

4.12 usec

0.00 usec

$.00000 \mathrm{msec}$

$1.0000 \mathrm{msec}$

26214

26214

218

$142854.95 \mathrm{~Hz}$

20.50 usec $0.1835 \mathrm{sec}$

16

16

$0.12 \mathrm{~Hz}$

0.00

0.00

90.00

single_pulse_dec

495.13 MHz

$4.38 \mathrm{KHz}$

$9.64 \mathrm{~Hz}$

02

E:¥Personal Folder¥Cho¥Cho_phosphorylation_st

$0.00 \mathrm{KHz}$

$0.0 \mathrm{~Hz}$

0

0

$\mathrm{OHz}$ 


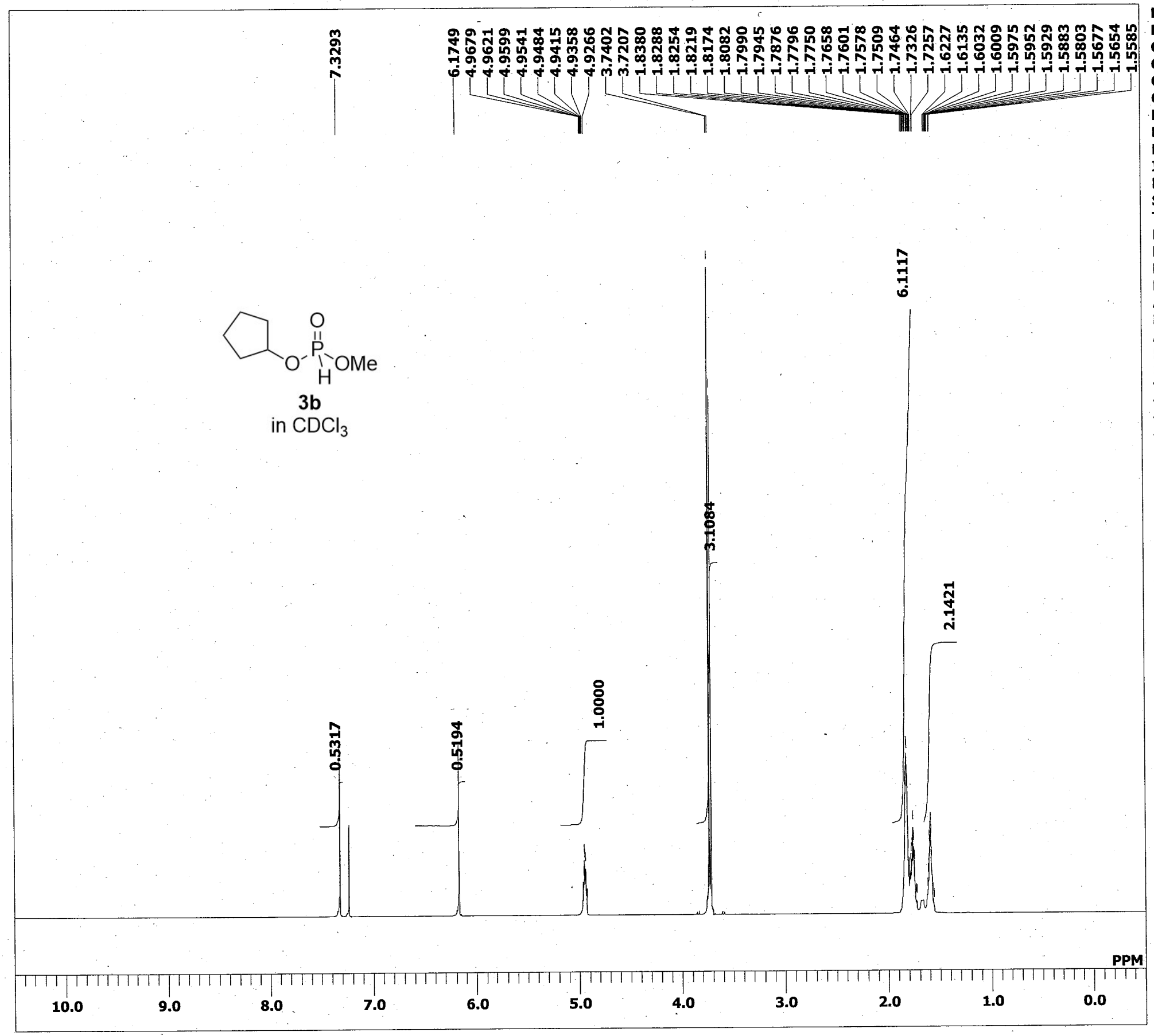

26-12-2019 04:38:30

MENUF

OFR $\quad 600.17 \mathrm{MHz}$

OBSET $\quad 5.30 \mathrm{KHz}$

OBFIN $\quad 5.47 \mathrm{~Hz}$

D.39 usec

PREDI $\quad 0.00000 \mathrm{usec}$

IWT $\quad 1.0000 \mathrm{msec}$

POINT $\quad 13107$

SPO $\quad 13107$

TIMES $\quad 8$

DUMMY 1 .

RREQU $\quad 9008.87 \mathrm{~Hz}$

FLT

10.88 usec
DELAY

PD $\quad \mathbf{5 . 0 0 0 0 \mathrm { sec }}$

ADBIT $\quad 16$

RGAIN $\quad 44$

BF $\quad 0.12 \mathrm{~Hz}$

T1 0.00

T3 $\quad 9.00$

T4 100.00

EXMOD single pulse.ex2

EXPCM $1 \mathrm{H}$

IFR $\quad$ IH $600.17 \mathrm{MHz}$

IRSET $\quad 5.30 \mathrm{KHz}$

IRFIN $\quad 5.47 \mathrm{~Hz}$

IRRPW $\quad 76$ usec

IRATN 0

DFILE E:*Personal Folder*Cho*Cho_phosphorylation_si

SF
LKSET

LKFIN $\quad 53.8 \mathrm{~Hz}$

LKLEV 0

LGAIN - 0

LKPHS $\quad 0$

CSPED $\quad 0 \mathrm{~Hz}$

1H -NMR ( $\mathrm{CDCl} 3) \delta$

4.96-4.94 (1H, m),

$3.73(3 \mathrm{H}, \mathrm{d}, \mathrm{J}=11.68 \mathrm{~Hz})$,

$1.80-1.76(6 \mathrm{H}, \mathrm{m})$,

1.61-1.58 (2H, m). 

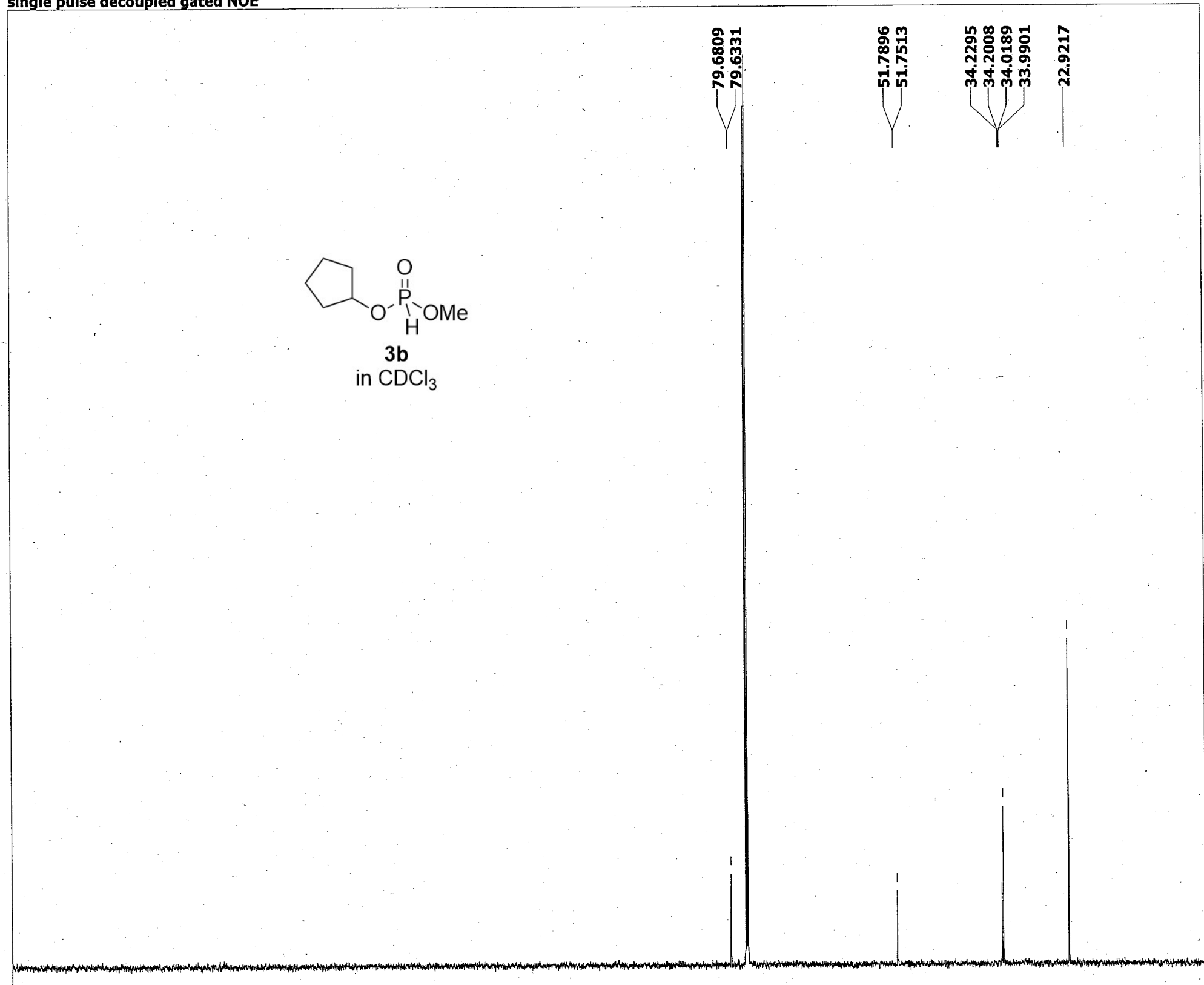

DATIM 26-12-2019 04:50:54

MENUF

OBNUC $13 C$

OFR $\quad 150.92 \mathrm{MHz}$

OBSET $\quad 8.52 \mathrm{KHz}$

OBFIN $\quad 1.74 \mathrm{~Hz}$

PW1 4.02 usec

DEADT $\quad 0.00$ usec

PREDL $\quad 0.00000 \mathrm{msec}$

IWT $\quad 1.0000 \mathrm{msec}$

$\quad 26214$

26214

$\quad 162$

$\begin{array}{ll}4 \\ \text { FREQU } & 37878.21 \mathrm{~Hz}\end{array}$

FLT

DELAY 21.12 usec

ACQTM $0.6921 \mathrm{sec}$

PD $2.0000 \mathrm{sec}$

\begin{tabular}{ll}
2.0000 \\
ADBIT & 16 \\
\hline & 50
\end{tabular}

$\begin{array}{ll}\text { RGAIN } & 50 \\ & 0.12 \mathrm{~Hz}\end{array}$

$\begin{array}{ll}\text { T1 } & 0.00 \\ \text { T1 } & 0.00\end{array}$

0.00
12
$\mathrm{~T} 3$
$\mathrm{~T}$

\begin{tabular}{ll} 
& 90.00 \\
T4 & 100.00 \\
\hline
\end{tabular}

EXMOD single_pulse_dec

EXPCM

IRNUC

IRNUC

$1 \mathrm{H}$

$\quad 600.17 \mathrm{MHz}$

IRFIN $\quad 5.47 \mathrm{~Hz}$

IRRPW $\quad 76$ usec

IRATN 0

DFILE E:*Personal Folder*Cho*Cho_phosphorylation_si

SF

\section{$72.90 \mathrm{KHz}$}

$53.8 \mathrm{~Hz}$

LKLEV $\quad 0$

LKPHS

LKSIG 0

FILDF

13C -NMR ( $\mathrm{CDCl} 3) \delta:$

$79.66(\mathrm{OH}, \mathrm{d}, \mathrm{J}=7.22 \mathrm{~Hz}$ ),

$51.77(0 \mathrm{OH}, \mathrm{d}, \mathrm{J}=5.78 \mathrm{~Hz})$,

$34.22(0 \mathrm{H}, d, J=4.33 \mathrm{~Hz}$ ),

$22.92(\mathrm{OH}, \mathrm{s})$. 
E:*Personal Folder¥Cho*Cho_phosphorylation_sub*sub3b_phos.als

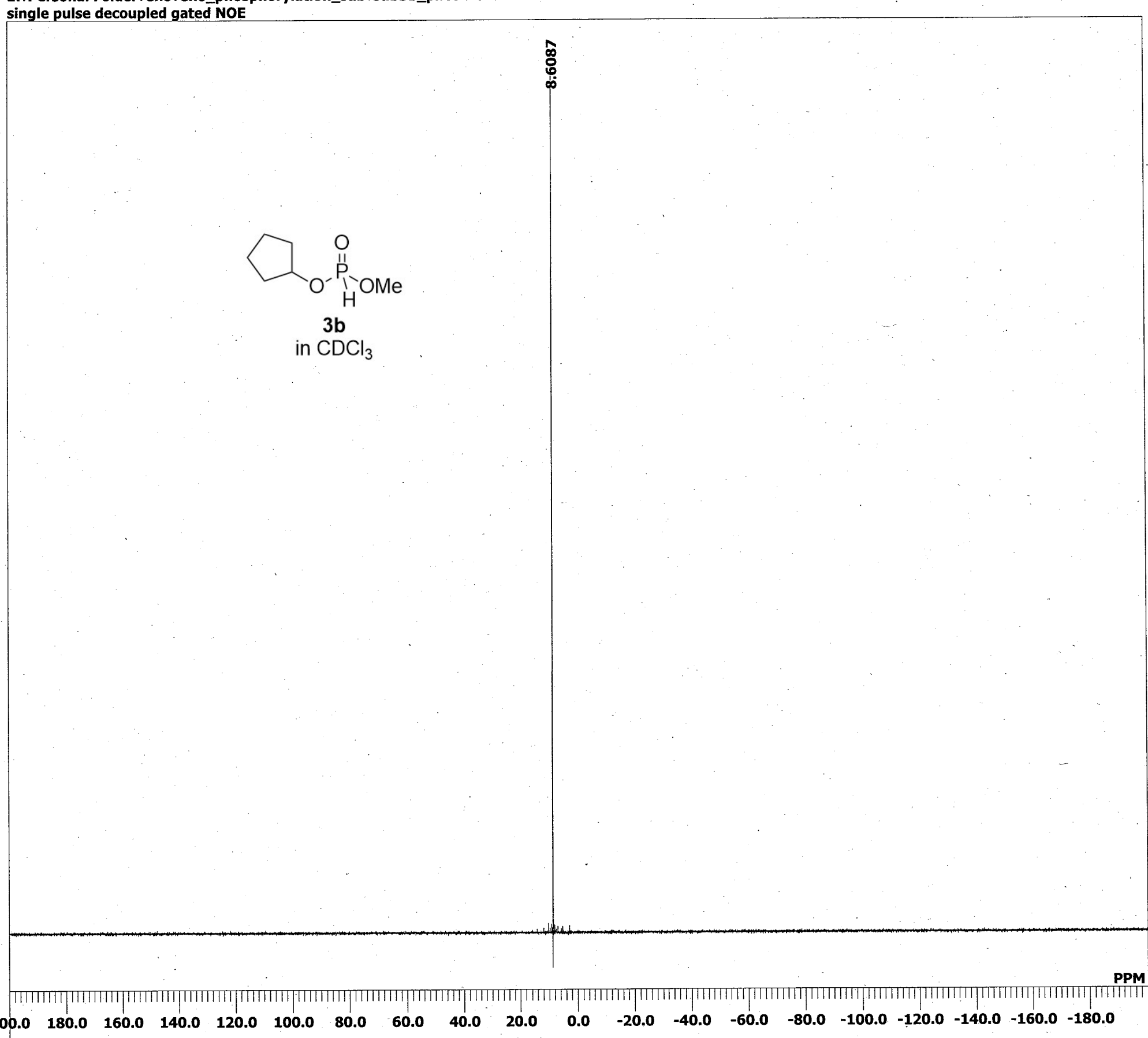

DATIM

MENUC

OBSET

OBFIN

PW1

DEADT

PREDL

IWT

POIN

TIMES

FLT

DELAY

ACQTM

PD

ADBIT

RGA

BF
T1
T2
T3

T3

EXMOD

EXMOD

EXPCM

IRN

IFR

IRSET

IRRPW

IRATN

DFILE

SF

LKSET

LKFIN

LKLEV

LGAIN

LKPHS

LKSIG

CSPED

FILDC
-12-2019 04:41:47

$242.95 \mathrm{MHz}$ $4.04 \mathrm{KHz}$

$1.25 \mathrm{~Hz}$

4.00 usec

$0.0000 \mathrm{msec}$

$0.00000 \mathrm{msec}$

$1.0000 \mathrm{msec}$

26214

72

${ }^{4}$

20.50 usec

$0.1520 \mathrm{sec}$

16
50
0.12

$0.12 \mathrm{~Hz}$

0.00

0.00

0.00

single_pulse_dec

1H

$600.17 \mathrm{MHz}$

$5.30 \mathrm{KHz}$

$5.47 \mathrm{~Hz}$

76 usec

*Personal Folder*Cho*Cho_phosphorylation_sı

$0.00 \mathrm{KHz}$

$0.0 \mathrm{~Hz}$

0

0

$\mathrm{OHz}^{\mathrm{O}}$ 


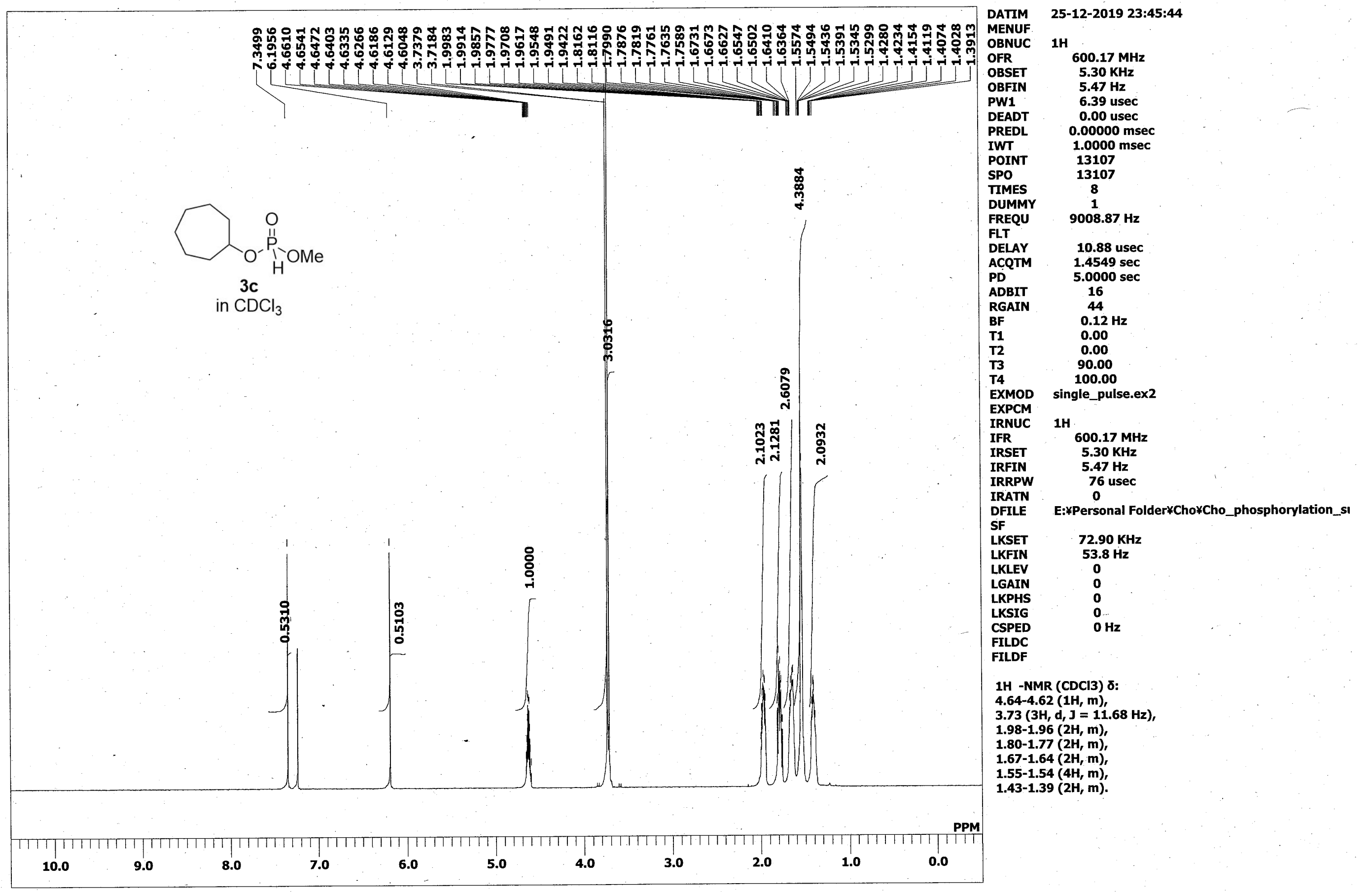



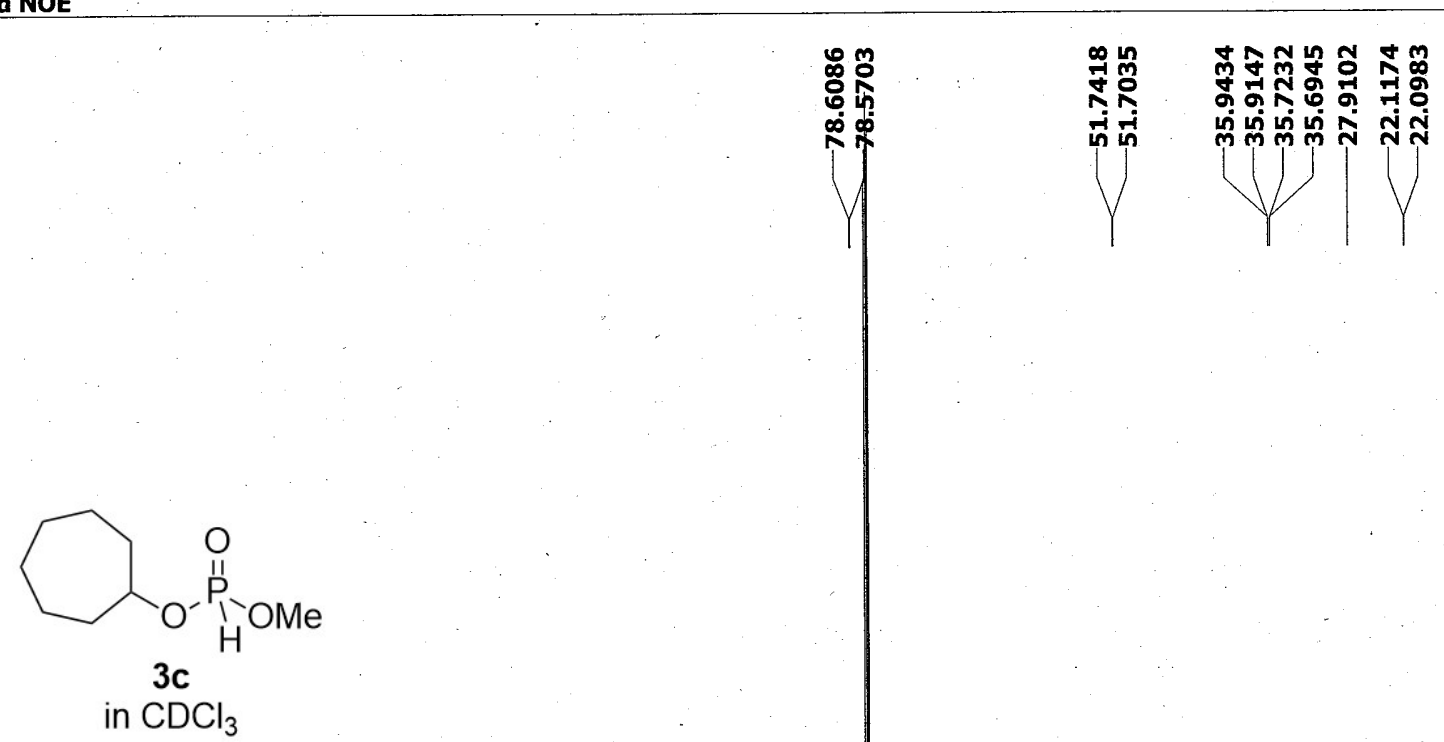

DATIM

MENUF

OBNUC $13 \mathrm{C}$

OFR $\quad 150.92 \mathrm{MHz}$

OBSET $\quad 8.52 \mathrm{KHZ}$

$\begin{array}{ll}8.74 \mathrm{~Hz} \\ \text { PW1 } & 4.02 \text { usec }\end{array}$

DEADT - $\quad 4.02$ usec

PREDL $\quad 0.00$ usec

IWT $\quad 1.0000 \mathrm{msec}$

POINT $\quad 26214$

SPO $\quad 26214$

TIMES $\quad 308$

DUMMY $\quad 3$

$\begin{array}{lc}\text { DUMMY } & \mathbf{4} \\ \text { FREQU } & \mathbf{3 7 8 7 8 . 2 1} \mathrm{Hz}\end{array}$

FREQ

DELAY

DELAY 21.12 usec

PD $2.0000 \mathrm{sec}$

$\begin{array}{ll}\text { ADBIT } & 16 \\ \text { RGAIN } & 50\end{array}$

$\begin{array}{ll}\text { RGAIN } & 50 \\ \text { BF } & 0.12 \mathrm{~Hz}\end{array}$

T1

T3

$\begin{array}{ll}\text { T3 } & 90.00 \\ \text { T4 } & 100.00\end{array}$

EXMOD single_pulse_dec

EXPCM

IRNUC

1H

$\begin{array}{ll}\text { IFR } & 600.17 \mathrm{MHz}\end{array}$

$\begin{array}{ll}\text { IRSET } & 5.30 \mathrm{KHz} \\ \text { IRFIN } & 5.47 \mathrm{~Hz}\end{array}$

IRRPW $76 \mathrm{~Hz}$

IRATN 0

DFILE D:FDocuments and Settings:Shu KOBAYASHI¥De

SF

:*Documents

LKSET $\quad 72.90 \mathrm{KHz}$

LKLEV

LGAIN 0

LKPHS 0

CSPED $\quad 0 \mathrm{~Hz}$

FILDF

$13 \mathrm{C}$-NMR ( $\mathrm{CDCl} 3) \delta$ :

$78.59(0 \mathrm{H}, \mathrm{d}, \mathrm{J}=5.78 \mathrm{~Hz})$

$51.72(\mathrm{OH}, \mathrm{d}, \mathrm{J}=5.78 \mathrm{~Hz})$

$35.93(\mathrm{OH}, \mathrm{d}, \mathrm{J}=4.33 \mathrm{~Hz}$ ),

$27.91\left(\mathrm{OH}_{\mathrm{s}} \mathrm{s}\right)$

$22.10\left(\mathrm{OH}^{\mathrm{s}} \mathrm{s}\right)$

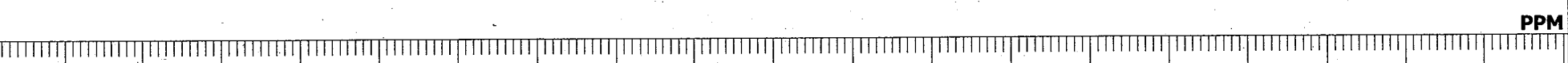
$\begin{array}{lllllllllll}200.0 & 190.0 & 180.0 & 170.0 & 160.0 & 150.0 & 140.0 & 130.0 & 120.0 & 110.0 & 100.0\end{array}$ 
E:*Personal Folder*Cho*Cho_phosphorylation_sub¥sub3c_phos.als

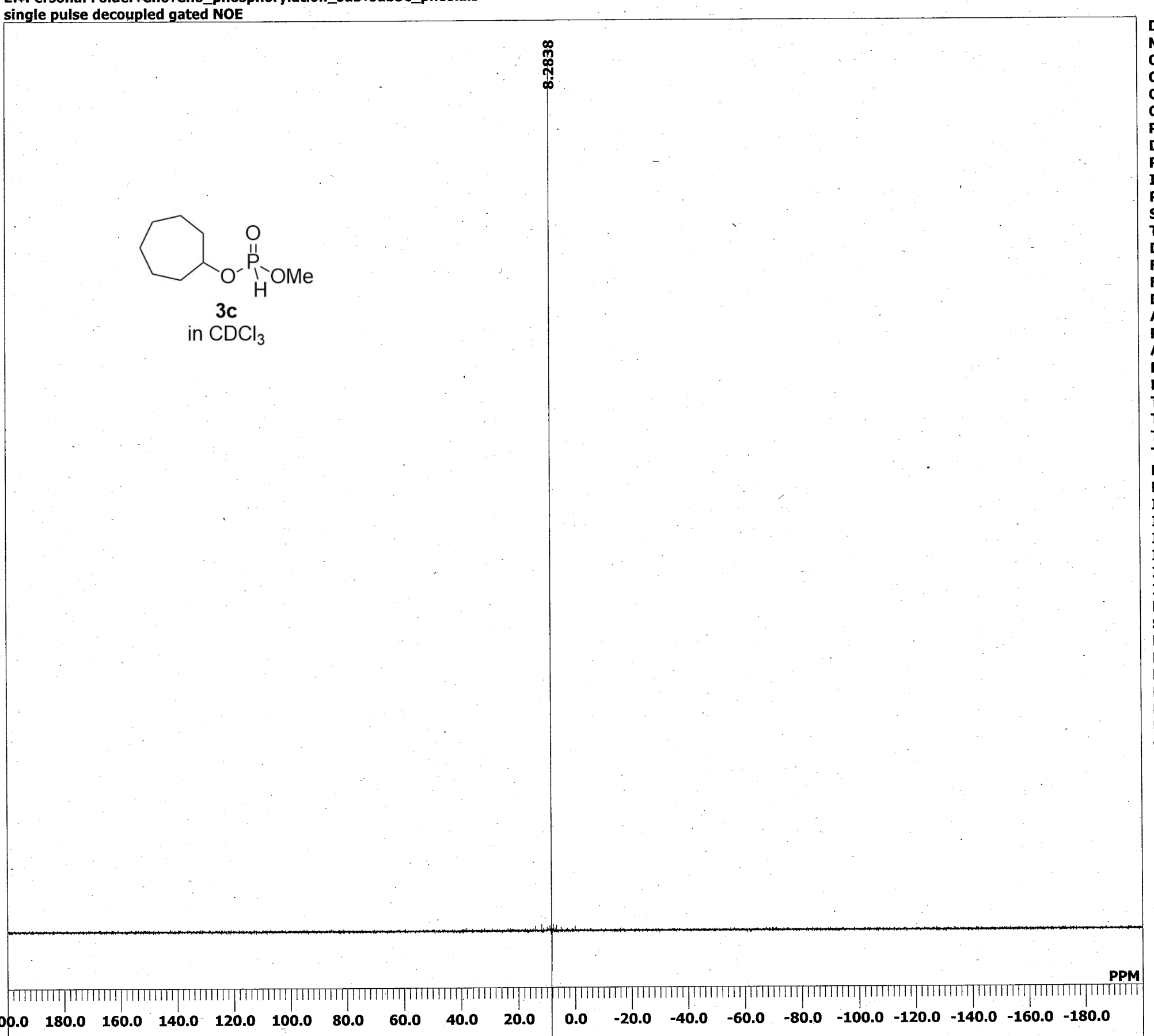

26-12-2019 00:05:22

MENUF

31P

$\quad 242.95 \mathrm{MHz}$

OBSET $\quad 4.04 \mathrm{KHz}$

$1.25 \mathrm{~Hz}$

4.00 usec

$\quad 0.00$ usec

IWT $\quad 10000 \mathrm{msec}$

POINT $\quad 1.0000 \mathrm{msec}$

26214

TIMES $\quad 94$

DUMMY 4

FREQU $\quad 172411.16 \mathrm{~Hz}$

DELAY

DELAY 20.50 usec

PD $\quad 2.0000 \mathrm{sec}$

ADBIT $\quad 16$

BF

T2

13

100.00

single_pulse_dec

EXPCM IH

IFR $\quad 600.17 \mathrm{MHz}$

IRSET $\quad 5.30 \mathrm{KHz}$

IRFIN $\quad 5.47 \mathrm{~Hz}$

IRRPW $\quad 76$ usec

DFIL

SF

KFIN

LKLEV

LGAIN

LKPHS

LKSIG

CSPED

FILDC

FILDF
E:*Perso

$0.00 \mathrm{KHz}$

$0.0 \mathrm{~Hz}$

0

0

$\mathbf{O} \mathrm{Hz}$ 


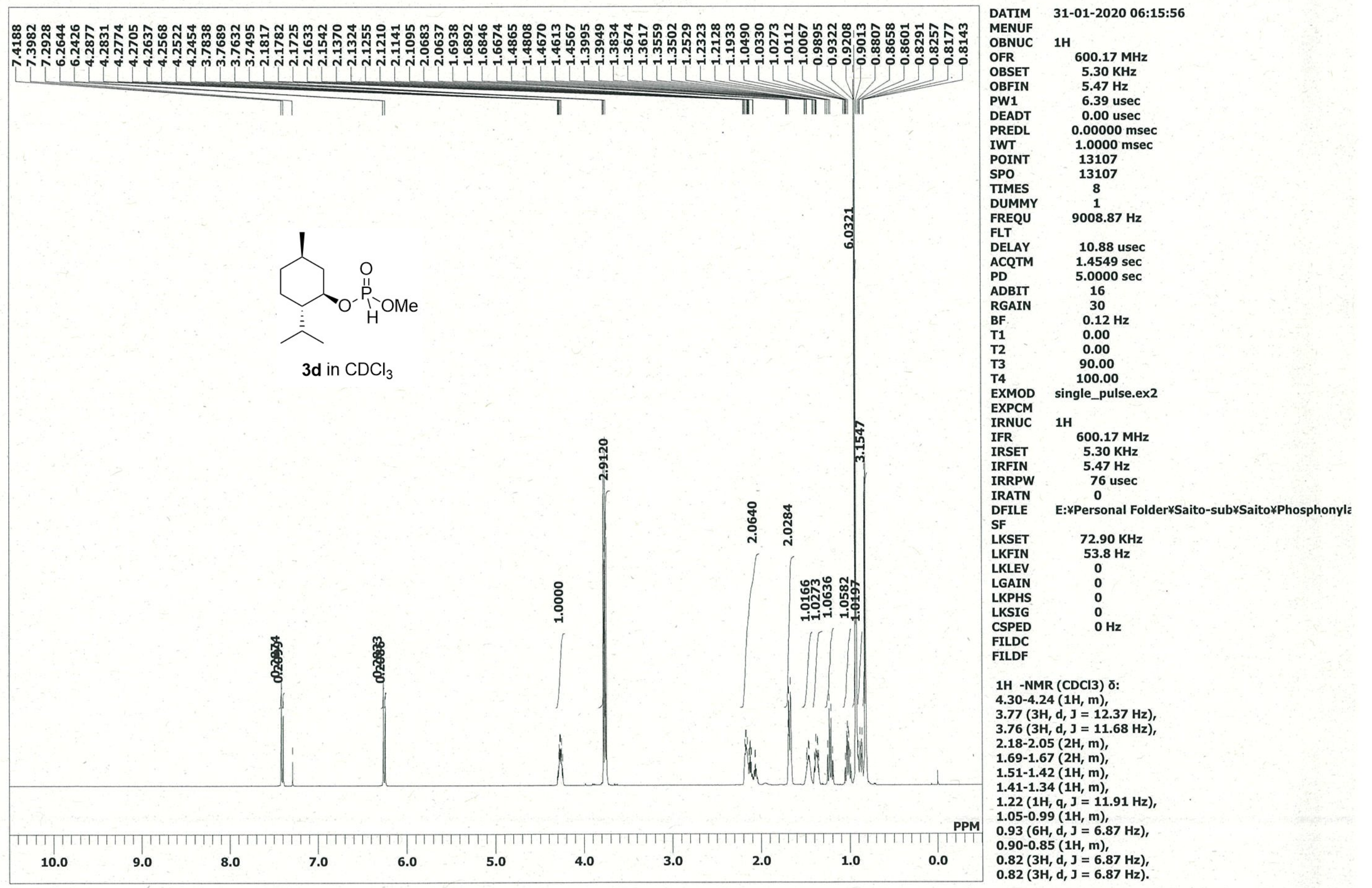


$3 \mathbf{d}$ in $\mathrm{CDCl}_{3}$

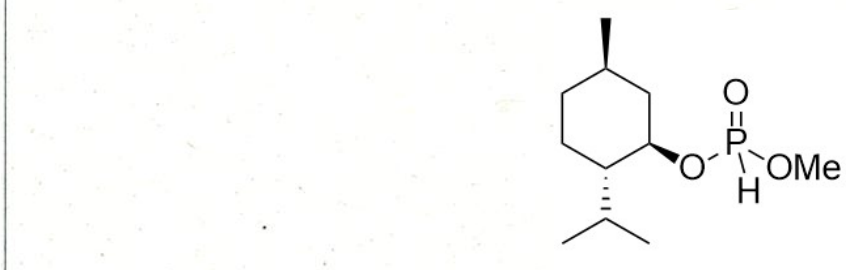

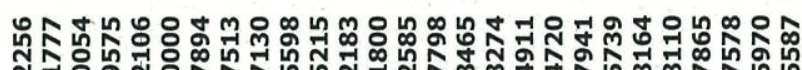

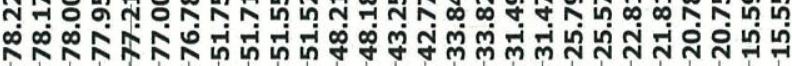
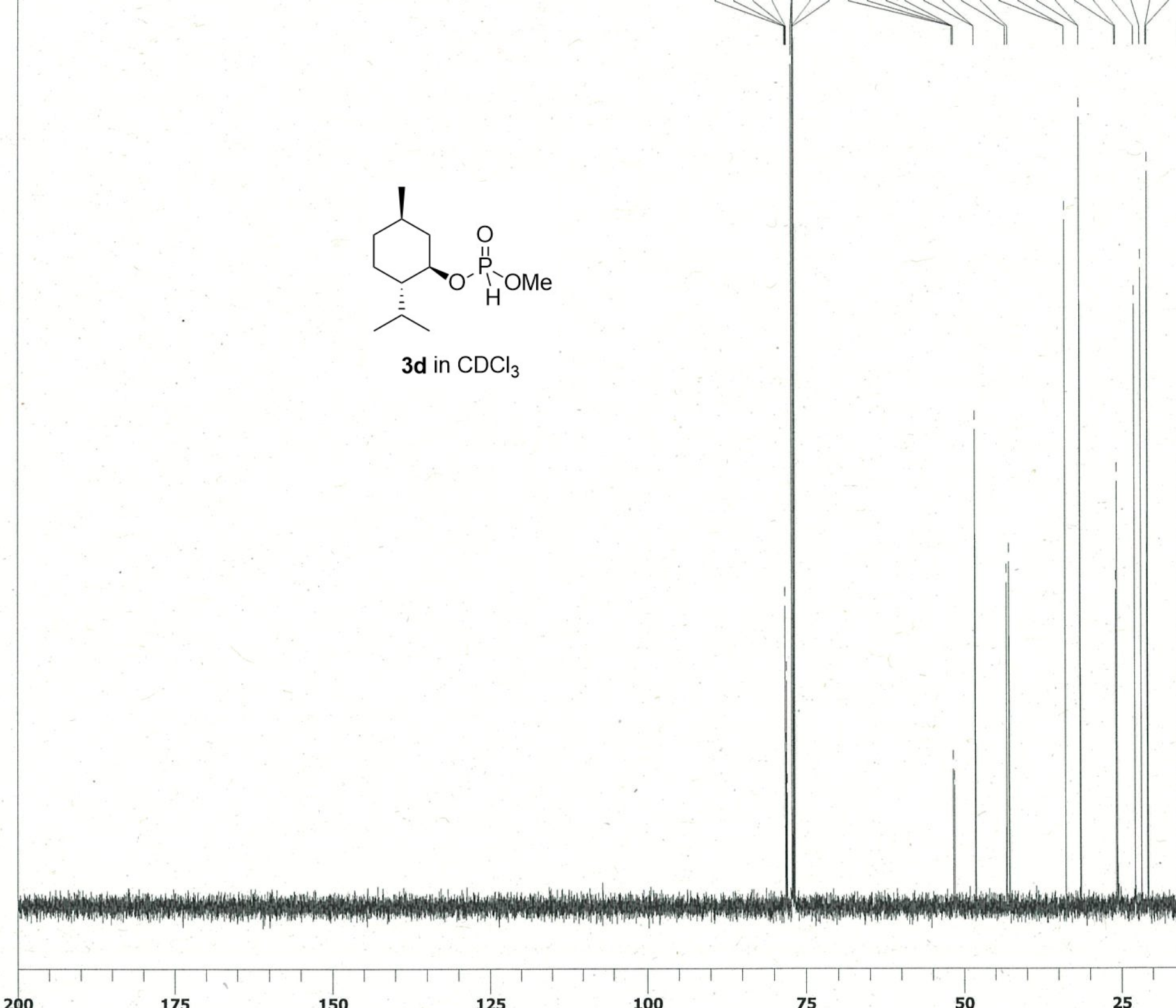

200
DATIM

OBNUC

OBR

OBSET

OBFI

PEADT

DEADT

PREDL

POINT

SPO

TIMES

(IIMES

FLT

DELAY

ACQTM

ADBIT

RGAIN

BF

T1

T3

T4

EXMOD single

EXPCM

IRN

IFR

IRSET

IRFIN

IRRPW

DFILE E:¥Personal Folder*Saito-sub¥Saito¥Phosphony

DF

SF

LKSET

LKLEV

LGAIN

LKPHS

LKSIG

CSPED

FILDC

$13 \mathrm{C}-\mathrm{NMR}(\mathrm{CDCl} 3) \delta$ :

$78.20(\mathrm{OH}, \mathrm{d}, \mathrm{J}=7.22 \mathrm{~Hz})$,

$77.98(\mathrm{OH}, \mathrm{d}, \mathrm{J}=7.22 \mathrm{~Hz})$,

$51.73(\mathrm{OH}, \mathrm{d}, \mathrm{J}=5.78 \mathrm{~Hz}$,

$51.54(\mathrm{OH}, \mathrm{d}, \mathrm{J}=5.78 \mathrm{~Hz})$,

$33.84(\mathrm{OH}, \mathrm{d}, \mathrm{J}=2.89 \mathrm{~Hz})$,
(5.52

$4 \mathrm{~Hz}$

$000 \mathrm{msec}$

26214

161

$37878.21 \mathrm{~Hz}$

12 usec

$2.0000 \mathrm{sec}$

16

$0.12 \mathrm{~Hz}$

0.00

90.00

pulse_dec

$600.17 \mathrm{MH}$

$5.30 \mathrm{KHz}$

$2.47 \mathrm{~Hz}$
$76 \mathrm{sec}$

$53.8 \mathrm{~Hz}$

0

0
$72.90 \mathrm{KHz}$ 
OBNUC

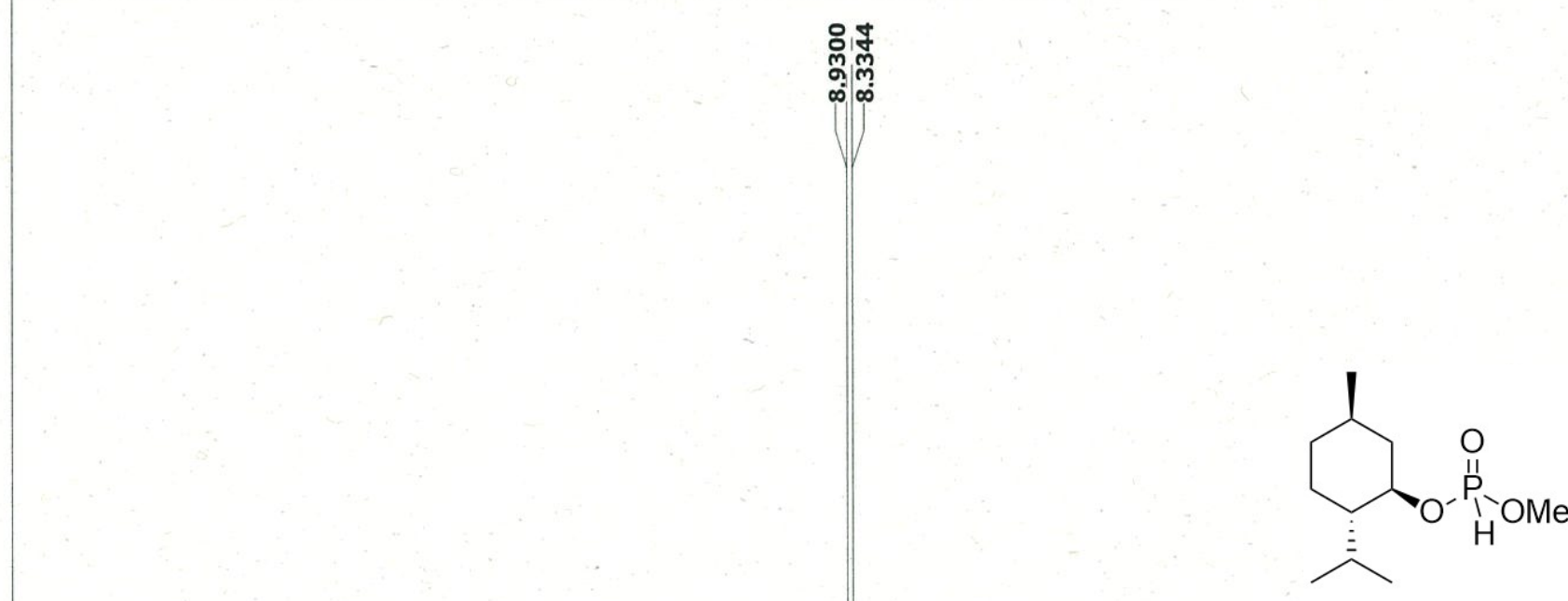

$242.95 \mathrm{MHz}$
$4.04 \mathrm{KHz}$

OBFIN $\quad 1.04 \mathrm{KHz}$

PW1 $\quad 4.00$ usec

0.00 usec

PREDL $\quad 0.00000 \mathrm{msec}$

IWT $\quad 1.0000 \mathrm{msec}$

POINT 26214

TIMES $\quad 45$

DUMMY $\quad 4$.

FREQU $172411.16 \mathrm{~Hz}$

FLT

DELAY 20.50 usec

ACQTM $\quad 0.1520 \mathrm{sec}$

ADBIT $\quad 2.0000 \mathrm{sec}$

RGAIN $\quad 16$

BF $\quad 0.12 \mathrm{~Hz}$

3d in $\mathrm{CDCl}_{3}$

T2

T3

EXMOD single pulse dec

EXPCM

EXPCM

IFN

$1 \mathrm{H}$

IFR

IRSET
IRFIN

IRFIN
IRRPW

IRATN

DFILE

DFIL

LFSET

LKFIN

LKLEV

LGAIN

LKPHS

LKSIG

FILDC

FILDF

$600.17 \mathrm{MHz}$

$5.37 \mathrm{~Hz}$

$76 \mathrm{Hzec}$

E:*Personal Folder¥Saito-sub¥Saito*Phosphonyla

$0.00 \mathrm{KHz}$

0

0

$\mathbf{O H z}$ 


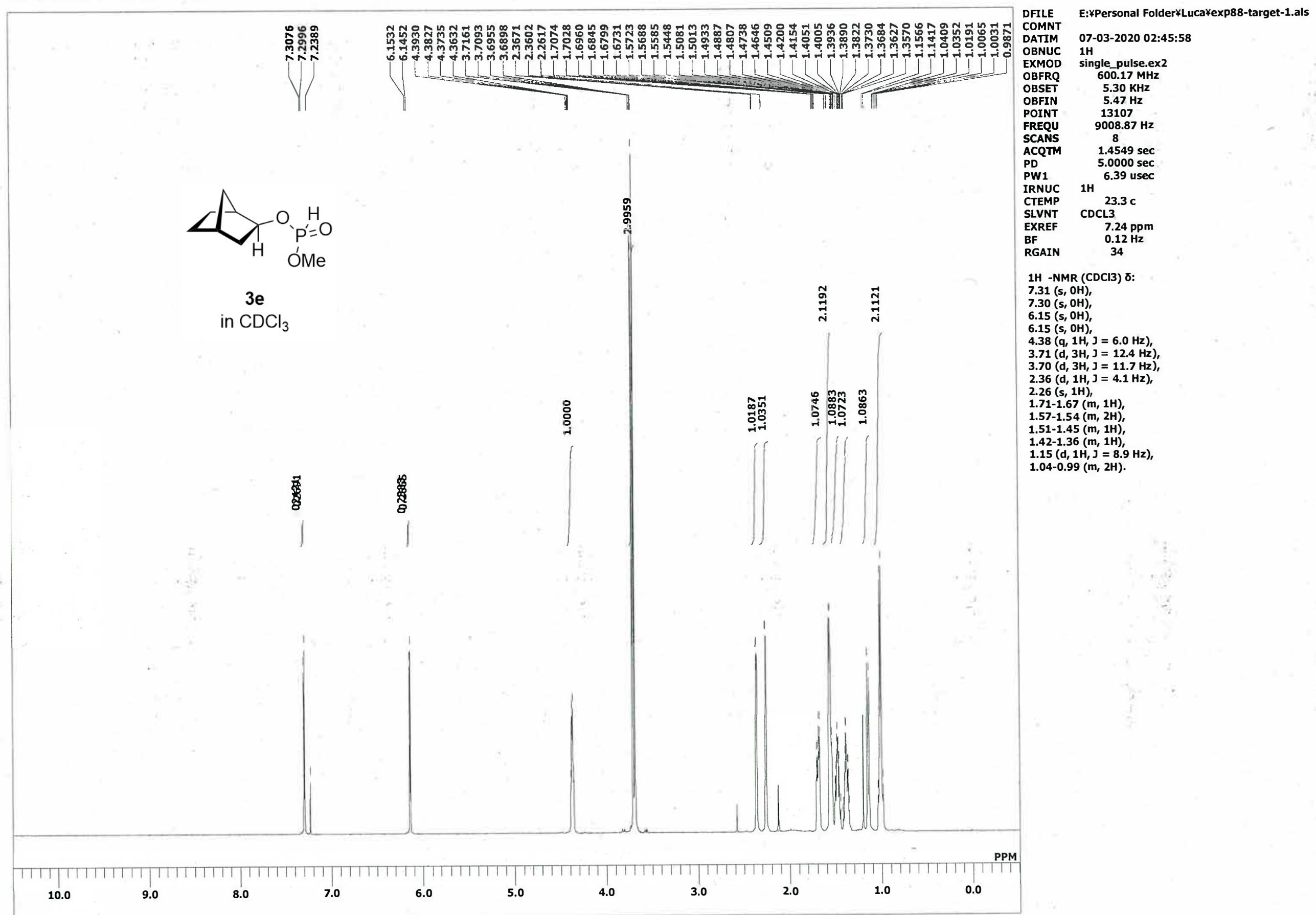




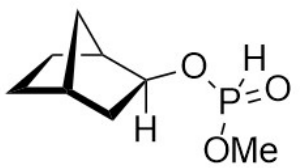

$3 e$

in $\mathrm{CDCl}_{3}$

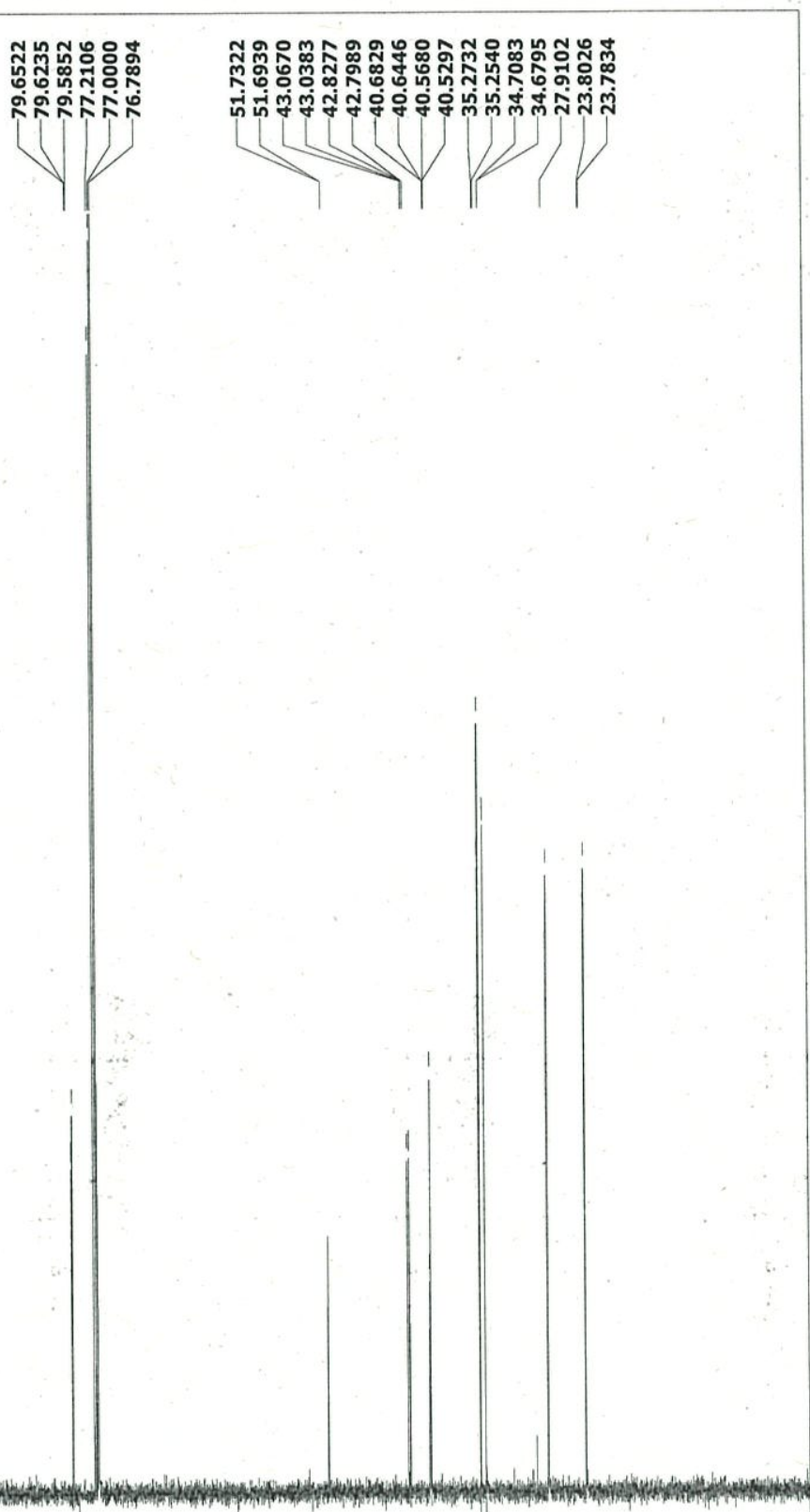

20

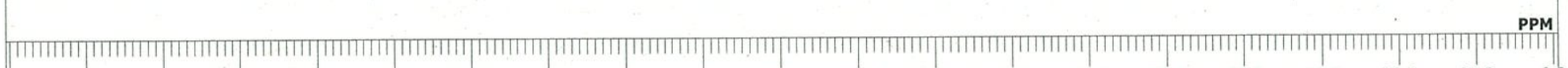

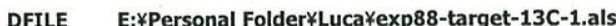

(1)

DATIM 07-03-2020 03:07:55

EXMOD single_pulse_dec

OBFRQ $\quad 150.92 \mathrm{MHz}$

$\begin{array}{ll}\text { OBSET } & 8.52 \mathrm{KHz} \\ \text { OBFIN } & 1.74 \mathrm{~Hz}\end{array}$

POINT 26214

FREQU $\quad 37878.21 \mathrm{~Hz}$

SCANS $\quad 307$

PD $\quad 0.6921 \mathrm{sec}$

PW1 $\quad 4.02 \mathrm{usec}$

IRNUC $1 \mathrm{H}$

CTEMP $24.2 \mathrm{C}$

EXREF $77.00 \mathrm{ppm}$

$\begin{array}{lc}\text { EXREF } & 77.00 \mathrm{ppm} \\ \text { BF } & 0.12 \mathrm{~Hz}\end{array}$

$13 \mathrm{C}$-NMR (CDCI3) ठ:

$79.62(\mathrm{t}, \mathrm{OH}, \mathrm{J}=5.1 \mathrm{~Hz})$,

(2.21 $(\mathrm{d}, \mathrm{H}, \mathrm{H}=5.8 \mathrm{~Hz})$,

$40.61(\mathrm{dd}, \mathrm{OH}, \mathrm{J}=17.3,5.8 \mathrm{~Hz})$

$35.26(\mathrm{~d}, \mathrm{OH}, \mathrm{J}=2.9 \mathrm{~Hz})$,
$34.69(\mathrm{~d}, \mathrm{OH}, \mathrm{J}=4.3 \mathrm{~Hz}$ ), 
E:ҰPersonal Folder¥Lucałexp88-target-31P-1.als

single pulse decoupled gated NOE

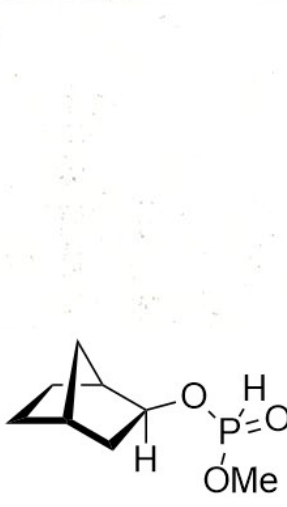

$3 e$ in $\mathrm{CDCl}_{3}$
DFILE E:ҰPersonal Folder¥Lucałexp88-target-31P-1.als

single pulse decoupled gated NO

OBNUC $31 \mathrm{P}$

EXMOD single_pulse_dec

OBFRQ $\quad 242.95 \mathrm{MHz}$

OBSET $\quad 4.04 \mathrm{KHZ}$

OBFINT $\quad 26214$

FREQU $172411.16 \mathrm{~Hz}$

SCANS 159

$\quad 0.1520 \mathrm{sec}$

$\begin{array}{lr}\text { PW1 } & 2.0000 \mathrm{sec} \\ \text { PW1 } & 4.00 \text { usec }\end{array}$

IRNUC 1H

CTEMP
SLVNT CDCL3.9

$\begin{array}{ll}\text { SLVNT } & \text { CDCL3 } \\ \text { EXREF } & 0.00 \mathrm{ppm}\end{array}$

$\begin{array}{ll}\text { BF } & 0.12 \mathrm{~Hz}\end{array}$

$31 \mathrm{P}$-NMR (CDCI3) $\delta$

$8.20(s, \mathrm{OH})$ 
E:₹Personal Folder¥Lucałexp85-target-1.als

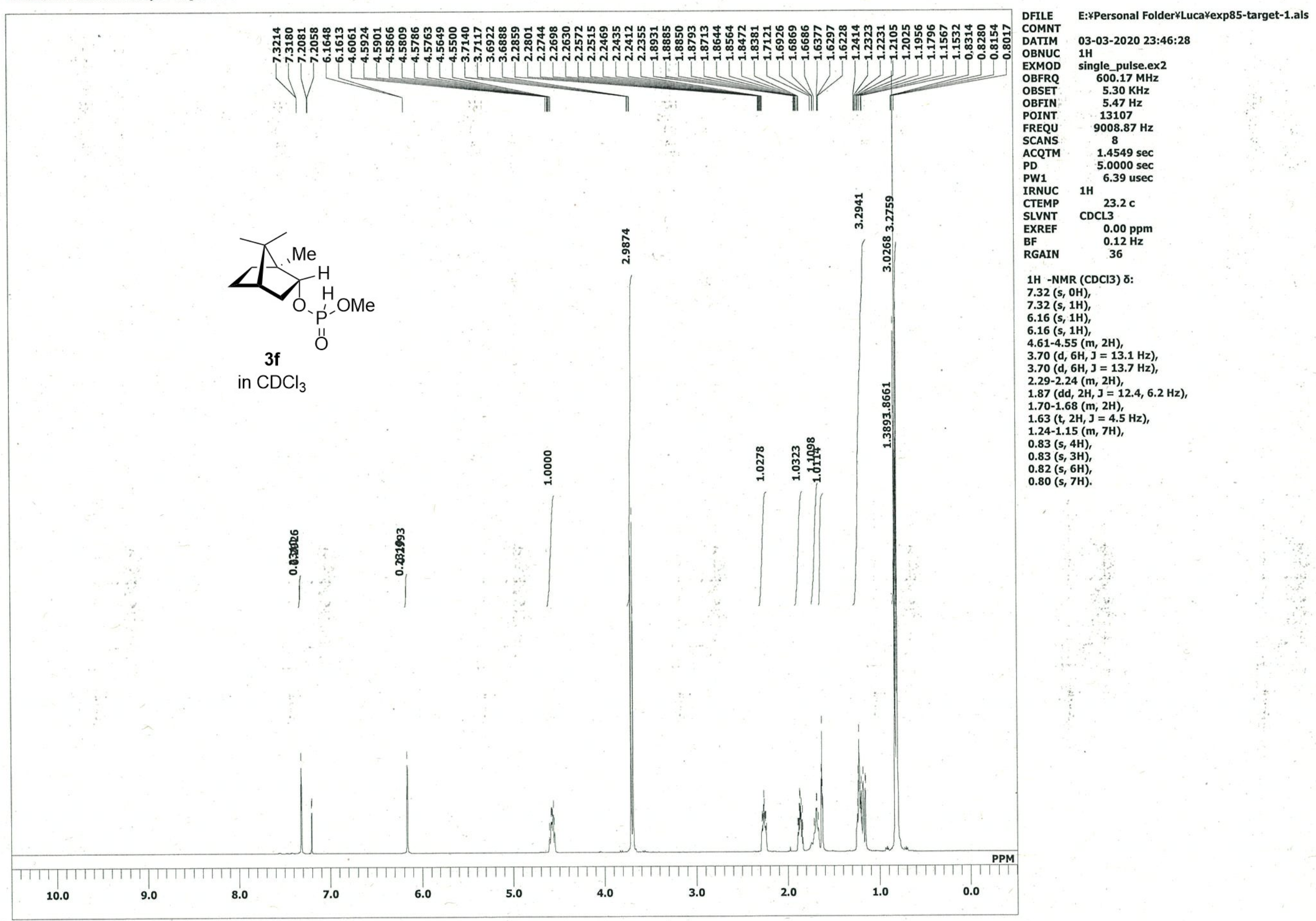



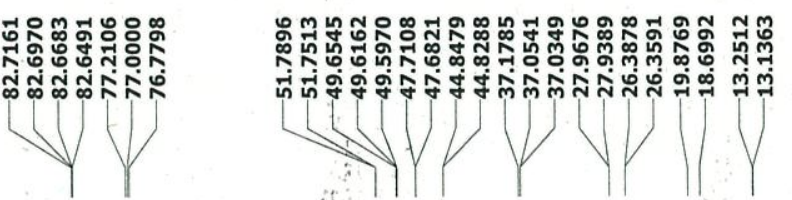

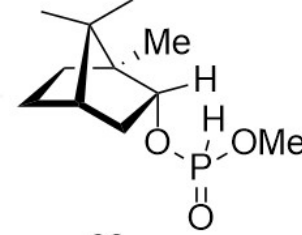

in $\mathrm{CDCl}_{3}$
DFILE E:¥Personal Folder*Lucałexp85-target-13C-1.als

COMNT single pulse decoupled gated NOE

4-03-2020 00:09:33

OBNUC $13 \mathrm{C}$

EXMOD single_pulse_dec

OBFRQ $\quad 150.92 \mathrm{MHz}$

OBSET $\quad 8.52 \mathrm{KHz}$

POINT 26214

FREQU $\quad 37878.21 \mathrm{~Hz}$

SCANS 366 .

$0.6921 \mathrm{sec}$

$\begin{array}{ll}\text { PD1 } & 2.0000 \mathrm{sec} \\ \text { PW1 } & 4.02 \text { usec }\end{array}$

IRNUC 1H

CTEMP $24.3 \mathrm{C}$

SLVNT CDCL3

$\begin{array}{ll}\text { EXREF } & 77.00 \mathrm{ppm} \\ \text { BF } & 0.12 \mathrm{~Hz}\end{array}$

$13 \mathrm{C}-\mathrm{NMR}(\mathrm{CDCl}) \mathrm{8}$

$82.68(\mathrm{q}, \mathrm{OH}, \mathrm{J}=3.4 \mathrm{~Hz})$,

$51.77(\mathrm{~d}, \mathrm{OH}, \mathrm{J}=5.8 \mathrm{~Hz})$,

$47.70(\mathrm{~d}, \mathrm{OH}, \mathrm{J}=4.3 \mathrm{~Hz})$

$44.84(\mathrm{~d}, \mathrm{OH}, \mathrm{J}=2.9 \mathrm{~Hz})$

$37.11(\mathrm{t}, \mathrm{OH}, \mathrm{J}=10.8 \mathrm{~Hz})$

$27.95(\mathrm{~d}, \mathrm{OH}, \mathrm{J}=4.3 \mathrm{~Hz})$

$19.88(\mathrm{~s}, \mathrm{OH})$
$18.70(\mathrm{~s}, \mathrm{OH})$

$13.19(\mathrm{~d}, 0 \mathrm{H}, \mathrm{J}=17.3 \mathrm{~Hz})$. 
XMOD single_pulse_dec

OBFRQ $242.95 \mathrm{MHz}$

$4.04 \mathrm{KHz}$

OBFIN $\quad 1.25 \mathrm{~Hz}$

FREQU $172411.16 \mathrm{~Hz}$

SCANS 116

ACQTM $\quad 0.1520 \mathrm{sec}$

$\begin{array}{ll}\text { PD } & 2.0000 \mathrm{sec} \\ \text { PW1 } & 4.00 \mathrm{sec}\end{array}$

IRNUC 1H $23.8 \mathrm{c}$

CTEMP
SLVNT CDCL3

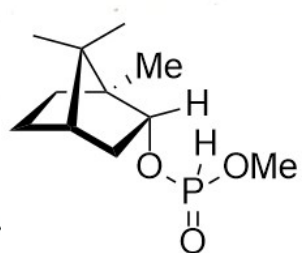

$0.00 \mathrm{ppm}$

$\begin{array}{lll}\text { BF } & 0.12 \mathrm{H} \\ \text { RGAIN } & 50\end{array}$

$31 \mathrm{P}-\mathrm{NMR}(\mathrm{CDCl} 3) \mathrm{\delta}$ :

$9.80(\mathrm{~d}, \mathrm{OH}, \mathrm{J}=39.5 \mathrm{~Hz})$

in $\mathrm{CDCl}_{3}$ 


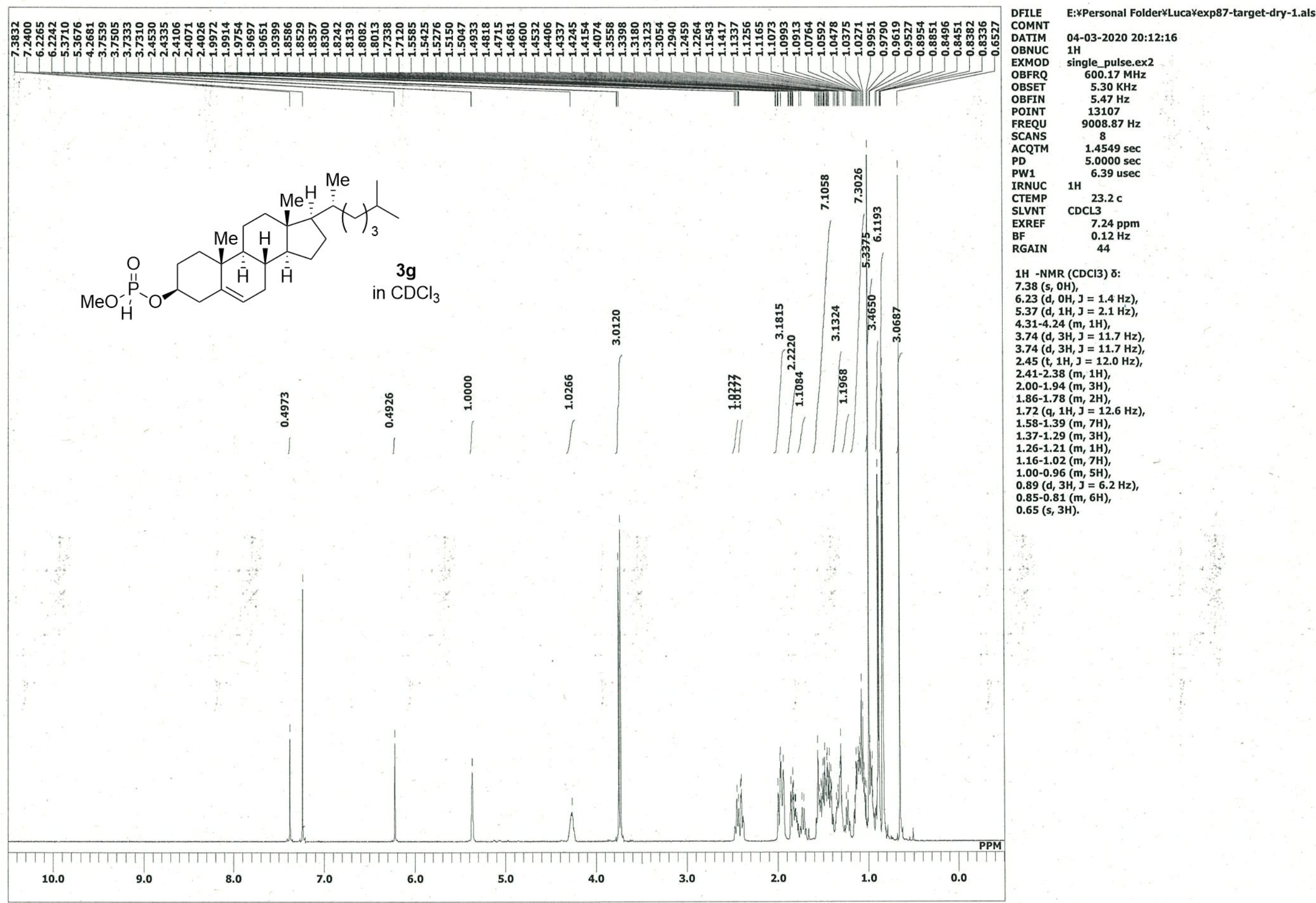


E:*Personal Folder¥Luca¥exp87-target-conc3-13C-1.als

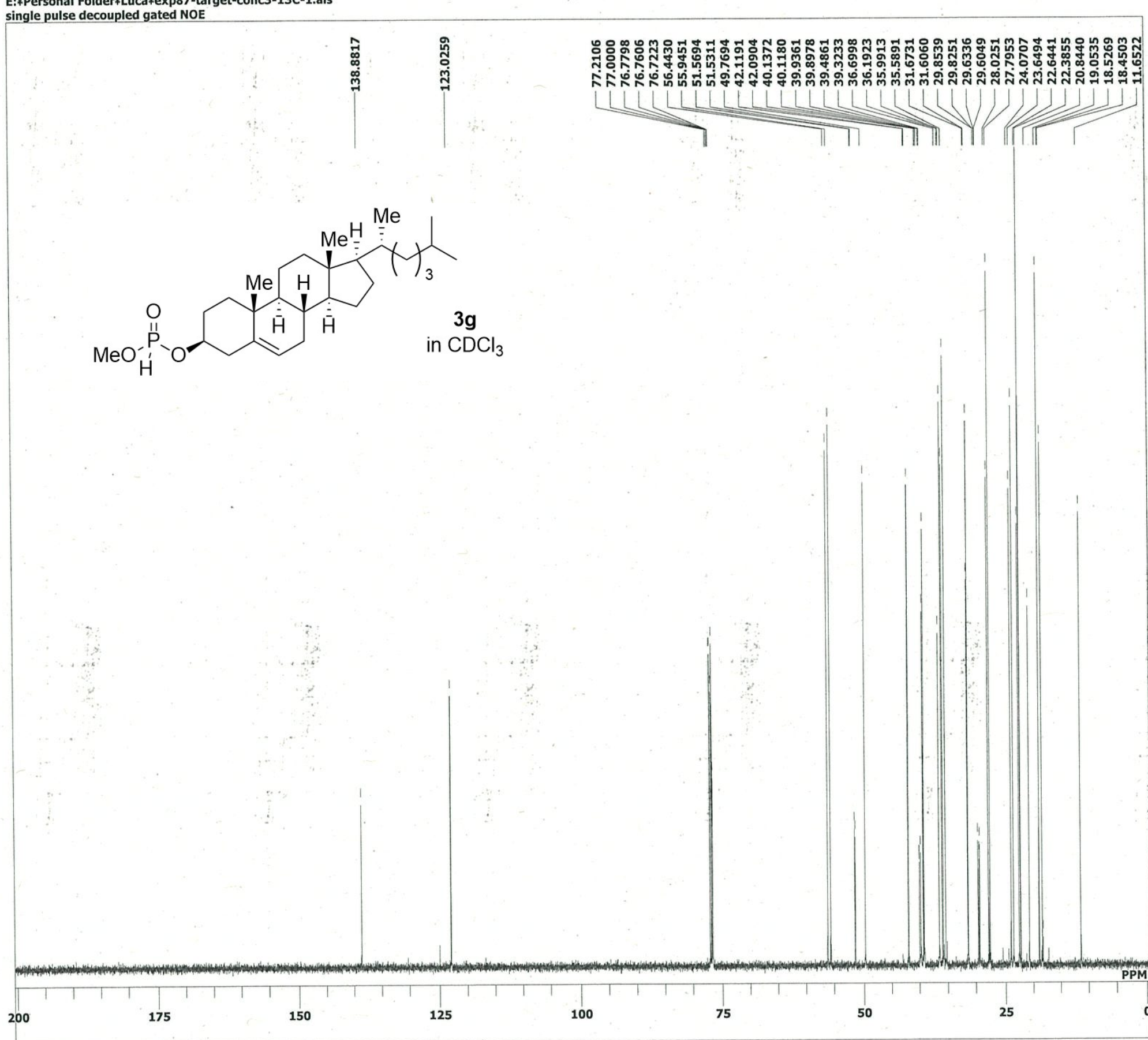

DFYPersonal FolderæLucałexp87-target-conc3-13C-1.als

(

XMOD single_pulse_dec

OBFRQ $\quad 150.92 \mathrm{MHz}$

$\begin{array}{ll}\text { OBSET } & 8.52 \mathrm{KHz} \\ \text { OBFIN } & 1.74 \mathrm{~Hz}\end{array}$

POINT 26214

FREQU $37878.21 \mathrm{~Hz}$

SCANS

$0.6921 \mathrm{sec}$

PW1 4.02 usec

IRNUC

CTEMP

SLVNT CDCL3 3

$\begin{array}{ll}\text { EXREF } & 77.00 \mathrm{ppm} \\ \text { BF } & 0.12 \mathrm{~Hz}\end{array}$ 
E:ҰPersonal FolderҰLuca¥exp87-target-31P-1.als

single pulse decoupled gated NOE

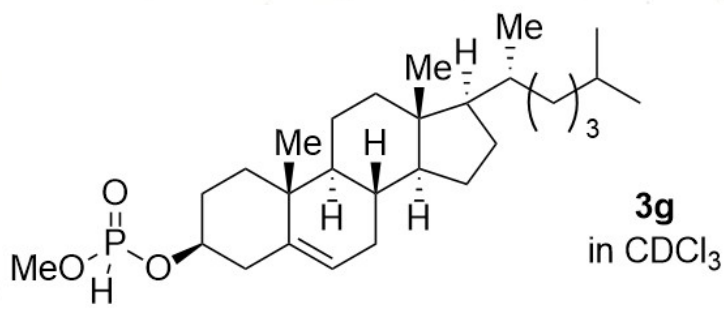

EFILE

Secoupled gated NOE

DATIM 04-03-2020 07:56:58

EXMOD single_pulse_dec

OBFRQ $242.95 \mathrm{MHz}$

OBFIN $\quad 1.25 \mathrm{~Hz}$

POINT 26214

FREQU $\quad 172411.16 \mathrm{~Hz}$

296

$0.1520 \mathrm{sec}$
ACQTM

PW1 $4.00 \mathrm{usec}$

$\begin{array}{ll}\text { IRNUC } & \text { 1H } \\ \text { CTEMP } & 24.1 \mathrm{c}\end{array}$

CTEMP
SLVNT $24.1 \mathrm{C}$

$\begin{array}{ll}\text { SLVNT } & \text { CDCL } 3 \\ \text { EXREF } & 0.00 \mathrm{ppm}\end{array}$

$\begin{array}{lll}\text { BF } & 0.12 \mathrm{~Hz}\end{array}$

31P -NMR (CDCl3) 8

$8.15(\mathrm{~s}, \mathrm{OH})$ 


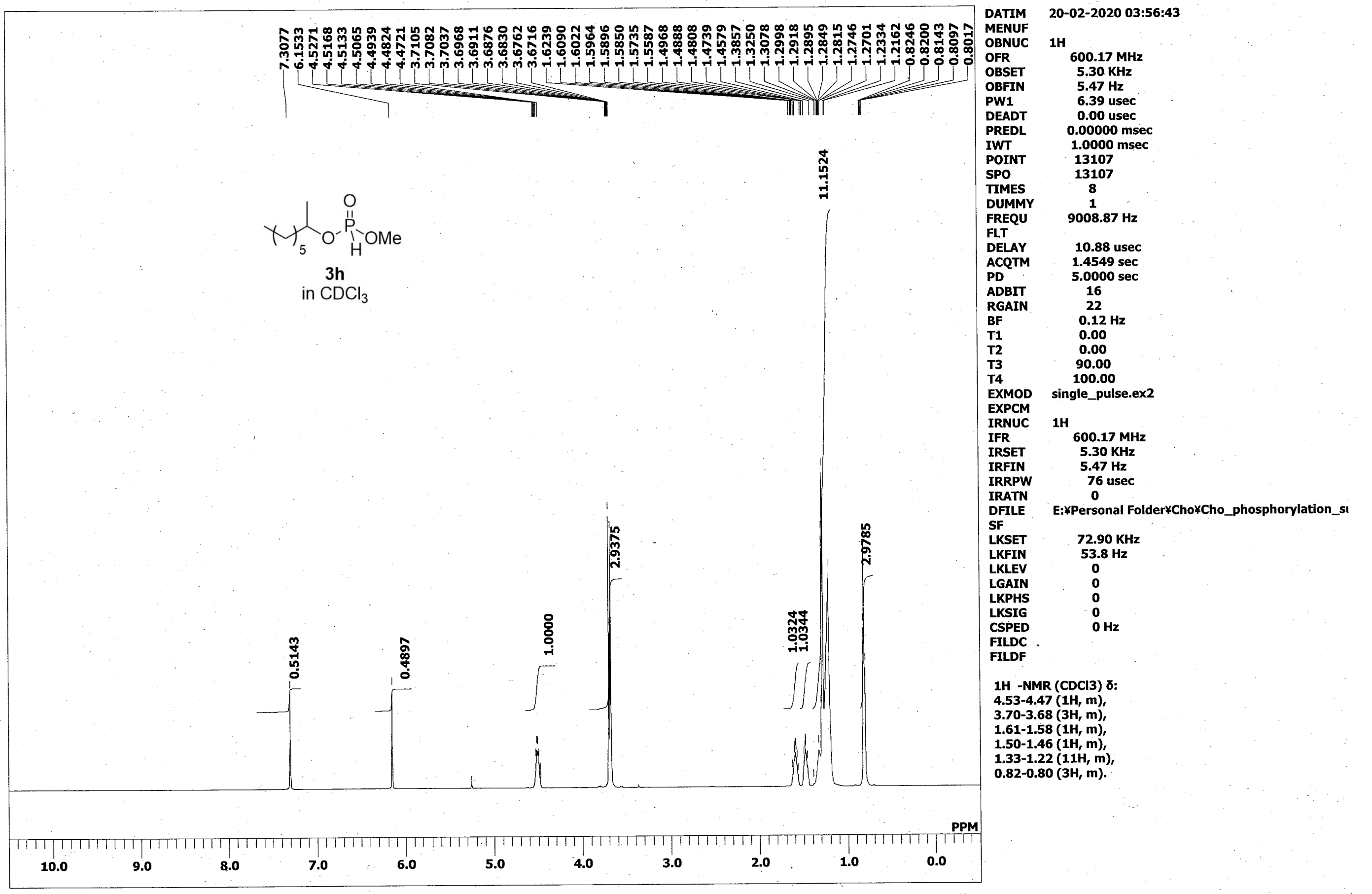



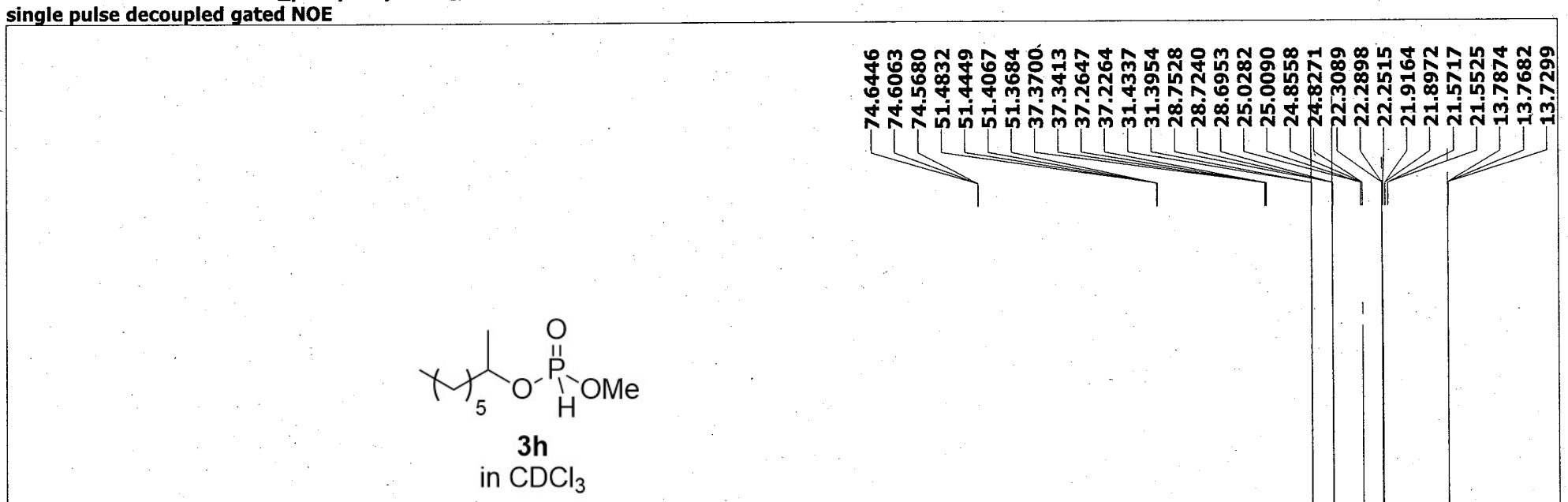

DATIM

20-02-2020 03:58:57

MENUF

in $\mathrm{CDCl}_{3}$

OBNUC

OFR

$13 \mathrm{C}$

OBFIN

PWFI

DEADT

PREDL

IWT

POINT

SPO

TIMES

FREQU

DELAY

ACQTM

PD

ADBIT

RGAIN

BF

T1

T3

0.00
90.00
100.00

$150.92 \mathrm{MHz}$ $8.52 \mathrm{KHz}$
$1.74 \mathrm{~Hz}$

$1.74 \mathrm{~Hz}$
4.02 usec

4.02 usec
0.00 usec

$0.00 \mathrm{usec}$
$0.00000 \mathrm{msec}$

$0.00000 \mathrm{msec}$

26214

26214

35

37878.21 Hz

21.12 usec

$0.6921 \mathrm{sec}$

$0 \mathrm{sec}$

16

$0.12 \mathrm{~Hz}$

0.00

EXMOD single_pulse_dec

EXPCM

IRNUC

$1 \mathrm{H}$.

$\begin{array}{ll}\text { IRSET } & 5.30 \mathrm{KHz} \\ \text { IRFIN } & 5.47 \mathrm{~Hz}\end{array}$

$600.17 \mathrm{MHz}$

$\begin{array}{ll}\text { IRFIN } & 5.47 \mathrm{~Hz} \\ \text { IRRPW } & 76 \mathrm{usec}\end{array}$

IRRPW 76

DFILE E:*Personal Folder*Cho*Cho_phosphorylation_s

DFI

E:*Personal Fo

LKSET $\quad \mathbf{7 2 . 9 0} \mathrm{KHz}$

LKFIN $\quad 53.8 \mathrm{~Hz}$

$\begin{array}{ll}\text { LKLEV } & 0 \\ \text { LGAIN } & 0\end{array}$

LGAIN

LKSIG

0

CSPED

0
0
0

FILDC

$13 \mathrm{C}$-NMR ( $\mathrm{CDCl} 3) \delta$ :

74.63-74.59 (OH, $\mathrm{m})$,

$51.48(\mathrm{OH}, \mathrm{s})$,

$\mathbf{5 1 . 4 4}(\mathrm{OH}, \mathrm{s})$,

$51.41(\mathrm{OH}, \mathrm{s})$,

$51.37(\mathrm{OH}, \mathrm{s})$,

$37.37(\mathrm{OH}, \mathrm{s})$,

$37.34(\mathrm{OH}, \mathrm{s})$,

$37.26(\mathrm{OH}, \mathrm{s})$,

$37.23(\mathrm{OH}, \mathrm{s})$,

PPM $31.43\left(\mathrm{OH}_{\mathrm{s}} \mathrm{s}\right)$

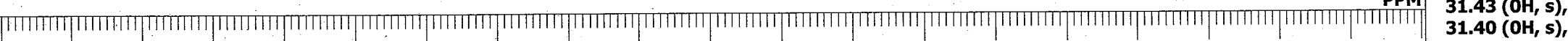

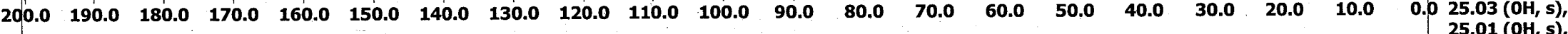

$25.01(\mathrm{OH}, \mathrm{s})$,

$24.86(\mathrm{OH}, \mathrm{s})$,

$24.83(\mathrm{OH}, \mathrm{s})$,

$22.31(\mathrm{OH}, \mathrm{s})$ 
E:*Personal Folder*Cho*Cho_phosphorylation_sub*sub3e_phos.als single pulse decoupled gated NOE

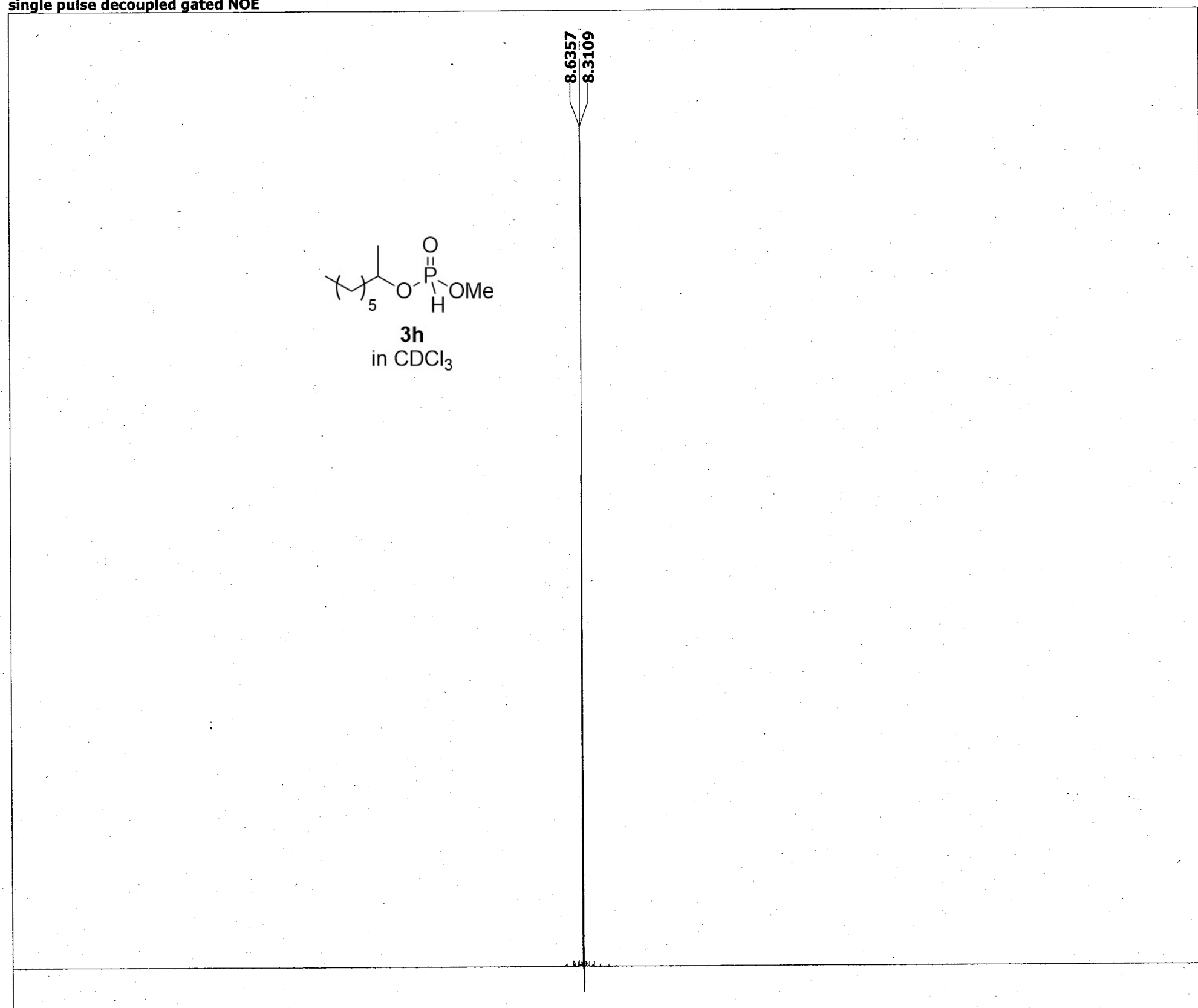

DATIM

OBNUC

OFR

PEAT

DEADT

POINT

SPO

TIMES

DUMMY

FREQU

FLT

DELAY

ACQTM
PD

ADBIT

RGAIN

BF

T2

T3

EXMOD

EXPCM

IRNUC

IFR
IRSET

IRFIN

IRRPW

IRATN

DFI

LKSE

LKFIN

LKLEV

LGAIN

LKPHS

LKSIG

CSPED

FILDC

FILDC
242.95 MHz $4.04 \mathrm{KHz}$

4.00 usec

0.00 usec

0.0000 usec

$10000 \mathrm{msec}$

$1.0000 \mathrm{msec}$

26214
47

4

$172411.16 \mathrm{~Hz}$

20.50 usec

$0.1520 \mathrm{sec}$

$2.0000 \mathrm{sec}$

16

$0.12 \mathrm{~Hz}$

0.00

0.00

000

single pulse dec

$1 \mathrm{H}$

$600.17 \mathrm{MHz}$

$5.30 \mathrm{KHz}$

$5.47 \mathrm{~Hz}$

76 usec

rsonal Folder¥Cho¥Cho_phosphorylation_si

$0.00 \mathrm{KHz}$

$0.0 \mathrm{~Hz}$

0

0

$\mathrm{OHz}$ 


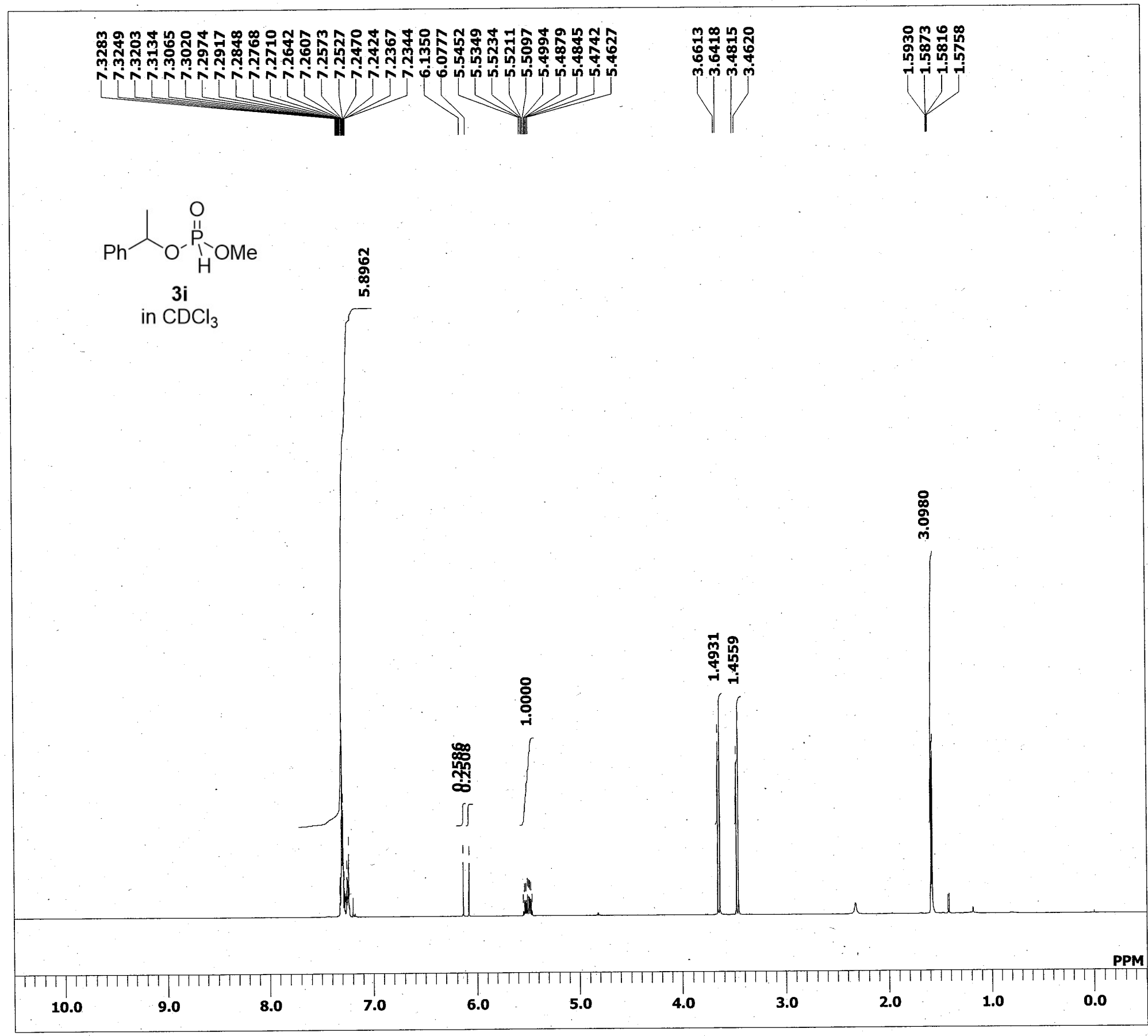

DATIM 25-07-2019 15:25:49

MENUF

BNUC $1 \mathrm{H}$

$\quad 600.17 \mathrm{MHz}$

OBSET $\quad 5.30 \mathrm{KHz}$

$5.47 \mathrm{~Hz}$

$\quad 6.39$ usec

DEFI $\quad 0.00$ usec

IWT $\quad 1.0000 \mathrm{msec}$

$1.0000 \mathrm{msec}$
$\mathrm{P}$

SPO 13107

TIMES $\quad 8$

DUMMY 11

FREQU $\quad 9008.87 \mathrm{~Hz}$

FLT $\quad 10.88$ usec

ACQTM $\quad 10.88$ usec

PD $\quad 5.0000 \mathrm{sec}$

ADBIT 16

RGAIN $\quad 36$

BF $\quad 0.12 \mathrm{~Hz}$

$\begin{array}{ll}11 & 0.00 \\ 72 & 0.00\end{array}$

T3 90.00

T4 100.00

EXMOD single pulse.ex2

$\begin{array}{ll}\text { EXPCM } & \text { single_pulse.ex } \\ \text { IRNUC } & \text { 1H }\end{array}$

IFR $\quad 600.17 \mathrm{MHz}$

IRSET $\quad 5.30 \mathrm{KHz}$

IRFIN $\quad 5.47 \mathrm{~Hz}$

IRRPW $\quad 76$ usec

IRATN 0

DFILE E:*Personal Folder*Cho*Cho_phosphorylation_si

:Fersonal Fo

$\quad 72.90 \mathrm{KHz}$

LKFIN $\quad 53.8 \mathrm{~Hz}$

$\begin{array}{ll}\text { LKLEV } & 0 \\ \text { LGAIN } & 0\end{array}$

LGAIN

KSIG

CSPED

FILDC

FILDF

1H -NMR ( $\mathrm{CDCl} 3) \delta$ :

7.33-7.23 $(6 \mathrm{H}, \mathrm{m})$,

5.55-5.46 (1H, m),

$3.65(1 \mathrm{H}, \mathrm{d}, \mathrm{J}=11.68 \mathrm{~Hz})$

$3.47(1 \mathrm{H}, \mathrm{d}, \mathrm{J}=11.68 \mathrm{~Hz})$

$1.58(3 \mathrm{H}, \mathrm{q}, \mathrm{J}=3.44 \mathrm{~Hz})$. 


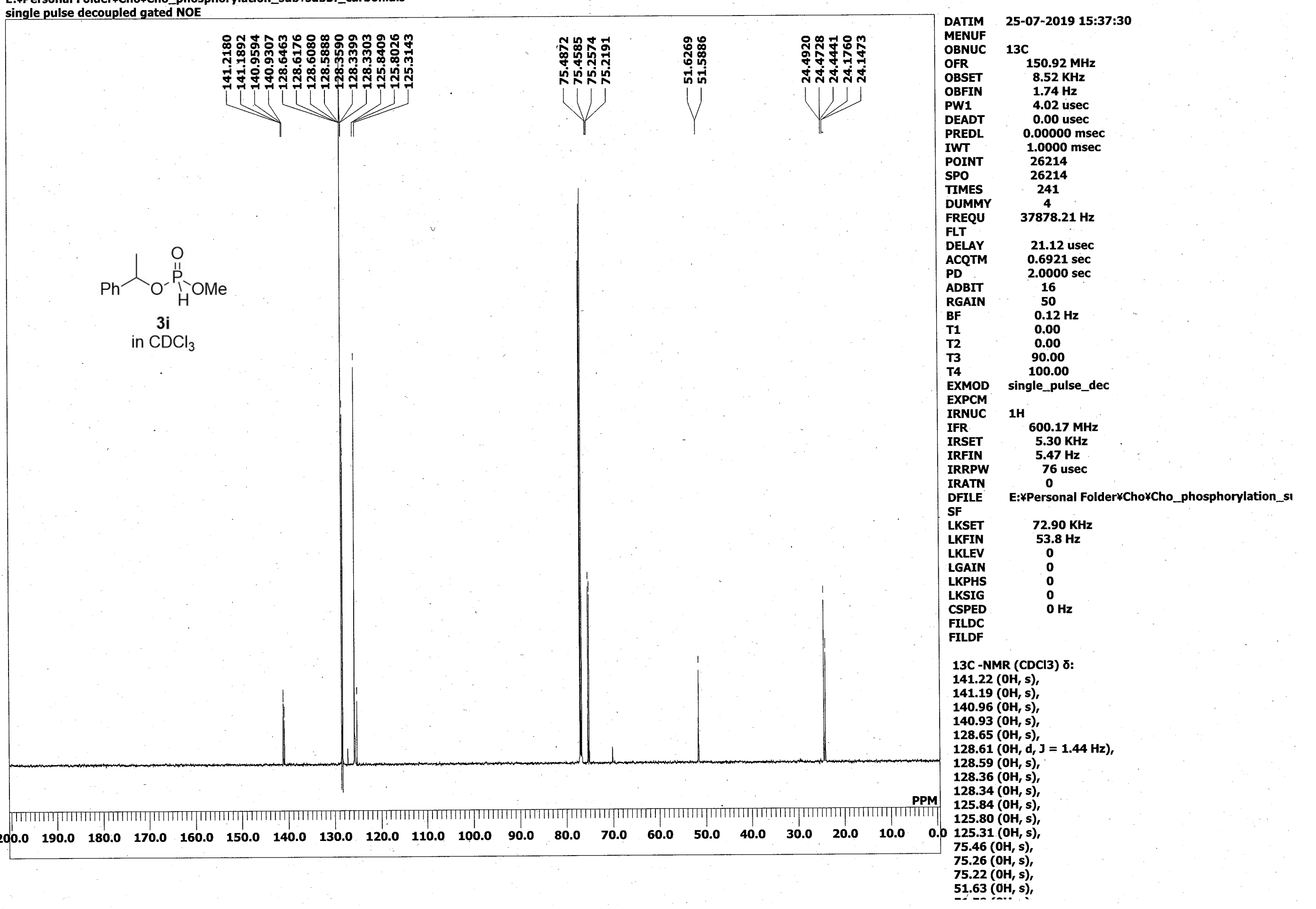




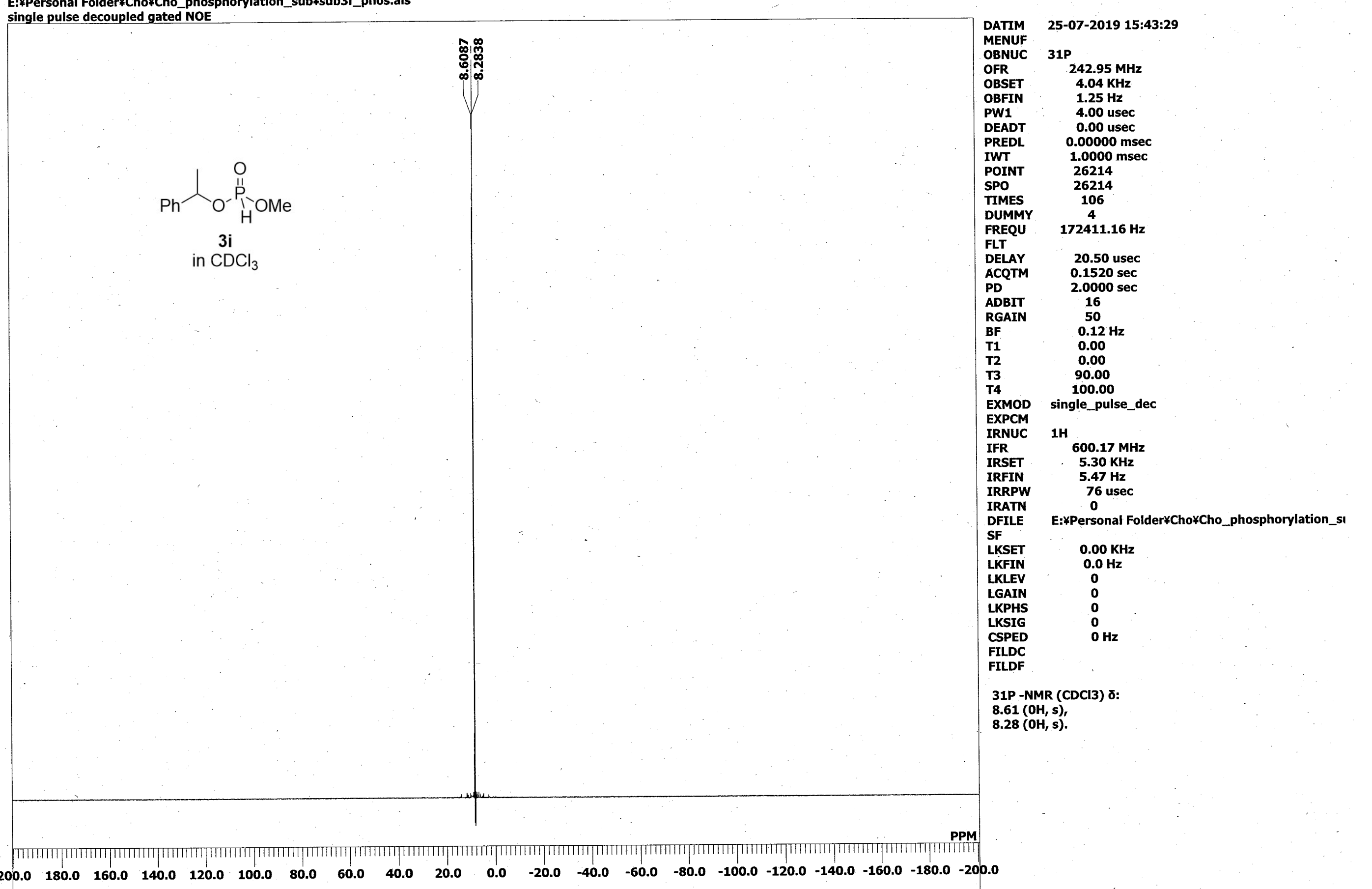




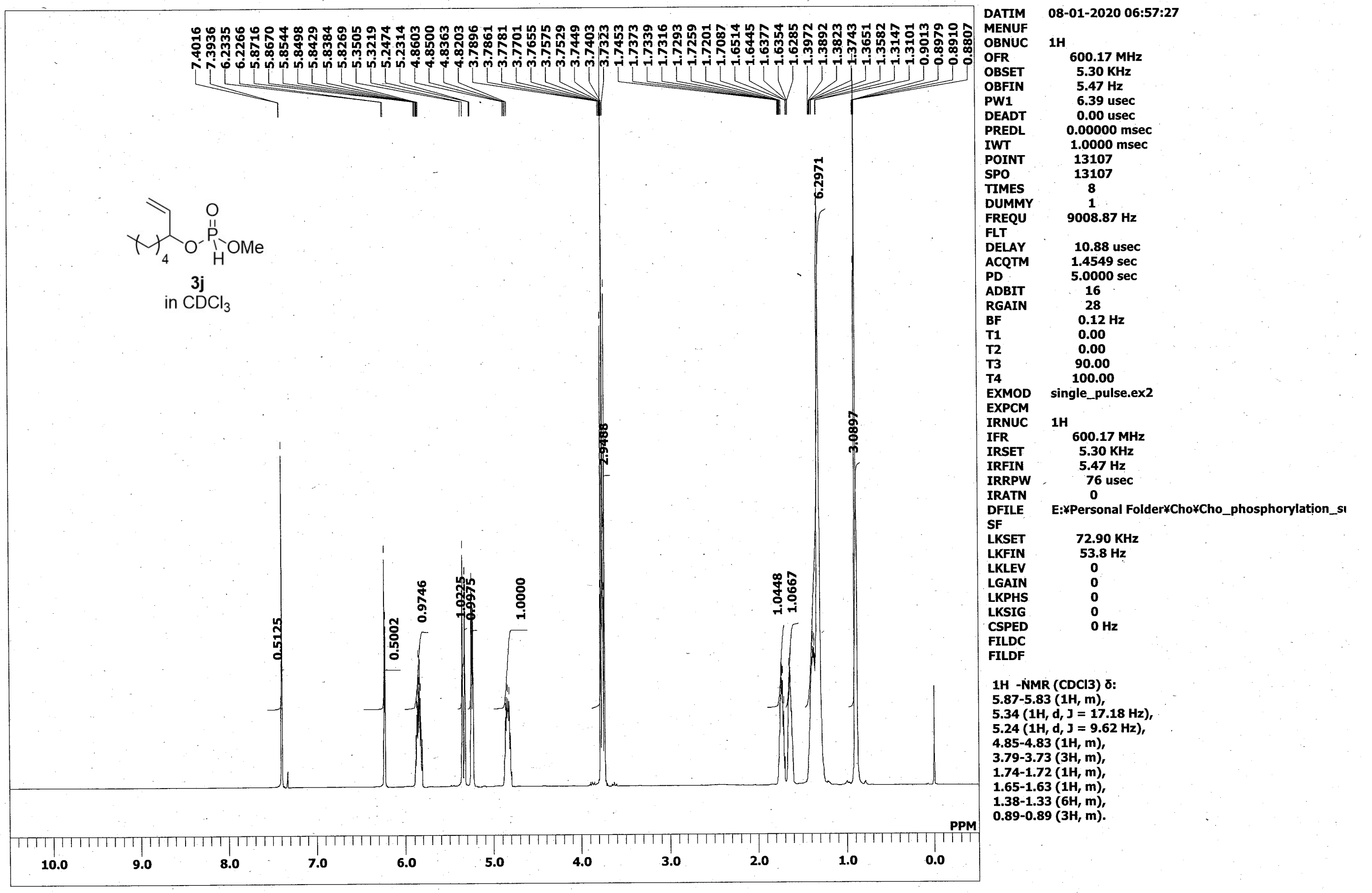


E:*Personal Folder*Cho\#Cho_phosphorylation_sub*sub3g_carbon.als single pulse decoupled gated NOE

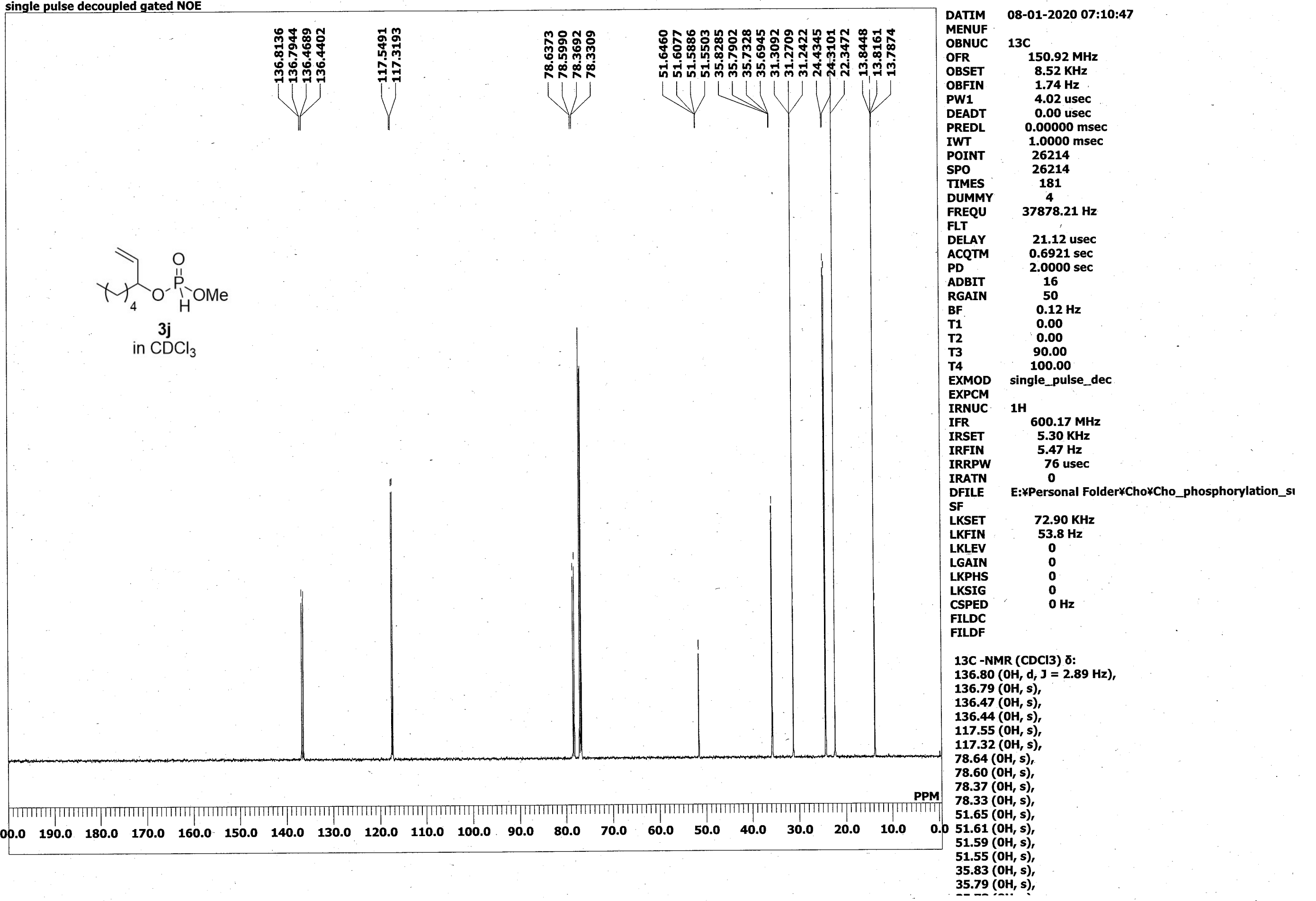




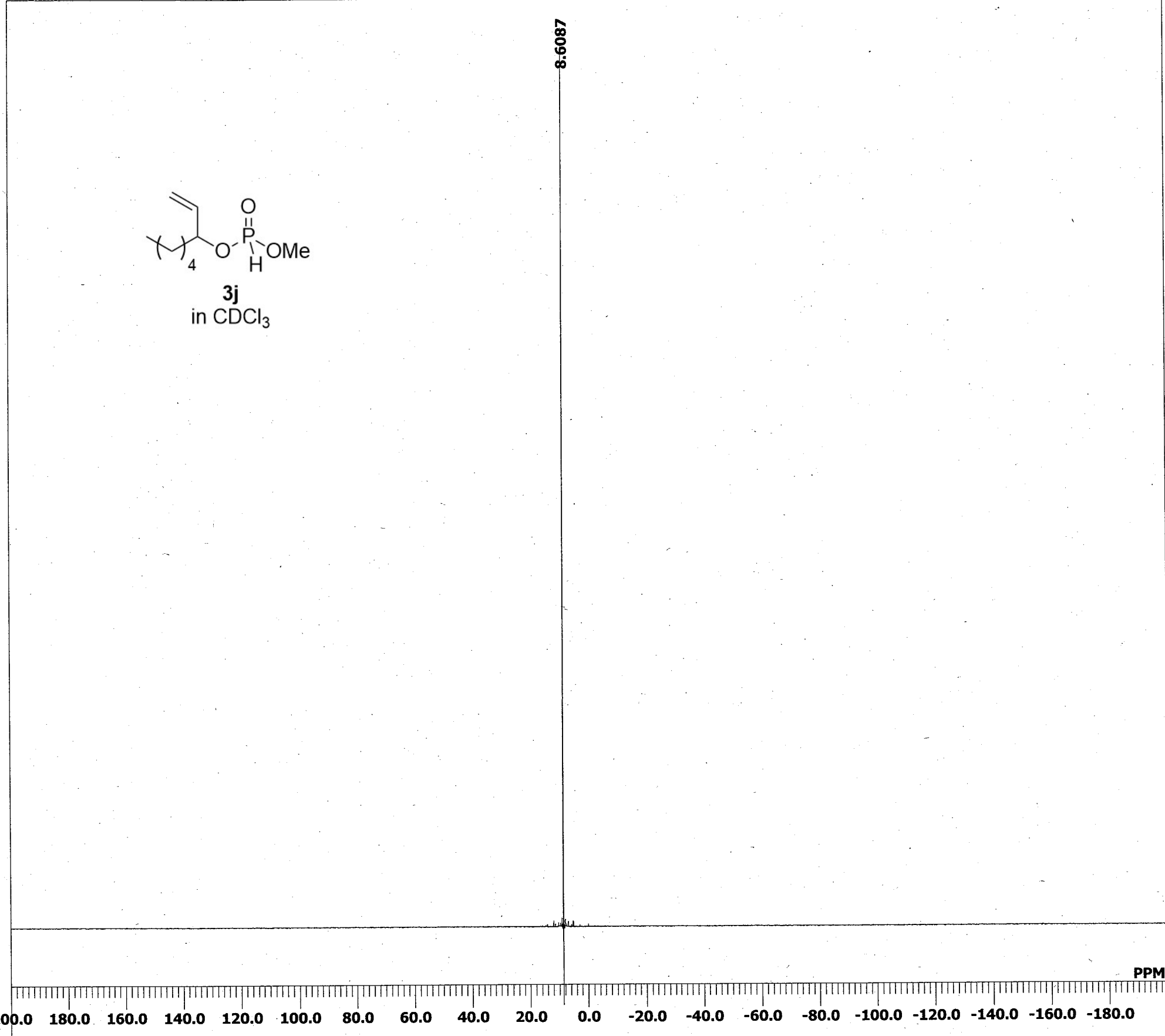

_sub*sub3g_phos.als

DATIM

MENUF

OBNUC

OFR

OBSET

OBFIN

PW1

DEADT

IWT

POINT

SPO

DUMMY

FREQU

FREQU

DELAY

ACQTM

PD

ADBIT

RGAIN

BF

T1

T3

T4

EXMOD

EXPCM

IRNUC

IFR

IRSET

IRRPW

IRATN

DF

LKSET

LKFIN

LKLEV

LGAIN

LKPHS

LKPHS

CSPED

CSPED

FILDF
-01-2020 07:00:50

$242.95 \mathrm{MHz}$

$4.04 \mathrm{KHz}$

$1.25 \mathrm{~Hz}$
$4.00 \mathrm{usec}$

4.00 usec

$0.0000 \mathrm{msec}$

$0.00000 \mathrm{msec}$
$1.0000 \mathrm{msec}$

26214

26214
41
4

$172411.16 \mathrm{~Hz}$

\subsection{0 usec}

$0.1520 \mathrm{sec}$

2.0000
16
50

$0.12 \mathrm{~Hz}$

0.00

0.00

90.00

single_pulse_dec

1H

$600.17 \mathrm{MHz}$

$5.30 \mathrm{KHz}$
$5.47 \mathrm{~Hz}$

76 usec

0

E:*Personal Folder*Cho*Cho_phosphorylation_si

$0.00 \mathrm{KHz}$

$0.0 \mathrm{~Hz}$

0

0

O Hz 


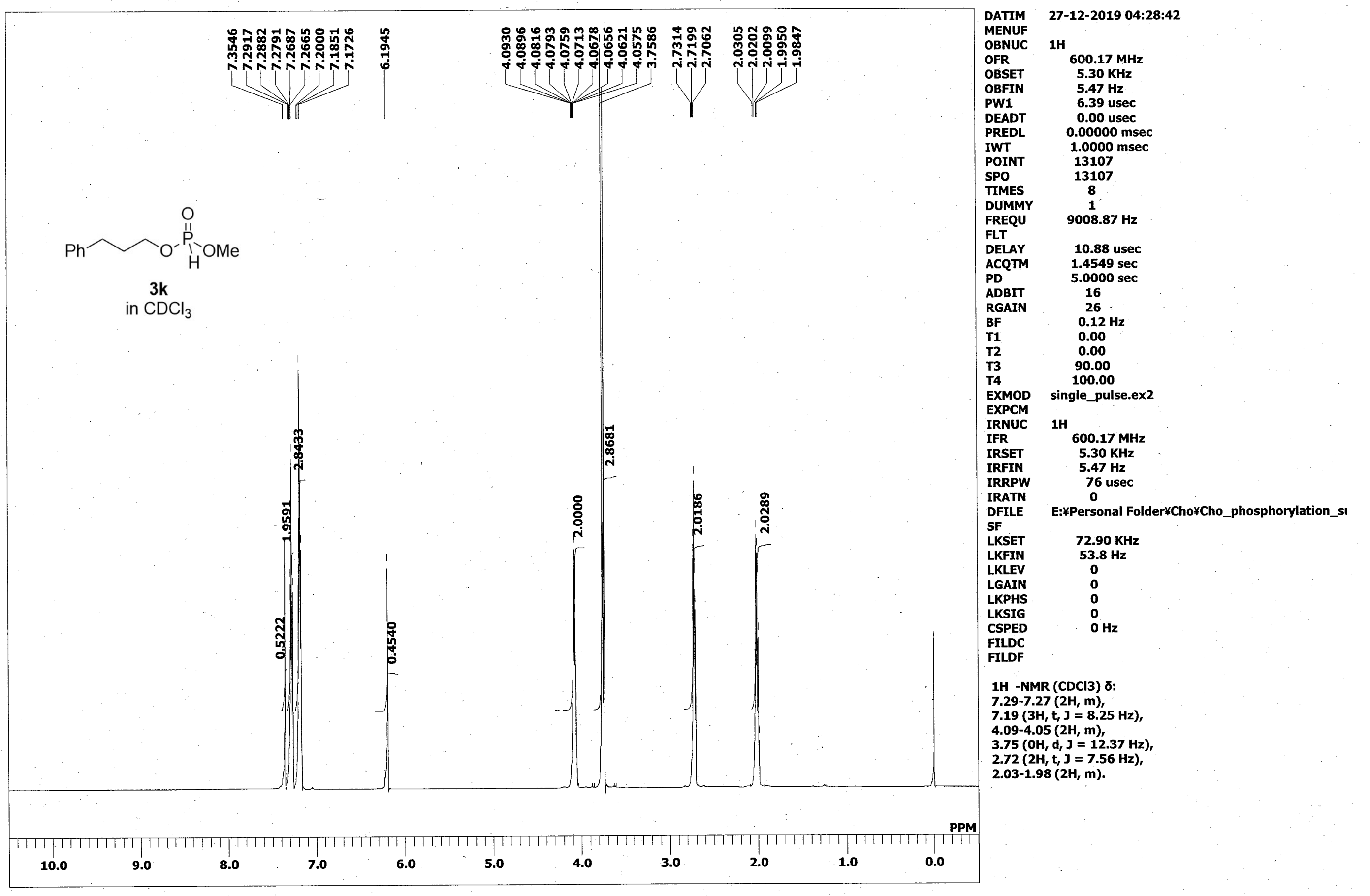


E:*Personal Folder*Cho\#Cho_phosphorylation_sub*sub3i_carbon.als single pulse decoupled gated NOE

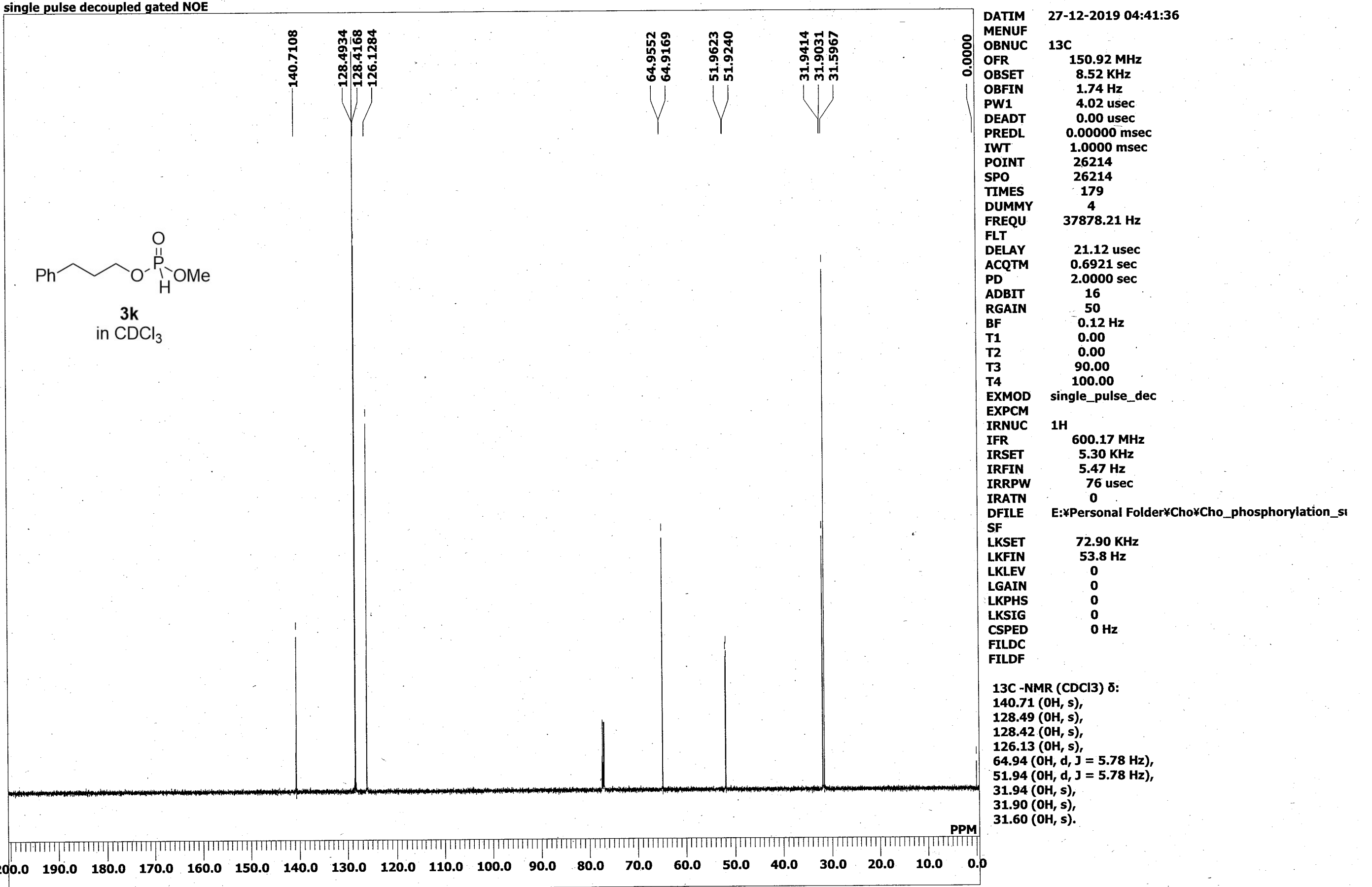


E:¥Personal Folder¥Cho¥Cho_phosphorylation_sub¥sub3i_phos.als

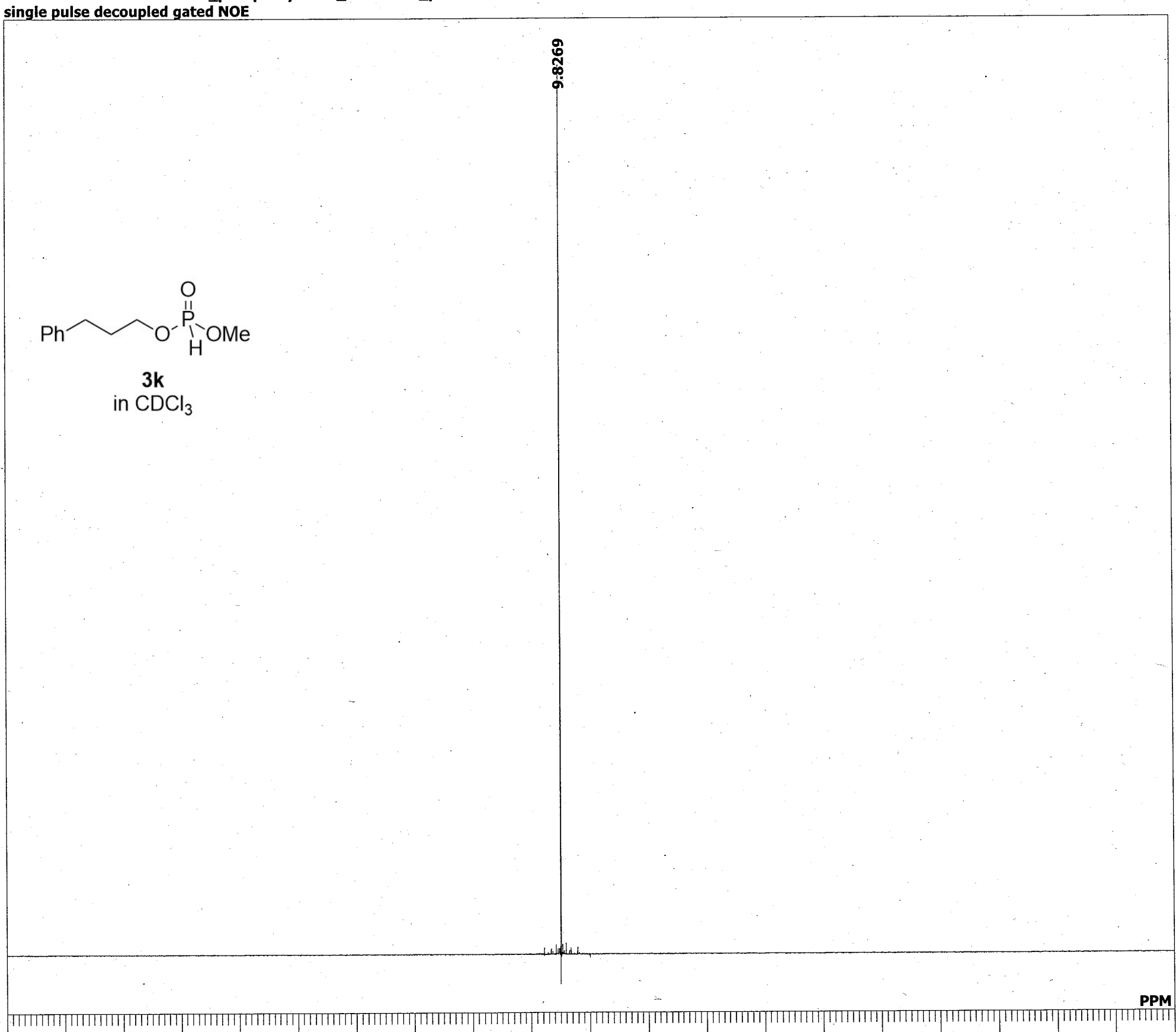

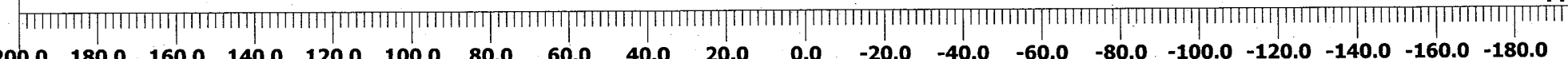

DATIM

31P

OBSET

OBFIN

PW1

DEADT

IWT

POINT

SPO

TIMES

DUMMY

FLT

DELAY

ACQTM

PD

ADBIT

RGAIN

BF

T2
T3
T4

T4

EXMOD

EXPCM

IRNUC

IFR

IRSET

IRFIN

IRRPW

DFILE

SF

LKSET

LKFIN

LKLEV

LGAIN

LKPHS

LKSIG

TILDC

ILDF

$-12-2019$ 04:31:40

$242.95 \mathrm{MHz}$

$4.04 \mathrm{KHz}$

4.00 usec

0.00 usec

$0.00000 \mathrm{msec}$

$1.0000 \mathrm{msec}$

$1.0000 \mathrm{~ms}$

26214

27

16

20.50 usec

$0.1520 \mathrm{sec}$

$2.0000 \mathrm{sec}$

16

50
0.12

$0.12 \mathrm{~Hz}$

0.00

90.00

100.00

1H

$600.17 \mathrm{MH}$

$5.30 \mathrm{~Hz}$
$.6 \mathrm{~Hz}$

76 usec

0

E:*Personal Folder*Cho\#Cho_phosphorylation_st

$0.00 \mathrm{KHz}$

0

0

$\mathrm{Hz}$ 


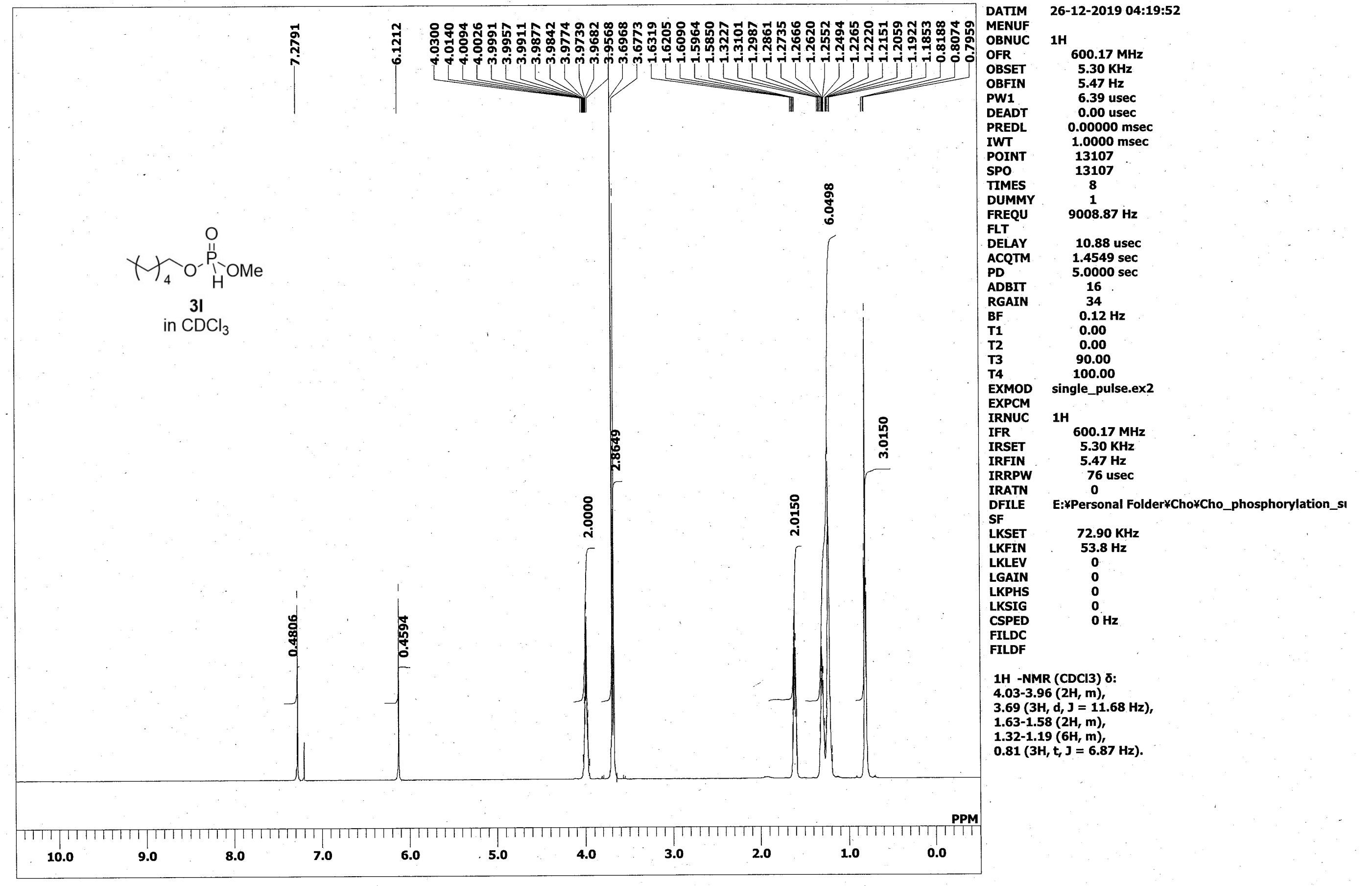



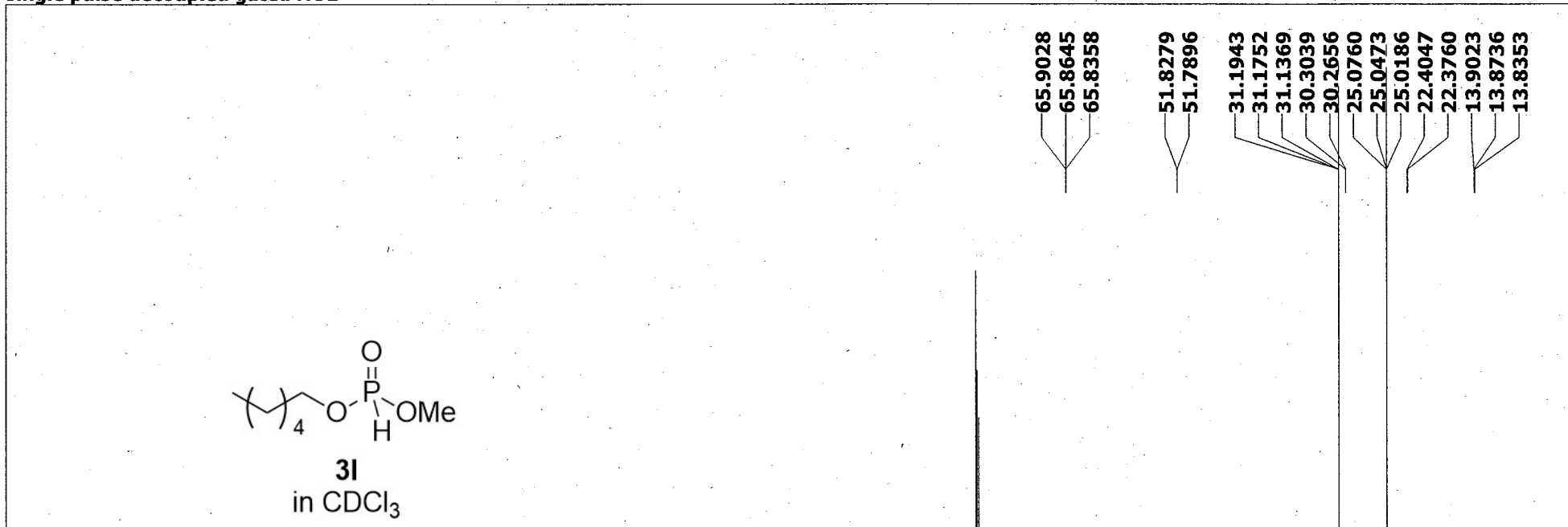

26-12-2019 04:30:57

MENUF

$13 \mathrm{C}$

OBSET

$150.92 \mathrm{MHz}$

$8.52 \mathrm{KHz}$

$1.74 \mathrm{~Hz}$

PW1 $\quad 4.02$ usec

DEADT $\quad 0.00$ usec

PREDL $\quad 0.00000 \mathrm{msec}$

$1.0000 \mathrm{msec}$

POINT 26214

TIMES $\quad 207$

TIMES $\quad 207$

$\begin{array}{lc}\text { FREQU } & \mathbf{4} \\ \text { FR } & 378.21 \mathrm{~Hz}\end{array}$

21.12 usec

$\begin{array}{ll}\text { DELAY } & 21.12 \mathrm{usec} \\ \text { ACQTM } & 0.6921 \mathrm{sec}\end{array}$

ADBIT

RGAIN

BF

16

$0.12 \mathrm{~Hz}$

T2 $\quad 0.00$

$\begin{array}{ll}T 3 & 90.00 \\ T 4 & 100.00\end{array}$

EXMOD single_pulse_dec

EXPCM

EXPCM

IRNUC

$1 \mathrm{H}$

IFR

$600.17 \mathrm{MHz}$

IRSET

IRFIN

$5.30 \mathrm{KHz}$

$\quad 56$ usec

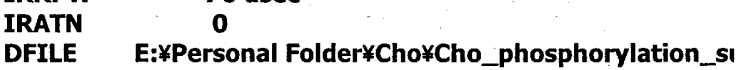

SF

E:¥Personal Folde

LKSET $\quad \mathbf{7 2 . 9 0} \mathrm{KHz}$

LKFIN $\quad 53.8 \mathrm{~Hz}$

LKLEV $\quad 0$

$\begin{array}{ll}\text { LGAIN } & 0 \\ \text { LKPHS } & 0 \\ \text { LKSIG } & 0\end{array}$

CSPED

FIIDC

FILDC

13C -NMR ( $\mathrm{CDCl} 3)$ ठ:

$65.88(\mathrm{OH}, d, J=5.78 \mathrm{~Hz})$,

$51.81(\mathrm{OH}, d, \mathrm{~J}=\mathbf{5 . 7 8 \mathrm { Hz }}$,

$31.18(\mathrm{OH}, \mathrm{s})$

$30.28(\mathrm{OH}, \mathrm{d}, \mathrm{J}=5.78 \mathrm{~Hz})$

$25.05(\mathrm{OH}, \mathrm{s})$

$25.05(\mathrm{OH}, \mathrm{s})$

$22.40(\mathrm{OH}, \mathrm{s})$
$13.87\left(\mathrm{OH}^{\mathrm{s}} \mathrm{s}\right)$ 
E:¥Personal Folder*Cho¥Cho_phosphorylation_sub¥sub3j_phos.als

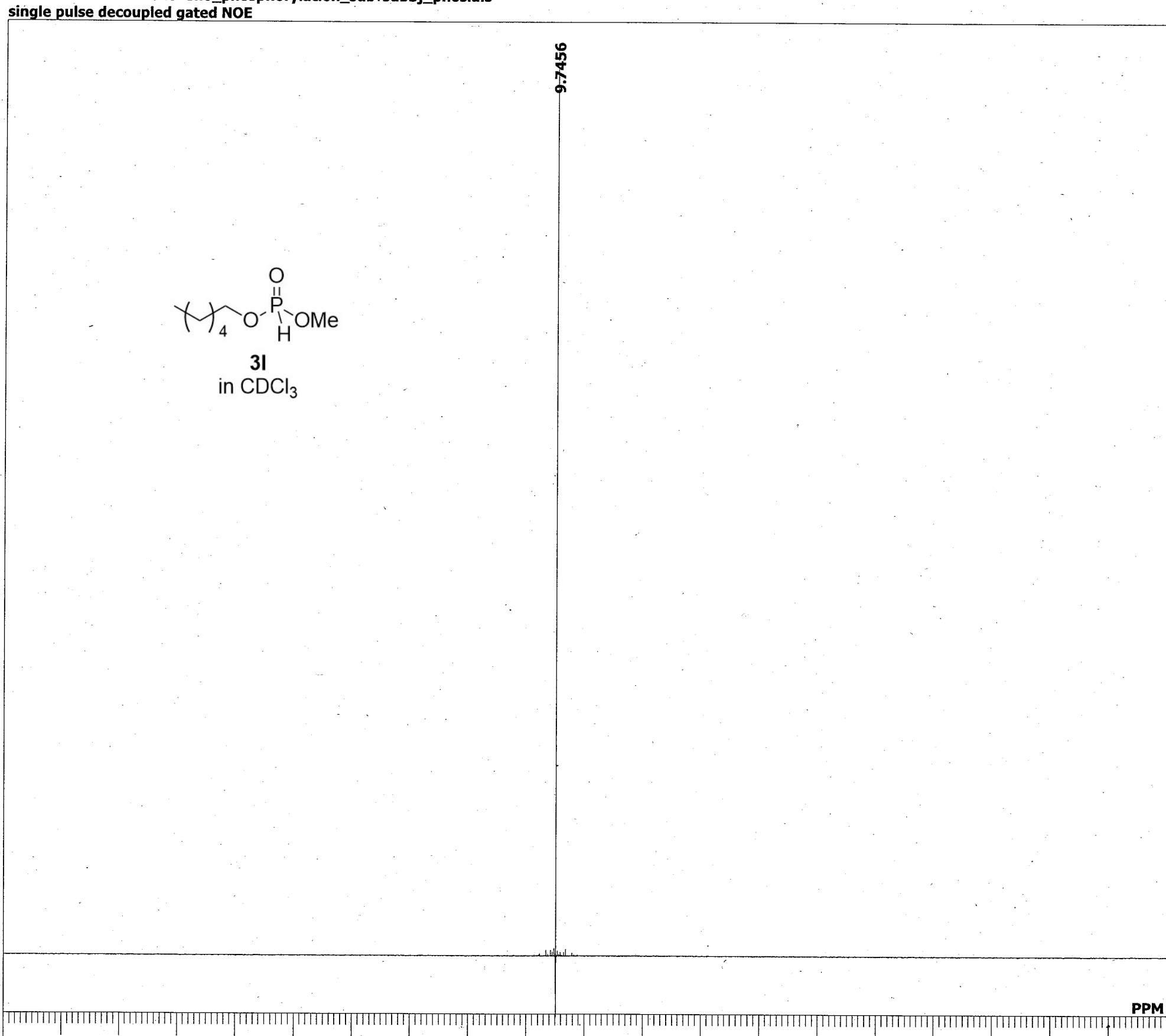

DATIM

MENUF

OBSET

OBFIN

PW1

DEADT

PEDL

POIN

SPO

TIMES

DUMMY

FLT

DELAY

PD

ADBIT

RGAIN

BF

T1

12

T4

EXMOD

EXPCM

IRNUC

IFR
IRSET

IRSET
IRFIN

IRFIN
IRRPW

IRATN

DFILE

KSET

LKFIN

KLEV

LGAIN

KPHS

(

CSPED

ILDC

FILDF
26-12-2019 04:35:01

$31 P$

242.95 MHz

$4.04 \mathrm{KHz}$

$1.25 \mathrm{~Hz}$

4.00 usec

0.00 usec

$0.00000 \mathrm{msec}$

$1.0000 \mathrm{msec}$

26214

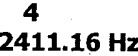

20.50 usec

$0.1520 \mathrm{sec}$

$2.0000 \mathrm{sec}$

16

$0.12 \mathrm{~Hz}$

0.00

0.00

90.00

100.00

single_pulse_dec

$1 \mathrm{H}$

$600.17 \mathrm{MH}$

$5.30 \mathrm{KHz}$

$5.47 \mathrm{~Hz}$

E:¥Personal Folder¥Cho¥Cho_phosphorylation_s

$0.00 \mathrm{KHz}$

$0.0 \mathrm{~Hz}$

0

o

$\mathrm{O} \mathrm{Hz}$ 


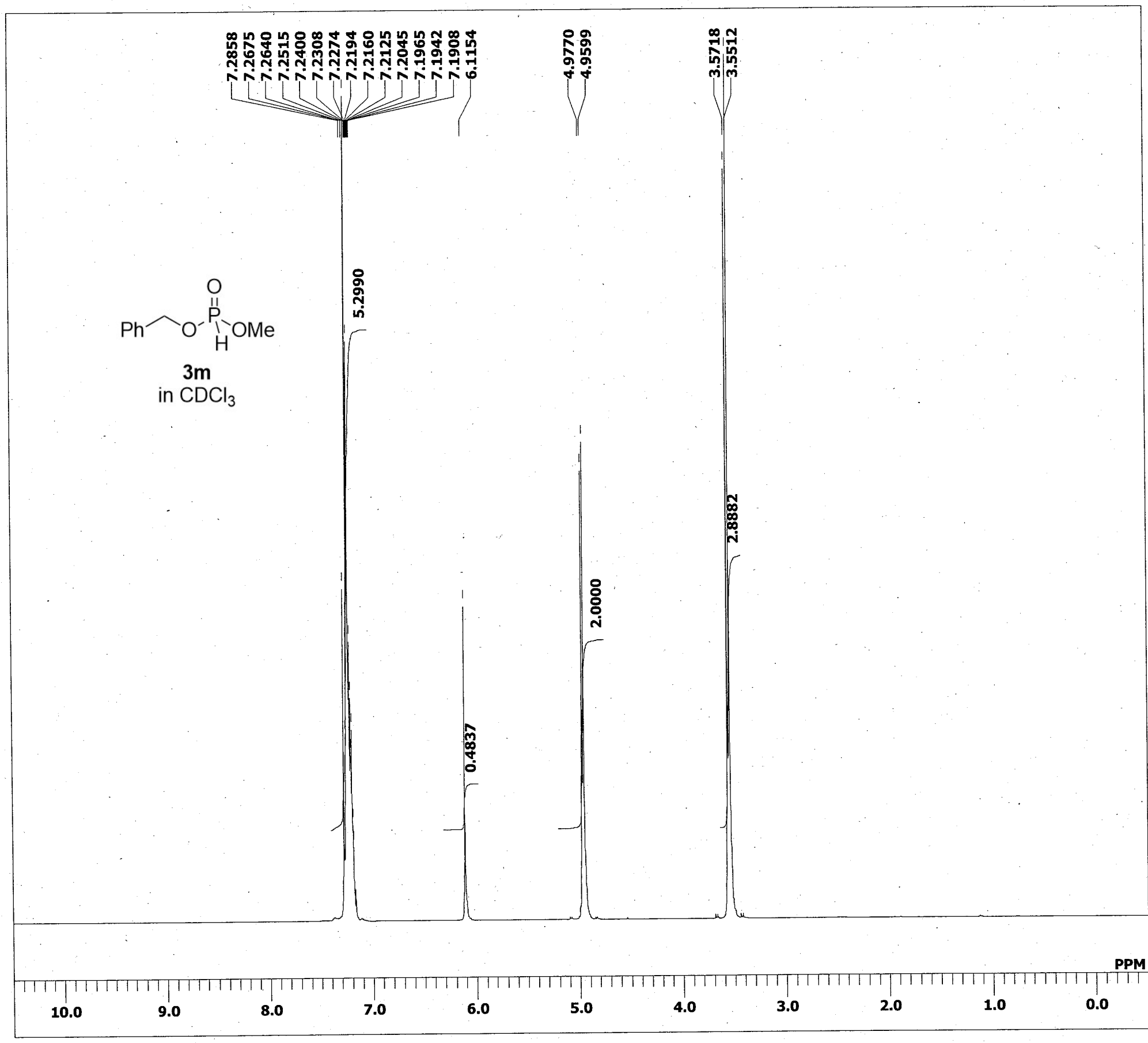

DATIM _ 27-12-2019 03:55:32

MENUF

OBNUC

OBNUC

OFR

OBFIN

PW1

$600.17 \mathrm{MHz}$

$5.30 \mathrm{KHz}$

$5.30 \mathrm{KHz}$
$5.47 \mathrm{~Hz}$

6.39 usec

0.00 usec

PREDL $\quad 0.00000 \mathrm{mse}$

IWT $\quad 1.0000 \mathrm{msec}$

POINT 13107

13107
SPO

TIMES $\quad 8$

FREOU $\quad 9008.87 \mathrm{~Hz}$

FLT

DELAY $\quad 10.88$ usec

RGAIN

T1

T2

T3

$\begin{array}{ll}100.00 \\ \text { EXMOD } & \text { single.pulse.ex }\end{array}$

EXPCM

IRNUC 1H

IFR

1H $600.17 \mathrm{MHz}$

$\quad 5.30 \mathrm{KHz}$

$\begin{array}{ll} & 5.47 \\ \text { IRRPW } & \mathbf{7 6} \\ \text { usec }\end{array}$

IRATN

DFILE E:¥Personal Folder*Cho\#Cho_phosphorylation_si

LKSET

$72.90 \mathrm{KHz}$

$53.8 \mathrm{~Hz}$

LKLEV

LAIN

CSPED

CSPED

FILDF

$1 \mathrm{H}$-NMR ( $\mathrm{CDCl} 3)$ ठ:

7.29-7.19 $(5 \mathrm{H}, \mathrm{m})$,

$6.12(0 \mathrm{H}, \mathrm{s})$

$4.97(2 \mathrm{H}, \mathrm{d}, \mathrm{J}=10.31 \mathrm{~Hz})$

$3.56(3 \mathrm{H}, \mathrm{d}, \mathrm{J}=12.37 \mathrm{~Hz})$

$3.56(3 \mathrm{H}, \mathrm{d}, \mathrm{J}=12.37 \mathrm{~Hz})$. 
E:*Personal Folder*Cho*Cho_phosphorylation_sub¥sub3k_carbon.als single pulse decoupled gated NOE

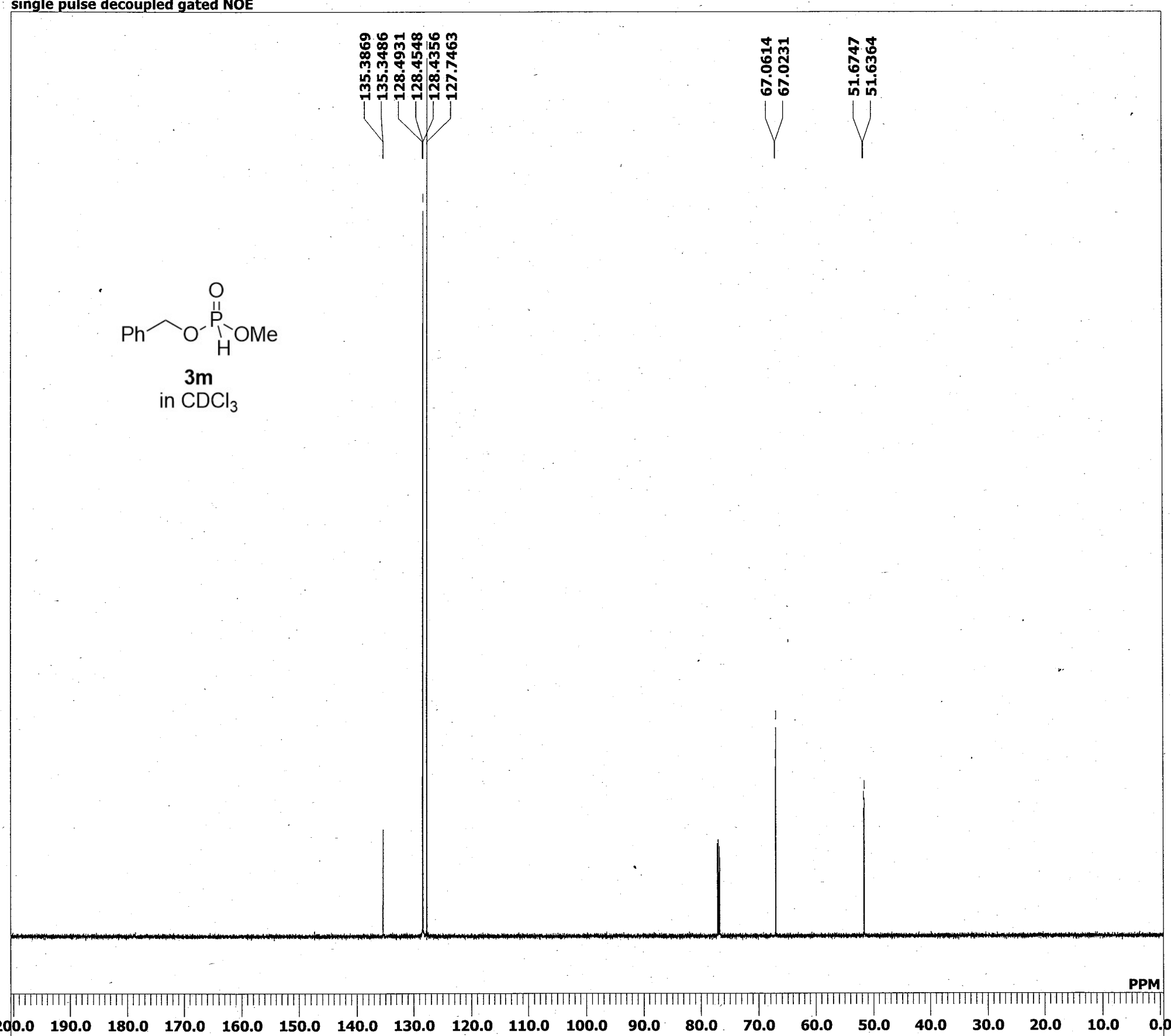

DATIM

MENUF

OBNUC

OFR

PW1

DEADT

PREDL

IWT

PPO

TPO

DUMY

DUMMY

REQU

DELAY

ACQTM

ADBIT

RGAIN

BF

T2

T3

EXMOD single_pulse_dec

IRN

IRSET

$1 \mathrm{H}$

IRFIN

$600.17 \mathrm{MHz}$

$5.30 \mathrm{KHz}$

$5.47 \mathrm{~Hz}$

IRATN 0

DFILE E:*Personal Folder*Cho*Cho_phosphorylation_si

\section{$72.90 \mathrm{KHz}$}

LKFIN $\quad 53.8 \mathrm{~Hz}$

LKLEV $\quad 0$

$\begin{array}{ll}\text { LLLEV } & 0 \\ \text { LGAIN } & 0\end{array}$

LKSIG

CSPED

FILDC

FILDF

13C -NMR (CDCl3) $\delta$ :

$135.37(\mathrm{OH}, \mathrm{d}, \mathrm{J}=\mathbf{5 . 7 8 \mathrm { Hz }}$,

$128.45(\mathrm{OH}, \mathrm{s})$,

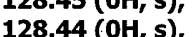

$127.75\left(\mathrm{OH}_{\mathrm{s}} \mathrm{s}\right)$

$67.04(0 \mathrm{OH}, \mathrm{d}, \mathrm{J}=5.78 \mathrm{~Hz})$

$51.66(0 \mathrm{H}, d, J=5.78 \mathrm{~Hz})$. 
E:*Personal Folder*Cho*Cho_phosphorylation_sub\#sub3k_phos.als

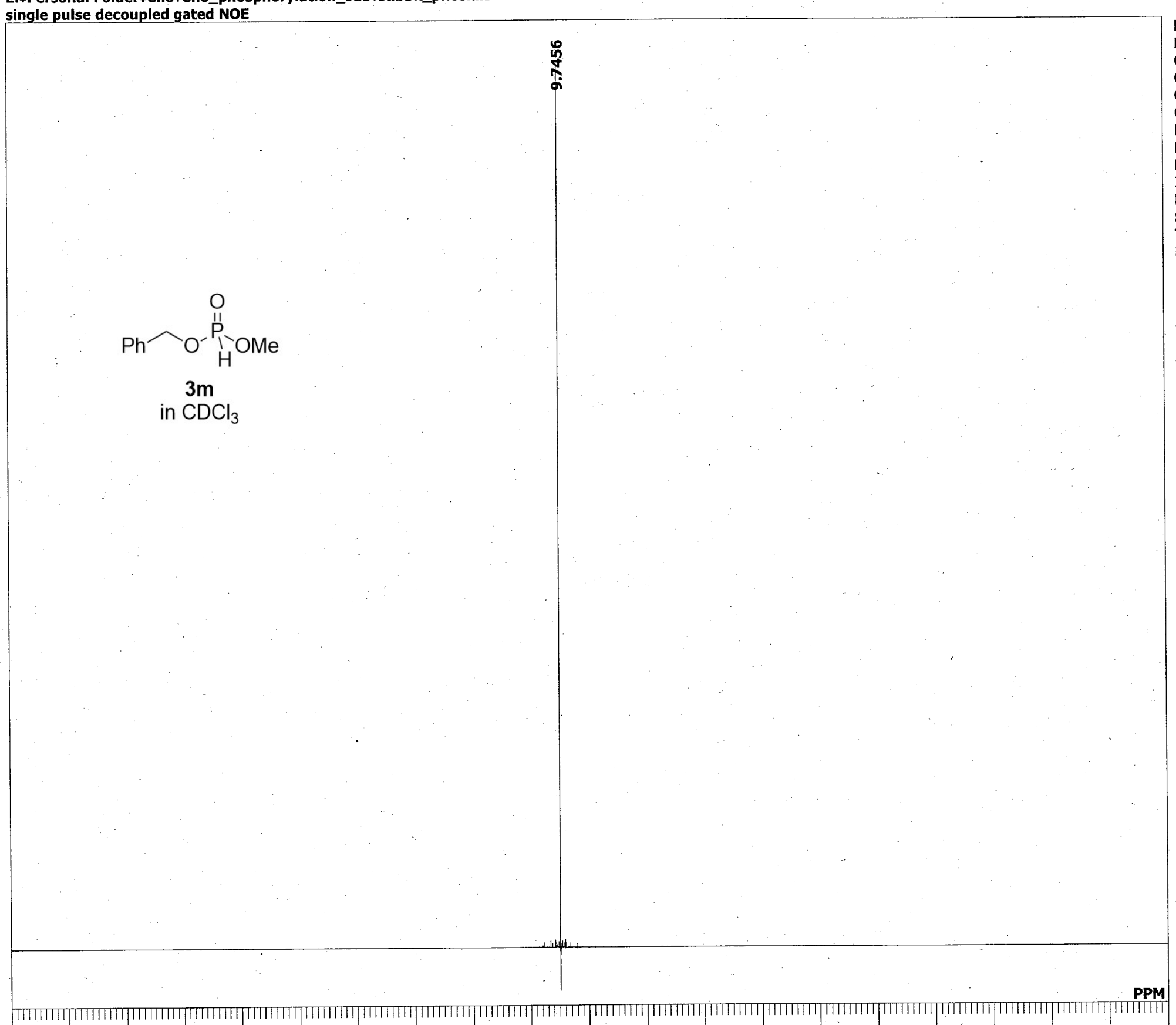

27-12-2019 04:00:05

OFR $242.95 \mathrm{MHz}$

$\quad 4.04 \mathrm{KHz}$

OBFIN $\quad 1.25 \mathrm{~Hz}$

PEADT $\quad 0.00$ usec

PREDL $\quad 0.00000 \mathrm{msec}$

WT $\quad 1.0000 \mathrm{msec}$

$\begin{array}{ll} & 1.0000 \\ \text { POINT } & \mathbf{2 6 2 1 4}\end{array}$

SPO $\quad 26214$

TIMES $\quad 49$

$172411.16 \mathrm{~Hz}$

FREQ

FLT

ACOTM

ACQTM
PD

ADBIT

BF

T1

T2

T4

EXMOD

EXPCM

IRNUC

IFR

IRFIN

IRRPW

IRATN

DFILE

SF.

LKSET

LKFIN

LKLEV

LAIN

CSPED

ILDC

FILDF
20.50 usec

$2.0000 \mathrm{sec}$

16
50

$0.12 \mathrm{~Hz}$

0.12
0.00
0.00

90.00

100.00

single_pulse_dec

$600.17 \mathrm{MHz}$

$5.30 \mathrm{KHz}$

usec

E:*Personal Folder¥Cho¥Cho_phosphorylation_s

$0.00 \mathrm{KHz}$

o

$\mathrm{O} \mathrm{Hz}$

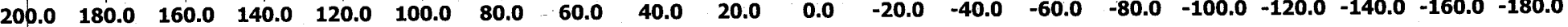


E:*Personal Folder*Lucałexp83-target-1 als

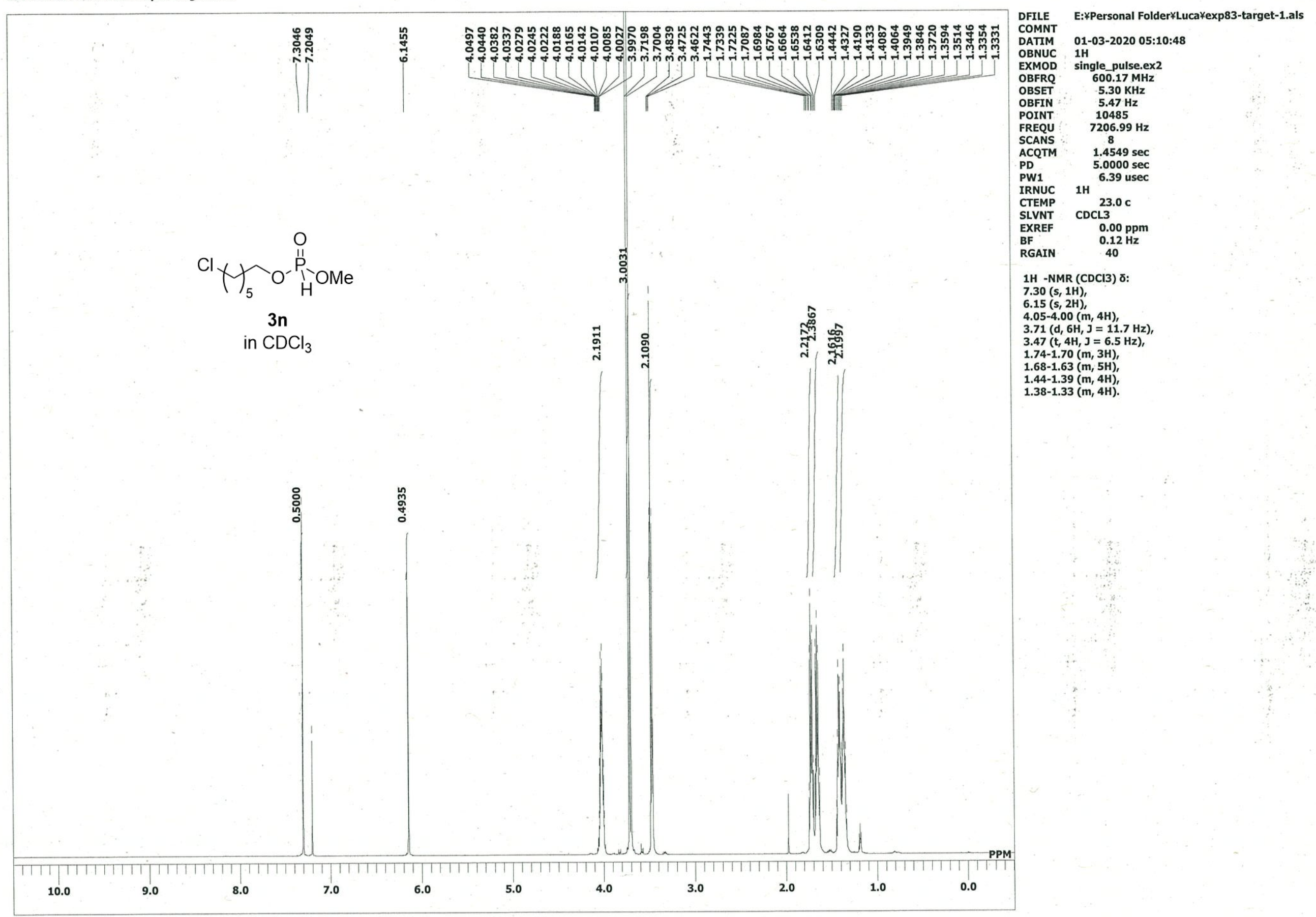




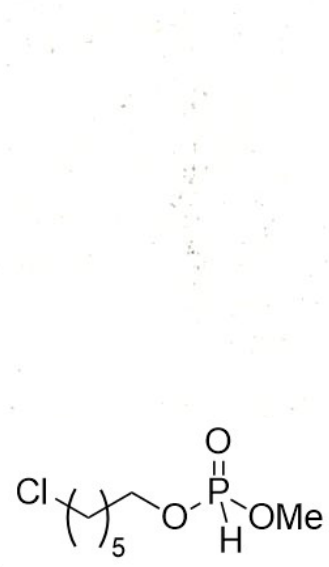

$3 n$

in $\mathrm{CDCl}_{3}$

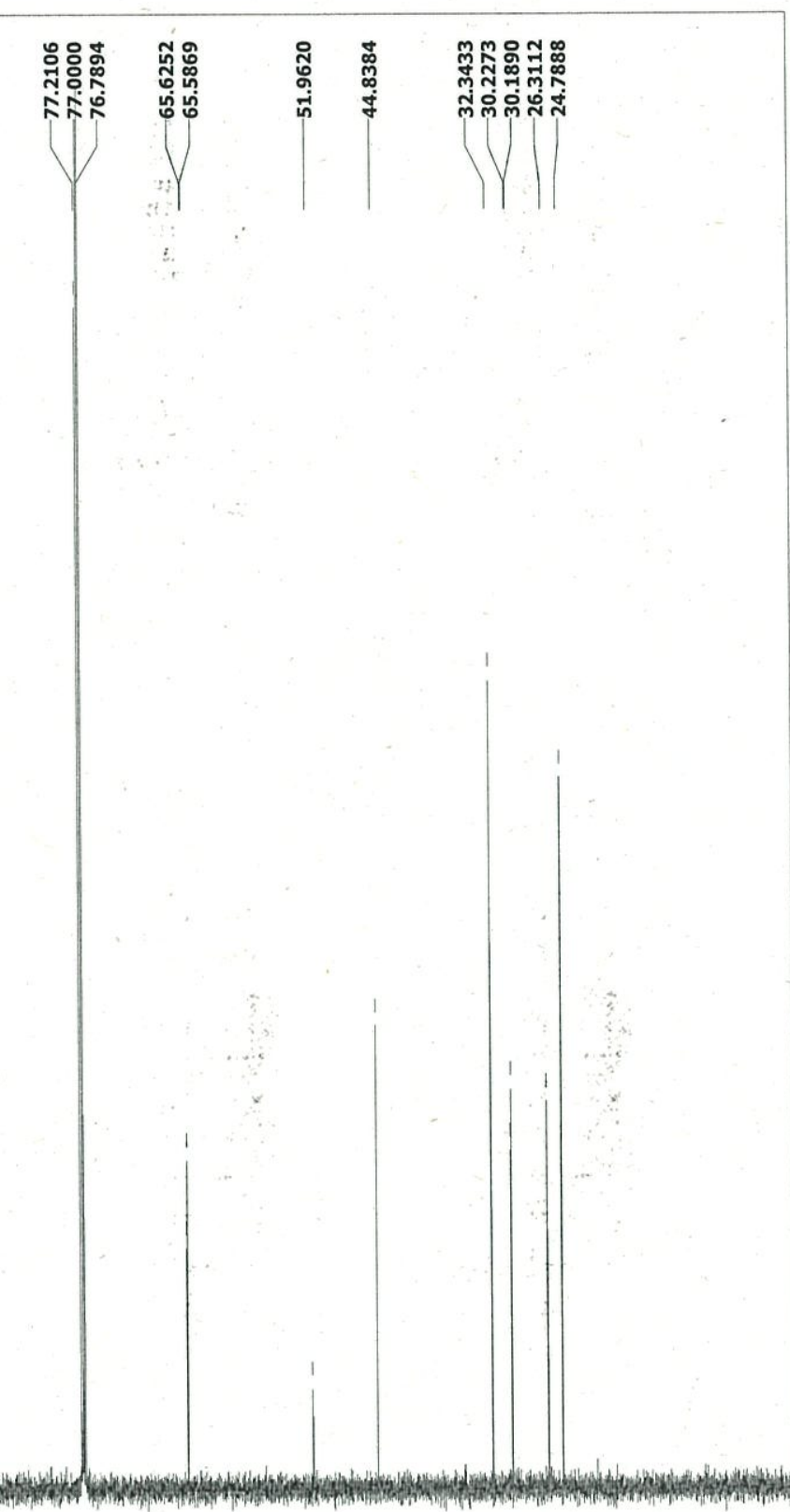

PPM
E:×Personal FolderyLucazexp83-target-13C-1.als

decoupled gated NOE

01-03-2020 05:33:25

DENUC $13 \mathrm{C}$

$\begin{array}{ll}\text { OBFRQ } & 150.92 \mathrm{MHz} \\ \text { OBSET } & 8.52 \mathrm{KHz}\end{array}$

OBFIN $\quad 8.52 \mathrm{KHz}$

POINT 26214

FREQU $\quad 37878.21 \mathrm{~Hz}$

305

$\begin{array}{ll}\text { PD } & 0.6921 \mathrm{sec} \\ \text { PD } & 2.0000 \mathrm{sec}\end{array}$

PW1 4.02 usec

IRNUC 1H

CTEMP $24.0 \mathrm{C}$

$\begin{array}{ll}\text { SLVNT } & \text { CDCL3 } \\ \text { EXREF } & 77.00 \mathrm{ppm}\end{array}$

$\begin{array}{ll}\text { EXREF } & 77.00 \mathrm{ppm} \\ \text { BF } & 0.12 \mathrm{~Hz}\end{array}$

$13 \mathrm{C}$-NMR (CDCI3) ठ:

$65.61(\mathrm{~d}, \mathrm{OH}, \mathrm{J}=5.8 \mathrm{~Hz})$

$44.84(\mathrm{~s}, \mathrm{OH})$

$32.34(s, 0 \mathrm{H})$,

$30.21(\mathrm{~d}, \mathrm{OH}, \mathrm{J}=5.8 \mathrm{~Hz})$,

$26.31(\mathrm{~s}, \mathrm{OH})$

$24.79(\mathrm{~s}, \mathrm{OH})$.

(2)


E:FPersonal Folder*Luca¥exp83-target-31P-1.als

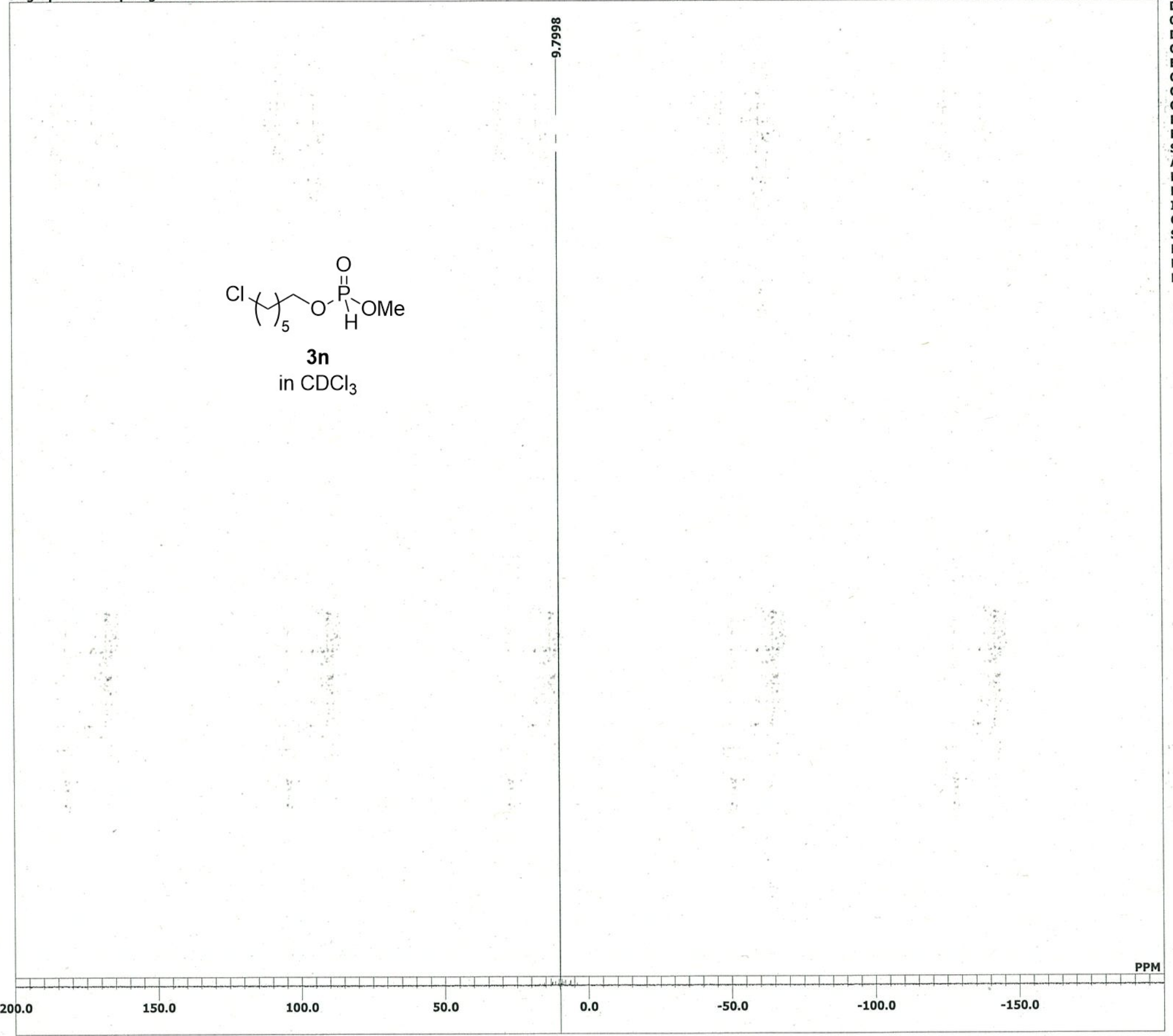

DAMT

DATIM 01-03-2020 05:17:54

single_pulse_dec

OBFRQ $242.95 \mathrm{MHz}$

26214

FREQU $\quad 172411.16 \mathrm{~Hz}$

157

$\quad 0.1520 \mathrm{sec}$

$\begin{array}{ll}2.0000 \mathrm{sec} \\ \text { PW1 } & 4.00 \mathrm{usec}\end{array}$

IRNUC 1H

CTEMP $23.6 \mathrm{C}$

SLVNT CDCL3

$\begin{array}{ll}\text { EXREF } & 0.00 \mathrm{ppm} \\ \text { BF } & 0.12 \mathrm{~Hz}\end{array}$

$31 \mathrm{P}$-NMR (CDCI3) ठ: $9.80(s, \mathrm{OH})$. 
11-03-2020 23:44:20

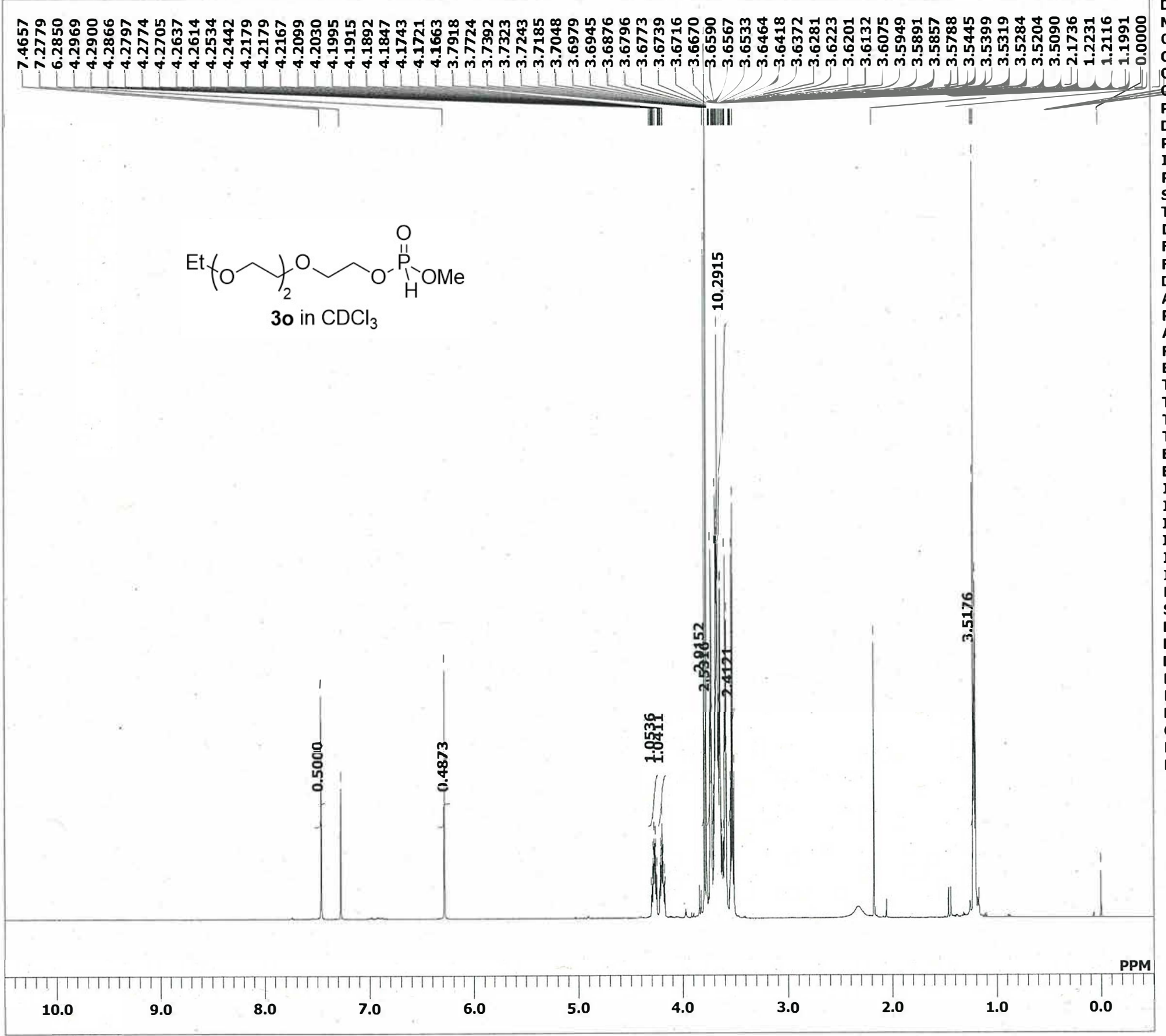

$600.17 \mathrm{MHz}$

$5.30 \mathrm{KHz}$

$5.47 \mathrm{~Hz}$

6.39 usec

$0.00000 \mathrm{msec}$

$0.00000 \mathrm{msec}$

$1.0000 \mathrm{~ms}$

SPO $\quad 13107$

TIMES 8

1

FLT

DELAY 10.88 use

$1.4549 \mathrm{sec}$

ADBIT $\quad 16$

16
RGAIN $\quad 38$

BF $0.12 \mathrm{~Hz}$

$\mathrm{T} 1 \quad 0.00$

$\begin{array}{ll}0.00 \\ 13 & 0000\end{array}$

T4 $\quad 100.00$

EXMOD single_pulse.ex2

EXPCM

IRNUC

IRNUC

IFR

$1 \mathrm{H}$

IRFIN $\quad 5.47 \mathrm{~Hz}$

76 usec

DFILE E:¥Personal Folder¥Saito-sub¥Saito¥Phosphonylz

SF

LKSET $\quad 72.90 \mathrm{KHz}$

KFIN $\quad 53.8 \mathrm{~Hz}$

LKLEV 0

0

LKPHS

FILDC

FILDF

$1 \mathrm{H}$-NMR ( $\mathrm{CDCl} 3) \delta$

$6.88(\mathrm{OH}, \mathrm{d}, \mathrm{J}=709.33 \mathrm{~Hz})$

4.30-4.24 (1H, $\mathrm{m})$,

$3.78(3 \mathrm{H}, \mathrm{d}, \mathrm{J}=11.68 \mathrm{~Hz})$

$3.73(3 \mathrm{H}, \mathrm{q}, \mathrm{J}=4.12 \mathrm{~Hz})$,

3.70-3.58 (10H, m),

$1.21(4 \mathrm{H}, \mathrm{t}, \mathrm{J}=7.22 \mathrm{~Hz})$.
. 
E:¥Personal Folder¥Saito-sub¥Saito¥Phosphonylation¥polyether-31C.als

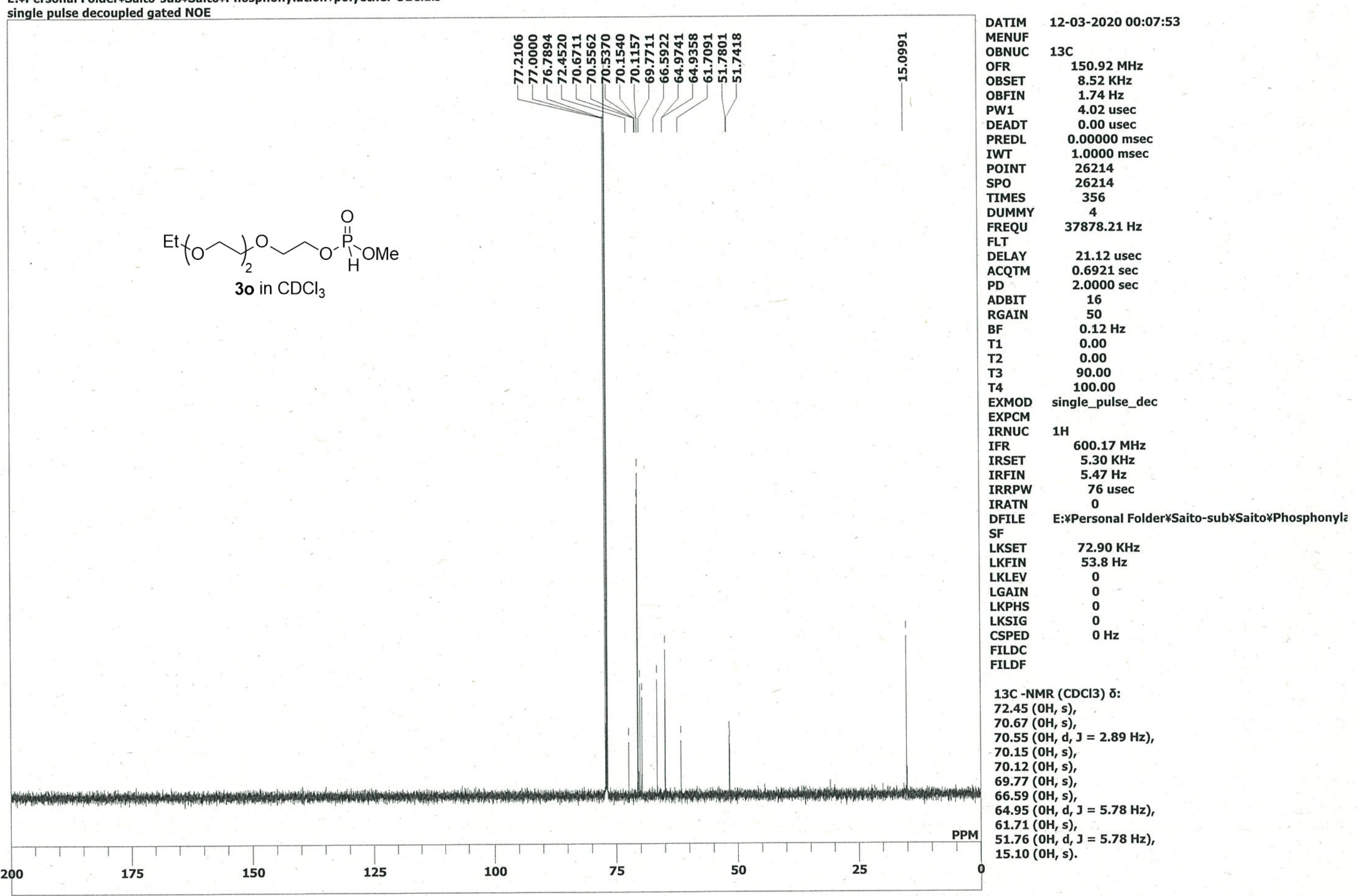


E:¥Personal Folder¥Saito-sub¥Saito*Phosphonylation¥polyether-31P.als

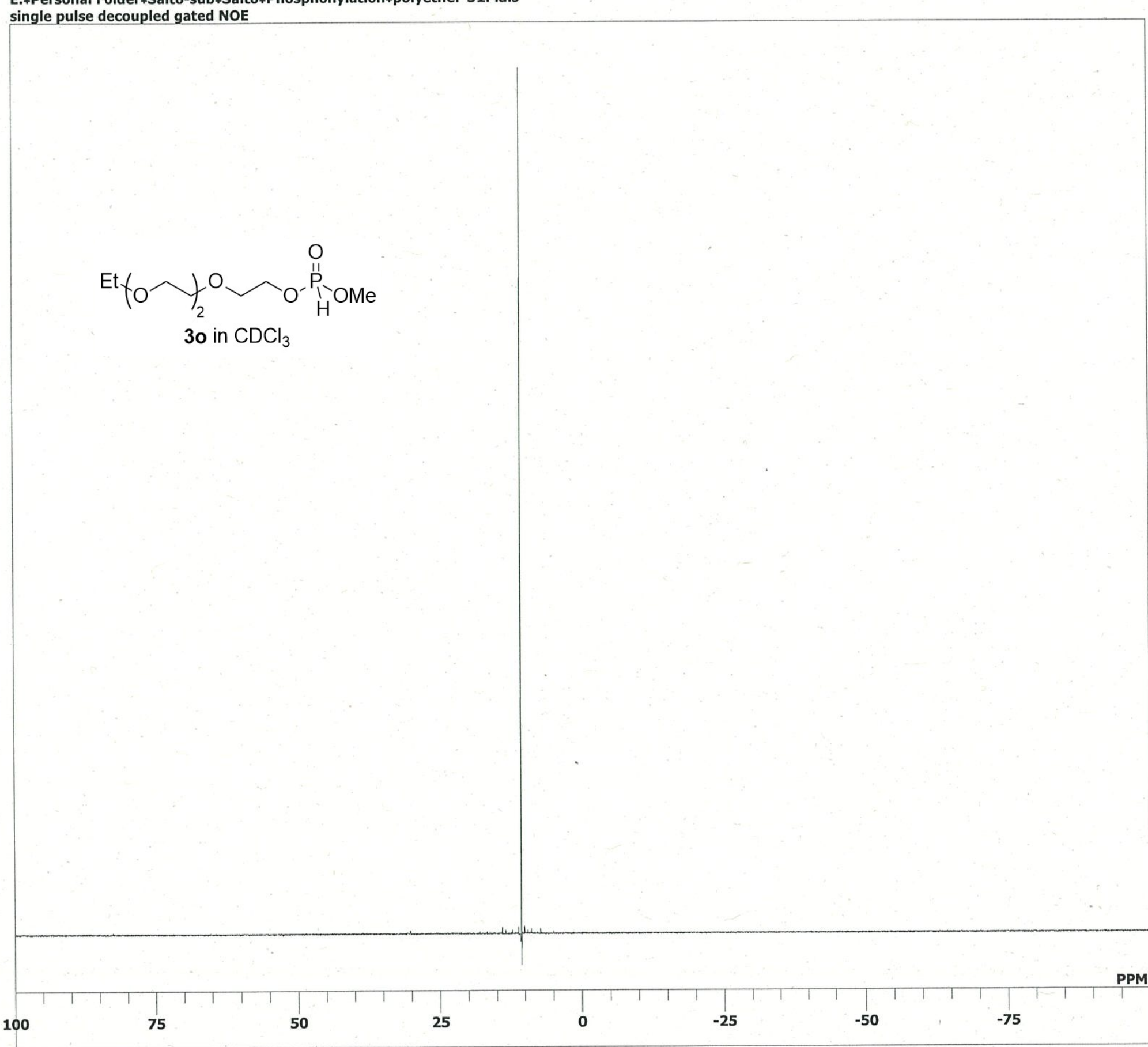

11-03-2020 23:49:54

BNUC 31P

$\quad 242.95 \mathrm{MHz}$

OBSET $\quad 4.04 \mathrm{KHz}$

$\begin{array}{ll}\text { OBFIN } \quad & 1.25 \mathrm{~Hz}\end{array}$

PW1 4.00 usec

DEADT $\quad 0.00$ usec

PREDL $\quad 0.00000 \mathrm{msec}$

IWT $\quad 1.0000 \mathrm{msec}$

POINT 26214

26214

TI

4
FREQU $\quad 172411.16 \mathrm{~Hz}$

$172411.16 \mathrm{~Hz}$

DELAY 20.50 usec

ACQTM $2.1520 \mathrm{sec}$

ADBIT $\quad 16$

RGAIN $\quad 50$

BF $\quad 0.12 \mathrm{~Hz}$

$\begin{array}{ll}\mathrm{BF} & 0.00 \\ \mathrm{~T} 1 & 0.00\end{array}$

T2 0.00

T4 $\quad 90.00$

EXMOD single_pulse_dec

EXPCM

IRNUC 1H

IFR $\quad 600.17 \mathrm{MHz}$

IRSET $\quad 5.30 \mathrm{KHz}$

IRFIN $\quad 5.47 \mathrm{~Hz}$

IRRPW 76 USec

IRATN

DFILE E:*Personal Folder¥Saito-sub¥Saito¥Phosphonyla

LKSET $\quad 0.00 \mathrm{KHz}$

LKFIN

LKLEV

LGAIN 0

LKPHS 0

CSPED $\quad 0 \mathrm{~Hz}$

FILDF 


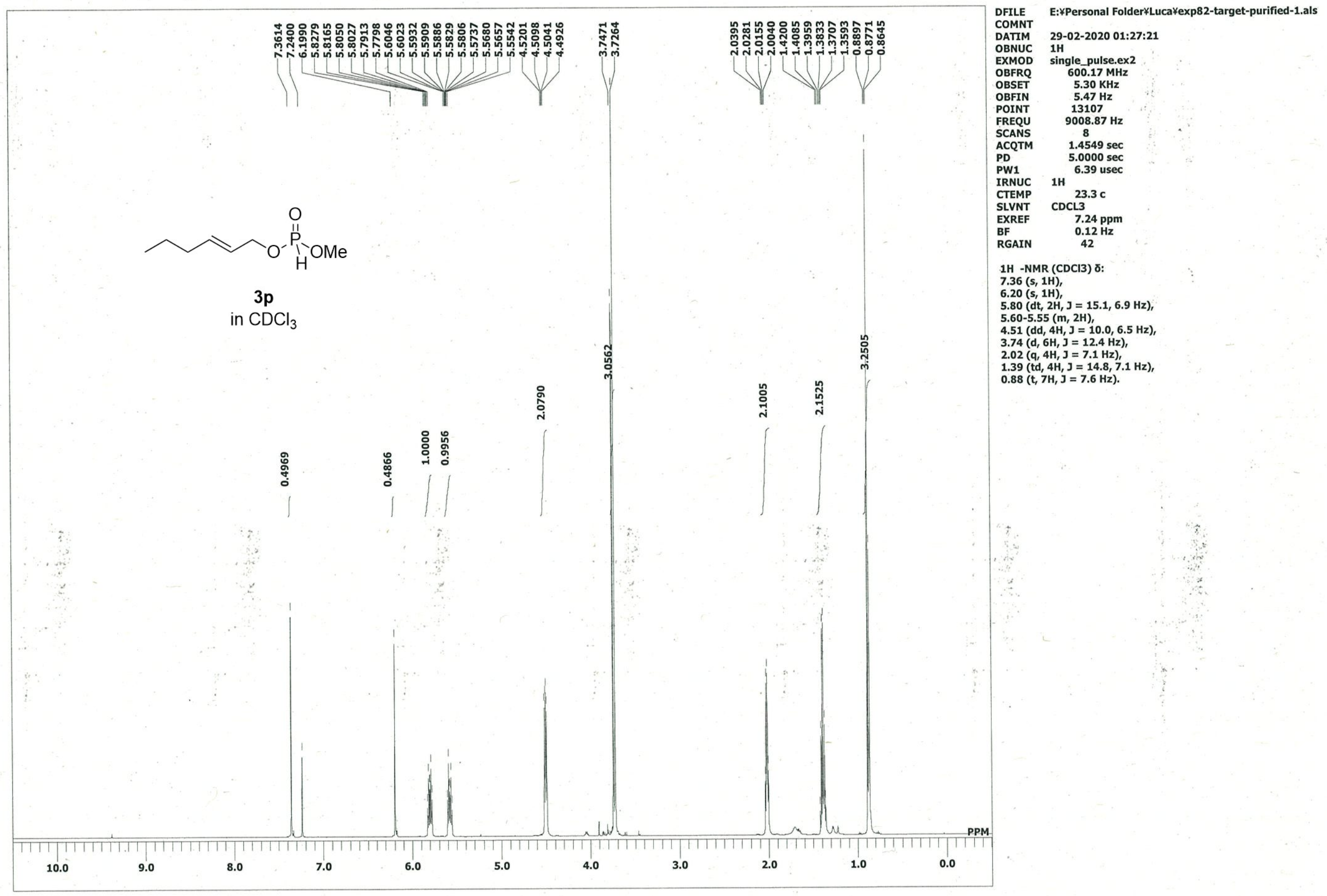


E:XPersonal Folder*Lucałexp82-target-purified-13C-1.als single pulse decoupled gated $\mathrm{NOE}$
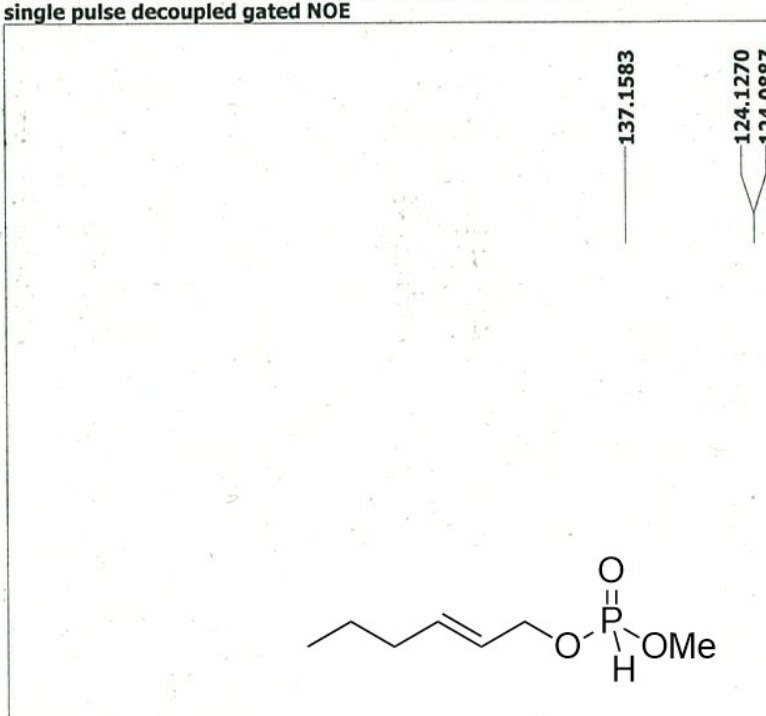

部

$3 p$

in $\mathrm{CDCl}_{3}$

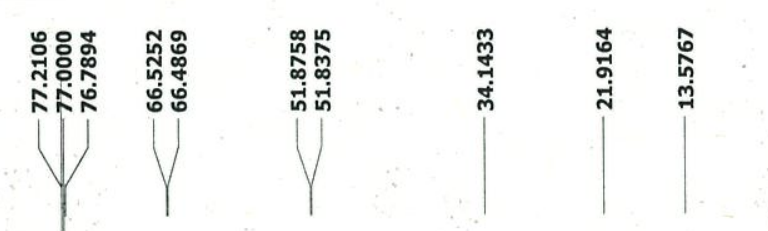

COMNT single pulse decoupled gated NOE

DATIM 29-02-2020 01:50:10

OBNUC $13 \mathrm{C}$

EXMOD single_pulse_de

OBFRQ $\quad 150.92 \mathrm{MHz}$

OBSIN $\quad 8.52 \mathrm{KHZ}$

POINT 26214

FREQU $\quad 37878.21 \mathrm{~Hz}$

SCANS $\quad 302$

$\quad 0.6921 \mathrm{sec}$

$\begin{array}{lr}\text { PD } & 2.0000 \mathrm{sec} \\ \text { PW1 } & 4.02 \mathrm{usec}\end{array}$

IRNUC 1H

CTEMP $24.1 \mathrm{c}$

$\begin{array}{ll}\text { SLVNT } & \text { CDCL3 } \\ \text { EXREF } & 77.00 \mathrm{ppm}\end{array}$

$\begin{array}{lc}\text { EXREF } & 77.00 \mathrm{ppm} \\ \text { BF } & 0.12 \mathrm{~Hz}\end{array}$

C13) $\delta:$

13C -NMR (CDCl3)
$137.16(\mathrm{~s}, \mathrm{OH})$,

$124.11(\mathrm{~d}, \mathrm{OH}, \mathrm{J}=5.8 \mathrm{~Hz})$,

$66.51(\mathrm{~d}, \mathrm{OH}, \mathrm{J}=5.8 \mathrm{~Hz})$,
$51.86(\mathrm{~d}, \mathrm{OH}, \mathrm{J}=5.8 \mathrm{~Hz})$

$34.14(\mathrm{~s}, \mathrm{OH})$,

$21.92(s, \mathrm{OH})$,
$13.58(\mathrm{~s}, \mathrm{OH})$.

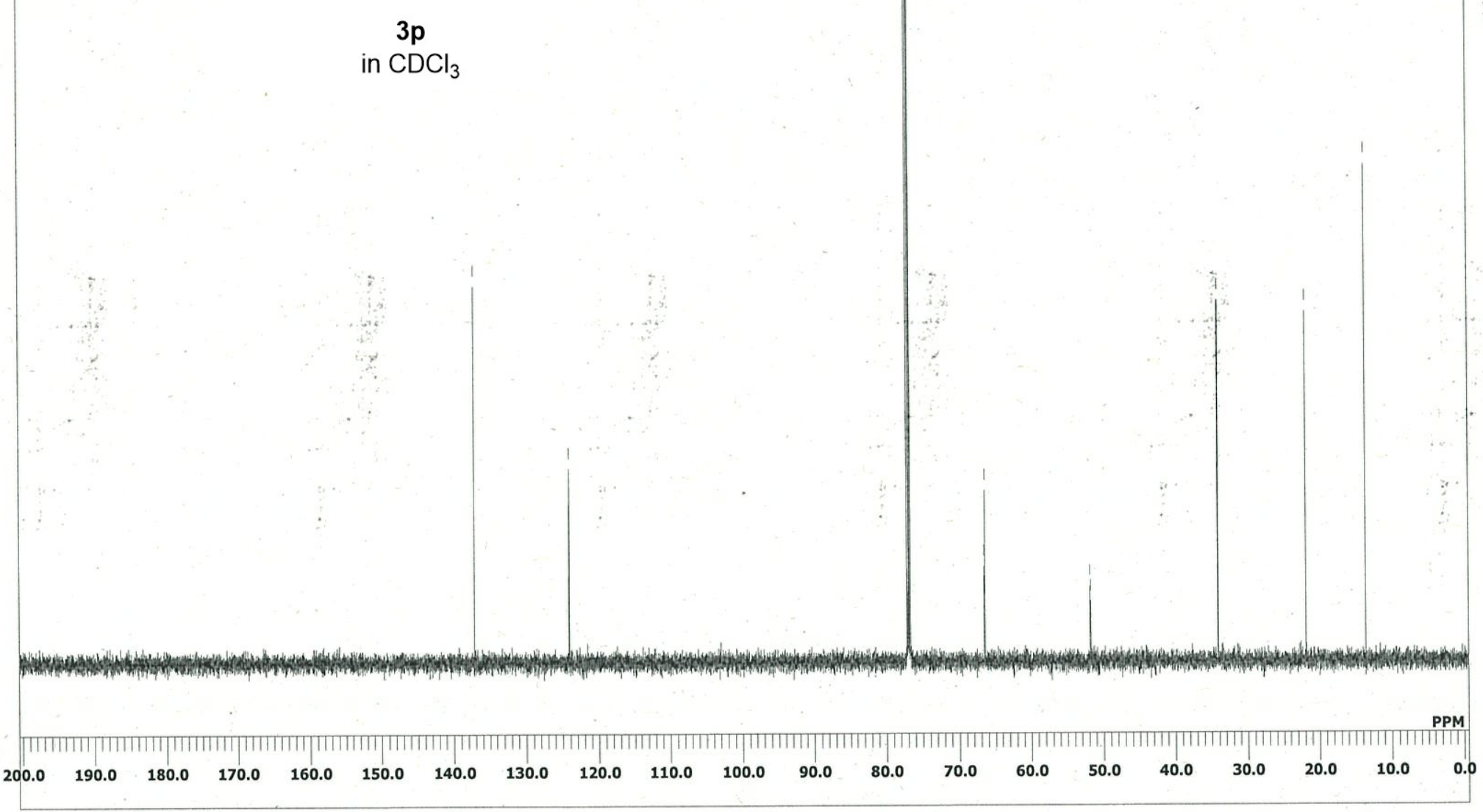


E:*Personal Folder*Lucałexp82-target-purified-31P.als

single pulse decoupled gated NOE

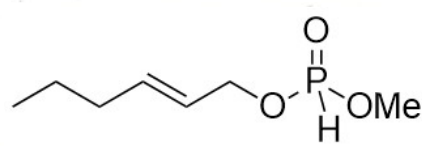

$3 p$

in $\mathrm{CDCl}_{3}$
E:×Personal FolderyLucayexp82-target-purified-31P als

DATMM 29-02-2020 01:34:41

OBNUC $31 \mathrm{P}$

$\begin{array}{ll}\text { EXMFR } & \text { single_pulse_dec } \\ \text { OB2.95 MHz }\end{array}$

OBSET $\quad 4.04 \mathrm{KHz}$

26214

FREQU $172411.16 \mathrm{~Hz}$

SCANS 153

$\quad 0.1520 \mathrm{sec}$

$\begin{array}{ll}2.0000 \mathrm{sec} \\ \text { PW1 } & 4.00 \mathrm{usec}\end{array}$

IRNUC 1H

CTEMP $23.8 \mathrm{C}$

SLVNT CDCL3

$\begin{array}{ll}\text { EXREF } & 0.00 \mathrm{ppm} \\ \text { BF } & 0.12 \mathrm{~Hz}\end{array}$

$31 \mathrm{P}$-NMR (CDCI3) ठ: $9.72(\mathrm{~s}, \mathrm{OH})$. 


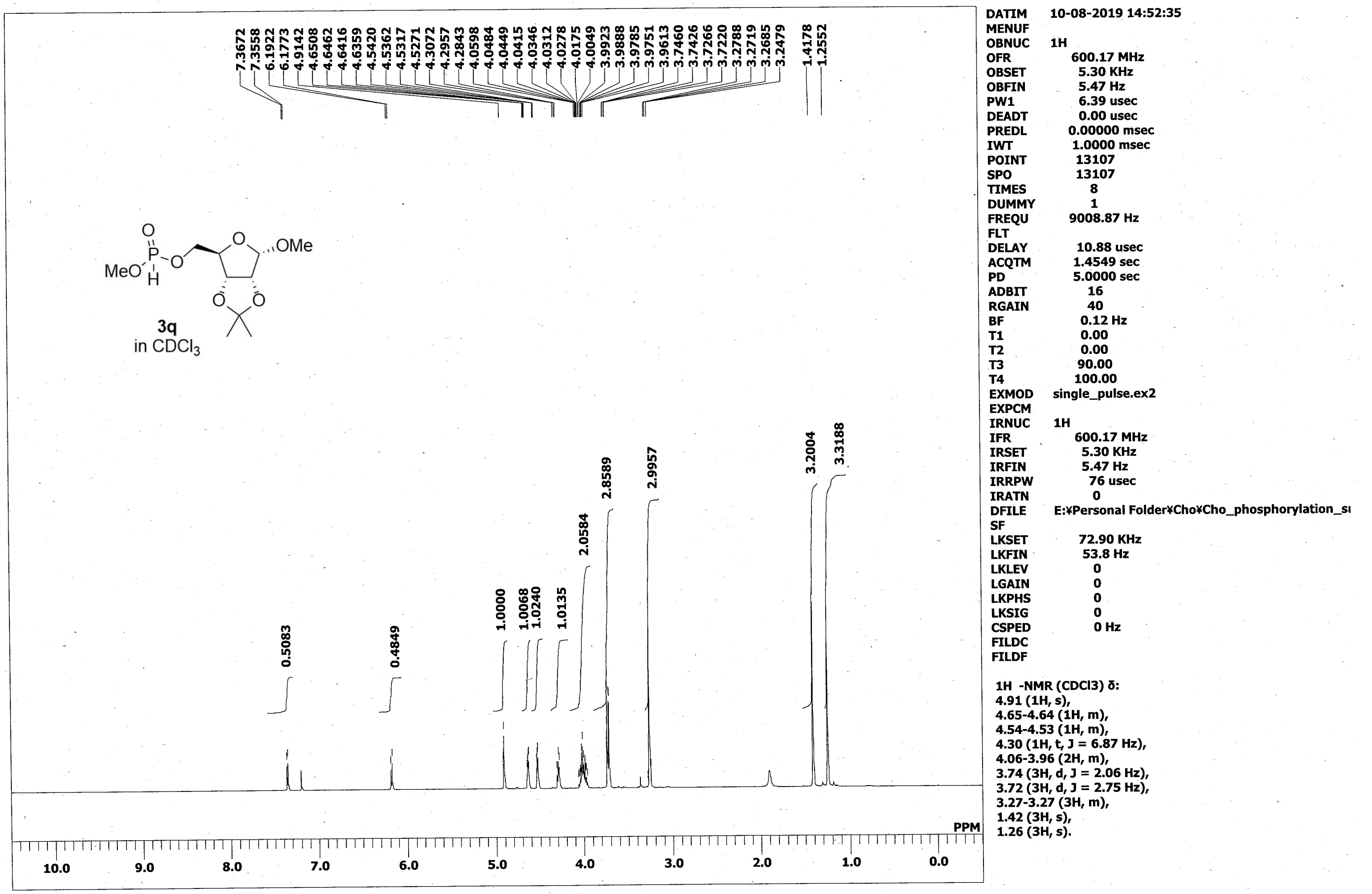


E:*Personal Folder*Cho*Cho_phosphorylation_sub*sub3o_carbon.als

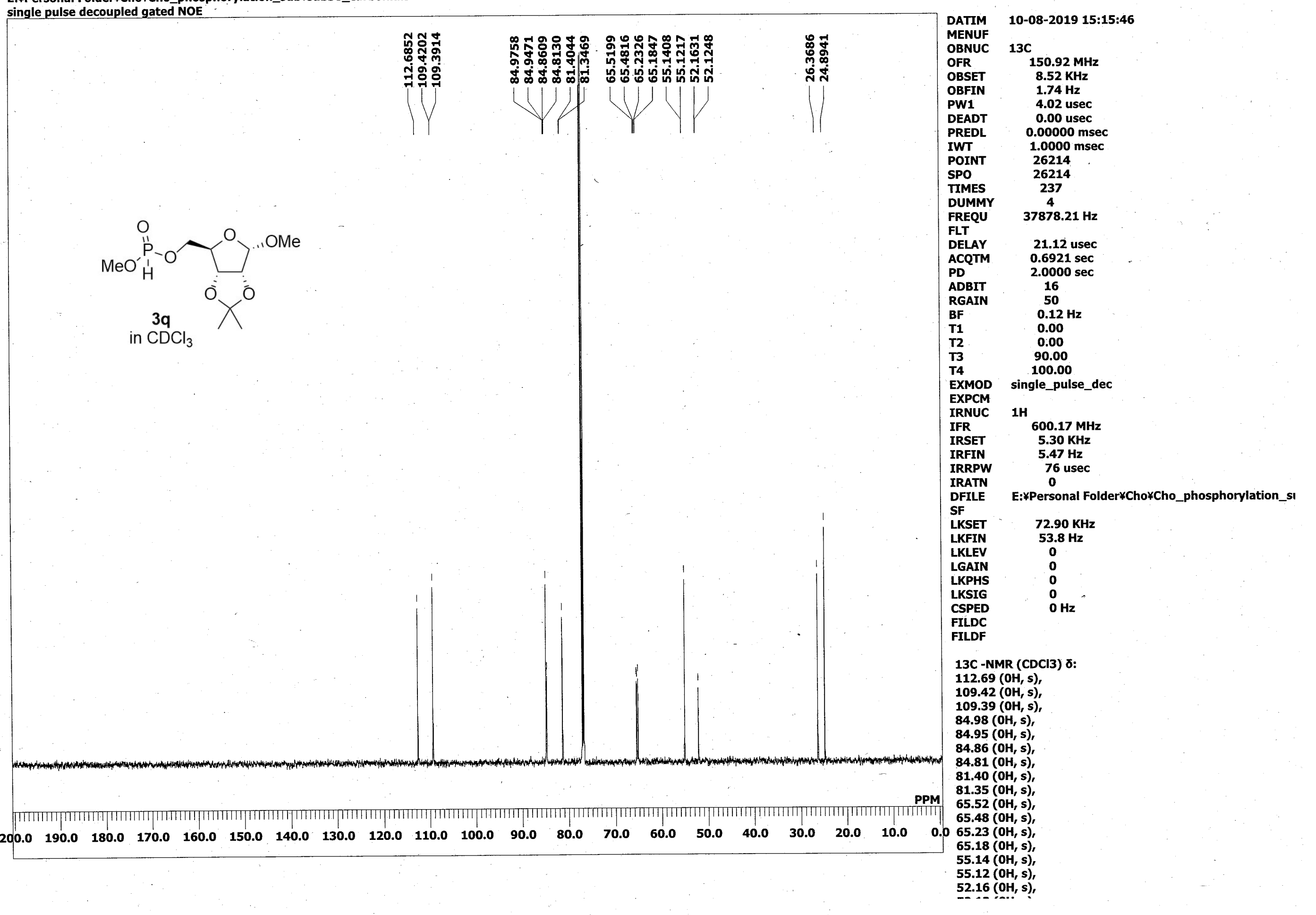


E:*Personal Folder*Cho¥Cho_phosphorylation_sub¥sub30_phos.als

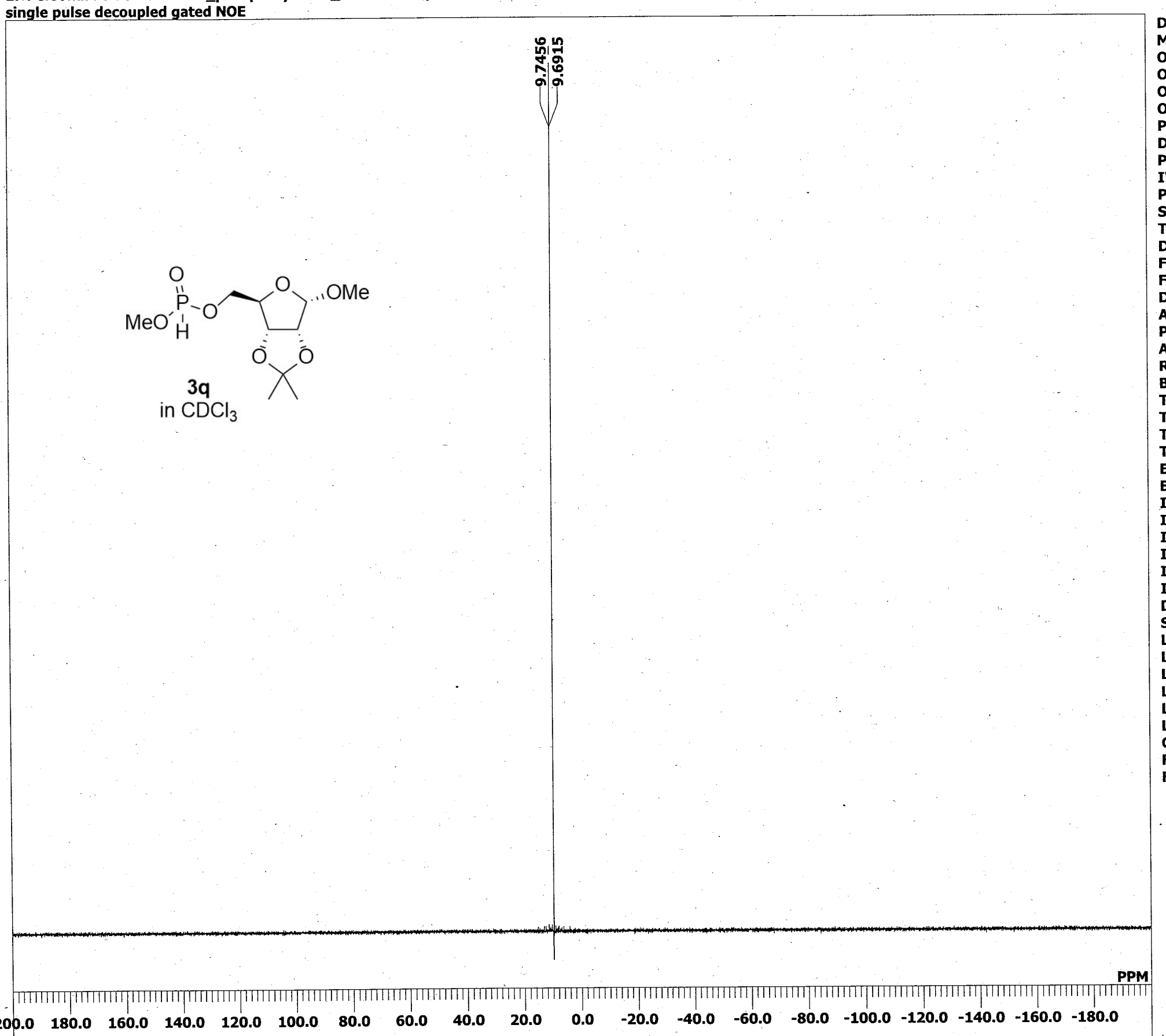

10-08-2019 15:19:50

\section{MENUF}

OBNUC 31P

OFR $\quad 242.95 \mathrm{MHz}$

OBSET $\quad 4.04 \mathrm{KHz}$

PW1 $\quad 4.25 \mathrm{~Hz}$

DEADT $\quad 4.00$ usec

PREDL $\quad 0.00000 \mathrm{msec}$

IWT $\quad 1.0000 \mathrm{msec}$

POINT $\quad 26214$
PRT

$\begin{array}{ll}\text { SPO } & \mathbf{2 6 2 1 4} \\ & \mathbf{2 6 2 1 4}\end{array}$

TIMES 61

DUMMY 4 4

FLT

ACQTM
PD
ADBIT

RGAIN

1

T1
T2
T3

T3

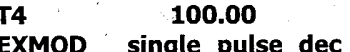

XPCM

EXNUC

IFR

1H

20.50 usec

$0.1520 \mathrm{sec}$

$1600 \mathrm{sec}$

16

$0.12 \mathrm{~Hz}$

0.00

$\quad 600.17 \mathrm{MHz}$

$\begin{array}{ll} & 5.30 \mathrm{KHz} \\ \text { IRFIN } & 5.47 \mathrm{~Hz}\end{array}$

$\quad 76$ usec

IRATN 0

E:*Personal Folder*Cho*Cho_phosphorylation_s

$0.00 \mathrm{KHz}$

$0.0 \mathrm{~Hz}$

$0.0 \mathrm{~Hz}$

CSPED

FILDF

$31 \mathrm{P}$-NMR ( $\mathrm{CDCl} 3)$ ס:

$9.75(\mathrm{OH}, \mathrm{s})$,
$9.69(\mathrm{OH}, \mathrm{s})$.

0

o

$\mathrm{OHz}$ 


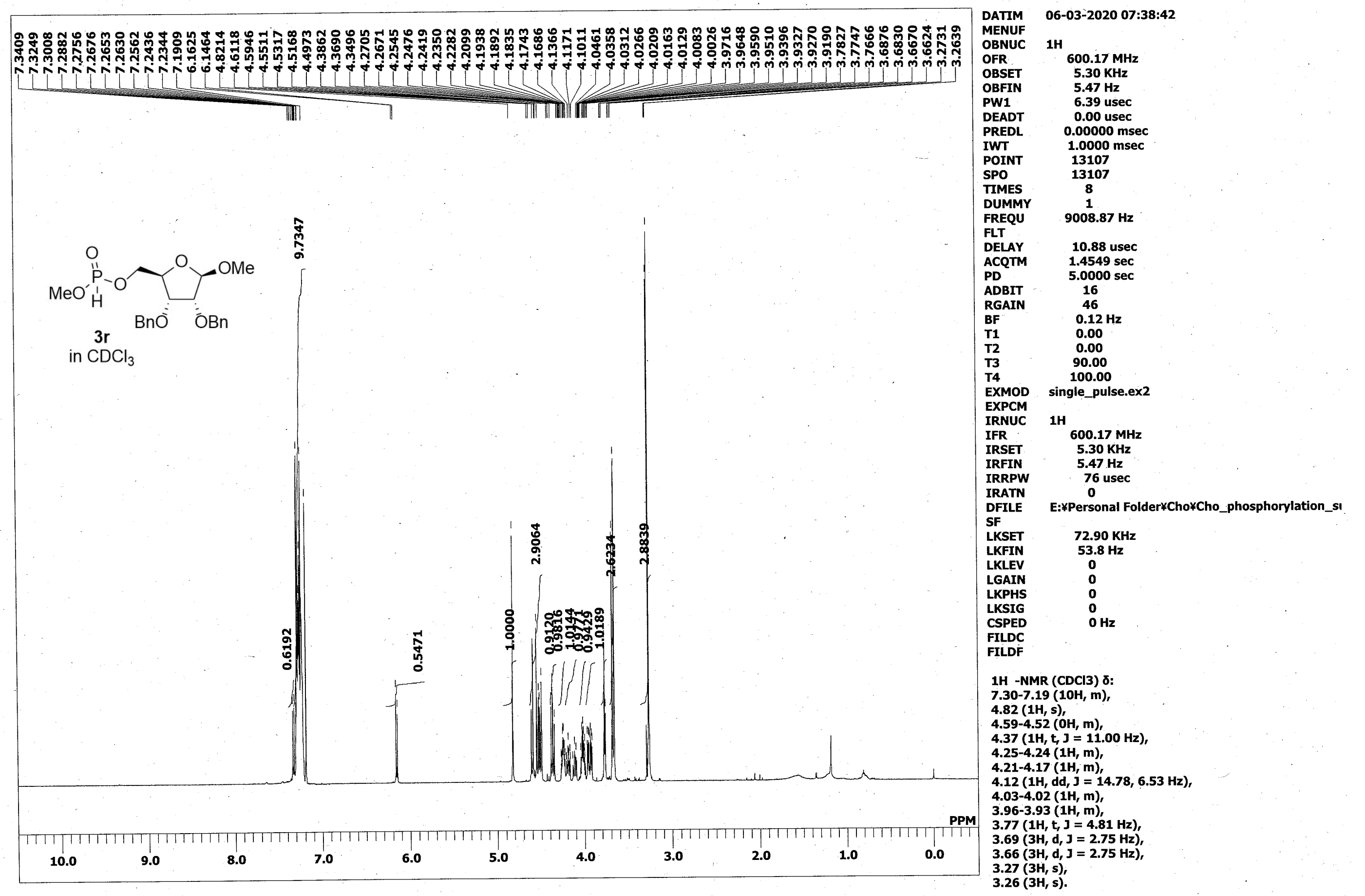




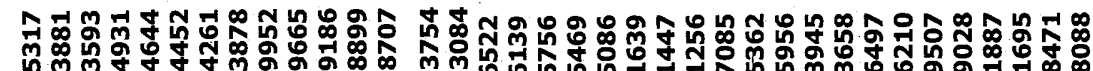 \\ mं
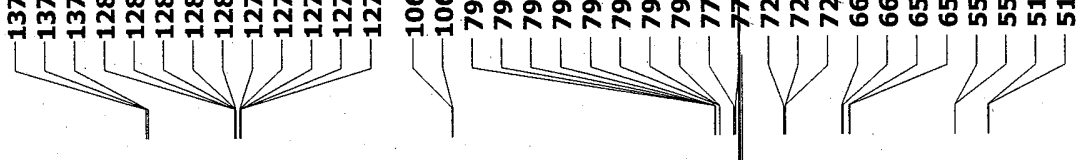

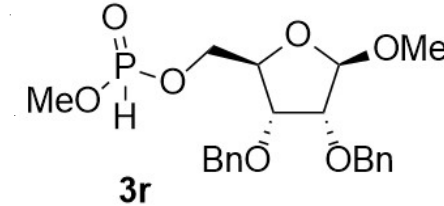

in $\mathrm{CDCl}_{3}$

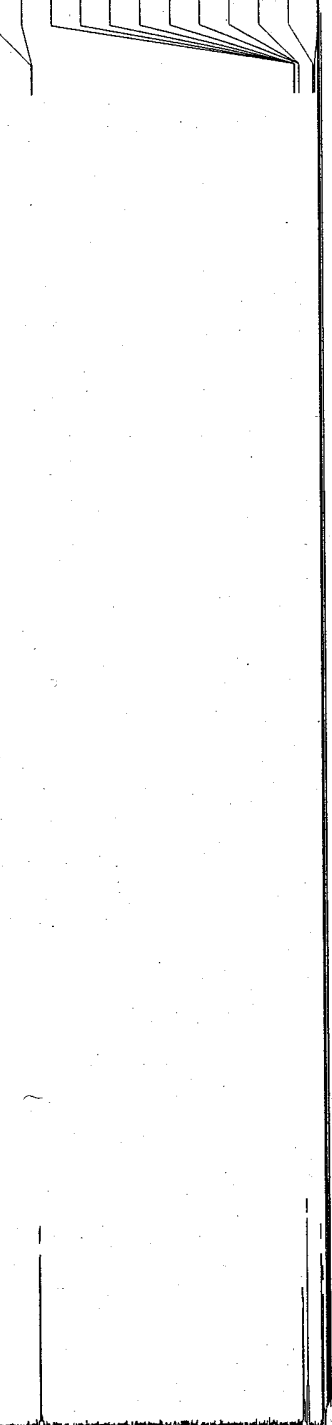

DATIM

MENUF

OBNUC

OBSET

OBFIN

PW1

DEADT

PREDL

IWT

POINT

SPO

TIMES

DUMMY

FREQU

FLT

DELAY

ACQTM

ADBIT

RGAIN

BF

B1

T2

T3

EXMOD

EXPCM

IRNUC

IFR
IRSET
IRFIN

IRFIN

IRRPW

IRATN

DFILE

SF

LKFIN

LLEV

LGAIN

LKPHS

LSIG

CSPED

FILDC

FILDF

13C -NMR ( $\mathrm{CDCl} 3) \delta$ :

$137.53(\mathrm{OH}, \mathrm{s})$,

$137.39(\mathrm{OH}, \mathrm{s})$,

$137.36(\mathrm{OH}, \mathrm{s})$,

128.49-128.39 (OH, m)

128.00-127.87 (OH, m),

$106.38(0 \mathrm{H}, \mathrm{s})$,

$106.31(\mathrm{OH}, \mathrm{s})$,

$79.65(\mathrm{OH}, \mathrm{s})$,

$79.61(\mathrm{OH}, \mathrm{s})$,

PPM $79.55(\mathrm{OH}, \mathrm{s})$

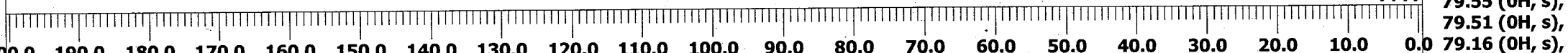

$79.13(\mathrm{OH}, \mathrm{s})$,

$77.71(\mathrm{OH}, \mathrm{s})$,

$77.54(\mathrm{OH}, \mathrm{s})$,

$72.60(\mathrm{OH}, \mathrm{s})$,
$150.92 \mathrm{MHz}$

$1.52 \mathrm{KHz}$

4.02 usec

$0000 \mathrm{msec}$

$0000 \mathrm{msec}$

6214

$7878.21 \mathrm{~Hz}$

usec

50

$0.12 \mathrm{~Hz}$

0.00

90.00

100.00

e_pulse_dec

600.17 MHz

$5.30 \mathrm{KHz}$

.47

76 usec

Folder*Cho*Cho_phosphorylation_si

$\mathrm{KHz}$

.8 $\mathrm{Hz}$

0

$\mathrm{Hz}$ 
E:*Personal Folder*Cho*Cho_phosphorylation_sub¥̇sub3p_phos.als single pulse decoupled gated NOE

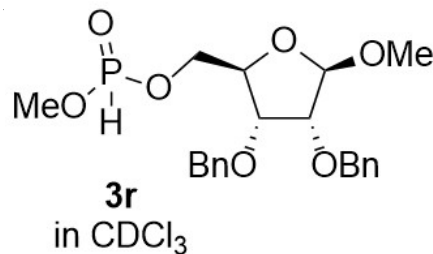

DATIM

OBNUC

OFR

OBSET

OBFIN

PW1

PREDL

IWT

POINT

SPO

DUMMY

FREQU

FLT

DELAY

ACQTM

ADBIT

RGAIN

BF

T1

T2

$\mathrm{T} 3$
$\mathrm{~T} 4$

EXMOD

EXPCM

IRNUC

IFR

IRFIN

IRRPW

IRATN

DFILE

SF

LKSET

LKFIN

LKLEV

LGAIN

LKPHS

LKSIG

CSPED

FILDC

FILDF
242.95 MHz

$4.04 \mathrm{KHz}$
$1.25 \mathrm{~Hz}$

$4.00 \mathrm{usec}$
$0.00 \mathrm{usec}$

0.00 usec

$0.00000 \mathrm{msec}$

$1.0000 \mathrm{msec}$

26214

$172411.16 \mathrm{~Hz}$

20.50 usec

$0.1520 \mathrm{sec}$

$.0000 \mathrm{sec}$

16
50

$0.12 \mathrm{~Hz}$

0.00

0.00

90.00

100.00

single_pulse_dec

1H

$600.17 \mathrm{MHz}$

$5.30 \mathrm{KHz}$

$5.47 \mathrm{~Hz}$

76 usec

*Personal Folder*Cho*Cho_phosphorylation_st

$0.00 \mathrm{KHz}$

$0.0 \mathrm{~Hz}$

0

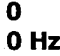

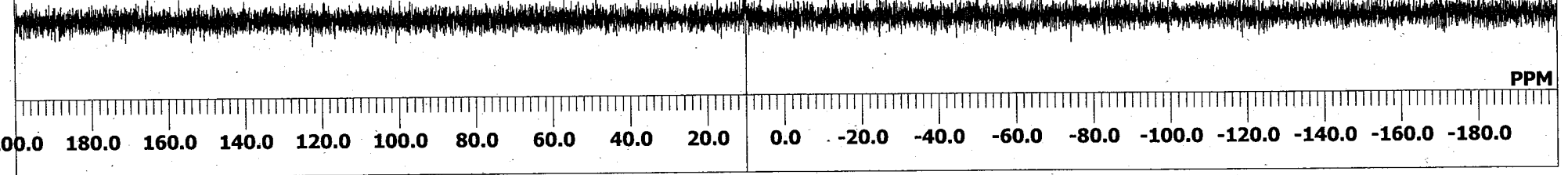




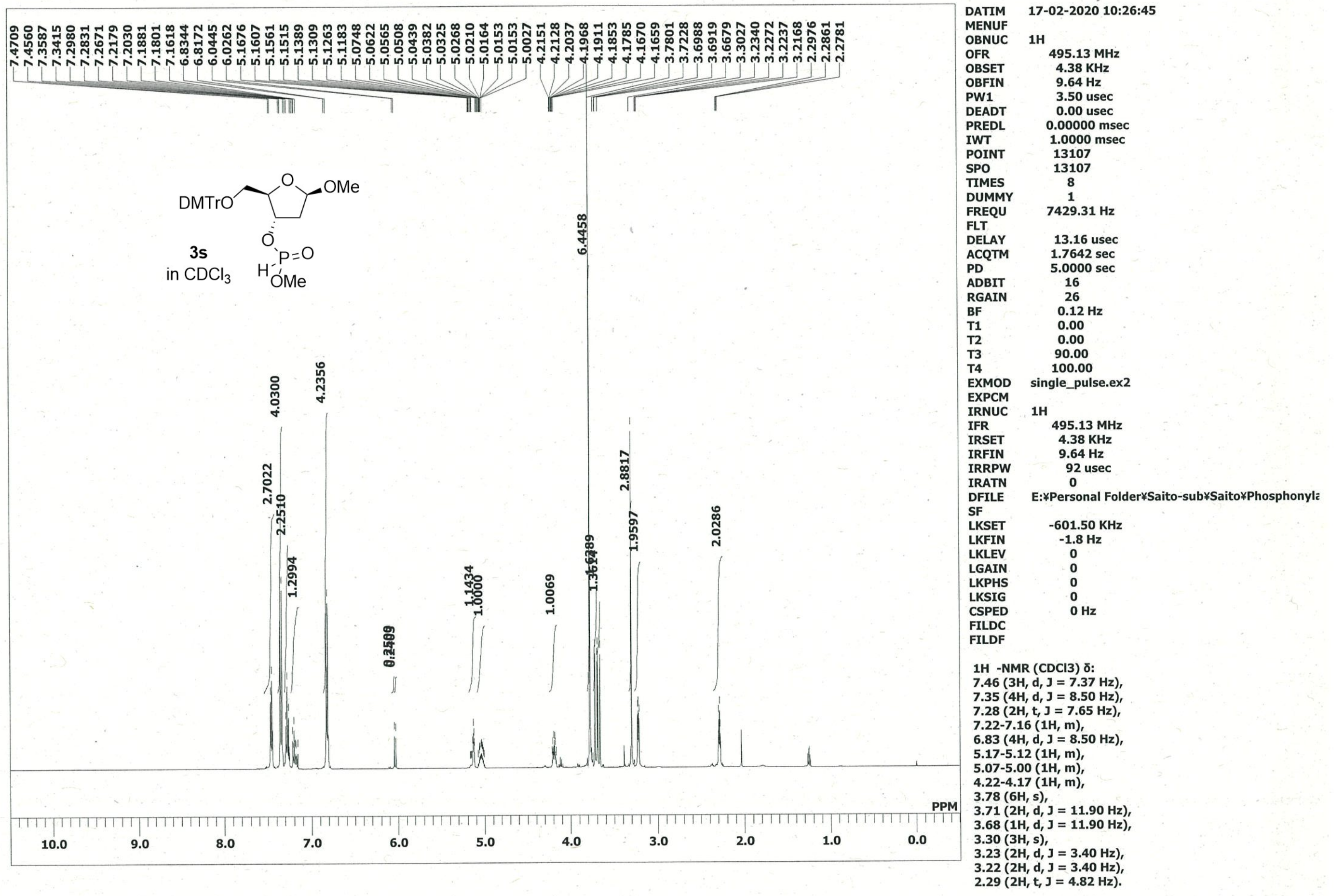




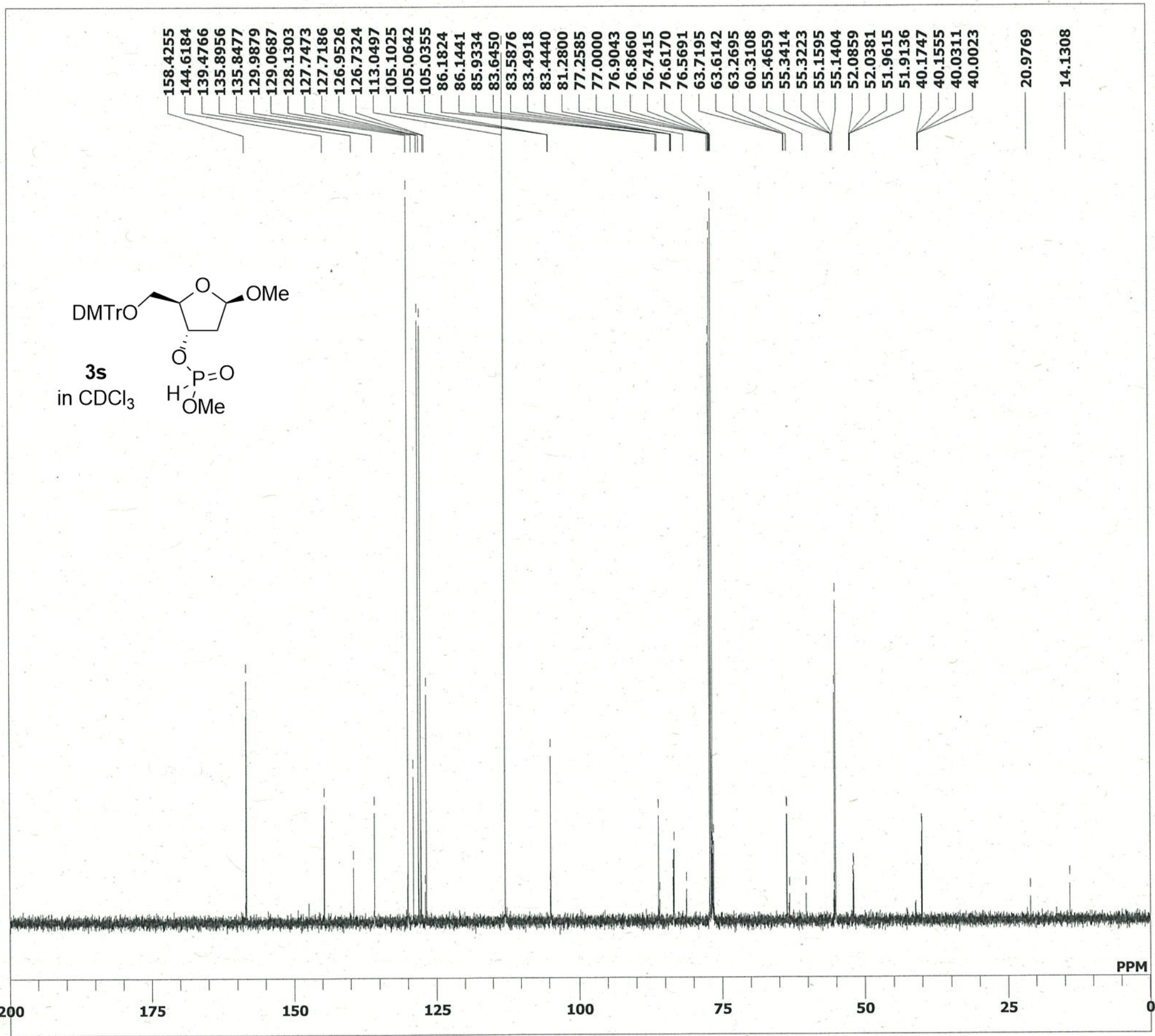

DATIM

MENUF

OFR

OBSE

OBFI

DEADT

DEADT

IWT

SPO

TIMES

DUMMY

FREQU

FLT

DELAY

ACQTM

ADBIT

RGAIN

T1

T2

T4

EXMOD

EXPCM

IRNUC

IFR

IRSET

RRPW

RATN

DFILE

SF

KFIN

KLEV

GAIN

KPHS

CSPED

FILDC

ILDF
$3 c$

$124.51 \mathrm{MHz}$

$3.45 \mathrm{KHz}$

$6.00 \mathrm{~Hz}$

0.00 usec

$0.00000 \mathrm{msec}$

$1.0000 \mathrm{msec}$

$1.0000 \mathrm{~m}$
$\mathbf{2 6 2 1 4}$

26214

290

4

$31249.52 \mathrm{~Hz}$

20.80 usec

$0.8389 \mathrm{sec}$

$2.0000 \mathrm{sec}$

16

$0.12 \mathrm{~Hz}$

0.00

0.00

90.00

100.00

ingle_pulse_dec

1H

$495.13 \mathrm{MH}$

$4.38 \mathrm{KHz}$

92 usec

E:*Personal Folder¥Saito-sub¥Saito¥Phosphonyl

$-601.50 \mathrm{KHz}$

$-1.8 \mathrm{~Hz}$

0

0

0

$\mathbf{0 ~ H z}$ 


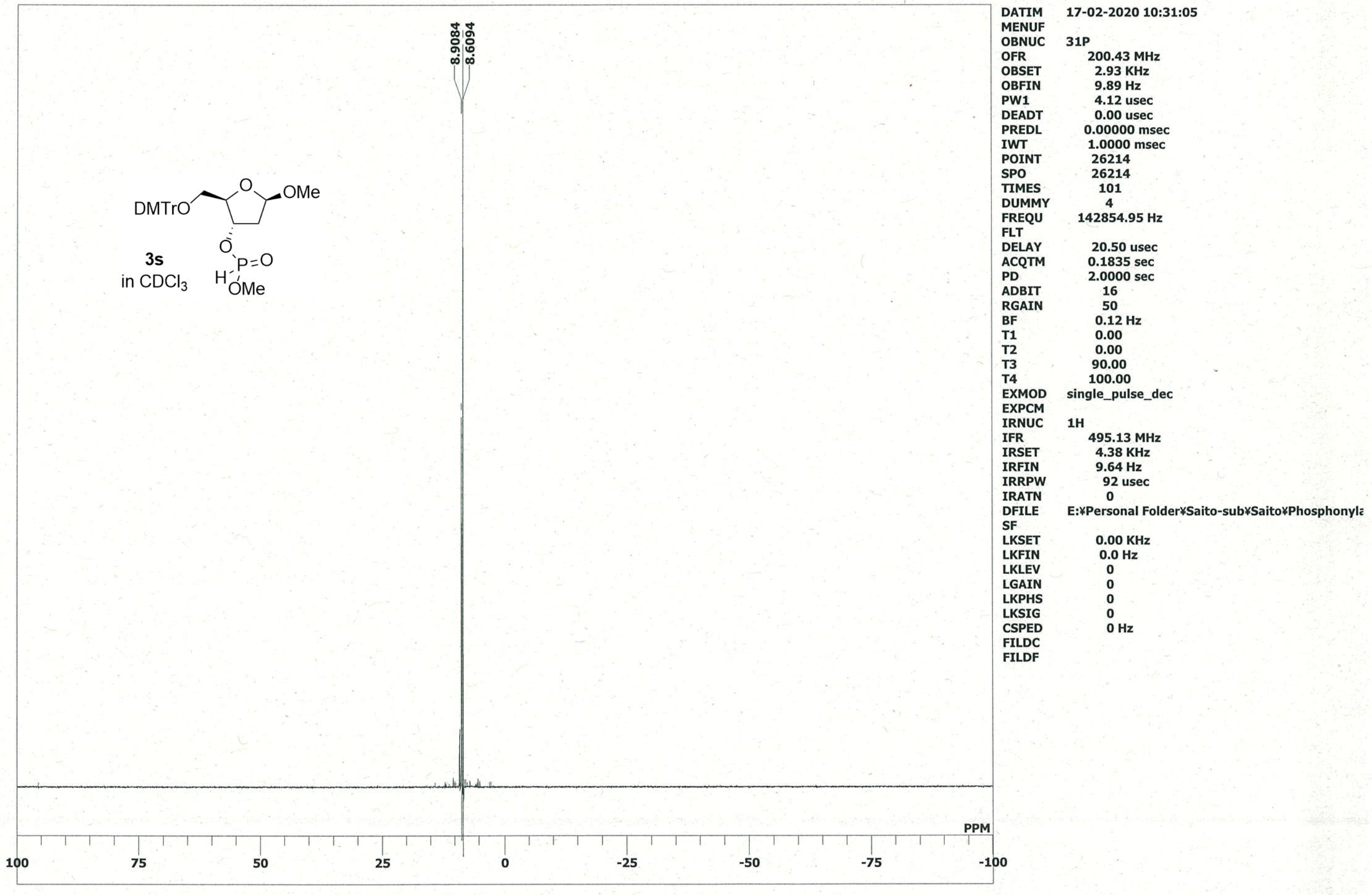




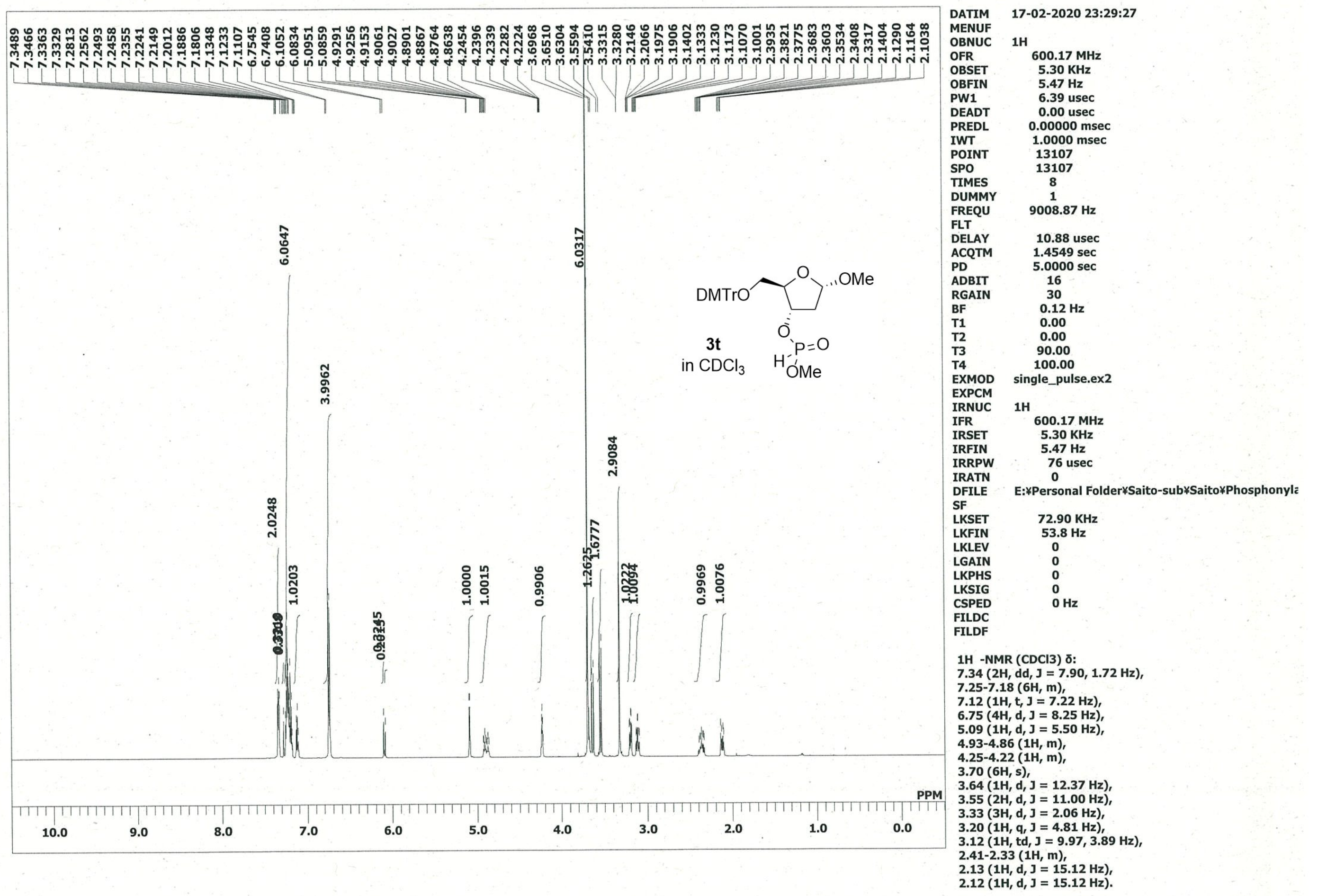


E:*Personal Folder*Saito-sub*Saito*Phosphonylation*5-DMTr-a-deoxyrib-13C.als single pulse decoupled gated NOE

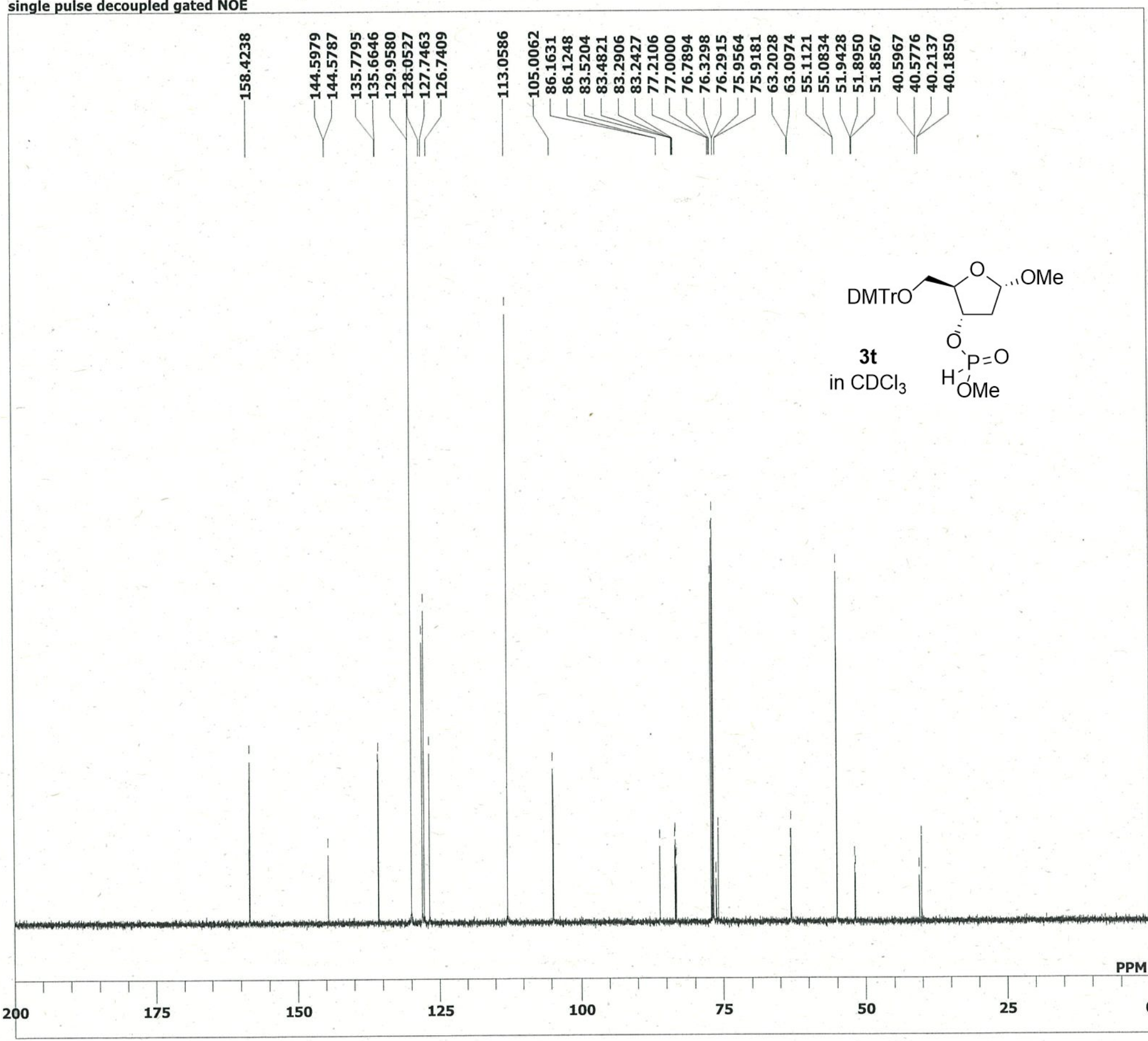

DATIM

OBNUC $13 C$

$\quad 150.92 \mathrm{MHz}$

OBSET $\quad 8.52 \mathrm{KHz}$

$\begin{array}{ll} & 1.74 \mathrm{~Hz} \\ \text { PW1 } & 4.02 \mathrm{usec}\end{array}$

$\begin{array}{ll} & 4.02 \text { usec } \\ \text { DEADT } & 0.00 \text { usec }\end{array}$

PREDL $\quad 0.00000 \mathrm{msec}$

IWT $\quad \mathbf{1 . 0 0 0 0 \mathrm { msec }}$

POINT $\quad 26214$

$\begin{array}{ll}\text { SPO } & 26214 \\ & 26214\end{array}$

TIMES $\quad 310$

DUMMY

FREQU $\quad 37878.21 \mathrm{~Hz}$

FLT

ACQTM

ACQTM
PD

PD

ADBIT

BF

BF

T1

$\begin{array}{lc}\text { T4 } & 100.00 \\ \text { EXMOD } & \text { single_pulse_dec }\end{array}$

EXPCM

IRNUC

IFR

IRSET

IRFIN

IRRPW

DFILE

SF

LKSET

LKFIN

LGAIN

LKPHS

LKSIG

CSPED

FILDC

FILDF

\subsection{2 usec
$0.6921 ~ s e c$}

$2.0000 \mathrm{sec}$

16

$0.12 \mathrm{~Hz}$

0.00

0.00

$00.17 \mathrm{MHz}$

$5.30 \mathrm{KHz}$

$76 \mathrm{usec}$

$72.90 \mathrm{KHz}$

$53.8 \mathrm{~Hz}$

0

$0 \mathrm{~Hz}$

E:¥Personal Folder¥Saito-sub¥Saito¥Phosphony 
E:*Personal Folder*Saito-sub*Saito*Phosphonylation*5-DMTr-a-deoxyrib-31P.als

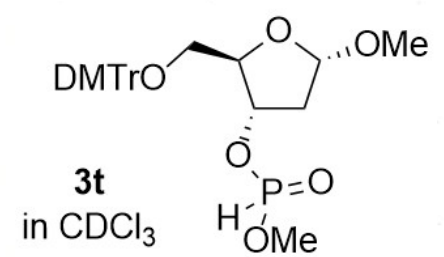

DATIM

MENUF

OBNUC

OFR

OBSET

OBFI

PW1

DEADT

PREDL

IWT

POINT

SPO

TIMES

DUMMY

FREQU

FLT

DELAY

PD

ADBIT

RGAIN

BF

B1

T2

T3

EXMOD

EXMOD

EXPCM

IRNUC

IFR

IRSET

IRFIN
IRRPW

IRRPW

IRATN

DFIL

SF

LKSET

LKFIN

LKLAIN

LKAIN

LKSIG

CSPED

FILDC

FILDC

1H
7-02-2020 23:35:12

242.95 MHz $4.04 \mathrm{KHz}$

4.00 usec

.

$.00000 \mathrm{msec}$

124

$172411.16 \mathrm{~Hz}$

20.50 usec

$2.0000 \mathrm{sec}$

16

50

$0.12 \mathrm{~Hz}$

0.00

0.00

90.00

single_pulse_dec

$600.17 \mathrm{MHz}$

$5.30 \mathrm{KHz}$

$76 \mathrm{~Hz}$

6 usec

:*Personal Folder*Saito-sub*Saito*Phosphonyla

$0.00 \mathrm{KHz}$

$0.0 \mathrm{~Hz}$

0

$\mathrm{OHz}$ 


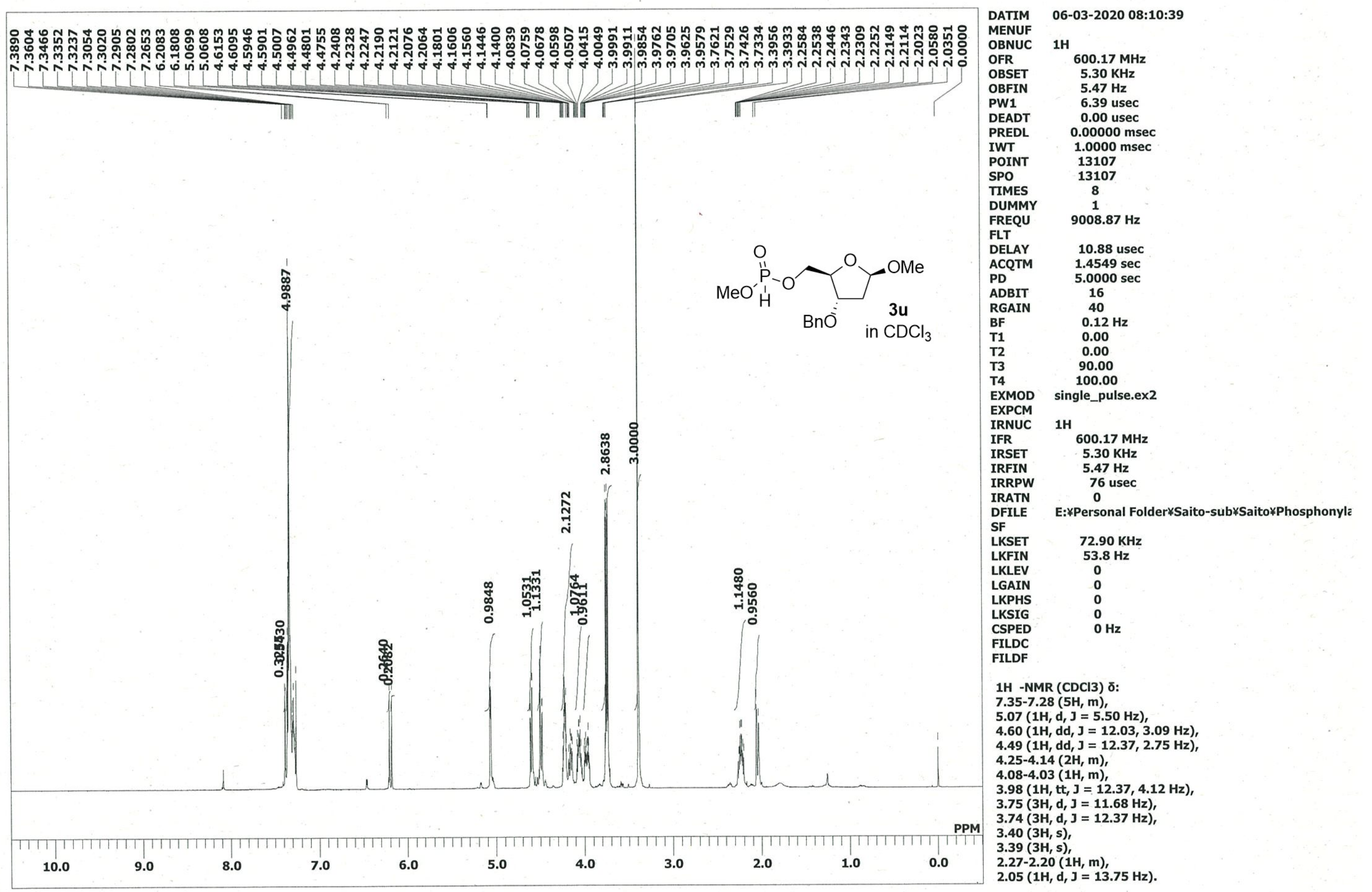


E:*Personal Folder*Saito-sub*Saito*Phosphonylation*3- Bn-b-deoxyrib-31C.als single pulse decoupled gated NOE

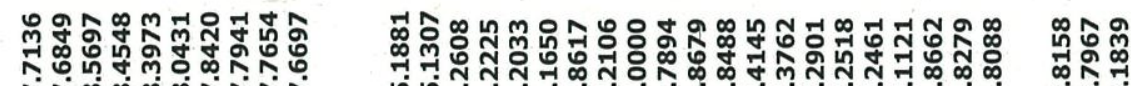 \\ คำ}

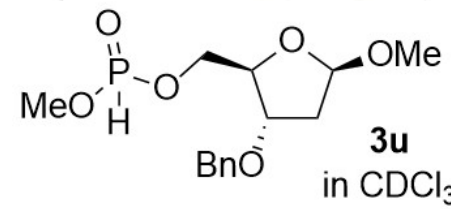

DATIM

MENUF

OBNUC

OFR

OBSE

OBFIN

PW1

DEADT

PREDL

IWT

SPO

TIMES

DUMMY

FREQU

FLT

ACOTM

ACQTM

ADBIT

RGAIN

BF

T1

T2

T4

EXMOD

EXPCM

IRNUC

IFR

IRSET

IRFIN

IRRPW

IRATN

DFILE

SF

LKSET

LKFIN

LKLEV

GAIN

KPHS

KSIG

CSPED

FILDC

FILDF
6-03-2020 08:42:07

$150.92 \mathrm{MHz}$

$8.52 \mathrm{KHz}$

$1.74 \mathrm{~Hz}$

0.00 usec

$0.00000 \mathrm{msec}$

$1.0000 \mathrm{msec}$

$\mathbf{2 6 2 1 4}$

26214

573

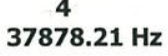

21.12 usec

$0.6921 \mathrm{sec}$

$2.0000 \mathrm{sec}$

16

$0.12 \mathrm{~Hz}$

0.00

90.00

100.00

1H

$600.17 \mathrm{MHz}$

$5.30 \mathrm{KHz}$
$5.47 \mathrm{~Hz}$

76 usec

E:ҰPersonal Folder¥Saito-sub¥Saito¥Phosphonyla

$72.90 \mathrm{KHz}$

$3.8 \mathrm{~Hz}$

0

0

$\mathrm{OHz}$ 


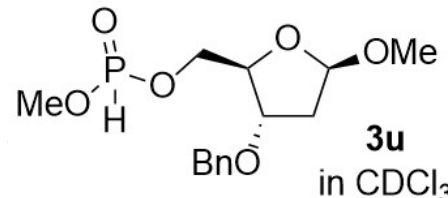

DATIM

OBNUC

OF

OBSET

OBFIN

PW1

DEADT

PRED

IWT

POIN

SPO

TIMES

DUMMY

FLT

DELAY

ACQTM

PD

ADBIT

BF

T1

12

T3

EXMOD

EXPCM

IRNUC

IFR

IRSET

IRFIN

IRRPW

DFILE

DFF

LKSET

LKFIN

LKLEV

LGAIN

LKPHS

LKSIG

CSPED

FILDC

FILDF 
DATIM

MENUF

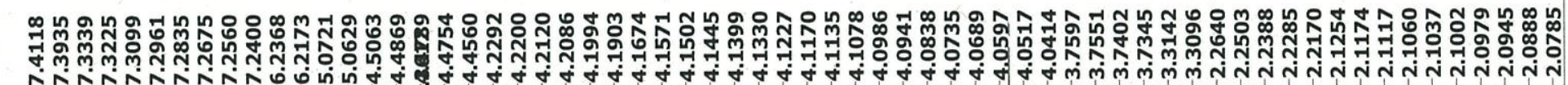

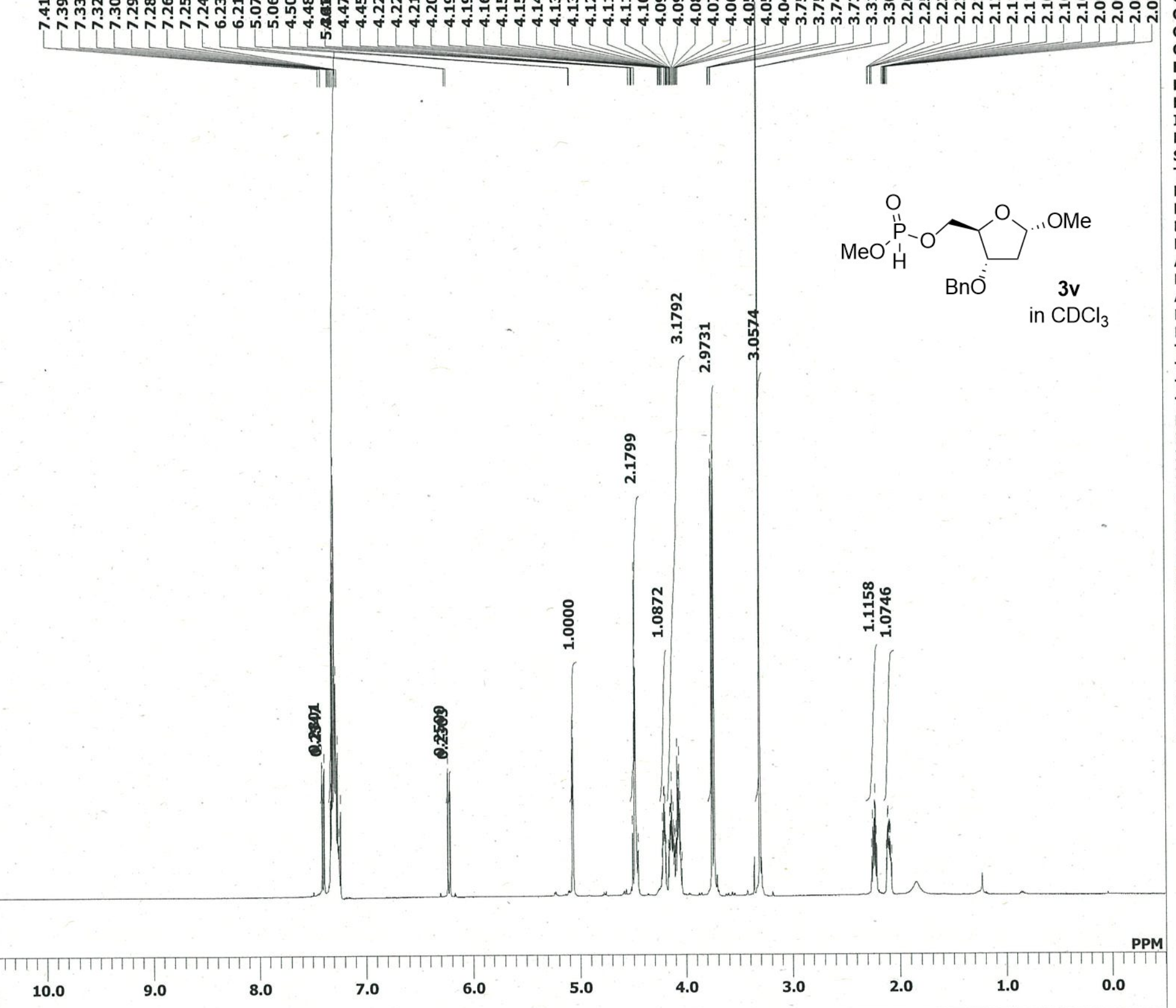

04-03-2020 04:24:32 $1 \mathrm{H}$

$600.17 \mathrm{MHz}$

OBSET $\quad 5.30 \mathrm{KHz}$

PW1 $\quad 6.39$ usec

DEADT $\quad 0.00$ usec

PREDL $\quad 0.00000 \mathrm{msec}$

IWT $\quad 1.0000 \mathrm{msec}$

\begin{tabular}{ll}
13107 \\
\hline OINT & 13107
\end{tabular}

TIMES $\quad 8$

$\begin{array}{lc}1 \\ \text { DUMMY } & 1 \\ 9008.87 \mathrm{~Hz}\end{array}$

DELAY

ACQTM

PD

ADBIT

RGAIN

BF

BF

T2

T4

EXMOD single_pulse.ex2

EXPCM

IRNUC

IFR

1H

10.88 usec

$1.4549 \mathrm{sec}$

$5.0000 \mathrm{sec}$

16

$0.12 \mathrm{~Hz}$

0.00

0.00

90.00

IRSET

IRFIN

$600.17 \mathrm{MHz}$

$5.30 \mathrm{KHz}$

76 usec

E:*Personal Folder*Saito-sub*Saito*Phosphonyl

LKSET $\quad 72.90 \mathrm{KHz}$

LKFIN $\quad 53.8 \mathrm{~Hz}$

LKLEV 0

LGAIN 0

LKSIG 0

CSPED $\quad 0 \mathrm{~Hz}$

1H -NMR ( $\mathrm{CDCl} 3)$ ס:

7.33-7.26 $(5 \mathrm{H}, \mathrm{m})$,

$5.07(1 \mathrm{H}, \mathrm{d}, \mathrm{J}=5.50 \mathrm{~Hz}$,

4.51-4.46 $(2 \mathrm{H}, \mathrm{m})$,

4.23-4.19 (1H, m),

4.17-4.04 (3H, $\mathrm{m})$

$3.76(3 \mathrm{H}, \mathrm{d}, \mathrm{J}=2.75 \mathrm{~Hz})$

$3.74(3 \mathrm{H}, \mathrm{d}, \mathrm{J}=3.44 \mathrm{~Hz})$,

2.26-2.22 (1H, m), 
E:*Personal Folder*Saito-sub*Saito*Phosphonylation*3- Bn-a-deoxyrib-13C.als single pulse decoupled gated NOE
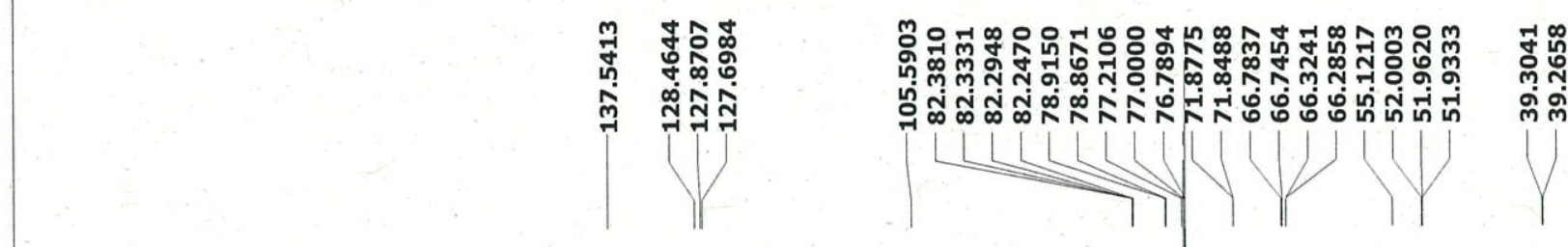

DATIM

04-03-2020 04:49:15

MENUF

OBNUC $13 \mathrm{C}$

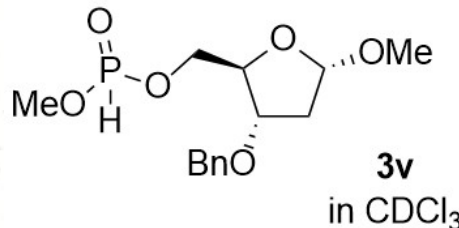

OFR

OBSET

OBFIN

PW1

DEADT

PRED

IWT

POINT

SPO

TIMES

DUMMY

FLT

DELAY

DELAY

PDQ

ADBIT

RGAIN

BF

T1

T2

$150.92 \mathrm{MHz}$

$8.52 \mathrm{KHz}$

$1.74 \mathrm{~Hz}$

4.02 usec

$0.00000 \mathrm{msec}$

$1.0000 \mathrm{msec}$

26214

26214

431

4
37878.21

21.12 usec

$0.6921 \mathrm{sec}$

$2.0000 \mathrm{sec}$

16

$0.12 \mathrm{~Hz}$

0.00

9.00

100.00

EXMOD single_pulse_dec

EXPCM

IRNUC

IRN

1H

IRSET

$600.17 \mathrm{MHz}$

IRFIN

$5.30 \mathrm{KHz}$

IRATN

DFILE E:*Personal Folder*Saito-sub*Saito*Phosphonyla

LKSE

$72.90 \mathrm{KHz}$

$53.8 \mathrm{~Hz}$

LKLEV $\quad 0$

KAIN

$\begin{array}{ll}\text { LKSIG } & 0 \\ \text { CSPED } & 0\end{array}$

FILDF

0
0
0
0 
E:*Personal Folder*Saito-sub*Saito*Phosphonylation*3- Bn-a-deoxyrib-31P.als

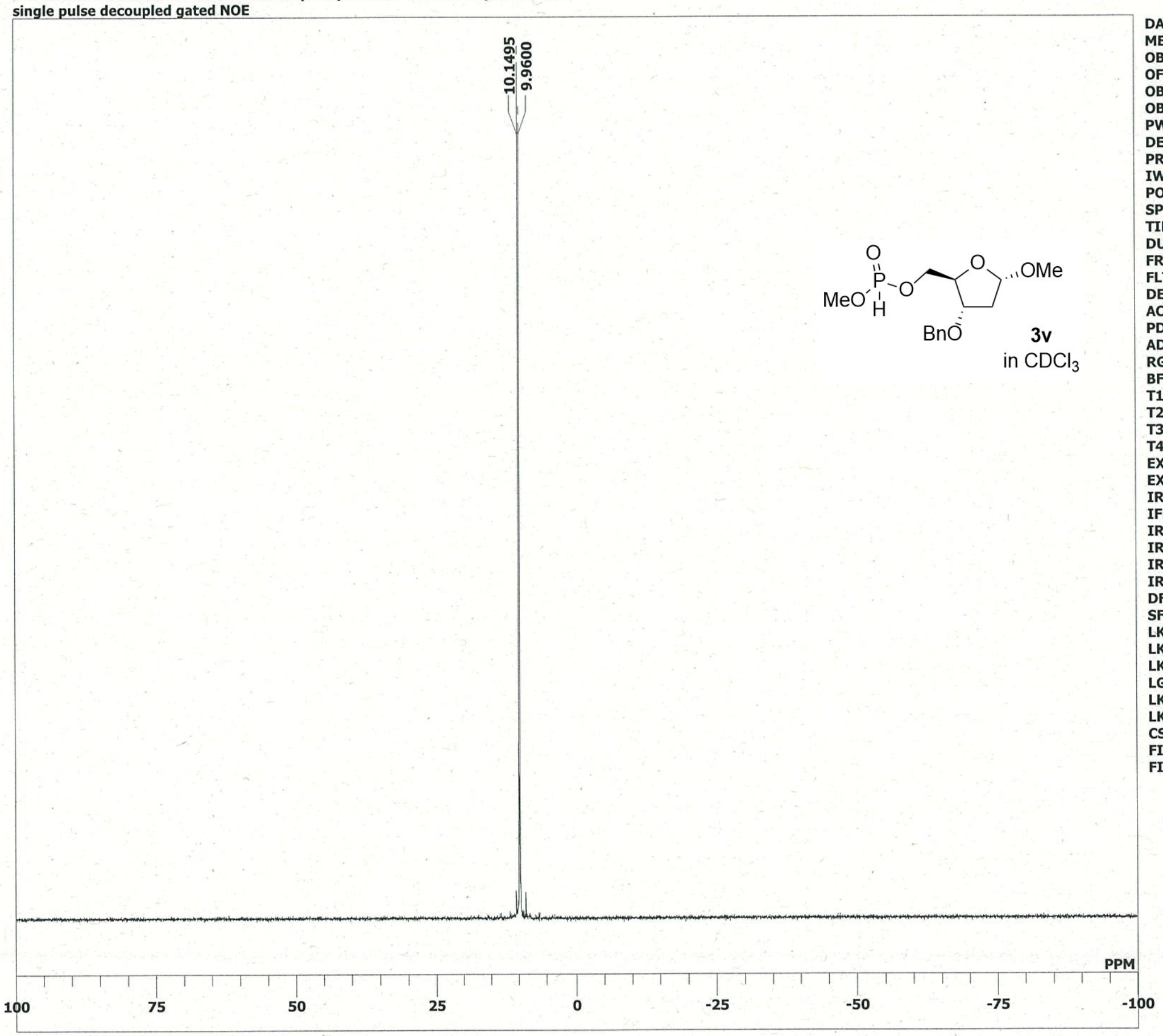

04-03-2020 04:28:29 $31 P$

$242.95 \mathrm{MHz}$ $4.04 \mathrm{KHz}$ $1.25 \mathrm{~Hz}$

4.00 usec

$0.00000 \mathrm{msec}$

$1.0000 \mathrm{msec}$

26214

\begin{tabular}{ll} 
PPO & 26214 \\
\hline & 26214
\end{tabular}

TIMES $\quad 104$

$\begin{array}{lc}4 \\ \text { DUMMY } & 4 \\ & 172411.16 \mathrm{~Hz}\end{array}$

$172411.16 \mathrm{~Hz}$

DELAY 20.50 usec

$\begin{array}{ll}20.50 \mathrm{sec} \\ \text { ACQTM } & 0.1520 \mathrm{sec}\end{array}$

ADBIT $\quad 2.0000 \mathrm{sec}$

RGAIN $\quad 50$

$\begin{array}{ll}B F & 0.12 \mathrm{~Hz}\end{array}$

T1 0.00

$\begin{array}{ll}\mathrm{T} 2 & 0.00 \\ \mathrm{~T} & 90.00\end{array}$

$\begin{array}{lc}90.00 \\ \text { T4 } & 100.00\end{array}$

EXMOD single_pulse_dec

XPCM

EXPCM

IFR

$1 \mathrm{H}$

IRFIN $\quad 5.47 \mathrm{~Hz}$

IRRPW $\quad 76$ usec

E:¥Personal Folder¥Saito-sub¥Saito¥Phosphonyle

LKSET $\quad 0.00 \mathrm{KHz}$

$0.0 \mathrm{~Hz}$

LKLEV $\quad 0$

LKPHS 0

LSSIG

CSPED

FILDF

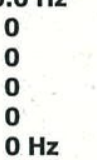




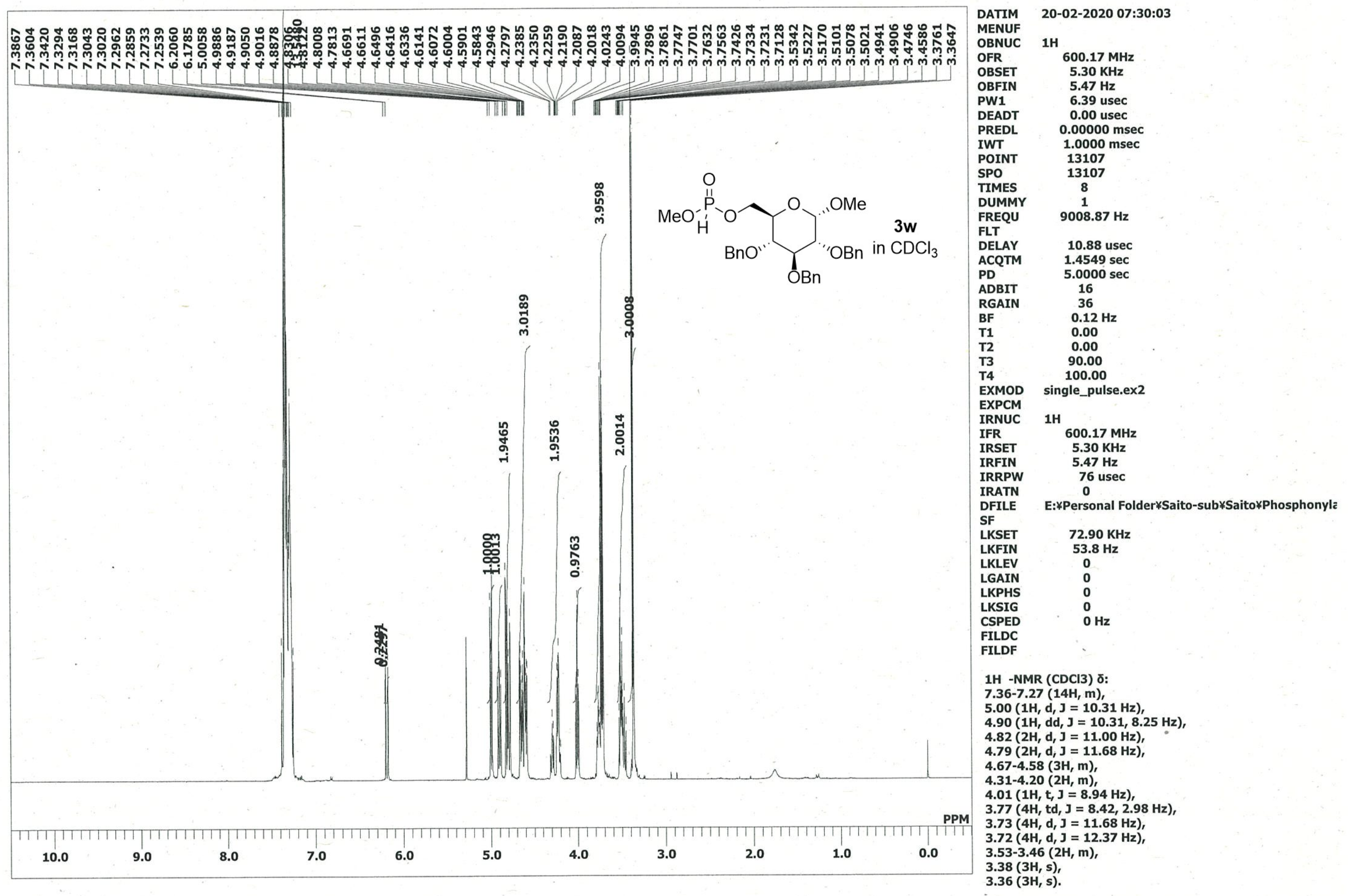




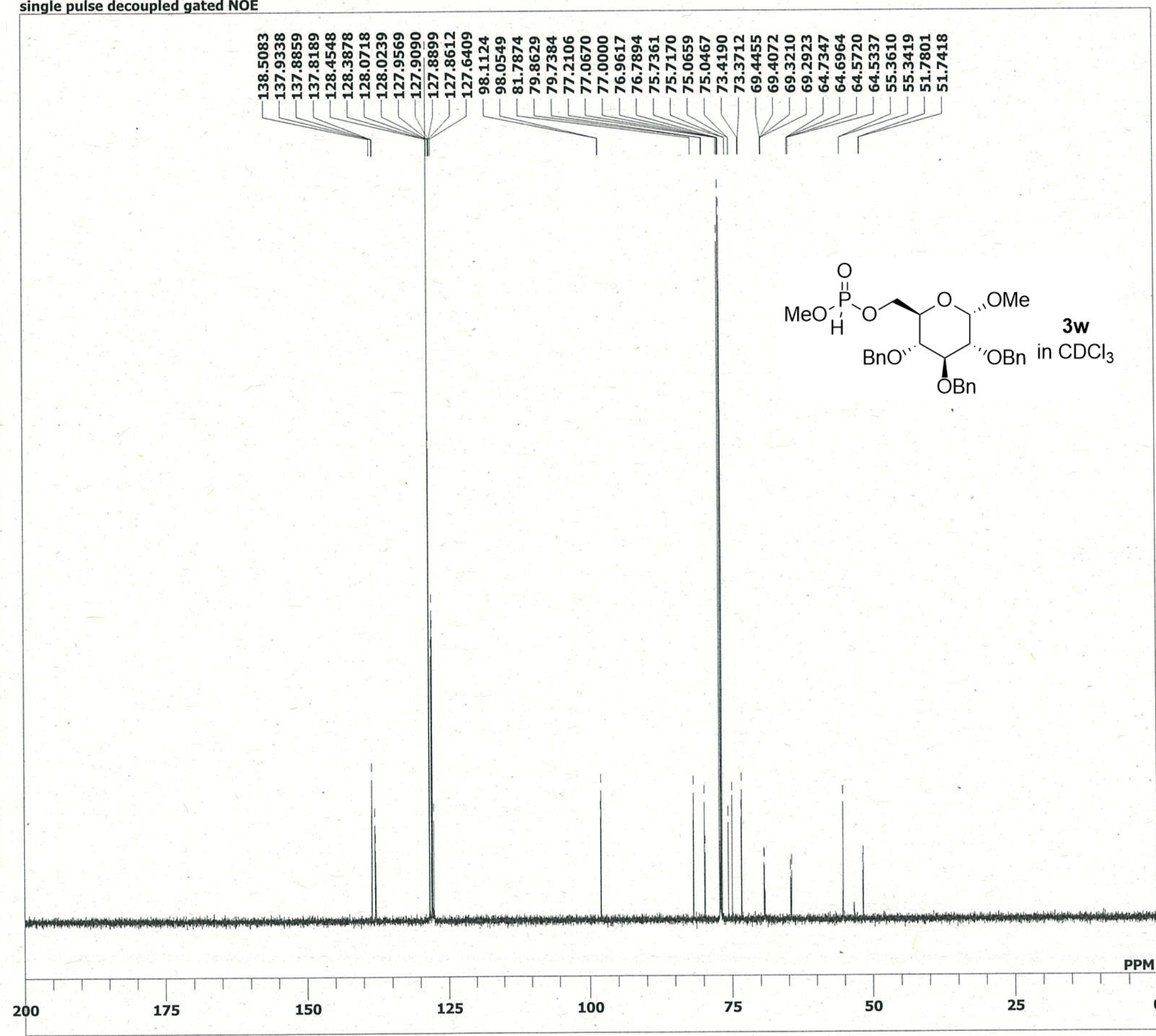

DATIM

MENUF

OBNUC 13C

$\quad 150.92 \mathrm{MHz}$

$\begin{array}{ll} & 8.52 \mathrm{KHz} \\ & \end{array}$

$8.75 \mathrm{~Hz}$
$\mathrm{OB} 1 \mathrm{~B}$

PWI $\quad 4.02$ usec

PREDI $\quad 0.00000 \mathrm{msec}$

IWT $\quad 1.0000 \mathrm{msec}$

$\begin{array}{ll} & 1.0000 \mathrm{~ms} \\ \text { POINT } & 26214\end{array}$

SPO $\quad 26214$

TIMES $\quad 26214$

TIMES

$\begin{array}{ll}4 \\ \text { FREQU } & 37878.21 \mathrm{~Hz}\end{array}$

21.12 usec

ACQTM $\quad 0.6921 \mathrm{sec}$

PD $2.0000 \mathrm{sec}$

ADBIT $\quad 16$

BF $\quad 0.12 \mathrm{~Hz}$

$\begin{array}{ll}\mathrm{T} 1 & 0.00 \\ \mathrm{~T} & 0.00\end{array}$

$\begin{array}{ll} & 0.00 \\ \mathrm{~T} 3 & 90.00\end{array}$

90.00
74 $\quad 100.00$

EXMOD single_pulse_dec

EXPCM

IRNUC

IFR

$1 \mathrm{H}$

IRSET

IRFIN

IRATN

DFILE

SF

LKSET

LKFIN

LKLEV

LAIN

LKPHS

KSIG

CSPED

FILDC

FILDF
$600.17 \mathrm{MHz}$

$5.30 \mathrm{KHz}$

76 usec

0

E:*Personal Folder*Saito-sub*Saito¥Phosphonyle

$72.90 \mathrm{KHz}$

$3.8 \mathrm{~Hz}$

0

0

$\mathrm{OHz}$ 
E:*Personal Folder*Saito-sub¥Saito $¥$ Phosphonylation $¥ 2,3,4-B n-g l u-31 P . a l s$

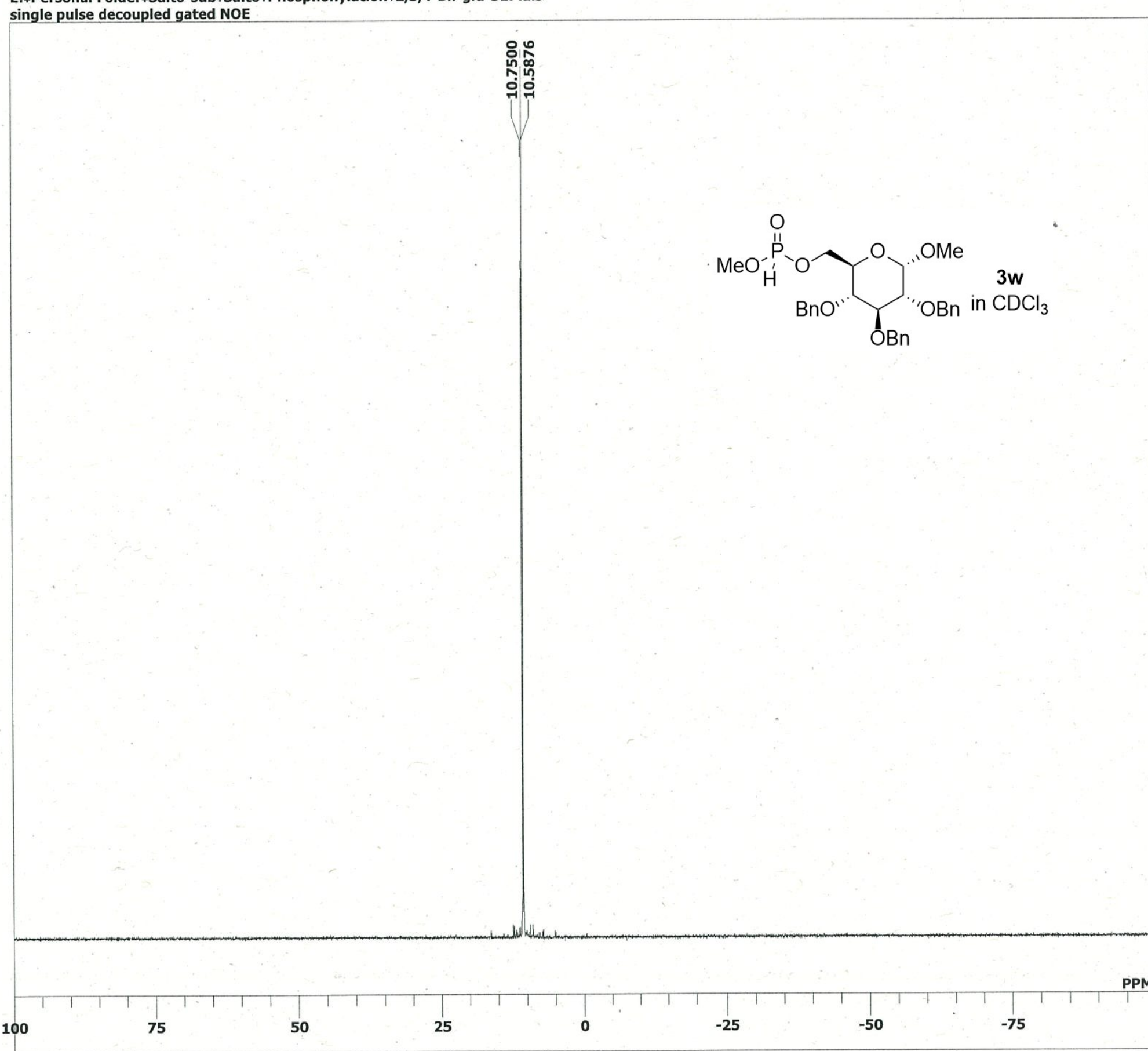

20-02-2020 07:36:02

MBNUC 31

OFR $\quad 242.95 \mathrm{MHz}$

OBSET $\quad 4.04 \mathrm{KHz}$

$\begin{array}{ll} & 1.25 \mathrm{~Hz}\end{array}$

PW1 $\quad 4.00$ usec

0.00 usec

$0.00000 \mathrm{mse}$

IWT $\quad 1.0000 \mathrm{msec}$

\begin{tabular}{ll}
26214 \\
\hline
\end{tabular}

TIMES $\quad 26214$

$\quad 131$

FREQU $\quad 172411.16 \mathrm{~Hz}$

DELAY 20.50 usec

ACQTM $\quad 0.1520 \mathrm{sec}$

PD $\quad 2.0000 \mathrm{sec}$

$\begin{array}{lll}\text { ADBIT } & 16 \\ \text { RGAIN } & 50\end{array}$

$\begin{array}{ll}\text { BF } & 50 \\ \text { T } & 0.12 \mathrm{~Hz}\end{array}$

T1 0.00

T3 $\quad 9.00$

T4 100.00

EXMOD single_pulse_dec

EXPCM

IRNUC

IFR

$5.30 \mathrm{KHz}$

IRFIN $\quad 5.47 \mathrm{~Hz}$

IRRPW $\quad 76$ usec

DFILE E:*Personal Folder*Saito-sub*Saito¥Phosphonyla

$0.00 \mathrm{KHz}$

LKLEV

LGAIN $\quad 0$

LKPHS $\quad 0$

CSPED $\quad 0 \mathrm{~Hz}$

FILDF 


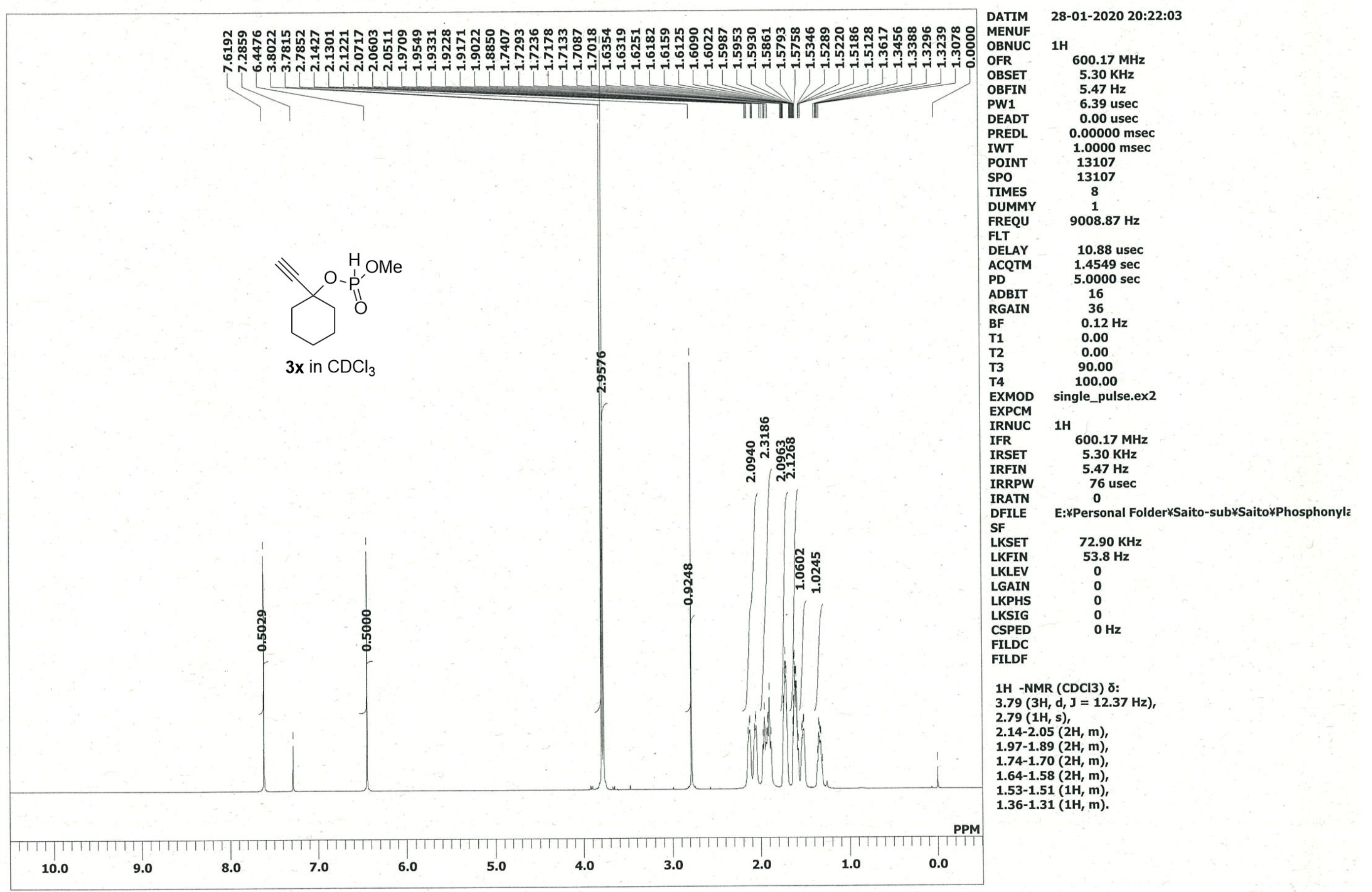


E:*Personal Folder*Saito-sub*Saito¥Phosphonylation*ethynyl-c-hex-13C.als

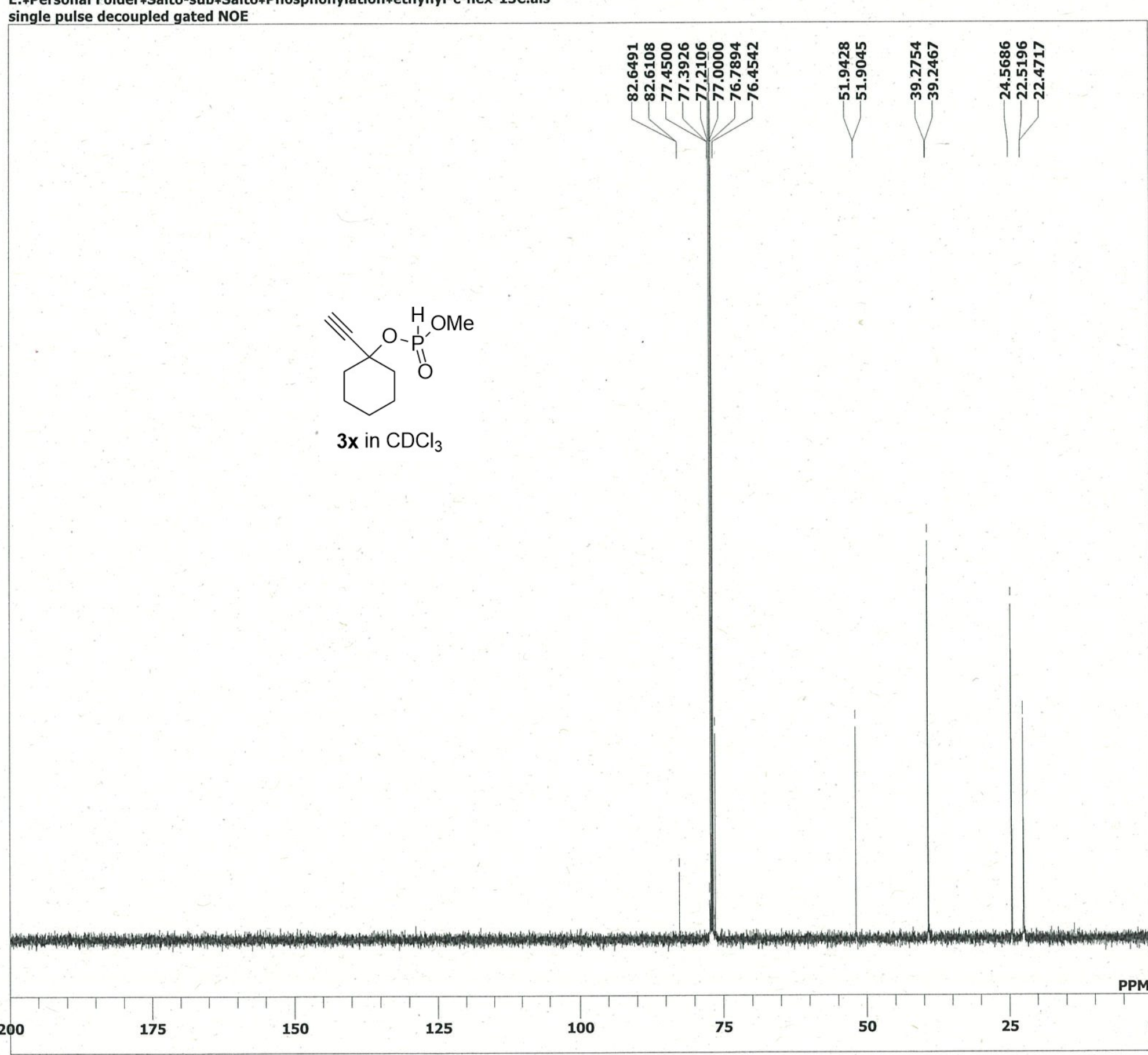

28-01-2020 20:56:57

MENUF

BNUC $13 C$

OFR $\quad 150.92 \mathrm{MHz}$

OBSET $\quad 8.52 \mathrm{KHz}$

PW1 4.02 usec

DEADT 0.00 usec

PREDL $0.00000 \mathrm{msec}$

IWT $\quad 0.00000 \mathrm{msec}$

$\begin{array}{ll}\text { POINT } & \mathbf{2 6 2 1 4}\end{array}$

SPO $\quad 26214$

TIMES 471

FREQU $\quad 37878.21 \mathrm{~Hz}$

DELAY 21.12 usec

PD $\quad 0.6921 \mathrm{sec}$

ADBIT $\quad 16$

$\begin{array}{lll}\text { RGAIN } & 0.12 \mathrm{~Hz}\end{array}$

10.12

T2 0.00

$\begin{array}{ll}\mathrm{T} 3 & \mathbf{9 0 . 0 0}\end{array}$

T4 $\quad 100.00$

EXMOD single_pulse_dec

EXPCM

$\quad 600.17 \mathrm{MHz}$

IRFIN $\quad 5.30 \mathrm{KHz}$

IRRPW $\quad 5.47 \mathrm{~Hz}$

IRATN 0

EFILE

SF

E:¥Personal Fo

$\quad 72.90 \mathrm{KHz}$

LKFIN $\quad 53.8 \mathrm{~Hz}$

LKLEV 0

LKPHS 0

LKSIG 0

FILDF

$13 \mathrm{C}$-NMR ( $\mathrm{CDCl} 3)$ ठ:

$82.63(\mathrm{OH}, \mathrm{d}, \mathrm{J}=5.78 \mathrm{~Hz})$,

$77.42(\mathrm{OH}, \mathrm{d}, \mathrm{J}=\mathbf{8 . 6 7 \mathrm { Hz } )}$

$76.45(\mathrm{OH}, \mathrm{s})$

$51.92(\mathrm{OH}, \mathrm{d}, \mathrm{J}=5.78 \mathrm{~Hz})$

$39.26(\mathrm{OH}, \mathrm{d}, \mathrm{J}=4.33 \mathrm{~Hz})$

$24.57(\mathrm{OH}, \mathrm{s})$,

$22.50(\mathrm{OH}, \mathrm{d}, \mathrm{J}=7.22 \mathrm{~Hz})$ 
28-01-2020 20:34:16

MENUF

OBNUC 31

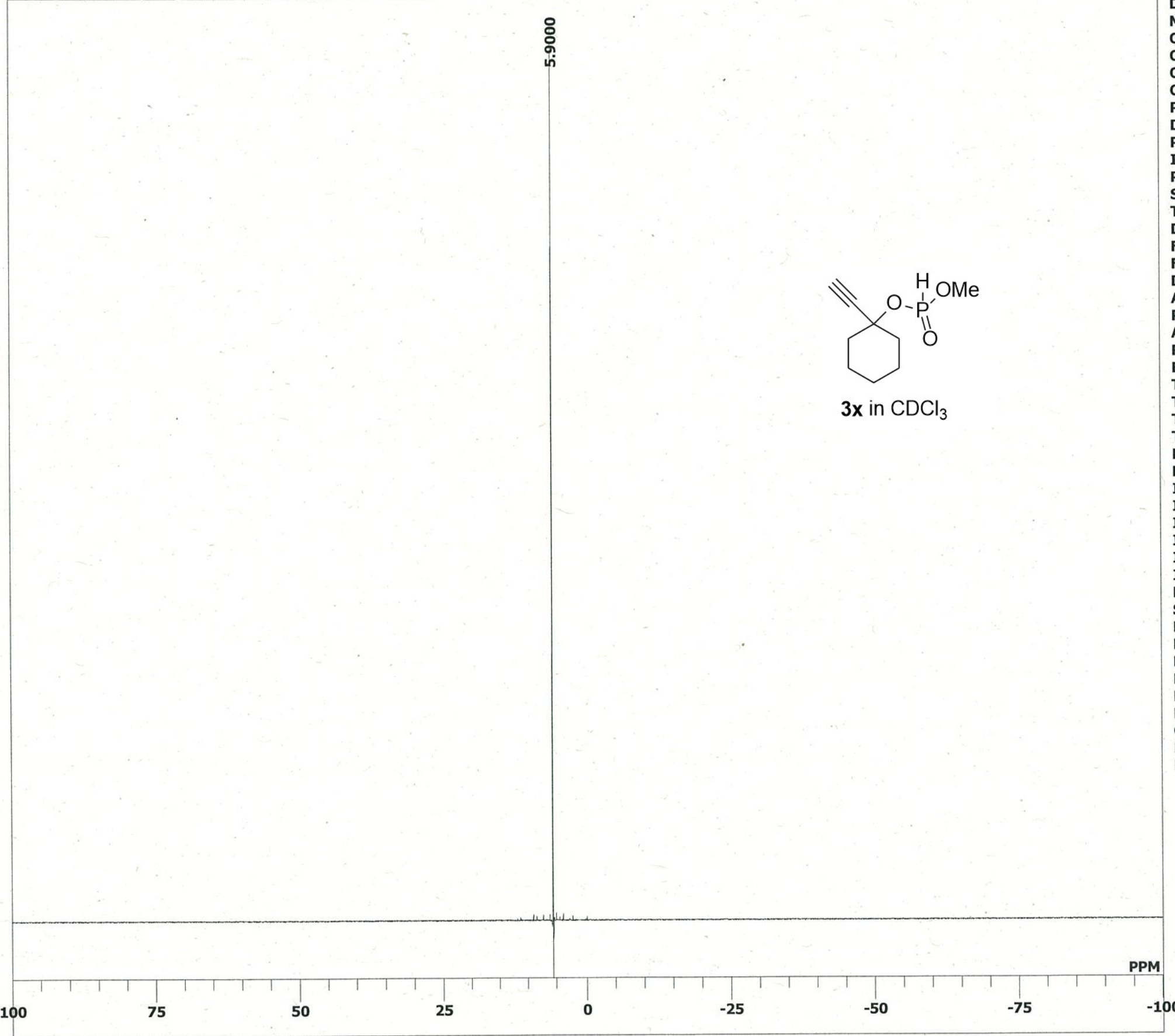

242.95 MHz

$4.04 \mathrm{KHz}$

$0.25 \mathrm{~Hz}$
$\mathrm{OB} 1$

P.00 usec

PREDL $\quad 0.00$ usec

IWT $\quad 0.00000 \mathrm{msec}$

TIMES $\quad 39$

FREQU $\quad 172411.16 \mathrm{~Hz}$

DELAY 20.50 usec

ACQTM $\quad 0.1520 \mathrm{sec}$

ADBIT $\quad 16$

BF $\quad 0.12 \mathrm{~Hz}$

T1 0.00

T2 0.00

$\begin{array}{ll}7 & 90.00 \\ 7 & 100.00\end{array}$

EXMOD single_pulse_dec

EXPCM $1 \mathrm{H}$

促

IRSE

IRFIN

IRRPW

IRATN

DFILE

SF

KSET

LKFIN

LKLEV

GAIN

LKPHS

LKSIG

CSPED $\begin{array}{ll} & 1.0000 \mathrm{~m} \\ \text { POINT } & 26214\end{array}$

SPO 26214
$600.17 \mathrm{MHz}$

$5.30 \mathrm{KHz}$

$5.47 \mathrm{~Hz}$

E:¥Personal Folder¥Saito-sub¥Saito¥Phosphonyla

$0.00 \mathrm{KHz}$

$0.0 \mathrm{~Hz}$

$\mathrm{OHz}$ 
¥*Eca\#data*Cho*expt668-isolate-1.jdf

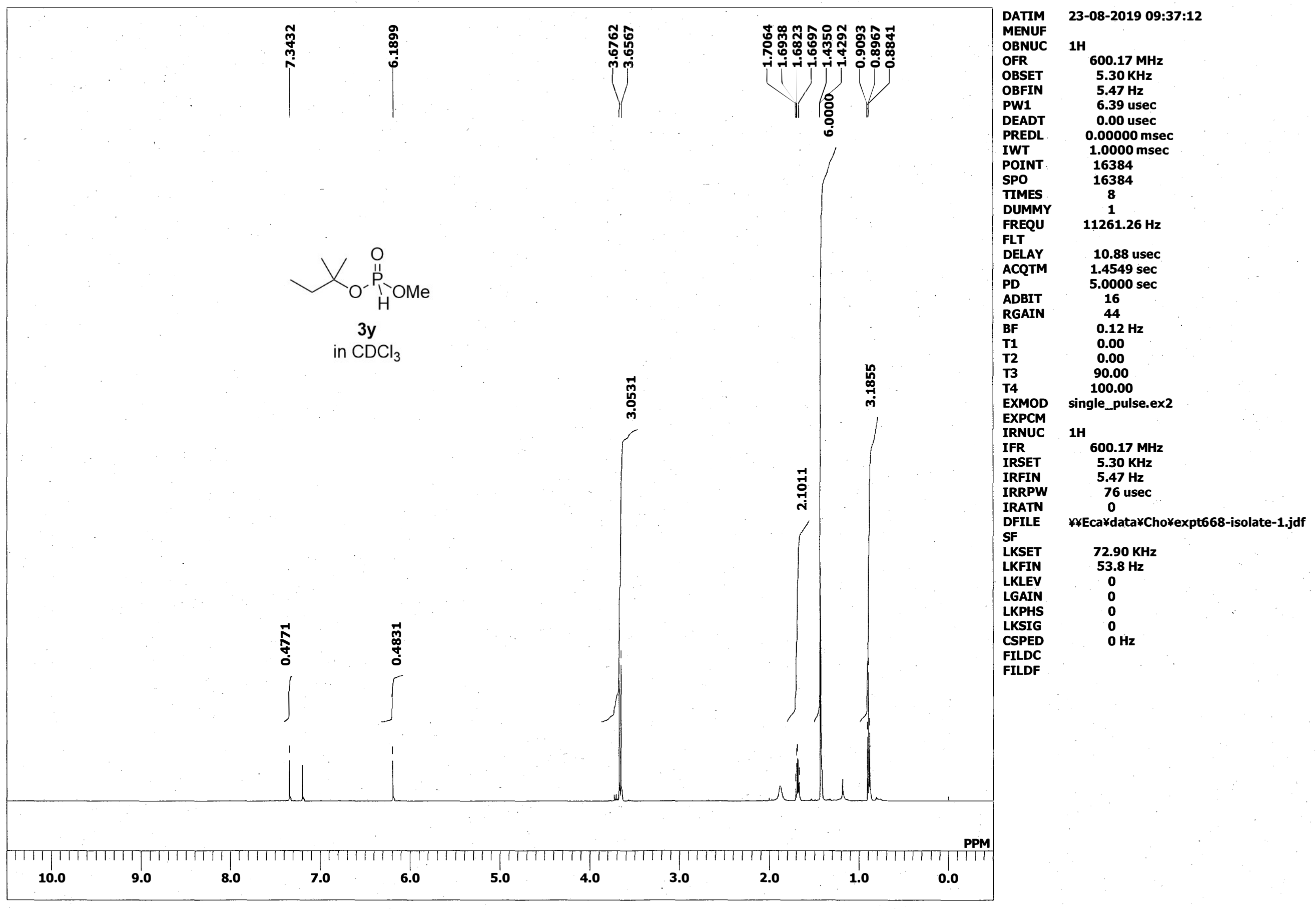




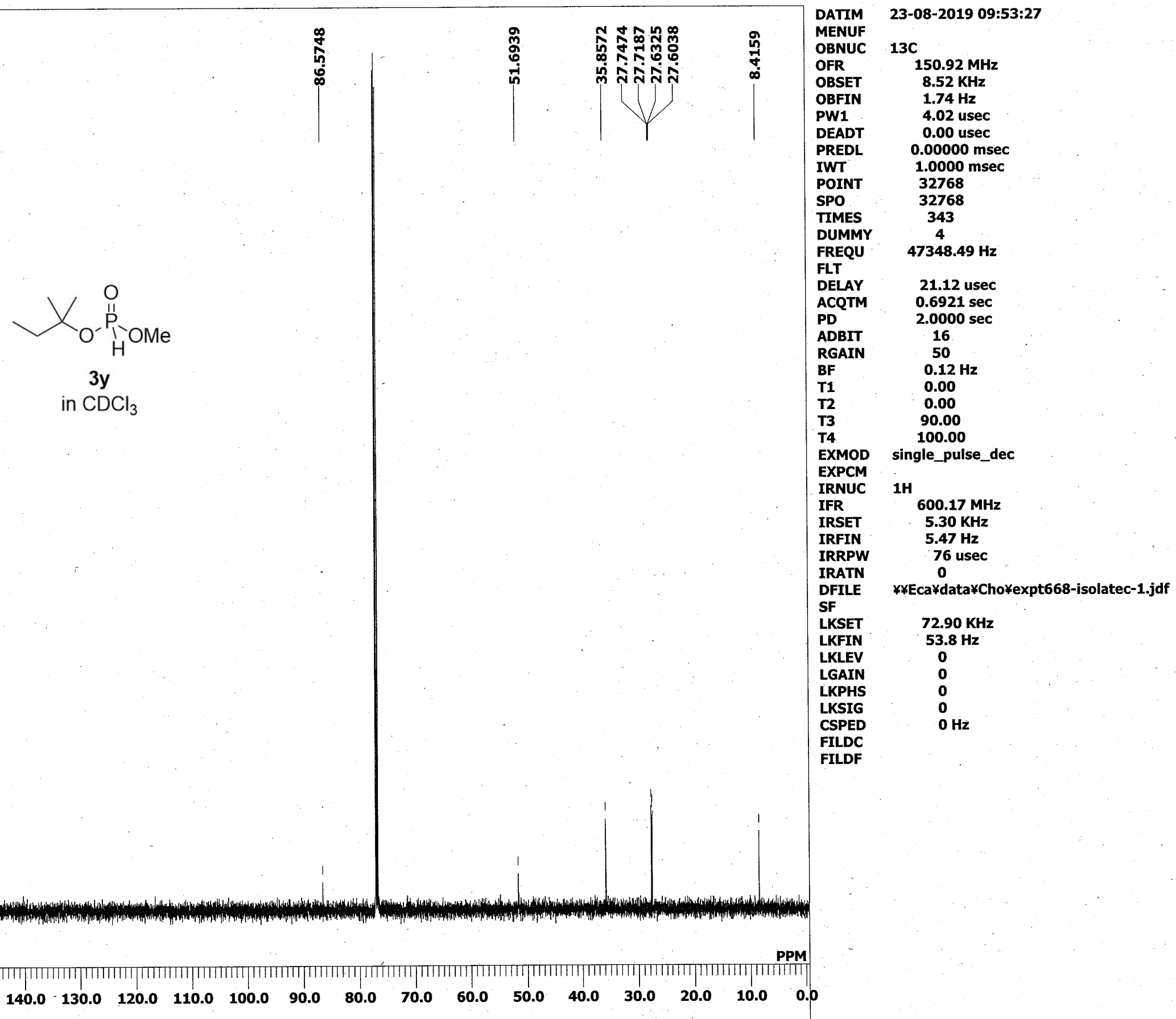


23-08-2019 09:58:19

$\begin{array}{ll}\text { MFR } & \text { 242.95 MHz }\end{array}$

OBSET $\quad 4.04 \mathrm{KHz}$

OBFIN $\quad 1.25 \mathrm{~Hz}$

PWI 4.00 usec

$\begin{array}{ll}\text { PREDL } & 0.00 \text { usec } \\ \text { PWI } & 0.00000 \mathrm{msec}\end{array}$

IWT $\quad 1.0000 \mathrm{msec}$

$\begin{array}{ll}\text { PWT } & 1.0000 \mathrm{~m} \\ \text { POINT } & 32768\end{array}$

$\begin{array}{ll}\text { SPO } & 32768\end{array}$

TIMES $\quad 85$

$\begin{array}{lc}\text { DUMMY } & 4 \\ \text { TREQU } & 215517.23\end{array}$

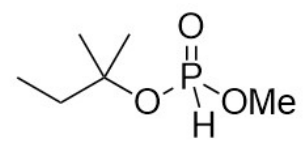

FLT

DELAY 20.50 usec

ACQTM $0.1520 \mathrm{sec}$

\begin{tabular}{ll} 
ADBIT & 2.0000 \\
& 16 \\
\hline & 50
\end{tabular}

3y

in $\mathrm{CDCl}_{3}$

BF

T1

T3

$14 \quad 100.00$

Single_pulse_dec

EXPCM

IRNUC

1H

$\begin{array}{ll} & \\ \text { IRFIN } & 5.30 \mathrm{KHz} \\ & \end{array}$

$5.47 \mathrm{~Hz}$
IRRPW $\quad 76$ usec

IRATN 0

DFILE \#\#Eca*data\#Cho¥expt668-isolatep-1.jdf

$0.00 \mathrm{KHz}$

$\quad 0.00 \mathrm{KHz}$

LKFIN $\quad 0.0$

LGAIN

LKPHS 0

$\begin{array}{ll}\text { LKSIG } & 0 \\ \text { CSPED } & 0\end{array}$

FILDC

FIIDF 


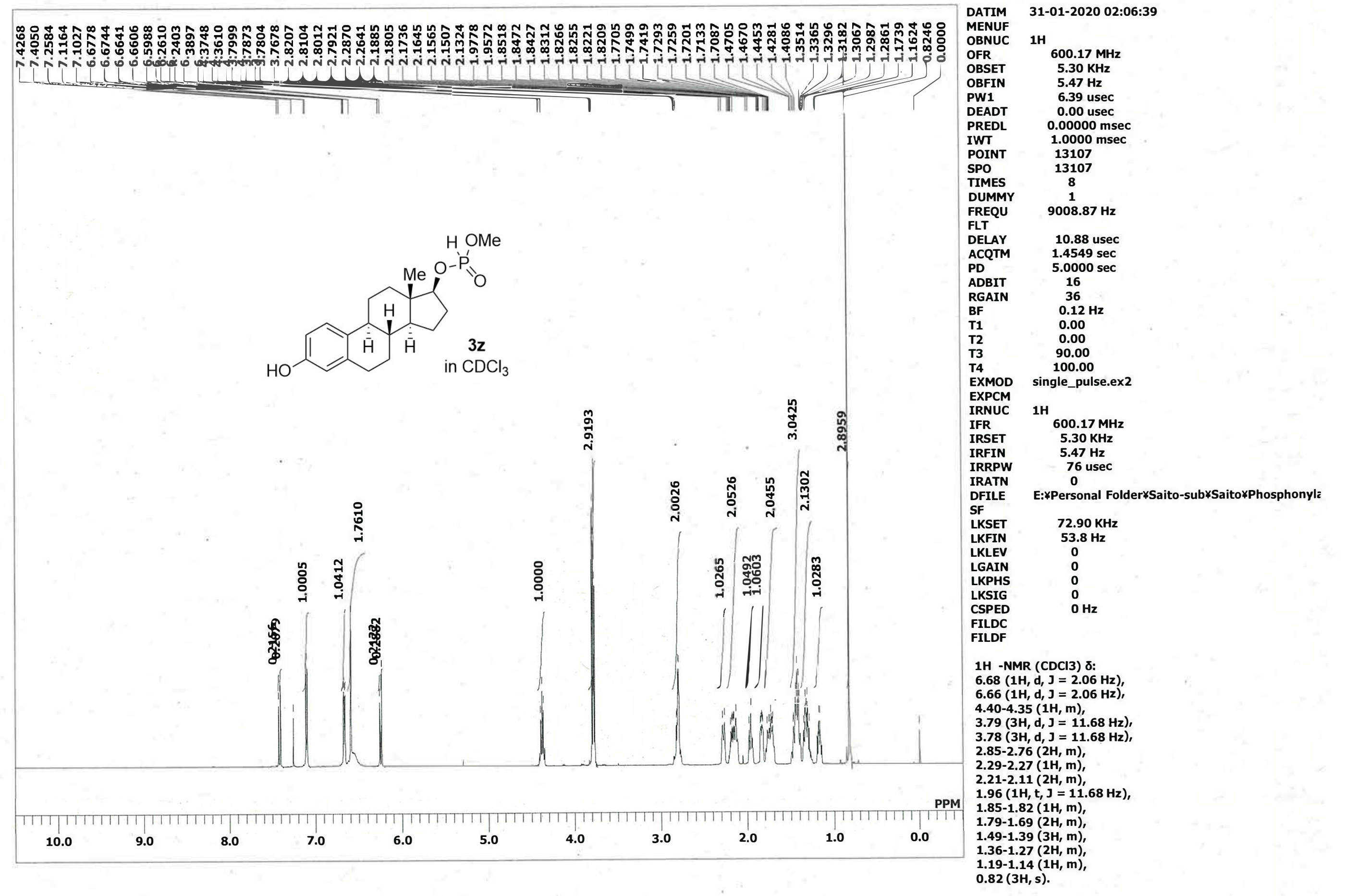


E:ҰPersonal Folder¥Saito-sub¥Saito¥Phosphonylation $¥$ estradiol-13C.als single pulse decoupled gated NOE
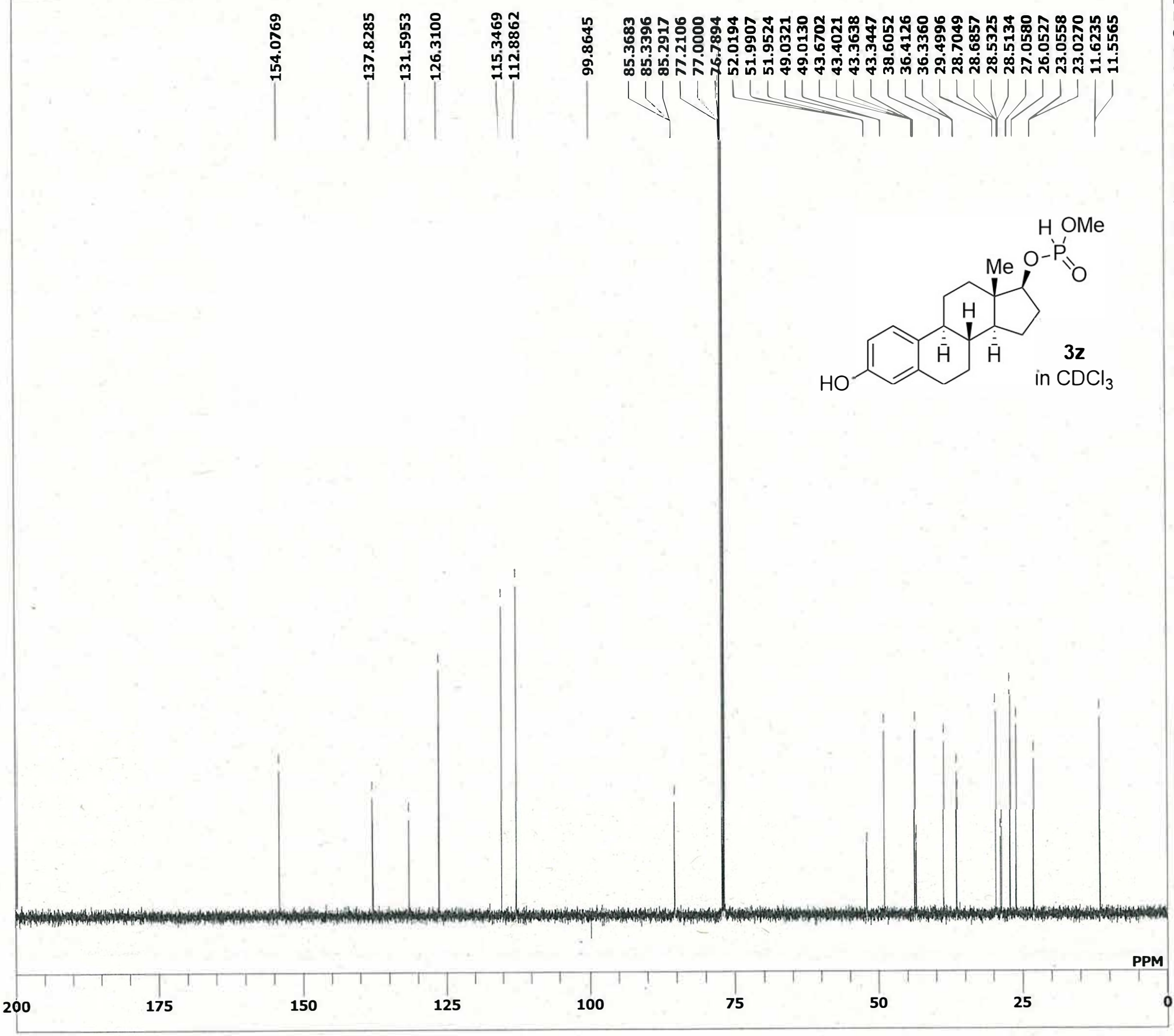

DATIM

31-01-2020 02:29:18 


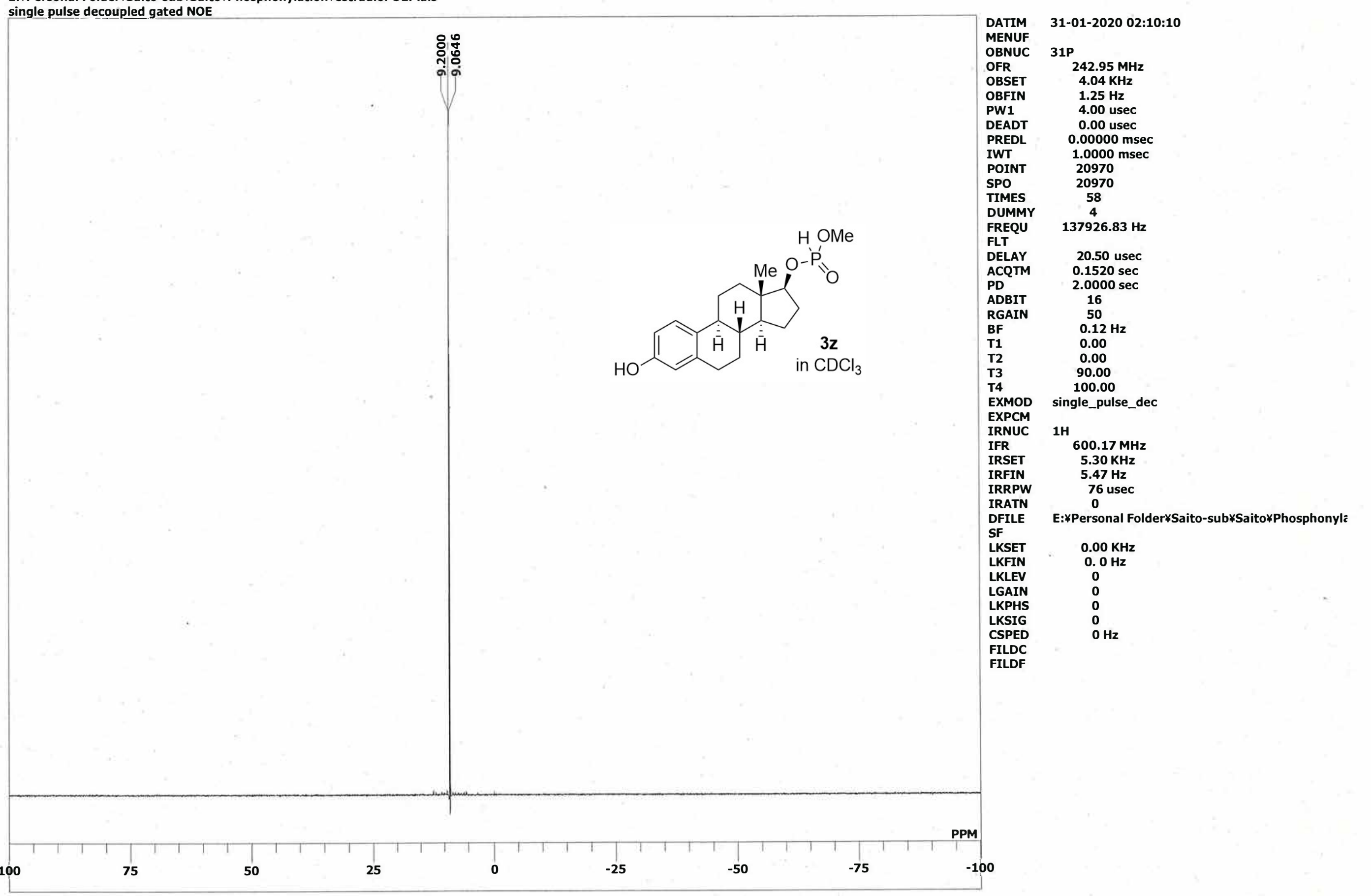




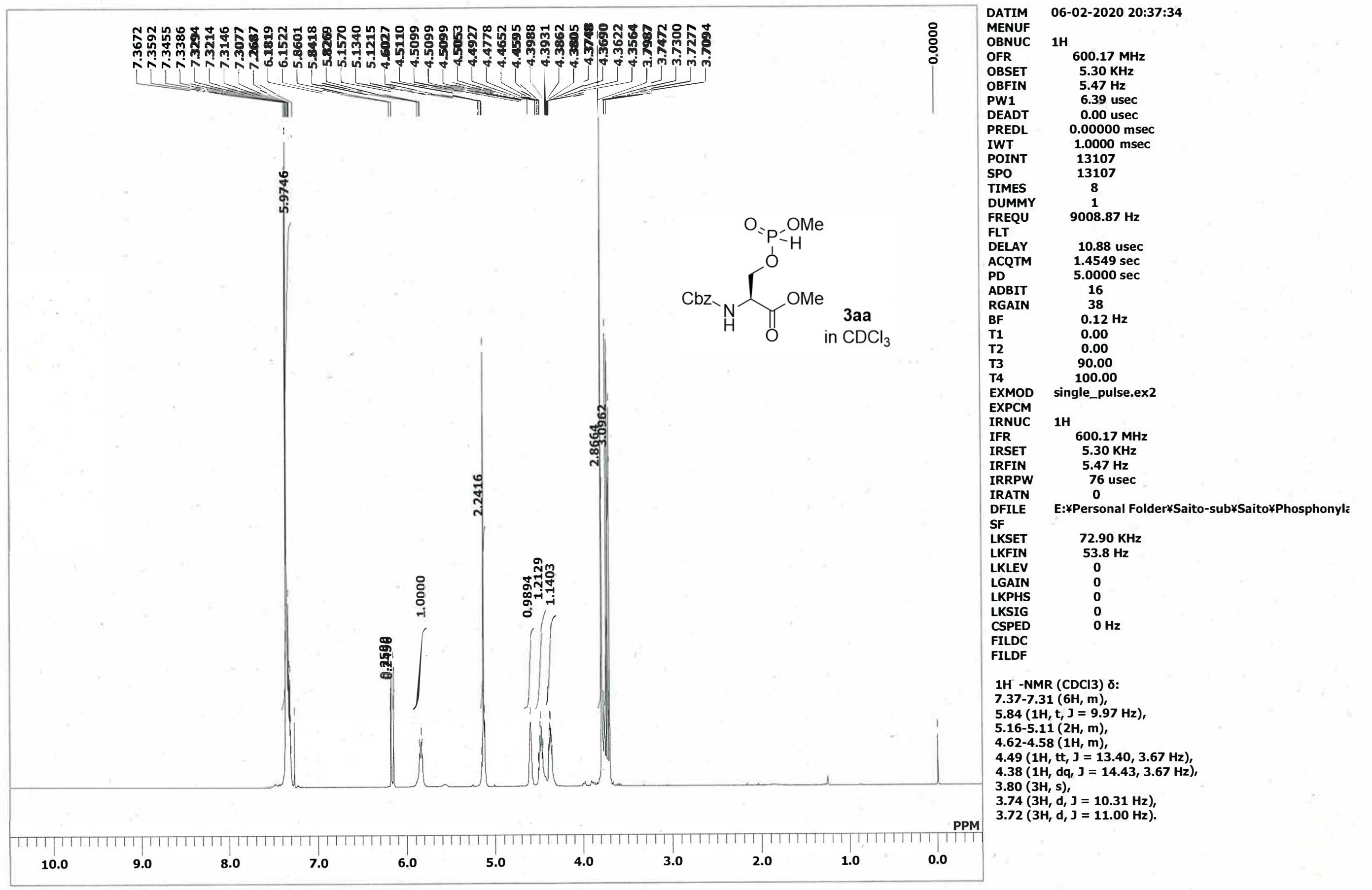


E:ҰPersonal FolderҰSaito-sub¥Saito¥Phosphonylation¥Z-Ser-OMe-13C.als single pulseidecoupled gated NOE
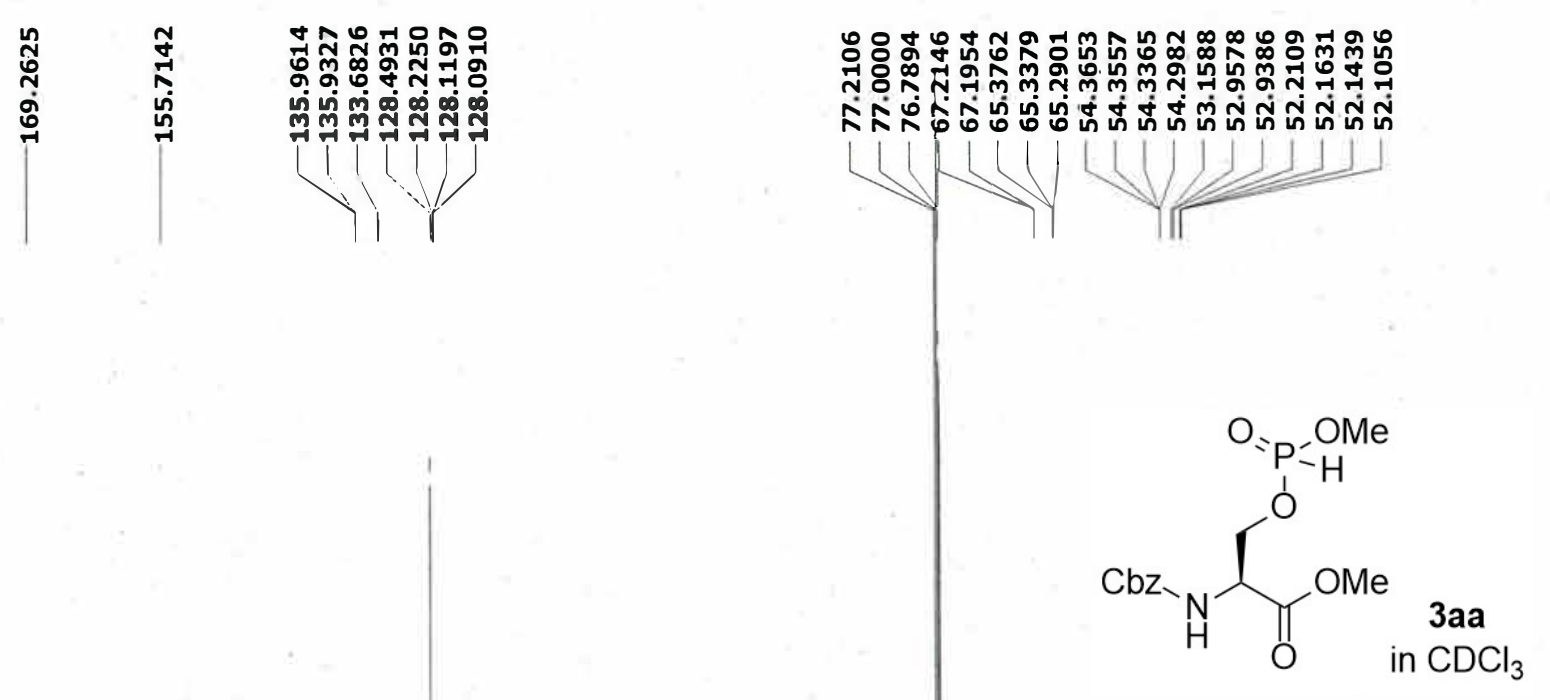

DATIM 06-02-2020 20:49:30

MENUF

OBNUC 13C

OFR $\quad 150.92 \mathrm{MHz}$

OBSET $\quad 8.52 \mathrm{KHz}$

PW1 $\quad 4.74 \mathrm{~Hz}$

DEADT $\quad 0.00$ usec

PREDL $\quad 0.00000 \mathrm{msec}$

IWT $1.0000 \mathrm{msec}$

\begin{tabular}{ll} 
POINT $\quad 26214$ \\
\hline
\end{tabular}

SPO $\quad 26214$

TIMES $\quad 122$

$\begin{array}{lc}\text { DUMMY } & 4 \\ \text { FREQU } & 37878.21 \mathrm{~Hz}\end{array}$

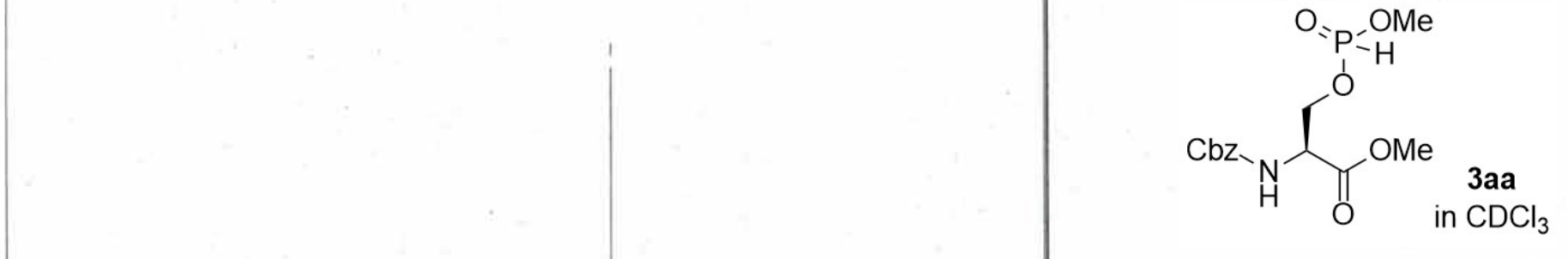

DELAY $\quad 21.12$ usec

PD $2.0000 \mathrm{sec}$

PDB

RGAIN

BF

T1

16

T2

T3 $\quad 90.00$

EXMOD single_pulse_dec

EXPCM

IRNUC

IFR

$1 \mathrm{H}$

IRFIN $\quad 5.47 \mathrm{~Hz}$

IRRPW $\quad 76$ usec

0

DFILE E:ҰPersonal Folder¥Saito-sub¥Saito¥Phosphonyla

$72.90 \mathrm{KHz}$

LKFIN $\quad 53.8 \mathrm{~Hz}$

0

LGAIN

KPHS

KSIG

FILDC

FILDF

0

$\mathbf{0}$
$\mathbf{0}$

$\mathbf{O H z}$ 
E:ҰPersonal Folder*Saito-sub¥Saito¥Phosphonylation¥Z-Ser-OMe-31P.als

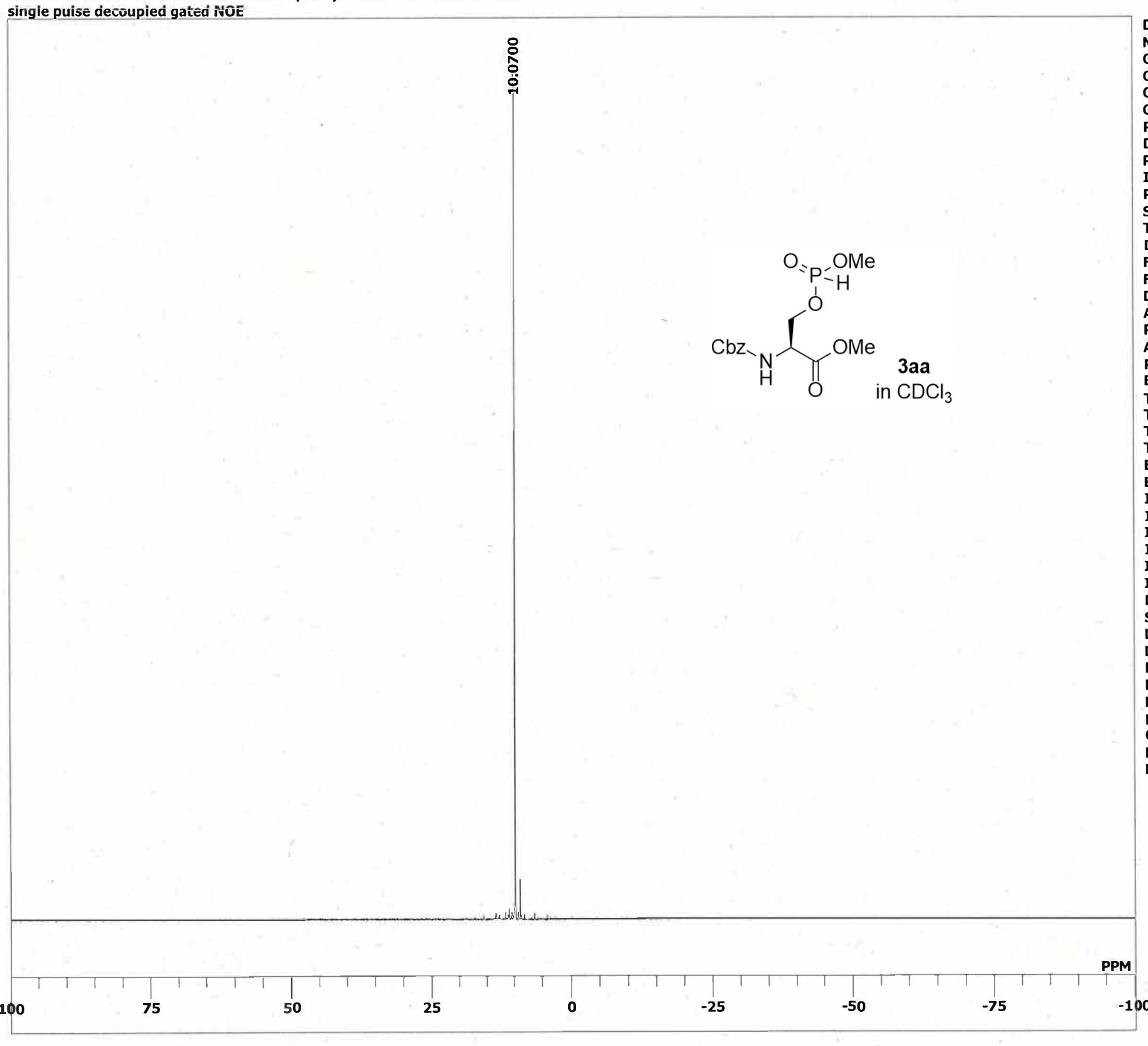

DATIM

$31 \mathrm{P}$

OBSET

DEADT

TIMES

TIM

FREQU

DELAY

CQTM

ADBIT

T1

T2

T3

EXMOD

EXPCM

IRNUC

IRNUC
IFR
IRSET

IRSET

IRFIN

IRRPW

SF

SF

LFIN

LKFIN

LKLEV

LKPHS

LKIG

LSPED

FILDC

FILDC

$242.95 \mathrm{MH}$

$4.04 \mathrm{KHz}$

$4.00 \mathrm{Hsec}$

0.00 usec

0.00 usec

$0.00000 \mathrm{msec}$

$1.0000 \mathrm{mse}$

20970

102

$137926.83 \mathrm{~Hz}$

20.50 usec

$0.1520 \mathrm{sec}$

$2.0000 \mathrm{sec}$

16

50
$0.12 ~ H z$

0.00

0.00

100.00

single_pulse_dec

$1 \mathrm{H}$

$600.17 \mathrm{MHz}$

$5.30 \mathrm{KHz}$

$5.47 \mathrm{~Hz}$

76 us

E:ҰPersonal Folder¥Saito-sub¥Saito¥Phosphonyla

$0.00 \mathrm{KHz}$

0
0

0 


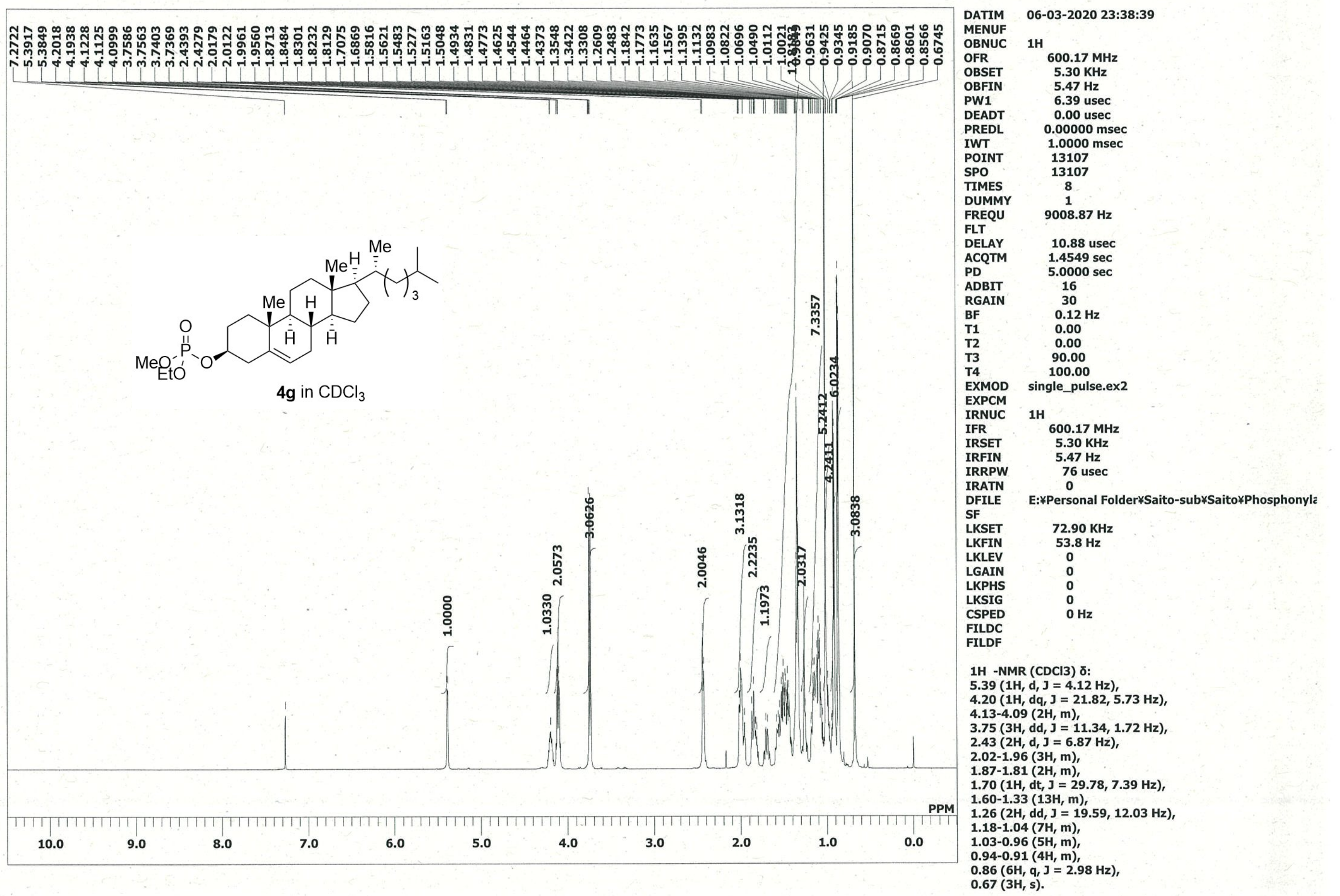




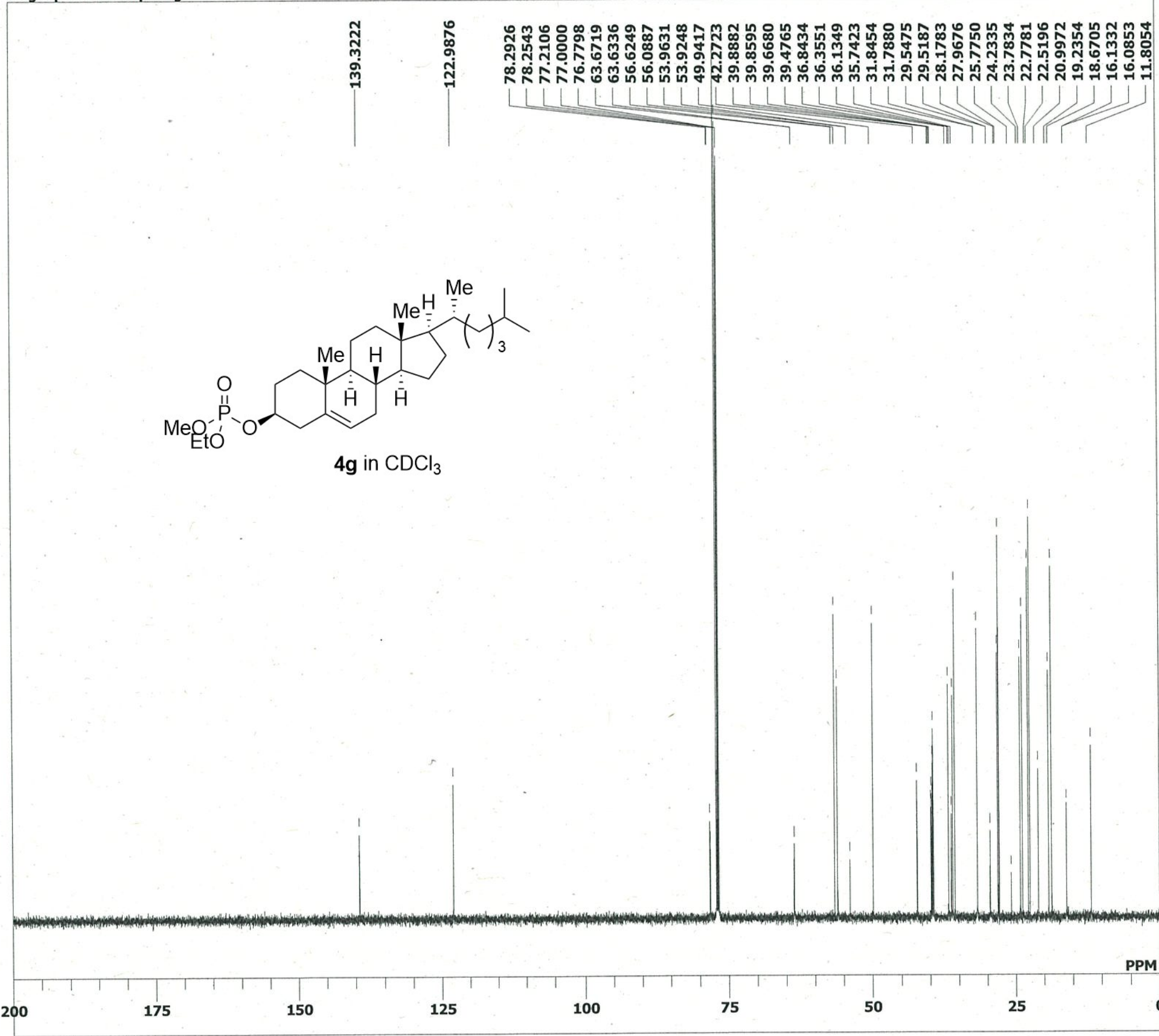


E:*Personal Folder¥Saito-sub¥Saito*Phosphonylation*cholesterol-ox31P.als

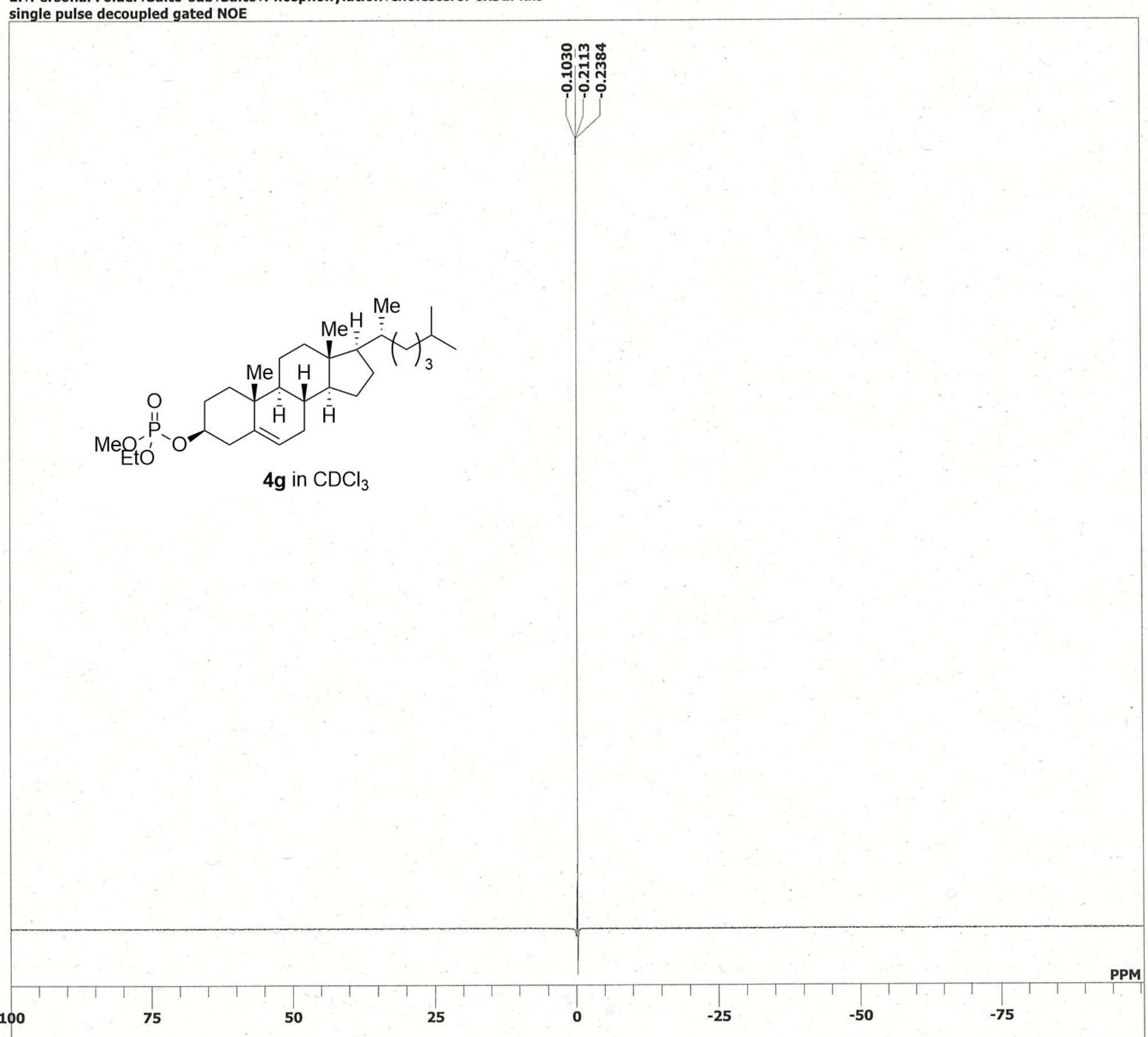

06-03-2020 23:44:44

OFR $\quad 242.95 \mathrm{MHz}$

OBSET $\quad 4.04 \mathrm{KHz}$

OBFIN $\quad 1.25 \mathrm{~Hz}$

PW1 4.00 usec

DEADT $\quad 0.00$ usec

PREDL $\quad 0.00000 \mathrm{msec}$

IWT $\quad 1.0000 \mathrm{msec}$

26214

TIMES $\quad 26214$

122

FREQU $\quad 172411.16 \mathrm{~Hz}$

20.50 usec

ACQTM $\quad 0.1520 \mathrm{sec}$

ADBIT $\quad 16$

$\begin{array}{ll}\text { RGAIN } & 50 \\ \text { BF } & 0.12 \mathrm{~Hz}\end{array}$

T2

T3

0.00
90.00
100.00

single_pulse_dec

EXPCM

RN

IFR

1H

IRFIN

IRRPW

DFILE

SF

LKSET

LKFIN

KLEV

LGAIN

LKPHS

LSIG

CSPED

FILDC

FILDF 